\title{
MUCH ADO ABOUT MICROBUNCHING: COHERENT BUNCHING IN HIGH BRIGHTNESS \\ ELECTRON BEAMS
}

\author{
A DISSERTATION \\ SUBMITTED TO THE DEPARTMENT OF APPLIED \\ PHYSICS AND THE COMMITTEE ON GRADUATE \\ STUDIES \\ OF STANFORD UNIVERSITY \\ IN PARTIAL FULFILLMENT OF THE REQUIREMENTS \\ FOR THE DEGREE OF \\ DOCTOR OF PHILOSOPHY
}

Daniel Ratner

May, 2011

Work supported in part by US Department of Energy contract DE-AC02-76SF00515.

SLAC National Accelerator Laboratory, Menlo Park, CA 94025 
Abstract The push to provide ever brighter coherent radiation sources has led to the creation of correspondingly bright electron beams. With billions of electrons packed into normalized emittances (phase space) below one micron, collective effects may dominate both the preservation and use of such ultra-bright beams. An important class of collective effects is due to density modulations within the bunch, or microbunching. Microbunching may be deleterious, as in the case of the Microbunching Instability (MBI), or it may drive radiation sources of unprecedented intensity, as in the case of Free Electron Lasers (FELs). In this work we begin by describing models of microbunching due to inherent beam shot noise, which sparks both the MBI as well as SLAC's Linac Coherent Light Source, the world's first hard X-ray laser. We first use this model to propose a mechanism for reducing the inherent beam shot noise as well as for predicting MBI effects. We then describe experimental measurements of the resulting microbunching at LCLS, including optical radiation from the MBI, as well as the first gain length and harmonic measurements from a hard X-ray FEL. In the final chapters, we describe schemes that use external laser modulations to microbunch light sources of the future. In these sections we describe coherent light source schemes for both both linacs and storage rings. 
Acknowledgments I would like to start by thanking my advisor, Alex Chao, who made this work and indeed my entire $\mathrm{PhD}$ and development as a physicist possible. I am indebted to all members of the accelerator department, including Karl Bane, Yuantao Ding, Huiping Geng, Gennady Stupakov, and Dao Xiang, who all contributed to various parts of the work presented here. The LCLS research, in chapters 4 and 5, was made possible by the entire LCLS commissioning team, and I am particularly indebted to Paul Emma and John Galayda who gave me the opportunity to join the project. Finally, I would like to thank Zhirong Huang, who had a part in shaping every chapter of this thesis. 


\section{Contents}

1 Introduction $\quad 1$

1.1 Noise Suppression . . . . . . . . . . . . . . . . . . . . . . . . 2

1.2 Microbunching Instability . . . . . . . . . . . . . . . . . . . . 2

1.3 LCLS Gain Length and Harmonics Results . . . . . . . . . . . . . . . . . . . . . . 3

1.4 Compressed Harmonic Generation . . . . . . . . . . . . . . . . . . . . . . . . . . . . 3

1.5 Steady-State Microbunching . . . . . . . . . . . . . . . . . . . . . . 4

1.6 Longitudinal Space Charge . . . . . . . . . . . . . . . . . . . . . . . . . 4

1.7 Phase Shift Equivalence to Undulator Taper . . . . . . . . . . . . . . . . . . 5

2 Shot Noise Suppression for Electron Beams

2.1 Introduction . . . . . . . . . . . . . . . . . . . . . . 7

2.2 Analytical Model . . . . . . . . . . . . . . . . . . . . . . . 8

2.2.1 Noise Factor . . . . . . . . . . . . . . . . . . . . . 8

2.2.2 Expectation Value of Noise Factor . . . . . . . . . . . . . . . . . . 8

2.2.3 Analytical Expression: Weak Interaction . . . . . . . . . . . . . . 10

2.2.4 Numerical Approximation: Strong Interaction . . . . . . . . . . . . . . . . . . 11

2.3 Space Charge Case . . . . . . . . . . . . . . . . . . . . . . . . . . 12

2.3.1 Space Charge Interaction . . . . . . . . . . . . . . . . . . . . 12

2.3.2 Space Charge Fourier Transform . . . . . . . . . . . . . . . . . . . 14

2.3.3 Space Charge Simulation . . . . . . . . . . . . . . . . . . 14

2.3.4 Validity of 1D model . . . . . . . . . . . . . . . . . . . 14

2.4 Undulator Radiation Case . . . . . . . . . . . . . . . . . . . . . . . . . . . . . 15

2.4.1 Undulator Radiation Interaction . . . . . . . . . . . . . . . . . . . 15

2.4.2 Undulator Fourier Transform . . . . . . . . . . . . . . . . . . . . 16

2.4 .3 Undulator Simulation . . . . . . . . . . . . . . . . . . . . 17

2.4.4 Undulator Numerical Integration . . . . . . . . . . . . . . . . . . . . . . 18

2.5 Example Parameters . . . . . . . . . . . . . . . . . . . . . . 18

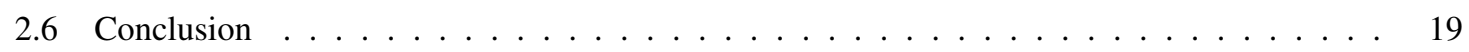

2.7 Acknowledgments . . . . . . . . . . . . . . . . . . . . . . . 19

2.8 Quadratic Term $\left(\Gamma_{2}\right)$ for Helical Undulator . . . . . . . . . . . . . . . . . . . . . . . 20

2.9 Bunching at the Average Inter-Particle Spacing . . . . . . . . . . . . . . . . . 21

2.10 Effects of Energy Spread and Modulation . . . . . . . . . . . . . . . . . . . . . . 24 
viii

2.10 .1 Energy Modulation from Noise Suppression . . . . . . . . . . . . . . . . . . 24

2.10 .2 Suppression Wavelength Limit . . . . . . . . . . . . . . . . . . . . 25

2.10 .3 Energy Spread for FEL . . . . . . . . . . . . . . . . . . . 25

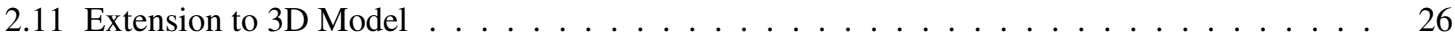

2.11 .1 Analytical Comparison of 1D and 3D models . . . . . . . . . . . . . 27

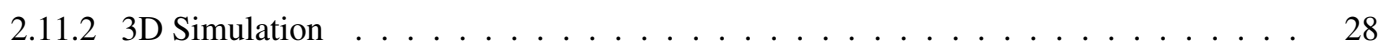

3 6D Shot Noise Model of Microbunching 31

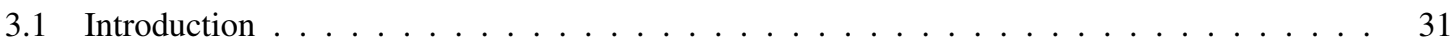

3.2 Model Assumptions . . . . . . . . . . . . . . . . . . . . . . . . . . . . 31

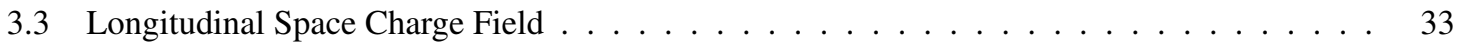

3.4 Expectation Value of Bunching Factor Squared $\ldots \ldots \ldots \ldots \ldots$

3.4.1 Weak Modulation Expansion . . . . . . . . . . . . . . . . . . . . 34

3.4 .2 Initial Distribution $\ldots \ldots \ldots \ldots$. . . . . . . . . . . . . . 36

3.4 .3 Weak LSC Limit $\ldots \ldots \ldots \ldots \ldots \ldots \ldots \ldots$

3.4 .4 Evaluating the Quadratic Term . . . . . . . . . . . . . . . . . 37

3.4.5 Evaluating Linear Term Expectation Value . . . . . . . . . . . . . . 40

3.5 Results . . . . . . . . . . . . . . . . . . . . . . . . . . . . . 41

3.5.1 Quadratic Term: Short Impedance Section . . . . . . . . . . . . . . . . . . . . 41

3.6 LCLS Results . . . . . . . . . . . . . . . . . . . . . . . . 42

3.6.1 Comparison of 6D and 2D Models . . . . . . . . . . . . . . . . 43

3.6.2 Comparison of Linear and Quadratic Terms . . . . . . . . . . . . . . . . . . . . . . 45

3.7 Transverse Motion Examples . . . . . . . . . . . . . . . . . . . . . . . . . . . . . 47

3.8 Discussion . . . . . . . . . . . . . . . . . . . . . . . . . . . . . . . . . 49

$4 \quad$ FEL Gain Length and Taper Measurements at LCLS

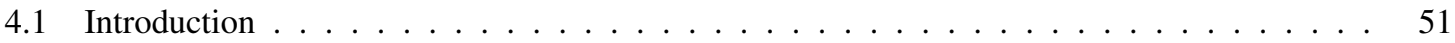

4.2 Gain Length Measurements . . . . . . . . . . . . . . . . . . . . 51

$4.2 .1 \quad$ Undulator Removal Method $\ldots \ldots \ldots \ldots$. . . . . . . . . . . . . 52

4.2 .2 Transverse Kick Method . . . . . . . . . . . . . . . . . . . . . . . . . . 52

4.2.3 Restarting FEL with Kick Method . . . . . . . . . . . . . . . . . . . . 52

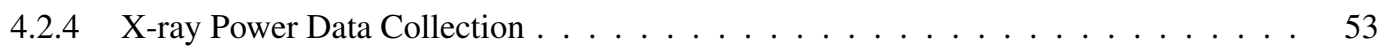

4.3 Results . . . . . . . . . . . . . . . . . . . . . . . . 54

$4.3 .1 \quad$ LCLS Gain Length . . . . . . . . . . . . . . . . . . . . . . 54

4.3.2 Gain Length vs. Energy Spread _ . . . . . . . . . . . . . . . . . . 55

4.3 .3 Saturation Taper $\ldots \ldots \ldots \ldots \ldots \ldots \ldots \ldots$

4.3 .4 Undulator Gaps (self-seeding) . . . . . . . . . . . . . . . . . 57

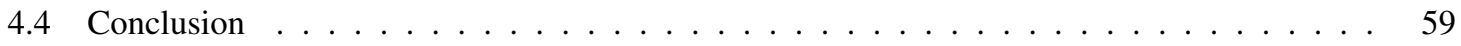

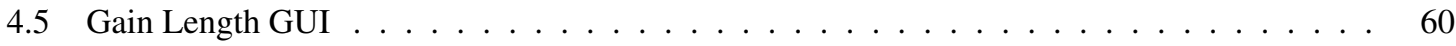


5 Second and Third Harmonic Measurements at the Linac Coherent Light Source 67

5.1 Introduction . . . . . . . . . . . . . . . . . . . . 67

5.2 Data Collection Methods . . . . . . . . . . . . . . . . . . . 68

5.2.1 Third Harmonic Method, Soft and Hard X-rays . . . . . . . . . . . . . . . . 68

5.2.2 Second Harmonic Method, Soft X-Rays . . . . . . . . . . . . . . . . . . 68

5.2.3 Intensity Measurement Methods . . . . . . . . . . . . . . . . . . . . . . 69

5.2.4 Transport Mirror Cutoff . . . . . . . . . . . . . . . . . . 69

5.3 Third Harmonic Measurements, Soft and Hard X-rays . . . . . . . . . . . . . . . . . . . 69

5.3 .1 Attenuator Scan . . . . . . . . . . . . . . . . . . 69

5.3 .2 K-edge Scan . . . . . . . . . . . . . . . . . . 71

5.4 Second Harmonic Measurements, Soft X-rays ～. . . . . . . . . . . . . . . . . . 73

5.5 Genesis Simulations . . . . . . . . . . . . . . . . . . . . . . . 74

5.6 Sensitivity to Beam Quality . . . . . . . . . . . . . . . . . . . . . 75

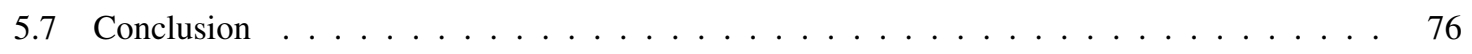

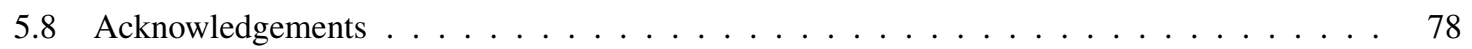

6 Two-Chicane Compressed Harmonic Generation of Soft X-Rays 79

6.1 Introduction . . . . . . . . . . . . . . . . . . . . . 79

6.2 Motivation . . . . . . . . . . . . . . . . . . . . . 80

6.2.1 Advantages of Compressed Seeding . . . . . . . . . . . . . . . . 80

6.2 .2 Advantages of Two Chicane CHG . . . . . . . . . . . . . . . . . 80

6.3 Analytical Model . . . . . . . . . . . . . . . . . . . . . . . 81

6.3 .1 Bunching Factor . . . . . . . . . . . . . . . . . 81

6.3.2 Error Self-Correction from Second Dispersive Region . . . . . . . . . . . . . . . 82

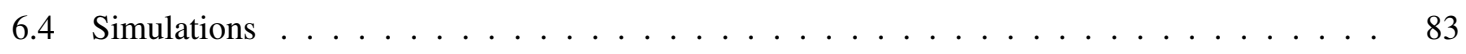

6.4 .1 Simulation Inputs . . . . . . . . . . . . . . . . . 83

6.4.2 Radiation ............................... 84

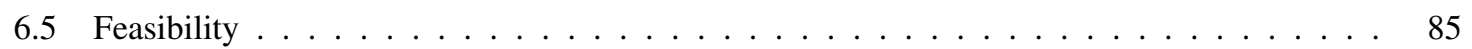

6.5 .1 ISR Induced Energy Spread . . . . . . . . . . . . . . . . . 85

6.5.2 Second Order Lattice Effects . . . . . . . . . . . . . . . . . . . . . . 85

6.5 .3 RF Phase and Wakefield Stability _. . . . . . . . . . . . . . 86

6.5 .4 Dispersion Leakage . . . . . . . . . . . . . . . . . . . . . . . 87

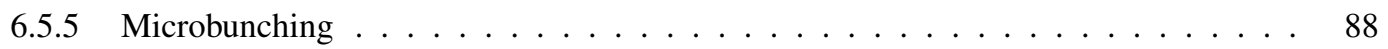

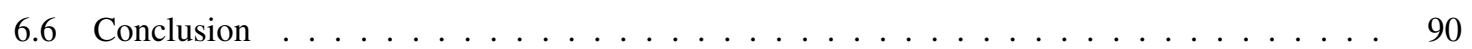

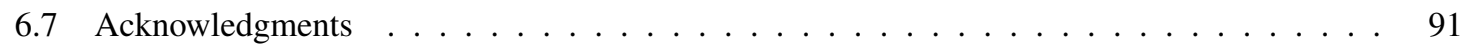

7 Steady-State Microbunching in a Storage Ring for Generating Coherent Radiation 93

7.1 Introduction . . . . . . . . . . . . . . . . . . . . . . 93

7.2 SSMB Mechanism . . . . . . . . . . . . . . . . . . . . . . . . . 94

7.2.1 Zero-Crossing SSMB . . . . . . . . . . . . . . . . . . 94

7.2 .2 Double Modulations . . . . . . . . . . . . . . . . . . . . 94

7.2.3 Analysis of Linearized Modulation . . . . . . . . . . . . . . . . . . 95 
7.3 Alternate Modes of SSMB _. . . . . . . . . . . . . . . . . . . . . 97

7.3.1 Modulation-Canceling Slippage Condition _. . . . . . . . . . . . . 97

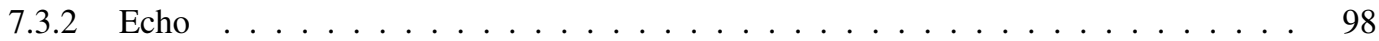

7.3.3 Long Wavelength Beating . . . . . . . . . . . . . . . . . . 101

7.3.4 Linearized RF bucket . . . . . . . . . . . . . . . . . . . . . 102

7.3.5 Hamiltonian View . . . . . . . . . . . . . . . . . . . . . 103

7.4 Example Radiation Sources from SSMB . . . . . . . . . . . . . . . . . . . . . . . . 104

7.4.1 EUV Source . . . . . . . . . . . . . . . . . . . . 104

$7.4 .2 \mathrm{THz}$ and Pump Probe Sources . . . . . . . . . . . . . . . . . . 107

7.5 Technical Challenges ........................... . . . . . . . . . . . . . . . . .

7.6 Conclusion . . . . . . . . . . . . . . . . . . . . . . . 108

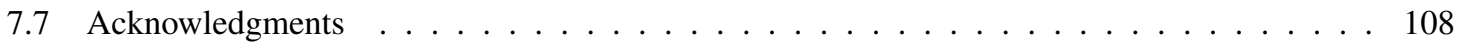

8 Numerical Calculation of Longitudinal Space-Charge in an Undulator 109

8.1 Introduction . . . . . . . . . . . . . . . . . . . . . . 109

8.2 Field Components . . . . . . . . . . . . . . . . . . . . . . . . . . . . . 109

8.2.1 Scalar Potential . . . . . . . . . . . . . . . . . . 110

8.2.2 Vector Potential . . . . . . . . . . . . . . . . . . . 110

8.2 .3 Bunch Shape and Integration . . . . . . . . . . . . . . . . . 111

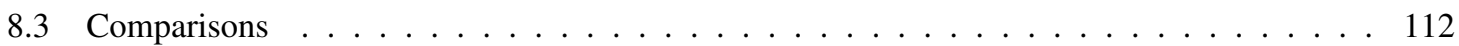

8.3.1 Straight Motion along Z Axis . . . . . . . . . . . . . . . 112

8.3.2 Straight Motion at an Angle . . . . . . . . . . . . . . . . . . 112

8.3.3 Coherent Synchrotron Radiation: Circular Motion _ . . . . . . . . . . . . . 113

8.3.4 Coherent Synchrotron Radiation: Undulator Motion . . . . . . . . . . . . . . . . 114

8.3.5 Space Charge Limit: Undulator Motion . . . . . . . . . . . . . . . . . 115

8.4 Extra Calculations . . . . . . . . . . . . . . . . . . . . 116

8.4.1 Integration Lengths . . . . . . . . . . . . . . . . . . . 116

8.4 CSR Radiation . . . . . . . . . . . . . . . . . . . . . 118

9 Enhancing FEL Power with Phase Shifters 121

9.1 Introduction . . . . . . . . . . . . . . . . . . . . . 121

9.2 One-Dimensional Analysis . . . . . . . . . . . . . . . . . . . . . . . . . 122

9.3 Equivalence to Undulator Taper . . . . . . . . . . . . . . . . . . . . . . 123

9.4 Numerical Results . . . . . . . . . . . . . . . . . . . . . . . . . . . . . . . . . . . 124

$9.4 .1 \quad 1-\mathrm{D}$ Simulations $\ldots \ldots \ldots \ldots \ldots \ldots$

9.4 .23 -D Simulations . . . . . . . . . . . . . . . . . 126

9.5 Conclusion . . . . . . . . . . . . . . . . . . . . . . 127

9.6 Acknowledgments . . . . . . . . . . . . . . . . . . . . . 127

A Comparison of 1D and 3D Models of Longitudinal Space Charge 131

A.1 Summary of Results . . . . . . . . . . . . . . . . . . . . . . . . . 131

A.2 Longitudinal Space Charge from Shot Noise . . . . . . . . . . . . . . . . . . . . . . 132

A.2.1 1D LSC Model . . . . . . . . . . . . . . . . . . . . 133 
A.2.2 3D LSC Model . . . . . . . . . . . . . . . . . . . . . . . . . . . . . . 134

A.3 Transverse Average . . . . . . . . . . . . . . . . . . . . . . . . . 138

A.3.1 Transverse Average: 1D Case . . . . . . . . . . . . . . . . . . . . . . . 138

A.3.2 Transverse Average: 3D Case, Numerical Approach _ . . . . . . . . . . . . . . 138

A.3.3 Transverse Average: 3D Case, Analytical Approach . . . . . . . . . . . . . . . . . . 140 


\section{List of Tables}

5.1 Nominal (middle column) and measured (right column) attenuation lengths for the fundamental and third harmonic at three different photon energies. The nominal and fitted attenuation lengths match well for the fundamental, but in general the fitted third harmonic values are lower than expected. Fitting for the the third harmonic attenuation length at hard $\mathrm{x}$-rays is not effective due to the low absorption of the third harmonic. . . . . . . . . . . . . .

5.2 Nominal (middle column) and measured (right column) second harmonic attenuation lengths for the gas detector. The nominal and fitted attenuation lengths match well for the fundamental, but in general the fitted second harmonic values are lower than expected. . . . . . . . . .

5.3 Genesis simulation parameters given for soft and hard $\mathrm{x}$-ray simulations. The simulations are consistent with measurements for both soft and hard x-rays. . . . . . . . . . . .

5.4 Summary of harmonic results. The second harmonic content in normal operation at soft xray wavelengths is below $0.1 \%$. The third harmonic content is as high as about $2 \%$ at normal operation, but can drop by an order of magnitude or more due to poor beam quality.

6.1 Parameter list for both 1D (MATLAB) and 3D (elegant) simulations. We choose L-band RF frequencies because the larger diameter structures will mitigate the effect of wakefield fluctuations. We have not listed bunch charge because without wakefield effects the simulations are charge-independent. . . . . . . . . . . . . . . . . . . . . . . . . . . 84

7.1 Example Parameters . . . . . . . . . . . . . . . . . . . . . . . . 106

8.1 Parameters used for undulator space-charge simulation. All lengths normalized to units of

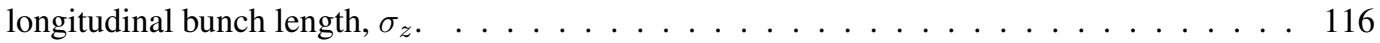

9.1 SASE Simulation Parameters . . . . . . . . . . . . . . . . . . . . . . . . . . . . . 124 


\section{List of Figures}

2.1 Schematic of our model system. Starting with an initial electron distribution function, $\Psi_{0}(z, \eta)$, the interaction and dispersive regions produce a final distribution function, $\Psi_{f}(\hat{z}, \hat{\eta})$. The dispersion may be positive or negative. . . . . . . . . . . . .

2.2 Schematic of an interaction near a density spike (solid green line). At left, for the space charge case, particles in the front half of the spike gain energy, while particles in the back half lose energy, and in positive dispersion, the density spike shrinks (dotted green line). We have a similar result for an interaction due to undulator radiation (right). At high frequency (spike much shorter than undulator resonant wavelength), all particles lose energy, but following a dispersive region with negative $R_{56}$ we still find a reduction in the density spike (dotted green

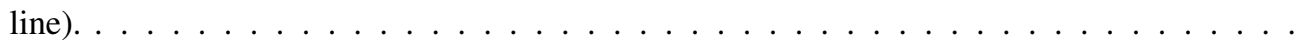

2.3 Space charge from a source sheet produces a change in energy $\left(h_{s c}\right)$ in a test sheet located at a distance $\zeta$. We calculate the energy modulation to the test sheet by averaging over the entire sheet (solid blue curve). Though $E_{z}$ (and thus $h_{s c}$ ) is not constant everywhere in the test sheet, we note that there is relatively little variation near the center of the sheet, as can be seen from $h_{s c}$ evaluated at radius $r=0$ (dashed green line) and $r=a / 2$ (dotted red line). .

2.4 On the left we show a histogram of particle density for particles loaded with random longitudinal positions. At right, following the interaction and dispersive regions, we find a reduction in noise in the equivalent histogram. (Example has $n_{0} a=5 \times 10^{2}, \Upsilon=1$ ). . . . . . .

2.5 Longitudinal distribution of particles in simulation before $(\times)$ and after $(*)$ the noise suppression process. For $\sigma_{\eta} \ll 1 / R_{56} n_{0}$ and a $1 \mathrm{D}$ beam, it is possible to show that the initially uncorrelated distribution gives way to a regularly spaced beam with inter-particle spacing $1 / n_{0}$ (see section 2.9). The regular structure amplifies bunching at very high frequencies, $k=2 \pi n_{0}$ and its harmonics, while suppressing $F(k)$ at frequencies below $2 \pi n_{0} \ldots \ldots \ldots$

2.6 A comparison of simulation and analytical results shows noise suppression as a function of frequency. With $\Upsilon \approx 1$ at high frequency, we find strong suppression. At low frequencies ( $k \lesssim 2 \pi / a$ ), we no longer have $\tilde{h}_{u}(k) \propto 1 / k$, so suppression is weaker for the given parameters. 17

2.7 A comparison of simulation, analytical result and numerical integral shows noise suppression at high frequency for $\Upsilon=1$. At low frequencies ( $m \sim 1$ ), we find $\tilde{h}_{u}(k)$ is approximately real (Eq. 2.31), and bunching increases to $F(k) \approx 1-2 n_{0} k_{0} R_{56} \operatorname{Im}\left[\tilde{h}_{u}\left(k_{0}\right)\right]+$

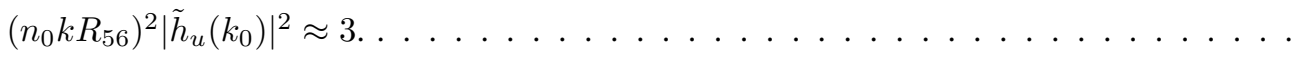

2.8 A close-up of Fig. 2.7 shows agreement with the analytical expression starts to fail for $m \gtrsim 2$, but the numerical integral matches well everywhere. . . . . . . . . . . . . 
2.9 A simulation example for 10 undulator periods shows good agreement with the numerical

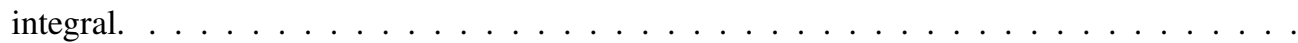

2.10 Simulations show the noise factor for both $N_{u}=1$ and $N_{u}=10$ as a function of $\Upsilon$. When the approximation $k^{2} N_{u} \lambda_{0} / n_{0} \gg 1$ is valid, the noise scales as $(1-\Upsilon)^{2}$. We have chosen $m$ so that $\tilde{h}_{u}(k)$ is approximately imaginary. . . . . . . . . . . . . . . . . . . .

2.11 The relative modulation between neighboring particles, $E_{k+1}-E_{k}$, is proportional to the initial distance between the particles (sheets), $\Delta z$. Simulation is for the undulator interaction, with $\Upsilon=1, n_{0} \lambda_{0}=10^{3}$. Particles that are closer (farther) than the inter-particle spacing, $\Delta z<1 / n_{0}$, lose (gain) energy relative to the previous particle, and move away (closer) in negative dispersion. . . . . . . . . . . . . . . . . . . . .

2.12 Phase space of the particles following both the interaction and dispersive regions. Example is for the undulator case with uniform initial energy, $\sigma_{\eta}=0 . \ldots \ldots$

2.13 RMS energy modulation, $\langle\Delta \eta\rangle$, induced by the interaction (Eq. 2.50) is compared to the result of repeated simulations for the undulator case. The final energy spread is given as a function of the particle per wavelength, $n_{0} \lambda_{0}$, for $N_{u}=1$ and 10, and initial energy spread $\sigma_{\eta}=0 \ldots \ldots \ldots \ldots \ldots \ldots \ldots \ldots \ldots \ldots \ldots \ldots \ldots \ldots \ldots \ldots \ldots \ldots \ldots \ldots \ldots \ldots \ldots$

2.14 Noise suppression for the case $N_{u}=1$ with $\langle\Delta \eta\rangle \approx \sigma_{\eta} / 5$. For the parameters given, $\lambda_{\min } \approx \lambda_{0} / 8$. With higher charge density, $n_{0}$, suppression extends to shorter wavelengths. .

2.15 Comparison of 1D and 3D models for both $r=0$ (red curves) and transverse average (blue). When evaluated at $r=0$, the 1D and 3D models diverge at high frequencies $\left(\xi_{b} \gg 1\right)$, as shown by Venturini. However, when averaged transversely, the $1 \mathrm{D}$ and 3D results are nearly identical even at high frequency. The numerical integral, which matches Venturini's Eq. 12 and reproduces the $r_{1}=r_{2}=0$ case, also confirms our analytical result. These results are for a transverse flat-top distribution. See Fig. 3.6 for the equivalent results for a Gaussian transverse distribution.

2.16 Noise suppression for the 3D space charge case. The suppression starts to fail when the number of particles is too low, so our simulations improve with larger particle number. Ideally we would simulate higher particle densities, but are limited by computational times. . . . . .

3.1 Schematic of the interaction-dispersion model system. Longitudinal space charge causes an energy modulation, which then produces a density modulation following a dispersive region. The dispersion may be positive or negative. . . . . . . . . . . . . . . . . .

3.2 Schematic of the LCLS beamline through the first bunch compressor at the time of measurements (figure from Ref. [1]). The current beamline is nearly identical except for the addition of a laser heater in the dogleg following LOb. . . . . . . . . . . . . . . . .

3.3 The integrated OTR signal as a function of the QB quadrupole strength at $250 \mathrm{pC}$ bunch charge (courtesy D. Dowell et al.) . . . . . . . . . . . . . . . . . . .

3.4 Twiss parameters for the LCLS beamline showing the small waist in front of the dogleg. Experimental attempts to decrease or increase the waist were not successful, likely because second order effects resulting from changes to the large beta function in the dogleg dominate.

3.5 OTR intensity gain for $250 \mathrm{pC}$ charge at OTR12 as a function of the optical wavelength. . . 
3.6 Total gain for $6 \mathrm{D}$ and $2 \mathrm{D}$ models (or 3D and $1 \mathrm{D}$ if we do not count angular components) is roughly equivalent. In the high frequency limit, the gains differ by a factor of $4 / 3$, due to our choice of gaussian transverse distribution. For flat-top distributions, the difference is even smaller (Fig. 2.15) ～. . . . . . . . . . . . . . . . . . . .

3.7 Suppression of shot noise from linear term. By setting $R_{56}$ so that the linear term has a negative contribution to the noise, we can suppress the bunching factor below the shot noise level. . . . . . . . . . . . . . . . . . . . . . . . . .

4.1 A transverse dipole kick suppresses the FEL process. The electrons continue to travel through the undulator line, but the kick smears the bunching and misaligns the radiation and electron

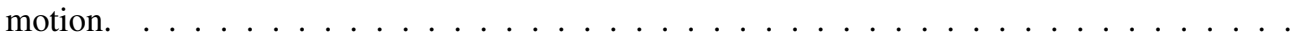

4.2 Gain lengths measured with both the undulator removal and dipole corrector kick methods. Distances are for total undulator length and do not include the breaks between segments. . .

4.3 At left, the primary FEL spot at saturation is centered near $x=0.25 \mathrm{~mm}$. At right, after strongly suppressing the main FEL peak (faintly visible around $x \approx 0.5 \mathrm{~mm}$ ), secondary FEL spots emerge on either side. (Though stronger than the main FEL peak, these spots are still several orders of magnitude weaker than the saturated FEL). The left-hand secondary spot is aligned with the straight portion of the orbit distortion. . . . . . . . . . .

4.4 Cartoon showing the difficulty of suppressing the soft X-ray FEL. The kick temporarily stops the FEL process, but the bunching can either realign (lower spot) or can restart from shot noise during the straight portion of the distorted orbit (upper spot), creating secondary FEL radiation. Additional kicks can suppress this secondary radiation, or the camera region of interest can select only the primary central spot. In this cartoon, down corresponds to positive $\mathrm{X}$ on the YAG screen $\ldots \ldots \ldots \ldots \ldots \ldots \ldots \ldots$

4.5 Sample gain length taken with the GUI showing gain length of $2.85 \pm 0.06 \mathrm{~m}$ at $13.6 \mathrm{GeV}$ using the dipole corrector method. The points within the dotted black lines are used to calculate the gain length (shown as solid green line). The high power levels of the first two data points are due to a secondary FEL spot as seen in Fig. 4.3. Colors of data points correspond to the state of the camera neutral density filters while taking the data. Green boxes represent inserted undulators. . . . . . . . . . . . . . . . . . . . .

4.6 A gain length measurement of $3.3 \mathrm{~m}$ agrees well with Genesis simulations for a beam with

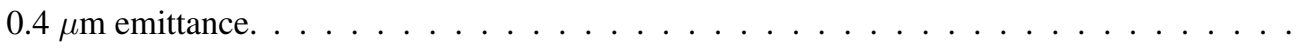

4.7 Genesis simulation showing power as a function of longitudinal bunch position (left). The peak power occurs in the current spikes (right). For this simulation, the spikes had emittances

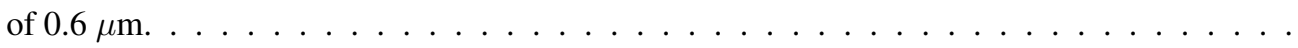

4.8 Gain length of $1.62 \pm 0.15 \mathrm{~m}$ taken at $4.7 \mathrm{GeV}$ using the undulator pull method and the YAGXRAY screen. Only four points are used due to the time required to pull each undulator and the danger of damaging YAGXRAY with high FEL power. . . . . . . . . . .

4.9 Gain length as a function of energy spread following the laser heater, with Ming Xie scaling shown for $0.4,0.5 \mu \mathrm{m}$ emittances. We note the increased gain length with no laser heating $(0$ $\mathrm{keV})$, hinting at the importance of the laser heater to suppress the microbunching instability. The nominal heater value induces a $20 \mathrm{keV}$ energy spread. . . . . . . . . . . . . . . . 
4.10 Experimentally-optimized undulator taper yielding an FEL-induced average electron energy loss of nearly $9 \mathrm{MeV}$ with the electron beam energy at $13.5 \mathrm{GeV}$. (Measurement taken on

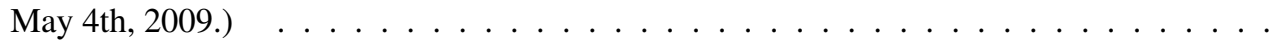

4.11 Post-saturation FEL pulse energy for a taper with nearly $9 \mathrm{MeV}$ final average electron energy loss. Genesis simulations for a $0.4 \mu \mathrm{m}$ emittance beam agree well, but have slightly lower

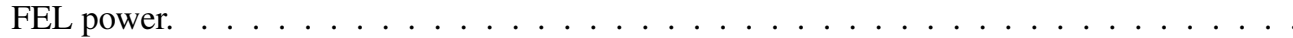

4.12 At left, measured FEL-induced electron energy loss as a function of dipole kick at undulator 11. The addition of a saturation taper can increase the FEL output by a factor of greater than 2. At right, simulation results show a post-saturation taper increasing the FEL output by greater than a factor of $3 \ldots \ldots \ldots \ldots \ldots \ldots \ldots$

4.13 As expected, removing an undulator section suppresses the FEL growth. Without a phase error (and ignoring diffraction effects), we would expect the gap to delay the gain length curve by one section. However, we can see that removing one undulator (either 10 or 11), delays the gain curve by approximately two sections, suggesting that an additional section is required to restore the correct phase relation between electrons and X-rays. Removing two sections $(10$ and 11$)$, has a much more deleterious effect. . . . . . . . . . . . . . .

4.14 Scan of undulator $11 \mathrm{~K}$ parameter when all undulators are inserted (left) and with undulator 12 removed (right). We see that the optimal K parameter for undulator 11 is near the nominal value $(0 \mathrm{~mm}$ offset $)$ when all of the undulators are inserted. However, the optimal $\mathrm{K}$ parameter shifts to smaller values when undulator 11 is followed by a gap. . . . . . . . . . . . .

4.15 Detuning the undulators before and/or after the missing undulator partially recovers the lost FEL power. We note that a perfect phase match can at best result in a one undulator section

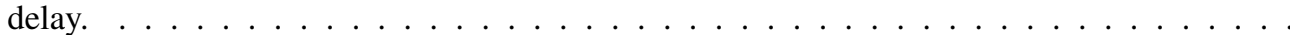

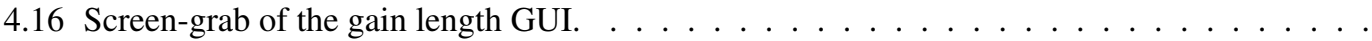

5.1 Schematic of the harmonic measurements. Attenuators block the fundamental and second harmonic, allowing measurement of the third harmonic on the Direct Imager YAG screen. Alternatively, we can isolate the second harmonic by measuring the intensity at the P3S1 YAG screen; the soft x-ray mirrors, upstream of P3S1, absorb the third harmonic. We again rely on the attenuators to block the fundamental. . . . . . . . . . . . . . . .

5.2 Schematic of the mirror cutoff measurement. For the soft x-ray line, the ratio of intensities measured on YAG screens P3S1 and P2S gives the transmission for a single mirror, and the total transmission is assumed to be the cube of this ratio. Alternatively, we can compare the intensity on P3S1 (downstream of all mirrors) directly against the intensity at the gas detector (upstream of all mirrors). For the hard x-ray line, attenuators block the fundamental, and we calculate the square of the ratio of third harmonic measured before $(\mathrm{P} 3 \mathrm{H})$ and after $(\mathrm{P} 2 \mathrm{H})$ the second mirror. . . . . . . . . . . . . . . . . . . . . . . . . 
5.3 Transmission plots show the photon energy cutoffs of the soft x-ray (left plot) and hard x-ray (right plot) experimental beamlines. On the left, blue circles show the cube of the ratio of intensities on P3S1 and P2S, while the red diamonds give the ratio from P3S1 to the full pulse energy. Both results match the expected transmission (dashed green curve) [2, 3]. On the right, blue circles give the square of the ratio of intensities measured on $\mathrm{P} 3 \mathrm{H}$ and $\mathrm{P} 2 \mathrm{H}$. The dashed curve gives the expected transmission at the nominal 0.0773 degree angle; however, we find a better match for 0.083 degree angle (dot-dash curve). Hard x-ray measurements rely on the third harmonic, resulting in lower resolution than for the soft x-ray measurements. In both cases, the experimental curves are normalized to one at the maximal value. . . . . .

5.4 Examples of third harmonic measurements for fundamental FEL photon energies of $0.9 \mathrm{keV}$ (left), $6 \mathrm{keV}$ (center) and $8 \mathrm{keV}$ (right). Blue circles show the experimental data, red dotted lines show linear one-parameter fits using the nominal attenuation lengths, while solid green lines show three-parameter fits to both the harmonic content and attenuation lengths. The black dot-dash line shows the expected drop in intensity for the fundamental only. We typically find around $2 \%$ third harmonic for soft x-rays, with between $0.2-2 \%$ third harmonic at hard x-rays. The $4 \%$ level found from the one-parameter fit at $8 \mathrm{keV}$ (right plot) is higher than expected, and may be artificially high due to a lack of measurement points dominated by the fundamental. . . . . . . . . . . . . . . . . . . . .

5.5 Transmission for the zirconium filter as a function of photon energy. The filter is made of 100 $\mu \mathrm{m}$ of zirconium (dashed blue) and $1 \mathrm{~mm}$ silicon (dotted green), with the combined transmission given in solid red. The zirconium K-edge manifests as a sharp drop in transmission at $18 \mathrm{keV} \ldots \ldots \ldots \ldots \ldots \ldots \ldots \ldots \ldots$

5.6 Plot of measured intensity on the Direct Imager YAG screen (Y-axis) vs. photon energy (Xaxis), with the FEL tuned to near $6 \mathrm{keV}$ fundamental and the zirconium attenuator inserted. The drop-off in intensity as the fundamental photon energy crosses $6 \mathrm{keV}$ corresponds to the zirconium K-edge at $18 \mathrm{keV}$; we conclude that the drop in intensity is due to third harmonic content. With the zirconium filter blocking the fundamental, we find that the remaining radiation is almost entirely third harmonic. Electron energy jitter and FEL bandwidth broaden the otherwise sharp K-edge seen in Fig. 5.5 . . . . . . . . . . . . . . . . . . .

5.7 An example image in the soft x-ray beam line (P3S1) shows the characteristic double lobe structure of the second harmonic. Though gas and solid (beryllium) attenuators strongly suppress the fundamental in this image, a small amount of fundamental radiation remains (gaussian mode background). Diffraction from the beryllium attenuators produces the uneven

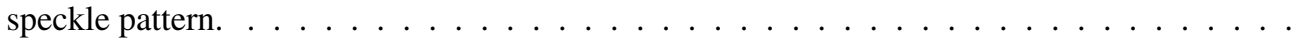

5.8 We measure the harmonic component by varying the gas attenuator strength for $0.9 \mathrm{keV}$ (left) and $1 \mathrm{keV}$ (right) fundamental photon energies. Blue circles show experimental data, red dotted lines show one-parameter fits using the nominal attenuation lengths, and solid green lines show three-parameter fits to both the harmonic content and attenuation lengths. The black dot-dash line shows the expected attenuation of the fundamental only. We find approximately $0.05-0.1 \%$ second harmonic content. . . . . . . . . . . . . . 
5.9 Energy spread measured as a function of laser heater power. To determine energy spread, we measure the beam width in the dispersive region a few meters downstream from the laser heater. The energy spread in $\mathrm{keV}$ is approximately $\Delta E=8 \sqrt{P_{L}}$, with the laser heater power, $P_{L}$, given in $\mu \mathrm{J}$ [4]. Note that at low laser power trickle heating causes a noticeable bump in the energy spread. . . . . . . . . . . . . . . . . . .

5.10 Percentage of harmonic power vs. laser heater setting. As the FEL performance drops (dotted red line), the percentage of third harmonic also drops (solid green), indicating enhanced sensitivity to beam quality at higher harmonics. The FEL performance was determined by energy loss scans, which measure the energy loss of the electrons due to the FEL process [5]. We note that even at the nominal heating level, the harmonic content only reached $0.2 \%$, likely due to the poor FEL performance on the day of the measurement. . . . . . . . . . .

6.1 Diagram of CHG scheme. a) The first accelerator section raises the particle energy to $E_{a}$ and introduces a linear chirp, $h$. After modulating with a laser, the first dispersive section, BC1, compresses the beam and over-bunches the modulation. A second RF section accelerates the particles to $E_{b}$, and cancels the chirp of the first section. The final dispersive section, BC2, unwinds the over-bunching. b) Operating $\mathrm{BC} 1$ in over-compression rotates the electron beam head-to-tail, allowing the use of a chicane for both $\mathrm{BC} 1$ and $\mathrm{BC} 2$. In both cases it is possible to add a third accelerator section to reach a final energy of $E_{f} \ldots \ldots \ldots$. . . . . . . .

6.2 In the first step (upper left) we add a chirp and laser modulation. We show only one modulation wavelength, so the chirp effect is small. After the first chicane, the modulation is over-bunched (zoom, upper right). A second chirp reverses the first chirp (zoom, middle left, shows reversed slope in phase space). After more acceleration, the second chicane revives bunching (middle right). Note the compression of the wavelength by $|\alpha|=10$, and the increase in modulation amplitude by $|\alpha| / g=2.5$. We find strong bunching past the 5 th harmonic (bottom). . . . . . . . . . . . . . . . . . . .

6.3 Courant-Snyder parameters for 3D elegant simulations showing $\beta$-functions (solid and dotted lines) and dispersion ( $\eta_{x}$, dashed-dotted line). The weak second chicane is designed to minimize ISR effects. Following the second chicane, a third linac section increases the electron energy while simultaneously decreasing the $\beta$ function, enhancing the radiation power. . . .

6.4 a) Particle phase space for elegant simulation of parameters in Table 7.1. The two horizontal stripes of higher density are signatures of a sinusoidal modulation. A third-harmonic accelerating cavity produces the flat central portion of the beam. b) A zoom of the phase space shows the compressed modulation bunched to optimize higher harmonics. The vertical stripes ('standing up' the modulation) in phase space produce sharp density spikes that drive harmonic generation. The $T_{566}$ component of the transfer matrix causes slight scalloping. . .

6.5 Bunching factor at two wavelengths with $0.15 \%$ bandwidth from elegant [6] simulation (parameters in Table 7.1). Bunching is lower than for the 1D case because of 2nd order effects (e.g. emittance and $T_{566}$ ), ISR and finite laser radius. . . . . . . . . . . . . . 
6.6 To demonstrate the emittance cancelation effect, we track a longitudinal delta slice (zero length) following the laser modulation. Emittance effects increase the slice length before the second chicane, $\mathrm{BC} 2$, (top left, $x$ vs. $z$ ), but the off-crest accelerating section introduces a chirp to the beam (bottom left, energy vs. $z$ ). The second chicane then re-compresses the bunch by the compression factor, $|\alpha|$ (center). Following the final accelerator section, L3, the slice starts to spread out again (right). Bunch head is to the left. . . . . . . . . . . . .

6.7 Wide bandwidth bunching for two simulations with a 0.01 degree phase shift (solid and dotted blue lines). An FEL would pick out a narrow $\Delta \lambda / \lambda=0.15 \%$ bandwidth, leaving lower bunching (narrow peak, red dot-dash line).To calculate the bunching factor at a position $z_{0}$ and bandwidth $\Delta \lambda / \lambda$, we sum the phases of all particles in the region $z_{0}-\lambda / 2 \Delta \lambda<z<$ $z_{0}+\lambda / 2 \Delta \lambda$. The high baseline in the wide bandwidth curves is due to the relatively low number of particles per bunching calculation when $\lambda / \Delta \lambda$ is small. A bunched sine wave makes a sawtooth that may either be right-leaning, as in Fig. 6.4, or left-leaning, depending on the sign of the effective dispersion, $R_{56}^{(T)}$. In our simulation, $R_{56}^{(T)}$ changes as a function of longitudinal beam position, resulting in the double peak seen in the wide bandwidth bunching. 89

6.8 Double-horn energy distribution, left, from a sinusoidal modulation. Approximating a sawtooth with two frequencies gives the more uniform energy distribution at right. . . . . . . . .

7.1 An illustration of harmonic SSMB for $H=3$. At top, we show particles in phase space at the modulator. Each turn around the ring, particles slip forward or backward from dispersion, but the distribution is stationary for a periodic modulation. At bottom, at an intermediate point in the ring (1/H the way around), the microbunches are spaced by $\lambda_{\text {in }} / H$, i.e. the beam is

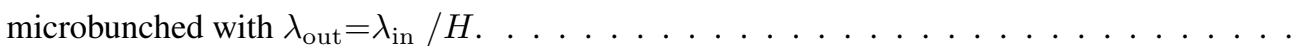

7.2 Example schematic for a two-stage system. A laser cavity and two undulators of length $L_{u}$ and $1.9 L_{u}$ modulate the electron beam at opposite ends of a storage ring. SSMB from the modulation and dispersion produces coherent light in a radiator. RF modules could replace the laser modulation to produce long wavelengths, and an additional radiator could be placed at the same distance in front of $L_{u} \ldots \ldots \ldots \ldots \ldots \ldots \ldots$

7.3 Phase space for harmonic SSMB, with one-stage modulation on left and two-stage modulation on right. The two-stage modulations provide a cleaner phase space with shorter stable regions, but the single modulation also creates stacked, periodic stable islands. . . . . . . . .

7.4 Example of an sawtooth manipulation to a coasting beam. Modulation has $V_{a}=V_{b}=0.05$, $R_{56}^{(a)}=1$ and $R_{56}^{(b)}=200$. After $2 \times 10^{5}$ loops, with damping of $\delta=10^{-4}$, unmodulated equilibrium energy spread of $10^{-4}$, and initial energy spread of $5 \%$, we find bunching at the 10th harmonic. Phase space of one wavelength is shown at upper left (after a full turn) and upper right (after $R_{56}^{(a)}$ ). The bunching profile is bottom left with the fourier transform bottom right. . . . . . . . . . . . . . . . . . . . . . 
7.5 Illustration of the Echo mechanism for a sawtooth modulation. We have chosen modulations $V_{a}=-V_{b}=10 \sigma_{\delta}$ to make the scheme easier to follow. The first dispersive section filaments the beam. The second modulation and dispersive sections then individually bunch the filaments. The harmonic is determined by the vertical separation of filaments (upper right), which will become horizontal separation after the final dispersive section (bottom right). We may change the number of filaments independently of the harmonic number. . . . . . . . . .

7.6 Illustration of the Echo mechanism for a sine modulation. We have chosen modulations $\bar{V}_{a}=-\bar{V}_{b}=2 \sigma_{\delta}$, and set $R_{56}^{(a)}$ for the 10th harmonic. Because a sine has two zero crossings $\left(h_{a}= \pm V k\right.$ ), we will have two different solutions for $R_{56}^{(b)}$, giving two different harmonics $H_{ \pm}$. For the parameters above, we find $H_{+}=10$ and $H_{-} \approx 8 \ldots \ldots \ldots \ldots$

7.7 Frequency-beating drives SSMB in multiple configurations. At left, modulations are in separate stages, and stable fixed points survive only where the phases overlap. At center, beating the laser prior to modulation can also drive SSMB if the electrons rely on the modulation to replace energy lost to synchrotron radiation. At right, the one-stage scheme is identical to a two-stage scheme with no dispersion between modulations. . . . . . . . . . . . . . . . .

7.8 Example of a long wavelength beating modulation with $b=10$. On the left, the two stage modulation affects particles throughout the beam, but stable fixed points can only survive near the center where the phases overlap. On the right, in a one stage modulation the two lasers beat together before interacting with the beam, and the modulation has little effect where the phases cancel. The one stage modulation still results in bunching when the combined modulation amplitude is less than the radiation loss (black line). Because the electrons rely on the modulation to replace energy lost to radiation, stable islands only survive in the center where the modulation is greater than the radiation loss. . . . . . . . . . . . . .

7.9 Phase space for frequency beating (with $b=10$ ) for both two-stage modulation (left) and onestage modulation (right). For two-stage modulations, the largest stable regions exist where the phases overlap. In the one-stage case, we find phase space stable islands throughout $\lambda_{\text {out }}=b \lambda_{\text {in }}$, but radiative energy loss removes the side islands. . . . . . . . . . . . . . . 104

7.10 In a system without RF, the modulation both drives SSMB and replaces the energy loss due to synchrotron radiation. The stable islands of the one-stage modulation are centered at the intersection of the synchrotron radiation (black line) and the combined beating modulation (blue line), with the intersection points highlighted by the red circles. Modulating with just one of the two beating wavelengths (green, dotted line) will modulate the stable islands by the level of the orange circles. We can see that this modulation chirps the stable islands, because of a relative phase slippage. A dispersive region can then compress the bunches. The sine wave shown (green) has the larger of the two beating wavelengths. Were we to have chosen the shorter wavelength, the chirp would have the opposite sign. . . . . . . . . . . .

7.11 Simulation of a two-stage manipulation to produce SSMB. After 10 million revolutions, we find bunches stacked at steady-state in energy space (top left). After an additional dispersive section of $R_{56} / 15$, we find SSMB at the 15 th harmonic (top right). The density profile is shown bottom left, with the Fourier transform bottom right. . . . . . . . . . . . . . . . 106 
8.1 Comparison of code to analytical result for straight motion and motion at an angle. The dotted line shows the case, $\sigma_{x}=2, \sigma_{z}=1$ and $\theta=\pi / 4 \ldots \ldots \ldots \ldots$. . . . . . . . 113

8.2 Benchmark for circular motion in the CSR limit. We compare our numerical code to the analytical solution $($ Eq. 8.26) . . . . . . . . . . . . . . . . . . . . . . . . . 114

8.3 CSR check for undulator motion. . . . . . . . . . . . . . . . . . . . 116

8.4 Comparison of undulator space charge at $\gamma$ to straight motion at $\gamma_{z}$. . . . . . . . . . . 117

8.5 1D FEL simulation for an 80nm bunch (LCLS parameters) when space charge produces a relative energy spread of $\sigma_{\delta} / \delta=10^{-3}$ at saturation (dashed line). The resulting power is slightly lower than the result without space charge $\gamma$ (solid line). When the space charge effect increases to $\sigma_{\delta} / \delta=1.65 \times 10^{-3}$ (dotted line), the power diminishes considerably. . . 118

8.6 Phase shifts (dashed line) compensate for space charge effects and recover the resonant condition at the front of the bunch even with strong space charge $\left(\sigma_{\delta} / \delta=2.5 \times 10^{-3}\right.$ at saturation). The phase shifts (every $3 \mathrm{~m}$ ) are not frequent enough to keep the correct phase for the 3rd harmonic (green lines) . . . . . . . . . . . . . . . . . . . . .

9.1 Fundamental radiation power from phase shifts. Phases done 3 ways: numerical optimization every $1 \mathrm{~m}$ (dark green), numerical optimization every $3 \mathrm{~m}$ (light green), phase scan every 1 m (red). The fourth line (blue) has no phase shifts. . . . . . . . . . . . . . . . . . . 125

9.2 Comparison of taper and equivalent phase shifts every $3 \mathrm{~m}$ in $1 \mathrm{D}$ simulations for a Seeded FEL (left) and SASE FEL (right). In both cases, the phase shift is less effective for the third harmonic, due to the relatively larger phase errors. . . . . . . . . . . . . . . . . 126

9.3 Phases determined by phase scan, numerical optimization and taper equivalent (Seeded case). 127

9.4 Taper for Seeded and SASE cases. Simulation using GINGER's 3D code. . . . . . . . . . . 128

9.5 Power for normal (black), tapered (red) and phase-shifted (blue) LCLS-like SASE FEL, 3-D simulation. GINGER simulation uses 12 phase shifts between undulator sections (every $3.8 \mathrm{~m}$ ). 129 


\section{Chapter 1}

\section{Introduction}

In the following work we consider a range of topics relating to electron microbunching in radiation sources. We define microbunching as density modulations within an electron bunch, driving coherent radiation at wavelengths shorter than the bunch. To illustrate the importance of microbunching, we first consider the radiation from an incoherent bunch of length, $L$, much longer than the radiation wavelength, $\lambda_{r}$. Assuming the electrons are evenly but randomly distributed, we expect each electron to radiate independently (i.e. with random phase), and the total radiation, $P_{r}$, to scale linearly with the number of electrons in the bunch, $N$. In contrast, in a short bunch, $L \ll \lambda_{r}$, the electrons all radiate with uniform phase. We then expect the radiation amplitudes to add constructively, and the power to scale as the square of the number of electrons, $P_{r} \propto N^{2}$. With $N$ as large as a billion, the enhancement due to coherence can be enormous.

For microbunching, we consider a long bunch $\left(L \gg \lambda_{r}\right)$ containing a periodic density modulation. As a toy model, we assume the bunch consists of periodic microbunches separated by $\lambda_{r}$. Despite the long bunch, $L \gg \lambda_{r}$, the electrons radiate with uniform phase, and consequently we still expect coherent radiation with $P_{r} \propto N^{2}$. We will refer to this phenomenon - coherence at a wavelength $\lambda_{r} \ll L-$ as microbunching.

To quantify the concept of microbunching at a wavevector $k \equiv 2 \pi / \lambda_{r}$, we define the bunching factor

$$
f(k) \equiv \frac{1}{\sqrt{N}} \sum_{i} e^{i k z_{i}}
$$

where the sum is over all longitudinal electron positions, $z_{i}$. (In Chapter 3 we extend this definition to arbitrary directions by generalizing to a vector $k \rightarrow \boldsymbol{k}$.) For either radiation sources or diagnostics, we are interested in the total radiated power, $P_{r}=P_{1} N|f(k)|^{2}$, with $P_{1}$ the power radiated by a single electron. We have chosen the normalization for $f(k)$ so that for random electron positions (i.e. shot noise), we find bunching amplitude squared $|f(k)|^{2}=1$, and for perfect bunching (all electrons with identical phase), we find $|f(k)|^{2}=N$.

The classic example of an electron-based coherent radiation source is the FEL [7]. In an FEL, a continuous stream of electrons travels through a long line of undulator magnets. The feedback between the electrons and the resulting radiation creates a beam microbunched at the undulator's resonant wavelength,

$$
\lambda_{r}=\frac{\lambda_{u}}{2 \gamma^{2}}\left(1+K^{2} / 2\right)
$$

with undulator period $\lambda_{u}$, undulator strength parameter $K$, and electron energy given by the relativistic $\gamma$ 
factor. The microbunched beam radiates coherently, producing radiation orders of magnitude brighter than that found in a standard undulator. (A full description of the FEL process can be found in, for example, [8].)

To start the FEL microbunching process, the electrons generally require a small initial level of either microbunching or radiation. (This is not strictly true; theoretically the seed may also come from energy modulation.) Ideally, an external laser source would either trigger the FEL or generate a pre-modulation in the electron beam (see e.g. [9] or Chapter 6). However, a key advantage of the FEL is the ability to tune to wavelengths beyond the reach of conventional lasers, and so by definition no convenient laser source exists. At such wavelengths, the shot noise of the beam itself can trigger the FEL in a process known as Self Amplified Spontaneous Emission (SASE) [10, 11]. Though we will not limit ourselves to FEL microbunching, we use this division of seeded and SASE microbunching to organize the topics of the following chapters. In the first half, we consider shot-noise driven microbunching; Chapter 3 addresses the microbunching instability (MBI), Chapter 2 extends the MBI analysis to the regime of noise suppression, and finally in Chapters 4 and 5 we report experimental results from the Linac Coherent Light Source (LCLS), the world's first hard $\mathrm{X}$-ray laser. In the second half, we consider new schemes for seeding light sources; Chapter 6 describes a scheme for seeding a linac-driven light source, Chapter 7 extends the seeding concept to a storage ring, and Chapter 8 addresses a potential pitfall of seeded beams. The last chapter discusses an alternative method of optimizing FEL power (relating tapered undulators to phase shifts) that can increase energy extraction from both seeded and shot noise microbunching, and relates to gain length measurements of undulator lines with gaps. Finally, an appendix includes detailed calculations to relate 3D and 1D results used in the chapters on noise suppression and MBI (Chapters 2 and 3).

For the harried reader, we present a brief summary of each chapter in the following sections.

\subsection{Noise Suppression}

In a bunch of random (uncorrelated) electrons, the longitudinal density contains white noise fluctuations, commonly called shot noise. While shot noise may be beneficial (e.g. as the driving force behind SASE FELs), the same density fluctuations may adversely affect FEL operation (e.g. the MBI). Shot noise also competes with external modulations in the operation of seeded FELs [12, 13]. Recent papers have proposed schemes to suppress the noise level below that of shot noise to aid the FEL process [14, 15, 16]. In Chapter 2 we use the approach of [17] to study noise evolution. We consider a simple model system of a generic selfinteraction, $h$, which changes the particle energies, followed by a dispersive region, $R_{56}$, which converts the change in energy to change in position. We show that for a broad class of interactions, it is possible to suppress density fluctuations below the shot noise level, and we provide 1D and 3D simulations to confirm the result.

\subsection{Microbunching Instability}

In a high-brightness linear accelerator, as found in a high-gain FEL, impedance effects along the accelerator can drive a periodic microbunching instability that degrades the electron beam qualities $[6,18,19,20,21,22$, 23, 24]. Longitudinal space charge (LSC) forces may dominate microbunching gain at wavelengths shorter than the electron bunch $[21,22,23,24]$. Evidence of optical wavelength microbunching has been seen at 
LCLS, where optical transition radiation (OTR) screens downstream from the high-brightness injector have recorded COTR from an uncompressed electron bunch [1]. At such short modulation wavelengths (less than $1 \mu \mathrm{m})$, electron shot noise is the most probable source of the initial density fluctuations required to start the instability.

Venturini noted [25] that the one-dimensional (1D) model of LSC impedance used in previous studies $[21,22,23,24]$ may fail at very short wavelengths, when the longitudinal shot noise modulations are not transversely uniform. In Chapter 3 we present a six-dimensional (6D) analysis of LSC microbunching starting from shot noise. Using a simplified machine model motivated by the LCLS setup, we calculate the microbunching gain factor for short modulation wavelengths observed at small angles relative to the longitudinal direction. We also compare these results with the LCLS COTR observations [26].

\subsection{LCLS Gain Length and Harmonics Results}

The Linac Coherent Light Source (LCLS) is the world's first hard x-ray laser, producing radiation 10 billion fold brighter than any other X-ray source in the world. LCLS achieved first lasing in April of 2009, and now operates as a user facility with photon energies ranging from $550 \mathrm{eV}$ to $9 \mathrm{keV}$ [5]. In Chapter 4 we present gain length measurements, which provide a metric for the health of the FEL microbunching process. We describe the methods used to measure gain lengths and post-saturation power growth, and present results of the first gain length measurements for LCLS.

Radiation at the fundamental wavelength of the FEL dominates in the experimental beamlines, but nonnegligible levels of radiation at higher harmonics are also present. These harmonics may be desirable as a source of harder x-rays, but may also contribute backgrounds to user experiments. In Chapter 5 we present measurements of the second and third harmonic content in the FEL at a range of photon energies. We measure the harmonic dependence on the electron beam quality, and compare the results to simulations. We also measure the photon energy cutoff of the soft x-ray mirrors to determine the extent to which higher harmonics reach the experimental stations.

\subsection{Compressed Harmonic Generation}

A Free Electron Laser (FEL) [7] can theoretically produce fully coherent x-rays, a promising tool for the fields of physics, chemistry and biology. However, current x-ray FELs in use or under construction rely on SASE FELs, which are saddled by long saturation lengths and poor longitudinal coherence. In contrast, FELs 'seeded' by optical or UV lasers promise full coherence and shorter FEL lengths. One leading seeded FEL scheme is high gain harmonic generation (HGHG) [9, 27]. However, single-stage HGHG requires high laser power (expensive and potentially problematic for the FEL process), and is limited to wavelengths around 20 $\mathrm{nm}$ [28]. Multiple-stage HGHG can reach shorter wavelengths, but is more technically challenging. A recent and promising seeding scheme is echo enabled harmonic generation (EEHG), which manipulates hidden structure in phase space to produce high harmonic seeding [29, 30].

Seeding the electron beam prior to bunch compression, which we will call compressed harmonic generation $(\mathrm{CHG})$, is an alternative approach $[31,32,33,34]$. In Chapter 6 we discuss a variation on CHG in which dispersion from the compression stage smears out the longitudinal modulation, but the structure 
remains imprinted in phase space and can be revived later by a second dispersive region [33, 35].

We give an analytical description of the two-chicane CHG scheme, and extend the simulations of [35] to include higher energies and smaller $\beta$-functions (desirable for sending the beam through a radiator). The major drawback of compressed seeding is the need to preserve fine phase space structure across long accelerator regions. However, we show that the second dispersive region reverses second order effects from the preceding accelerator section. Finally, we consider the technical requirements for effective transport of the phase space modulation.

\subsection{Steady-State Microbunching}

In a radiation source driven by coherent electrons, particles group into microbunches spaced at the wavelength of the desired light. The process may start from shot noise (as in the SASE FEL process) or from seeding (as in $\mathrm{CHG}$ ), but in either case the resulting coherent light can be orders of magnitude brighter than that of an equivalent incoherent source.

For high average power light sources, duty-cycle shares the stage with coherence. Linac driven FELs use each electron pulse once, leading to low duty cycles. (Energy recovery linacs, or ERLs, reach high duty cycles by recovering the electron energy [36,37, 38].) Storage rings, by contrast, naturally provide $\mathrm{MHz}$ repetition rates, and fully filled rings can provide $\mathrm{CW}$ radiation. However, storage rings do not generally support sustained microbunching (MB).

Ideally, we would produce a ring with both high duty cycle and coherent microbunching. Past proposals have suggested employing FELs attached to either ERLs or storage rings. In Chapter 7, we propose to modulate electrons in a storage ring with either RF or optical radiation, thereby driving MB during each pass through the ring's radiator. Though the electrons may appear smeared elsewhere in the ring, the MB is permanent at the radiator, so we consider this steady-state microbunching (SSMB). The result is a coherent radiation source with $\mathrm{MHz}$ to $\mathrm{CW}$ repetition rate. We present a mechanism for SSMB and show simulation results for several example cases.

\subsection{Longitudinal Space Charge}

Current enhanced SASE (eSASE) was proposed as a method to improve peak power, timing, and pulse length control in SASE FELs [39]. The scheme uses an optical laser and dispersive region to seed submicron current spikes in the electron bunch. In eSASE, the spikes do not drive coherent radiation themselves (as this radiation would be at very long wavelengths). Rather, the current spikes lase more efficiently than the lower current regions, providing short bursts of 'unseeded' SASE FEL radiation synched to the optical laser pulse (i.e. coherent control). Coherent control is particularly useful for pump-probe experiments with $\mathrm{X}$-ray FELs, and eSASE has been proposed as an upgrade to LCLS [40]. However, the sharp current spikes also exacerbate longitudinal space charge (LSC) induced energy spread in the electron beam. While LSC is generally suppressed in electron beams by the relativistic Lorentz factor, $\gamma=1 / \sqrt{1-\beta^{2}}$, Geloni et al. showed that the suppression is more accurately given by $\gamma_{z}=1 / \sqrt{1-\beta_{z}^{2}}$, with average longitudinal velocity, $\beta_{z}$ [41]. In the LCLS undulators, $\gamma_{z} \ll \gamma$, and the LSC may have a strong effect on the FEL process. In Chapter 8 we confirm Geloni's result with a numerical integration of the retarded electromagnetic 
potentials.

In Chapter 8, we use a 2-D numerical approach to calculate the longitudinal self-field of an electron bunch. In the first section, we describe the approach and derive the longitudinal component of the interaction for arbitrary motion. We express the equations in polar coordinates to facilitate a numerical code written in MATLAB. We then solve the equations for several simple examples, including a bunch traveling in uniform, circular and sinusoidal motion. We use these examples to benchmark the code against known solutions, and then finally confirm Geloni's expression for LSC of a Gaussian bunch in an undulator.

\subsection{Phase Shift Equivalence to Undulator Taper}

Despite the projected six orders of magnitude increase in peak power during the SASE process, some XFEL applications, including single molecule imaging, may require still higher photon flux [42]. Exponential power growth is no longer possible once the FEL reaches saturation (when the bunching factor is maximal), but the total FEL pulse energy may still increase linearly along the undulator; the electrons simply continue to radiate as long as the microbunched electrons remain at the optimal phase relative to the radiation.

Tapering is a well known method for increasing power past saturation by shifting the undulator $K$ value to offset the electrons' loss of energy. However, we can also view the undulator taper as an offsetting phase shift (relative to the FEL radiation), so we may achieve the same goal by simply inserting electron phase shifters (e.g. a chicane to delay the electrons).

Varfolomeev et al. first described the use of phase shifts to enhance power in 1998 [43]. (We learned of this work following our study.) In Chapter 9, we undertake a detailed analytical and numerical study of enhancing FEL power with phase shifters. We explore the relation between phase shifts and undulator tapers to calculate optimal phase shifts for SASE FELs in the saturation regime and apply these shifts to simulations

with LCLS-like parameters. The phase shift method, while equivalent to tapering the undulator parameter, provides an independent knob to maximize the FEL performance. Conversely, we may apply the same result to employ an undulator taper as a phase shift. We demonstrate the effect by studying the gain length of the LCLS beam line with undulators removed (section 4.3.4). 


\section{Chapter 2}

\section{Shot Noise Suppression for Electron}

\section{Beams}

\subsection{Introduction}

In a bunch of random (uncorrelated) electrons, the longitudinal density contains white noise fluctuations, commonly called shot noise. While shot noise drives Self-Amplified Spontaneous Emission (SASE) Free Electron Lasers (FELs), the same density fluctuations may adversely affect FEL operation. For example, the microbunching instability, thought to originate from shot noise, can incapacitate diagnostics and degrade FEL performance [18, 20, 1, 44, 45, 46, 47]. Shot noise also competes with external modulations in the operation of seeded FELs $[12,13]$. Recent papers have proposed schemes to decrease the noise level below that of shot noise to aid the FEL process or for other applications $[14,15,16]$. In this chapter we use the approach of [17] to study the evolution of noise as the beam travels through a system with interactions between the electrons as well as dispersive regions. To simplify the analysis, we consider a model system of a generic self-interaction, $h$, which changes the particle energies, followed by a dispersive region, $R_{56}$, which converts the change in energy to change in position (Fig. 2.1). We show that for a broad class of interactions, it is possible to suppress density fluctuations below the shot noise level, and we provide 1D simulations to confirm the result.

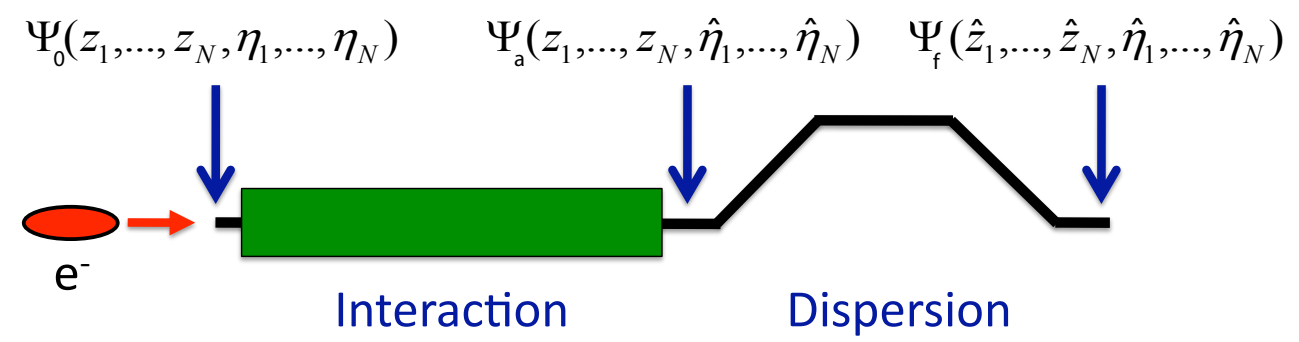

Figure 2.1: Schematic of our model system. Starting with an initial electron distribution function, $\Psi_{0}(z, \eta)$, the interaction and dispersive regions produce a final distribution function, $\Psi_{f}(\hat{z}, \hat{\eta})$. The dispersion may be positive or negative. 


\subsection{Analytical Model}

\subsubsection{Noise Factor}

To characterize the level of noise at a wavevector, $k$, we define the noise factor

$$
F(k, s) \equiv \frac{1}{N} \sum_{j, l} e^{i k\left[z_{j}(s)-z_{l}(s)\right]}
$$

where $z_{j}(s)$ is the longitudinal bunch coordinate of particle $j$ at position $s$ in the accelerator, and $N$ is the number of particles in the beam. We note that the noise factor can equivalently be defined by $F(k, s) \equiv$ $N|b(k, s)|^{2}$, with the bunching factor $b(k, s) \equiv \sum_{j} \exp \left[i k z_{j}(s)\right] / N$.

The noise factor, $F(k, s)$, is a measure of the correlations between particle coordinates at wavevector $k$. If the particle positions are uncorrelated, we find the expectation value of shot noise, $\langle F(k, s)\rangle=1$. On the other hand, if the positions are strongly correlated at wavevector $k$, we find $\langle F(k, s)\rangle \sim N$, with $N \gg 1$ generally; such correlated (or 'bunched') beams are found at the output of an FEL, and as the result of the microbunching instability $[46,47]$. We may also consider the case of an anti-correlated (or 'quiet') beam, with $\langle F(k, s)\rangle<1$, below the shot noise level. In this chapter, we investigate the possibility of producing quiet beams.

Though the noise factor is defined as a function of accelerator position, $s$, we are particularly interested in the noise level at the output of our system, $F\left(k, s_{f}\right)$. Starting from an initial distribution function at $s_{0}$, we would like to determine the resulting final noise level at $s_{f}$.

To facilitate an analytical solution, we will study the simplified system of Fig.2.1. We assume the particle distribution is a function of position in the bunch, $z(s)$, and relative, normalized energy, $\eta(s) \equiv[E(s)-$ $\left.E_{0}\right] / E_{0}$, with average beam energy, $E_{0}$. Though both $z$ and $\eta$ are functions of $s$, we are primarily interested in the initial and final coordinates, so for brevity we define $z, \eta \equiv z\left(s_{0}\right), \eta\left(s_{0}\right), \hat{z}, \hat{\eta} \equiv z\left(s_{f}\right), \eta\left(s_{f}\right)$ and $F(k) \equiv F\left(k, s_{f}\right)$. We can then describe the system as follows. We start with a simple $N$-particle initial distribution of particles, $\Psi_{0}\left(z_{1}, \ldots, z_{N}, \eta_{1}, \ldots, \eta_{N}\right)$. After an interaction period, the energies are modified, giving distribution $\Psi_{a}\left(z_{1}, \ldots, z_{N}, \hat{\eta}_{1}, \ldots, \hat{\eta}_{N}\right)$. A dispersive region (assumed to have zero interaction), then changes the longitudinal positions, giving final distribution $\Psi_{f}\left(\hat{z}_{1}, \ldots, \hat{z}_{N}, \hat{\eta}_{1}, \ldots, \hat{\eta}_{N}\right)$.

\subsubsection{Expectation Value of Noise Factor}

To calculate the expectation value, we break $\langle F(k)\rangle$ into incoherent $(j=l)$ and coherent $(j \neq l)$ portions. First, we treat the incoherent portion. With $j=l$, the phases cancel and we find $N$ terms, all equal to 1 , giving

$$
\langle F(k)\rangle_{S N}=1
$$

which is simply the noise level due to shot noise.

Next, we calculate the coherent portion. To find the expectation value at the final accelerator position, we integrate $F(k)$ over the final particle distributions, $\Psi_{f}\left(\hat{z}_{1}, \ldots, \hat{z}_{N}, \hat{\eta}_{1}, \ldots, \hat{\eta}_{N}\right)$. In general, $\Psi_{f}$ may be a complicated function of all $2 N$ variables. However, if we assume the electrons are initially uncorrelated, then we can write the initial distribution function as

$$
\Psi_{0}\left(z_{1}, \ldots, z_{N}, \eta_{1}, \ldots, \eta_{N}\right)=\prod_{i}^{N} f^{(1)}\left(z_{i}, \eta_{i}\right)
$$


with the single particle distribution functions for a beam with Gaussian energy spread of $\sigma_{\eta}$ and uniform longitudinal density of length $L$ given by

$$
f^{(1)}(z, \eta)=\left\{\begin{array}{ll}
\frac{e^{-\eta^{2} / 2 \sigma_{\eta}^{2}}}{\sqrt{2 \pi} \sigma_{\eta} L} & \text { for }-L / 2<z<L / 2 \\
0 & \text { elsewhere }
\end{array} .\right.
$$

We then express the final coordinates in terms of the initial coordinates $(\hat{z}, \hat{\eta} \rightarrow z, \eta)$, and integrate over the product of $N$ simple initial distributions, $f^{(1)}$.

In the interaction region, we assume the bunch is longitudinally frozen $(z, \eta \rightarrow z, \hat{\eta})$, and likewise in the dispersive region we assume there is zero interaction $(z, \hat{\eta} \rightarrow \hat{z}, \hat{\eta})$. To further simplify the calculation, we ignore any transverse effects. (The validity of the $1 \mathrm{D}$ approximation will depend on the interaction of interest.) Our resulting map from initial to final coordinates then is

$$
\begin{aligned}
& \eta_{j} \rightarrow \hat{\eta}_{j}=\eta_{j}+\sum_{i=1}^{N} h\left(z_{j}, z_{i}\right) \\
& z_{j} \rightarrow \hat{z}_{j}=z_{j}+R_{56} \hat{\eta}_{j}
\end{aligned}
$$

with dispersive strength $R_{56}$, and $h\left(z_{j}, z_{i}\right)$ the change in energy of particle $j$ due to the interaction with particle $i$. We can now write the coherent portion of $F(k)$ in terms of the initial coordinates, and integrate over each single particle distribution, $\Psi_{i} \equiv f^{(1)}\left(z_{i}, \eta_{i}\right)$, to find the expectation value

$$
\begin{aligned}
&\langle F(k)\rangle_{C} \approx N \int_{-L / 2}^{L / 2} d z_{1} d \eta_{1} \ldots \int_{-L / 2}^{L / 2} d z_{N} d \eta_{N} \Psi_{1} \ldots \Psi_{N} \\
& e^{i k\left(z_{1}-z_{2}+R_{56}\left(\eta_{1}-\eta_{2}\right)+R_{56}\left[\sum_{i}^{N} h\left(z_{1}, z_{i}\right)-\sum_{m}^{N} h\left(z_{2}, z_{m}\right)\right]\right)}
\end{aligned}
$$

where we have assumed the $N^{2}-N \approx N^{2} \gg 1$ coherent terms of the sum in Eq. 2.1 are identical, and we have chosen $j=1, l=2$ without loss of generality.

Our approach (following [17]) will be to explicitly separate the $z_{1}, z_{2}$ terms. We assume the interaction depends only on the distance between the particles, $h\left(z_{1}, z_{2}\right)=h\left(z_{1}-z_{2}\right)$, so we change variables, $z_{1}, z_{2}, z_{l}, z_{m} \rightarrow \zeta, Z, \tau_{l}, \tau_{m}$ with $\zeta \equiv z_{1}-z_{2}, Z \equiv\left(z_{1}+z_{2}\right) / 2$, and $\tau_{l, m} \equiv z_{l, m}-z_{2}$. Finally, we assume that the interaction is nonzero only within a characteristic distance, $L_{h}$, which is much shorter than the bunch length, $L$. We can then integrate over $Z$ and $\eta_{1} \ldots \eta_{N}$ to find

$$
\begin{aligned}
& \langle F(k)\rangle_{C}=n_{0} e^{-k^{2} R_{56}^{2} \sigma_{\eta}^{2}} \int_{-\infty}^{\infty} d \zeta\left[\frac{1}{L^{N-2}} \int d \tau_{3} \ldots \int d \tau_{N}\right. \\
& \left.e^{i k\left(\zeta+R_{56}[h(\zeta)-h(-\zeta)]+R_{56} \sum_{i=3}^{N}\left[h\left(-\tau_{i}+\zeta\right)-h\left(-\tau_{i}\right)\right]\right)}\right]
\end{aligned}
$$

where we have defined the 1D particle density $n_{0} \equiv N / L$ and we have used $L_{h} \ll L$ to both ignore edge effects and set the $\zeta$ integral limits to infinity. First, we note that the $N-2$ integrals over $\tau_{i}$ are separable and identical. Second, we assume $k R_{56} h \ll 1$ so we can linearize the exponentials, yielding

$$
\langle F(k)\rangle_{C} \approx n_{0} e^{-k^{2} R_{56}^{2} \sigma_{\eta}^{2}} \int_{-\infty}^{\infty} d \zeta e^{i k \zeta}\left[1+\Gamma_{1}(\zeta)\right]\left[1+\frac{1}{N} \Gamma_{2}(\zeta)\right]^{N-2}
$$


with definitions

$$
\begin{aligned}
& \Gamma_{1}(\zeta) \equiv i k R_{56}[h(\zeta)-h(-\zeta)]+\ldots \\
& \Gamma_{2}(\zeta) \equiv n_{0} \int_{-\infty}^{\infty} d \tau\left(i k R_{56}[h(-\tau+\zeta)-h(-\tau)]\right. \\
& \left.\quad \quad-\frac{k^{2} R_{56}^{2}}{2}[h(-\tau+\zeta)-h(-\tau)]^{2}+\ldots\right)
\end{aligned}
$$

where we have expanded $\Gamma_{1}$ and $\Gamma_{2}$ in powers of the small parameter, $k R_{56} h$. For $\Gamma_{1}$, the term linear in $k R_{56} h$ is nonzero, so we drop all higher order terms. However, from our assumption of a long bunch, the linear order terms in $\Gamma_{2}$ cancel after the integration, so we must also keep the quadratic term for $\Gamma_{2}$. Combining the two square terms, $\int d \tau h(-\tau)^{2}=\int d \tau h(-\tau+\zeta)^{2}$, we find

$$
\begin{aligned}
& \Gamma_{1}(\zeta) \approx i k R_{56}[h(\zeta)-h(-\zeta)] \\
& \Gamma_{2}(\zeta) \approx n_{0} k^{2} R_{56}^{2} \int_{-\infty}^{\infty} d \tau\left[h(-\tau+\zeta) h(-\tau)-h(-\tau)^{2}\right] .
\end{aligned}
$$

We may be tempted to drop $\Gamma_{2}$, because it is second order in $k R_{56} h$. However, $\Gamma_{2}$ is also raised to the power of $N$, and with $N \gg 1$ generally, $\Gamma_{2}$ may even be the dominant term (as for the microbunching instability, see e.g. $[46,47])$. In this chapter, we keep both terms, and will see that noise suppression occurs when $\Gamma_{1}$ and $\Gamma_{2}$ are comparable.

\subsubsection{Analytical Expression: Weak Interaction}

If we consider a weak interaction under the stronger assumption, $\Gamma_{2} \ll 1$, we can solve for the noise level analytically. Adding in the shot noise term again and expanding Eq. 2.8, we find

$$
\langle F(k)\rangle \approx 1+n_{0} e^{-k^{2} R_{56}^{2} \sigma_{\eta}^{2}}\left\{\int_{-\infty}^{\infty} d \zeta e^{i k \zeta}\left[\Gamma_{1}(\zeta)+\Gamma_{2}(\zeta)\right]+2 \pi \delta(k)\right\} .
$$

We are interested in $k \neq 0$, and so will drop the $\delta$ function. (The $\delta$ function arises from our assumption of $L \rightarrow \infty$. For finite $L$, we will have a term that is nonzero for $k<1 / L$, but even so our focus is on much shorter wavelengths.)

We can now identify the three regimes for $\langle F(k)\rangle$. For zero interaction, we are left with only the leading shot noise term, $\langle F(k)\rangle=1$, which is simply the white noise of an uncorrelated bunch. The $\Gamma_{2}$ contribution is positive-definite, so for $\Gamma_{2} \gg \Gamma_{1}$, we find a correlated beam with $\langle F(k)\rangle>1$. Finally, for $\Gamma_{1} \sim \Gamma_{2}$, the term linear in $R_{56}$ cannot be neglected. If $R_{56}$ is chosen so that $\Gamma_{1}<0$, it is possible to create an anticorrelated beam, with the noise factor suppressed below the shot noise level, $\langle F(k)\rangle<1$. In this chapter we consider the third regime.

Identifying the $\zeta$ integral as a Fourier transform (FT), we rewrite the noise factor as

$$
\begin{aligned}
& \langle F(k)\rangle \approx 1+i n_{0} k R_{56}[\tilde{h}(k)-\tilde{h}(-k)] e^{-k^{2} R_{56}^{2} \sigma_{\eta}^{2}} \\
& +n_{0}^{2} k^{2} R_{56}^{2} F T\left\{\int_{-\infty}^{\infty} d \tau\left[h(-\tau) h(-\tau+\zeta)-h(-\tau)^{2}\right]\right\} e^{-k^{2} R_{56}^{2} \sigma_{\eta}^{2}}
\end{aligned}
$$

where $\tilde{h}(k)$ denotes $\operatorname{FT}\{h(\tau)\}$. We drop the second term in the remaining integral because it has no $\zeta$ dependence, and so its Fourier transform is nonzero only for wavelengths longer than the bunch $(k<1 / L)$. 
The first term is the autocorrelation of $h(\tau)$, which has Fourier transform $|\tilde{h}(k)|^{2}$, yielding

$$
\begin{aligned}
\langle F(k)\rangle & \approx 1-2 n_{0} k R_{56} \operatorname{Im}[\tilde{h}(k)] e^{-k^{2} R_{56}^{2} \sigma_{\eta}^{2}} \\
& +n_{0}^{2} k^{2} R_{56}^{2}|\tilde{h}(k)|^{2} e^{-k^{2} R_{56}^{2} \sigma_{\eta}^{2}} .
\end{aligned}
$$

If the energy spread is small $\left(\sigma_{\eta} \rightarrow 0\right)$, and the interaction has purely imaginary Fourier transform, $\tilde{h}(k)$, we can write the noise factor as a perfect square

$$
\langle F(k)\rangle \approx(1-\Upsilon)^{2} \text { with } \Upsilon \equiv n_{0} k R_{56} \operatorname{Im}[\tilde{h}(k)]
$$

We suppress the noise factor below the shot noise level when the suppression parameter is in the range $0<\Upsilon<2$ and the noise disappears completely for $\Upsilon=1$. (We note that partial noise suppression is possible even if the interaction contains a real component.) We are particularly interested in interactions that can be approximated as step functions near $\zeta=0: h(\zeta) \rightarrow A H(\zeta)+$ const, with Heaviside function $H$, and interaction strength, $A$. For such interactions, we find $\tilde{h}(k) \propto 1 / k$ for high frequencies, so that $\Upsilon$ is independent of $k$. We are then able to simultaneously suppress bunching at a wide range of frequencies.

We can draw a broad lesson from Eq. 2.13; a quiet beam is attainable from any interaction with primarily imaginary Fourier transform, e.g. from step function interactions (for $k \neq 0$ ). We will treat the special cases of space charge and undulator interactions later, but here emphasize that any interaction with imaginary Fourier transform will suffice, and any step function interaction will give broadband noise suppression. For example, the wake from a linac with periodic structures also satisfies these conditions [48]. We have assumed negligible energy spread here; see the appendix for a discussion of the effect of energy spread on noise suppression.

For a physical interpretation of the requirement for imaginary Fourier transform, we consider a test particle in front of localized density spike of width $1 / k$. If $h(\tau)>0$ for $\tau>0$, the test particle will receive positive energy change. A positive dispersive region then causes the test particle to move forward and away from the dense region. Likewise, a test particle at the back of a dense region $(\tau<0)$ loses energy relative to the front particle for $\tilde{h}(k)$ imaginary, and moves backward and away in a positive dispersive region. The end result is a reduction in the density spike and thus a reduction in the noise. If $h(\tau)<0$ for $\tau>0$, as is the case for an undulator, we have the identical argument, but require negative dispersion. The process is illustrated in Fig. 2.2.

\subsubsection{Numerical Approximation: Strong Interaction}

For stronger interactions, we may not be able to approximate $\Gamma_{2} \ll 1$. If it is not possible to evaluate Eq. 2.8 analytically for an arbitrary $h$, we can carry out the integrals numerically. Using the less stringent approximation $\Gamma_{2} \ll N$ (satisfied even for simulation parameters with relatively small $N$ ) we take $(1+$ $\left.\Gamma_{2} / N\right)^{N} \approx \exp \left(\Gamma_{2}\right)$ to obtain

$$
\langle F(k)\rangle=1+n_{0} e^{-k^{2} R_{56}^{2} \sigma_{\eta}^{2}} \int_{-\infty}^{\infty} d \zeta e^{i k \zeta} e^{\Gamma_{2}(\zeta)}\left[1+\Gamma_{1}(\zeta)\right]
$$

For physical interactions, $\Gamma_{1} \rightarrow 0$ as $\zeta \rightarrow \infty$, so the second term, $e^{\Gamma_{2}(\zeta)} \Gamma_{1}(\zeta)$, converges and can be integrated numerically. We cannot directly integrate the first term, $\exp \left[\Gamma_{2}(\zeta)\right]$, because the $h^{2}(-\tau)$ in Eq. 2.10 has no $\zeta$ dependence; in the limit $\zeta \rightarrow \infty$, we find $\Gamma_{2}(\zeta) \rightarrow \bar{\Gamma}_{2} \neq 0$, and the integral diverges. However, the 


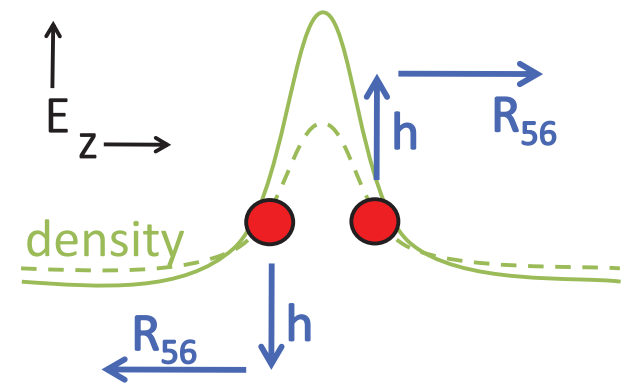

Space Charge

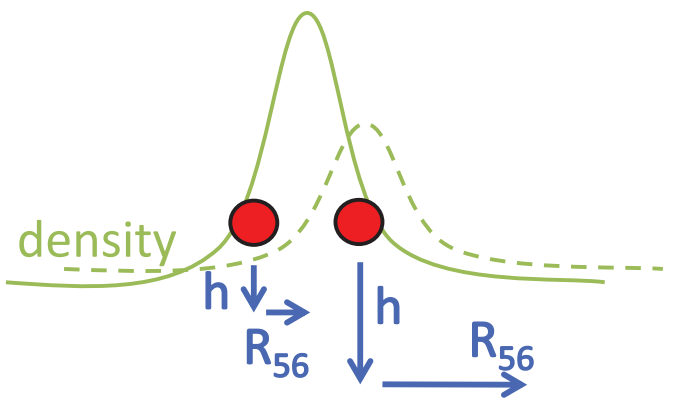

Undulator

Figure 2.2: Schematic of an interaction near a density spike (solid green line). At left, for the space charge case, particles in the front half of the spike gain energy, while particles in the back half lose energy, and in positive dispersion, the density spike shrinks (dotted green line). We have a similar result for an interaction due to undulator radiation (right). At high frequency (spike much shorter than undulator resonant wavelength), all particles lose energy, but following a dispersive region with negative $R_{56}$ we still find a reduction in the density spike (dotted green line).

divergence occurs only for $k=0$; otherwise, $\bar{\Gamma}_{2} \exp [i k \zeta]$ integrates to zero (which is why we dropped the $h^{2}(-\tau)$ term from Eq. 2.12). Following the same reasoning here, with

$$
\bar{\Gamma}_{2}=-n_{0} k^{2} R_{56}^{2} \int_{-\infty}^{\infty} d \tau h(-\tau)^{2}
$$

we explicitly remove the constant term, $\exp \left[\bar{\Gamma}_{2}\right]$ to find

$$
\begin{aligned}
\langle F(k)\rangle & =1+n_{0} e^{-k^{2} R_{56}^{2} \sigma_{\eta}^{2}} \int_{-\infty}^{\infty} d \zeta \\
& {\left[\left(e^{\Gamma_{2}(\zeta)}-e^{\bar{\Gamma}_{2}}\right) \cos (k \zeta)+i e^{\Gamma_{2}(\zeta)} \Gamma_{1}(\zeta) \sin (k \zeta)\right] }
\end{aligned}
$$

where we've used Eq. 2.10 to see that $\Gamma_{1}(\zeta)$ and $\Gamma_{2}(\zeta)$ are respectively odd and even functions of $\zeta$. We can then integrate Eq. 2.17 numerically.

\subsection{Space Charge Case}

\subsubsection{Space Charge Interaction}

So far we have not specified the interaction term, constraining only that the energy change, $h$, is a function of $\zeta$, the distance between the particles. We now consider the Coulomb interaction between two particles. We assume the interaction occurs over a distance $L_{a}$ in the accelerator, during which the particles are frozen longitudinally. We consider a 1D system, treating the particles as uniform, rigid sheets of charge with radius $a$, valid in the limit $a \gg \gamma / k$ [25]. To calculate the relative change in energy due to the longitudinal E-field $\left(E_{z}\right)$, we integrate over the sheets of source and test particles,

$$
h_{s c}(\zeta)=\frac{\operatorname{sgn}(\zeta)}{\gamma m_{e} c^{2}} \frac{q^{2} L_{a}}{4 \pi \epsilon_{0} S^{2}} \int_{0}^{a} \int_{0}^{a} \int_{0}^{2 \pi} \int_{0}^{2 \pi} \frac{r_{1} d r_{1} r_{2} d r_{2} d \theta_{1} d \theta_{2}(\gamma \zeta)}{\left[(\gamma \zeta)^{2}+r_{1}^{2}+r_{2}^{2}-2 r_{1} r_{2} \cos \left(\theta_{1}-\theta_{2}\right)\right]^{3 / 2}}
$$


with average particle energy, $\gamma m c^{2}$, electron charge, $e$, area of sheet, $S=\pi a^{2}$, and

$$
\operatorname{sgn}(\zeta) \equiv \begin{cases}1 & \zeta>0 \\ 0 & \zeta=0 \\ -1 & \zeta<0\end{cases}
$$

One of the $\theta$ integrals trivially gives a factor of $2 \pi$, and the remaining integrals can be solved numerically to produce the interaction $h_{s c}(\zeta)$ shown in Fig. 2.3. We note that the interaction will go to zero for $\zeta \gg a / \gamma$, as required in our derivation of Eq. 2.7. In the limit of infinite sheets $(a \rightarrow \infty)$, the $E_{z}$ field is simply

$$
\left|E_{z}\right|=\frac{e}{2 S \epsilon_{0}}
$$

so that the interaction causes an energy change per charge, $e$, of

$$
h_{s c}(\zeta)=\frac{e^{2} L_{a}}{2 \epsilon_{0} S \gamma m_{e} c^{2}} \operatorname{sgn}(\zeta)=\frac{2 \pi r_{e} L_{a}}{S \gamma} \operatorname{sgn}(\zeta),
$$

with classical electron radius $r_{e} \equiv e^{2} / 4 \pi \epsilon_{0} m_{e} c^{2}$.

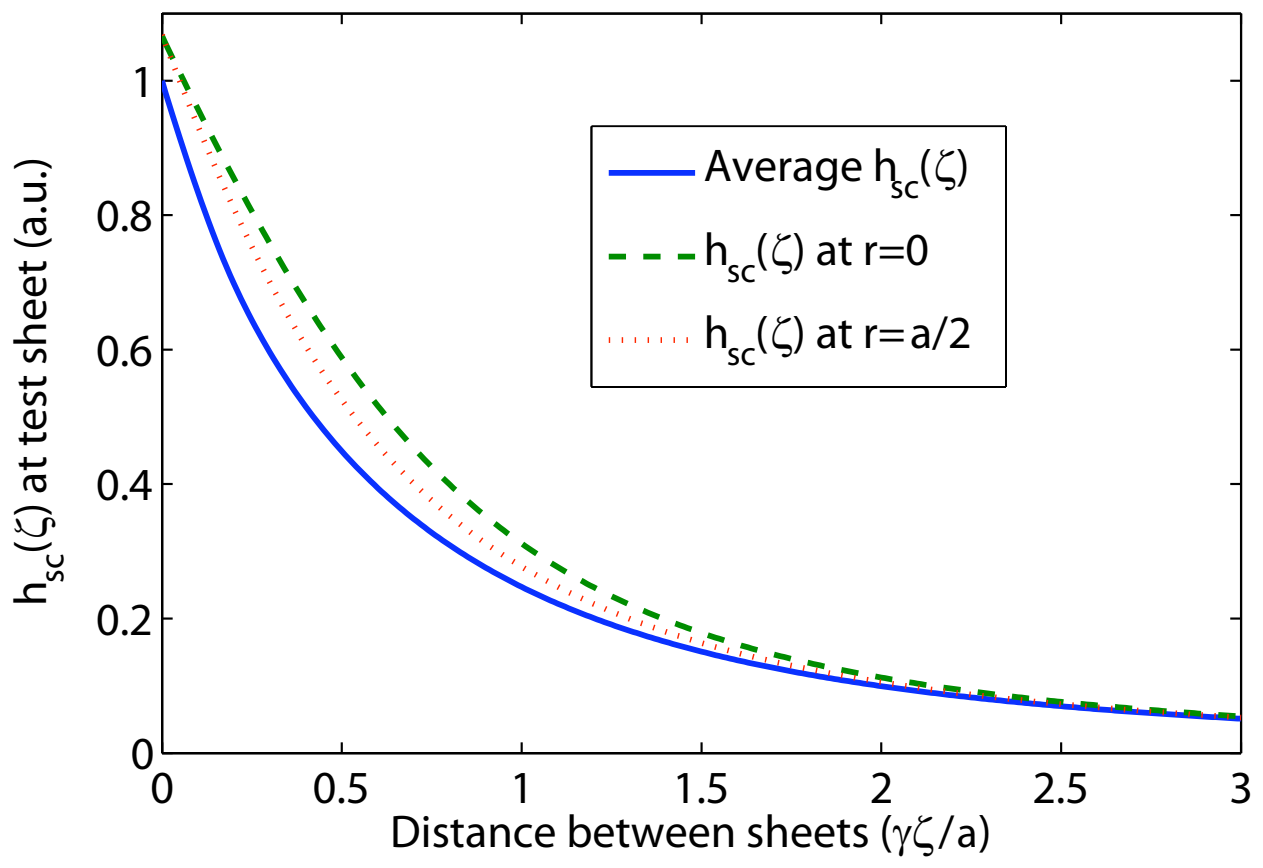

Figure 2.3: Space charge from a source sheet produces a change in energy $\left(h_{s c}\right)$ in a test sheet located at a distance $\zeta$. We calculate the energy modulation to the test sheet by averaging over the entire sheet (solid blue curve). Though $E_{z}$ (and thus $h_{s c}$ ) is not constant everywhere in the test sheet, we note that there is relatively little variation near the center of the sheet, as can be seen from $h_{s c}$ evaluated at radius $r=0$ (dashed green line) and $r=a / 2$ (dotted red line). 


\subsubsection{Space Charge Fourier Transform}

In the simplified case of infinite sheets, the step function at $\zeta=0$ dominates $\tilde{h}(k)$, and we find a purely imaginary Fourier transform,

$$
\tilde{h}(k)=\frac{i A_{s c}}{k}
$$

with definition

$$
A_{s c} \equiv \frac{4 \pi r_{e} L_{a}}{S \gamma}
$$

Following Eq. 2.14, we then define the suppression parameter for space charge, $\Upsilon_{s c} \equiv n_{0} R_{56} A_{s c}$, and we expect broadband suppression for $\Upsilon_{s c}=1$.

In the finite sheet model, when $\zeta \gg a / \gamma$ the interaction falls off as $1 / \zeta^{2}$. The cutoff for $h(\zeta)$ as $\zeta \rightarrow \infty$ determines the noise suppression at low frequencies; the approximation of $\tilde{h}(k) \propto 1 / k$ breaks and we expect suppression to be frequency dependent for small $k$. Averaging the energy modulation across the disc gives (see e.g. $[49,50]^{1}$ )

$$
\tilde{h}(k)=\frac{i A_{s c}}{k}\left[1-2 I_{1}(k) K_{1}(k)\right],
$$

with modified Bessel functions $I_{1}(x), K_{1}(x)$. As $k \rightarrow \infty$, we find $\tilde{h}(k) \rightarrow i A_{s c} / k$, reproducing the result for the infinite sheet (Eq. 2.22). However, as $k \rightarrow 0$, we find $\tilde{h}(k) \rightarrow 0$, and we expect weaker noise suppression.

\subsubsection{Space Charge Simulation}

To check our analytical result, we simulate the interaction between particles in a 1D code. We load $N$ particles randomly within a bunch length $L$, with initial energy spread, $\sigma_{\eta}$. A particle at location $z_{0}$ interacts with all particles within the range $z_{0}-L_{h}<z<z_{0}+L_{h}$, and we choose the interaction distance $L_{h}$ so that $L \gg L_{h} \gg a / \gamma$. To avoid edge effects from a finite bunch, we enforce periodic boundary conditions on the interaction. Following the interaction, the longitudinal positions shift according to $\hat{z}=z+R_{56} \hat{\eta}$, where the relative energy $\hat{\eta}$ is solely determined by the interactions of the first stage. We can then calculate the noise factor (or equivalently the FFT) of the resulting distribution, though even by eye it is apparent we have suppressed high frequency noise (Fig. 2.4). In the limit of a cold beam, the 1D space charge interaction results in regularly spaced particles, each separated by the local inverse density, $1 / n_{0}$ (Fig. 2.5).

We check the analytical solution (Eqs. 2.13, 2.24) against the simulations in Fig. 2.6. For all space charge simulations, units of length are normalized to the sheet radius, $a$, and for now we assume zero initial energy spread, $\sigma_{\eta}=0$.

\subsubsection{Validity of $1 \mathrm{D}$ model}

Throughout the chapter we use a 1D model of sheet particles (sheets distributed with random longitudinal positions), so we would like to check that the resulting interaction, Eq. 2.24, is a reasonable approximation of a 3D distribution of particles. We may look to Ref. [25], which studies the difference between 1D and 3D models of longitudinal space charge in the high frequency limit. Though the 1D and 3D distributions of

\footnotetext{
${ }^{1}$ In Eq. 2 of Reference [50], the Bessel function should be $K_{1}$, not $K_{0}$.
} 

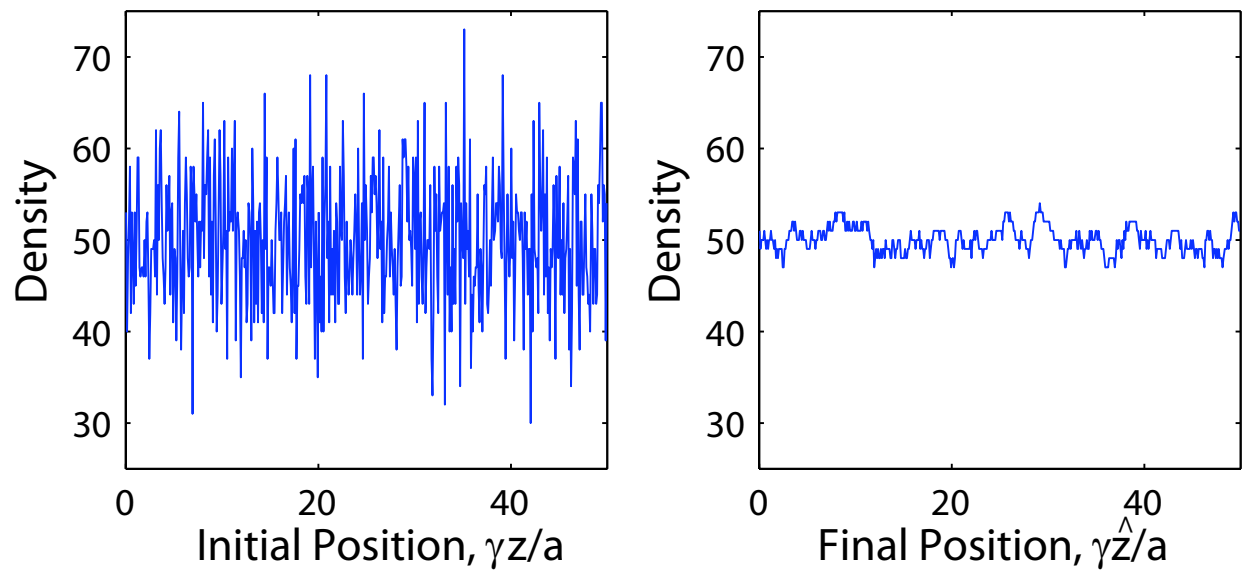

Figure 2.4: On the left we show a histogram of particle density for particles loaded with random longitudinal positions. At right, following the interaction and dispersive regions, we find a reduction in noise in the equivalent histogram. (Example has $n_{0} a=5 \times 10^{2}, \Upsilon=1$ ).

longitudinal fields diverge at high frequency (see Eqs. 9,11-13 from Ref. [25]), we find that when averaged transversely, the two models give approximately equal results (Section 2.11).

The assumption of rigid 1D sheets may also overestimate the noise suppression. Past work on noise suppression resulting from plasma oscillations has found that 3D models lead to weaker noise suppression $[14,15]$. In our 1D model we assume a rigid sheet of charge that moves uniformly due to the average longitudinal field, whereas in reality each particle moves independently. To check the validity of our 1D model, we have written a 3D version of the space charge simulation. We confirm the existence of noise suppression for $\Upsilon=1$, but with somewhat weaker level of suppression (Fig. 2.16).

\subsection{Undulator Radiation Case}

\subsubsection{Undulator Radiation Interaction}

As a second example, we consider the case of a beam traveling through an undulator. In the 1D limit, we can write down a simple, closed form solution for the interaction due to a helical undulator [51], providing a convenient system for studying noise suppression. For this reason, we neglect the space charge component in the following analysis, though we will see that in the absence of an amplifier [16] the space charge effect is generally dominant. We then find the undulator interaction

$$
h_{u}(\zeta)= \begin{cases}-A_{u}\left(1-\frac{\zeta}{N_{u} \lambda_{0}}\right) \cos k_{0} \zeta & 0<\zeta<N_{u} \lambda_{0} \\ 0 & \text { otherwise }\end{cases}
$$

with definition

$$
A_{u} \equiv 2 \pi \frac{e^{2} K^{2} N_{u} \lambda_{u}^{2}}{S \gamma^{3} m_{e} c^{2} \lambda_{0}}=4 \pi \frac{r_{e} L_{u}}{S \gamma} \frac{K^{2}}{1+K^{2}} .
$$




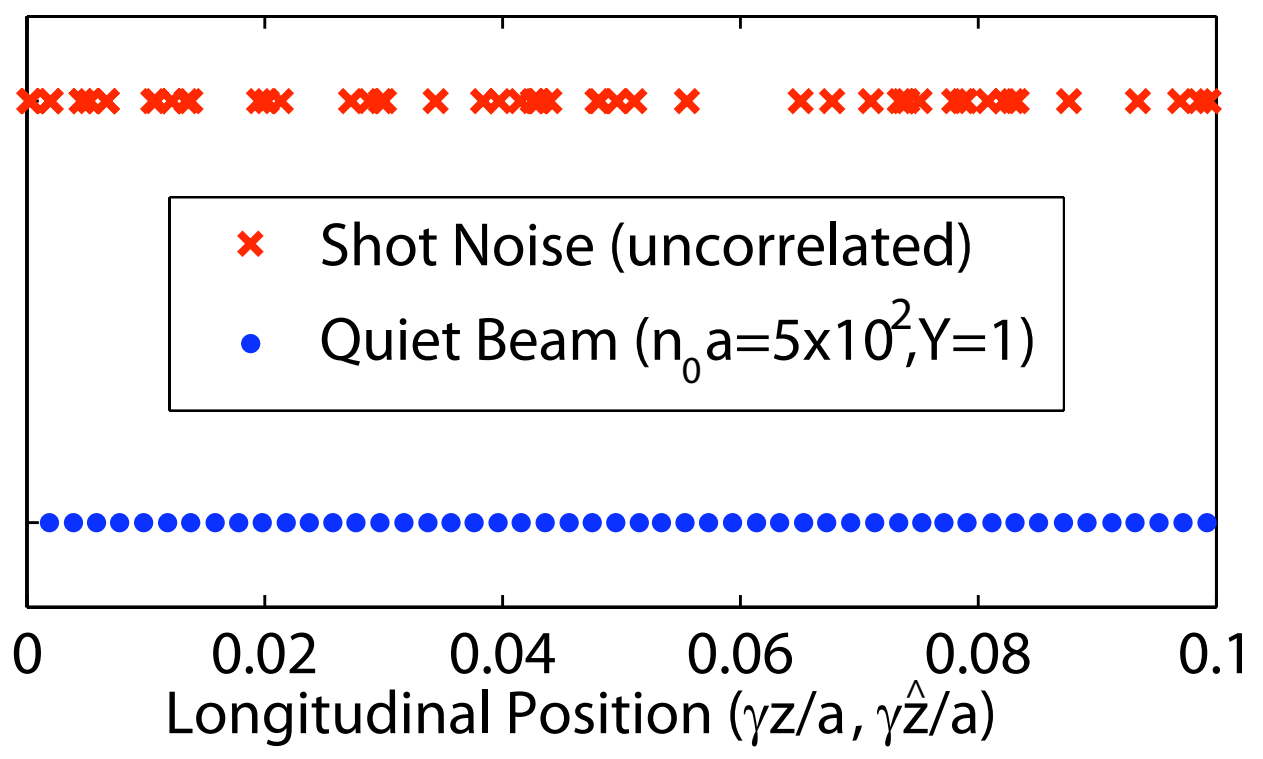

Figure 2.5: Longitudinal distribution of particles in simulation before $(\times)$ and after $(*)$ the noise suppression process. For $\sigma_{\eta} \ll 1 / R_{56} n_{0}$ and a $1 \mathrm{D}$ beam, it is possible to show that the initially uncorrelated distribution gives way to a regularly spaced beam with inter-particle spacing $1 / n_{0}$ (see section 2.9 ). The regular structure amplifies bunching at very high frequencies, $k=2 \pi n_{0}$ and its harmonics, while suppressing $F(k)$ at frequencies below $2 \pi n_{0}$.

undulator strength parameter, $K$, length, $L_{u}$, period, $\lambda_{u}$, and resonant wavelength, $\lambda_{0}$. This $1 \mathrm{D}$ expression is valid in the limit

$$
a \gg \frac{\gamma}{k \sqrt{1+K^{2}}}
$$

with $a$ the transverse beam size.

\subsubsection{Undulator Fourier Transform}

From Eq. 2.13, noise suppression originates from the imaginary component of the Fourier transform. For the undulator case,

$$
\begin{aligned}
\tilde{h}_{u}(k) & \equiv \int_{-\infty}^{\infty} d \zeta e^{i k \zeta} h_{u}(\zeta) \\
& =-A_{u} N_{u} \lambda_{0} \int_{0}^{1} d \bar{\zeta}(1-\bar{\zeta}) \cos \alpha \bar{\zeta} e^{i m \alpha \bar{\zeta}}
\end{aligned}
$$

with definitions $\bar{\zeta} \equiv \zeta / N_{u} \lambda_{0}, \alpha \equiv 2 \pi N_{u}$ and $m \equiv k / k_{0}$. Integrating gives

$$
\tilde{h}_{u}(k)=-i A_{u} N_{u} \lambda_{0}\left[\frac{m}{\left(m^{2}-1\right) \alpha}-i \frac{\left(1+m^{2}\right)\left(1-e^{i m \alpha}\right)}{\left(m^{2}-1\right)^{2} \alpha^{2}}\right]
$$

with $N_{u}$ assumed to be an integer. At high frequencies ( $m \gg 1$ ), we neglect the second term, and find a purely imaginary FT

$$
\tilde{h}_{u}(k) \approx-i \frac{A_{u}}{k} .
$$




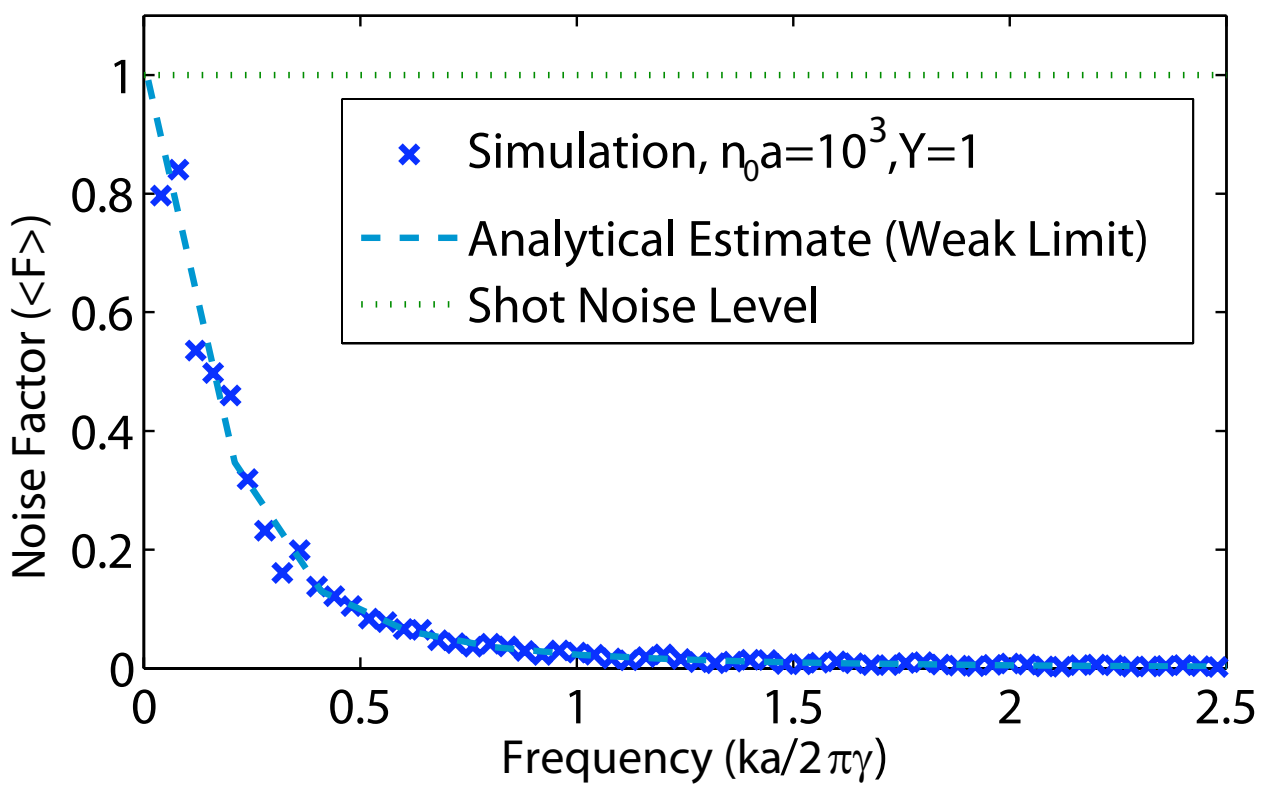

Figure 2.6: A comparison of simulation and analytical results shows noise suppression as a function of frequency. With $\Upsilon \approx 1$ at high frequency, we find strong suppression. At low frequencies ( $k \lesssim 2 \pi / a$ ), we no longer have $\tilde{h}_{u}(k) \propto 1 / k$, so suppression is weaker for the given parameters.

As in the space charge case, we use Eq. 2.14 to define the suppression parameter $\Upsilon_{u}=-A_{u} n_{0} R_{56}$. In general, $\tilde{h}_{u}(k)$ is not purely imaginary, as stipulated in Eq. 2.13. However, at high frequencies, the undulator interaction looks like a step function (with the purely imaginary Fourier transform in Eq. 2.30), and the physical picture in Fig. 2.2 applies here as well. Again, $\Upsilon_{u}$ has no $k$ dependence, so we expect broadband suppression.

At low frequencies, the approximation in Eq. 2.30 fails and the Fourier transform will be complex. If we take the limit of $m \rightarrow 1$, then from Eq. 2.29 we find

$$
\tilde{h}_{u}\left(k=k_{0}\right)=-A_{u} N_{u} \lambda_{0}\left(\frac{1}{4}+\frac{i}{4 \alpha}\right)
$$

which is approximately real. We then find $\langle F(k)\rangle \sim 1+\left|\tilde{h}_{u}\left(k_{0}\right)\right|^{2}$ and consequently expect bunching to increase at low frequencies. Note that $\left|\tilde{h}_{u}\left(k_{0}\right)\right|=\left(N_{u} \pi / 2\right) \Upsilon_{u}$, so for $N_{u} \gg 1$, we can expect an enhancement of $\sim N_{u}^{2} \pi^{2} / 4$ at the fundamental when $\Upsilon=1$.

It is interesting to note that at high frequencies, the undulator interaction is strictly weaker than space charge (Eq. 2.26 vs. Eq. 2.23). Because the interactions have opposite sign, the undulator would only act to dampen the noise suppression from space charge.

\subsubsection{Undulator Simulation}

To check our analytical result, we again run the simulation code but with the undulator interaction (Eq. 2.25) instead of space charge. We load $N$ particles randomly within a length $L \gg N_{u} \lambda_{0}$, and for the undulator case a particle at location $z_{0}$ interacts with all particles within the range $z_{0}-N_{u} \lambda_{0}<z<z_{0}$. 
The simulations confirm both the analytical solution (Eq. 2.13, valid for $\Gamma_{2} \ll 1$ ) and the numerical integral (Eq. 2.17). In all undulator simulations, we normalize units of length to the resonant wavelength, $\lambda_{0}$, and we assume zero initial energy spread, $\sigma_{\eta}=0$. (In section 2.10 we consider the effects of initial energy spread and energy modulation to the beam.)

\subsubsection{Undulator Numerical Integration}

While we already know the noise factor in the weak-interaction limit from Eq. 2.14, we would like to calculate $\Gamma_{2}$ explicitly to evaluate the numerical integral. For $\Upsilon \approx 1$, we find the weak interaction limit is equivalent to

$n_{0} \gg k^{2} L_{u}$. While the weak approximation is valid for many realistic examples, to facilitate simulations we use low particle numbers, where the approximation fails. For that reason, we use the numerical integration, Eq. 2.17, to check our simulations without the assumption of weak interaction.

Plugging the undulator interaction into Eq. 2.10 yields (see section 2.8)

$$
\begin{aligned}
\Gamma_{2}^{(u)}(\bar{\zeta}) & =\frac{k^{2} R_{56}^{2}}{L} A_{u}^{2} N_{u} \lambda_{0}\left(\frac{1}{6}+\frac{1}{4 \alpha^{2}}+\right. \\
& \frac{1}{12 \alpha^{3}}\left[\alpha(1-\bar{\zeta})\left[\alpha^{2}\left(\bar{\zeta}^{2}+\bar{\zeta}-2\right)-3\right] \cos (\alpha \bar{\zeta})\right. \\
& \left.\left.+3\left[\alpha^{2}(1-\bar{\zeta})-1\right] \sin (\alpha \bar{\zeta})\right]\right)
\end{aligned}
$$

Plugging into Eq. 2.16 gives constant term for the undulator interaction

$$
\bar{\Gamma}_{2}^{(u)}=\frac{n_{0} \alpha k_{0}\left(m R_{56} A_{u}\right)^{2}}{N}\left[\frac{1}{6}+\frac{1}{4 \alpha^{2}}\right],
$$

and then from Eq. 2.17 we find

$$
\begin{aligned}
\langle F(k)\rangle & =1+2 n_{0} \alpha e^{-k^{2} R_{56}^{2} \sigma_{\eta}^{2}} \\
& {\left[A_{u} m R_{56} \int_{0}^{1} d \bar{\zeta} e^{-N \Gamma_{2}^{(u)}}(1-\bar{\zeta}) \cos (\alpha \bar{\zeta}) \sin (m \alpha \bar{\zeta})\right.} \\
& \left.+\frac{1}{k_{0}} \int_{0}^{1} d \bar{\zeta}\left(e^{-N \Gamma_{2}^{(u)}}-e^{-N \bar{\Gamma}_{2}^{(u)}}\right) \cos (m \alpha \bar{\zeta})\right]
\end{aligned}
$$

which can be integrated directly. Simulations for the case of $N_{u}=1$ show good agreement with both the analytical result, Eqs. 2.13 and 2.29, and the numerical integration of Eq. 2.34, though as expected the analytical result fails for $n_{0} \sim k^{2} L_{u}$ (Figs. 2.7, 2.8). For a case with a longer undulator $\left(N_{u}=10\right)$, the numerical integration is essential for comparison with simulations (Fig. 2.9). At this point we can also explicitly confirm the result from Section 2.2 .3 by plugging $\tilde{h}(k)$ and $\Gamma_{2}^{(u)}(\bar{\zeta})$ back into Eqs. 2.8 and 2.10 (see appendix, Section 2.8).

\subsection{Example Parameters}

Though the focus of this chapter is strictly theoretical, we calculate the interaction strength for SLAC's Next Linear Collider Test Accelerator (NLCTA) to illustrate the scale of parameters involved. For the case of space charge over a length of $L_{a} \sim 10 \mathrm{~m}$ with beam cross section $S \sim 10^{-6} \mathrm{~m}^{2}$ and energy $\gamma m c^{2} \sim 100 \mathrm{MeV}$, we 


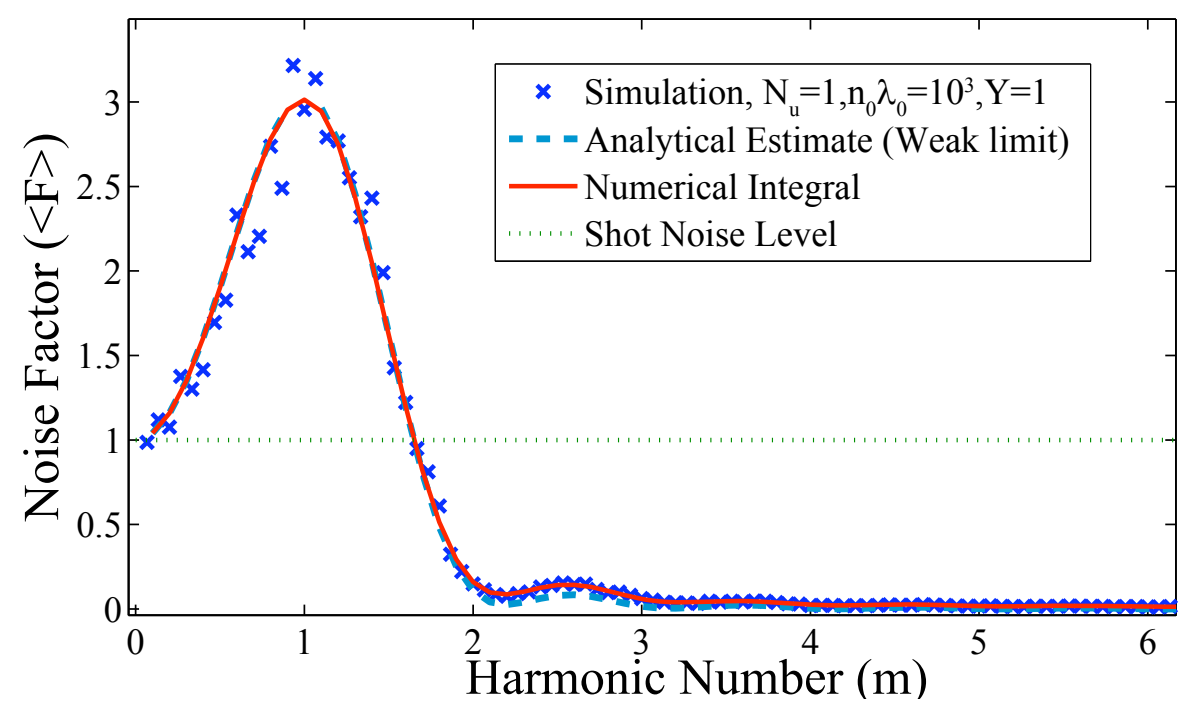

Figure 2.7: A comparison of simulation, analytical result and numerical integral shows noise suppression at high frequency for $\Upsilon=1$. At low frequencies ( $m \sim 1$ ), we find $\tilde{h}_{u}(k)$ is approximately real (Eq. 2.31), and bunching increases to $F(k) \approx 1-2 n_{0} k_{0} R_{56} \operatorname{Im}\left[\tilde{h}_{u}\left(k_{0}\right)\right]+\left(n_{0} k R_{56}\right)^{2}\left|\tilde{h}_{u}\left(k_{0}\right)\right|^{2} \approx 3$.

find $A_{s c} \equiv \frac{4 \pi r_{e} L_{a}}{S \gamma} \approx 2 \times 10^{-9}$. A beam of $20 \mathrm{~A}\left(n_{0}=4 \times 10^{11} \mathrm{~m}^{-1}\right)$, then needs $R_{56} \sim 2 \mathrm{~mm}$ to produce $\Upsilon=1$. We note that we are within the $1 \mathrm{D}$ limit even for optical wavelengths $\left(k_{0} \sigma / \gamma \gtrsim 25\right)$.

For the undulator radiation to dominate over the space charge interaction, we may use an amplifier, as proposed by Litvinenko [16]. The increase in the interaction strength also has the benefit of decreasing the required dispersion, $R_{56}$, allowing for larger energy spreads and higher frequency suppression. However, the larger modulation may increase the beam energy spread (see section 2.10).

\subsection{Conclusion}

We present a longitudinal 1D model of shot noise suppression for a simplified system of an interaction region followed by a dispersive region. In the limit of small energy spread $\left(\left|k R_{56} \sigma_{\eta}\right| \ll 1\right)$, interactions with primarily imaginary Fourier transforms can suppress the noise factor below the shot noise level. We work out the specific cases of undulator and space charge interactions, and confirm both results with a 1D simulation. We note that a wide range of imaginary impedances (e.g. linac wakefields) may also reduce shot noise. In the 1D limit with small energy spread, the suppression process may amplify bunching at very high frequencies near the inter particle spacing, $1 / n_{0}$.

\subsection{Acknowledgments}

We would like to thank K.J. Kim and R. Lindberg for helpful discussions. This work was supported by U.S. DOE Contract No. DE-AC02-76SF00515. 


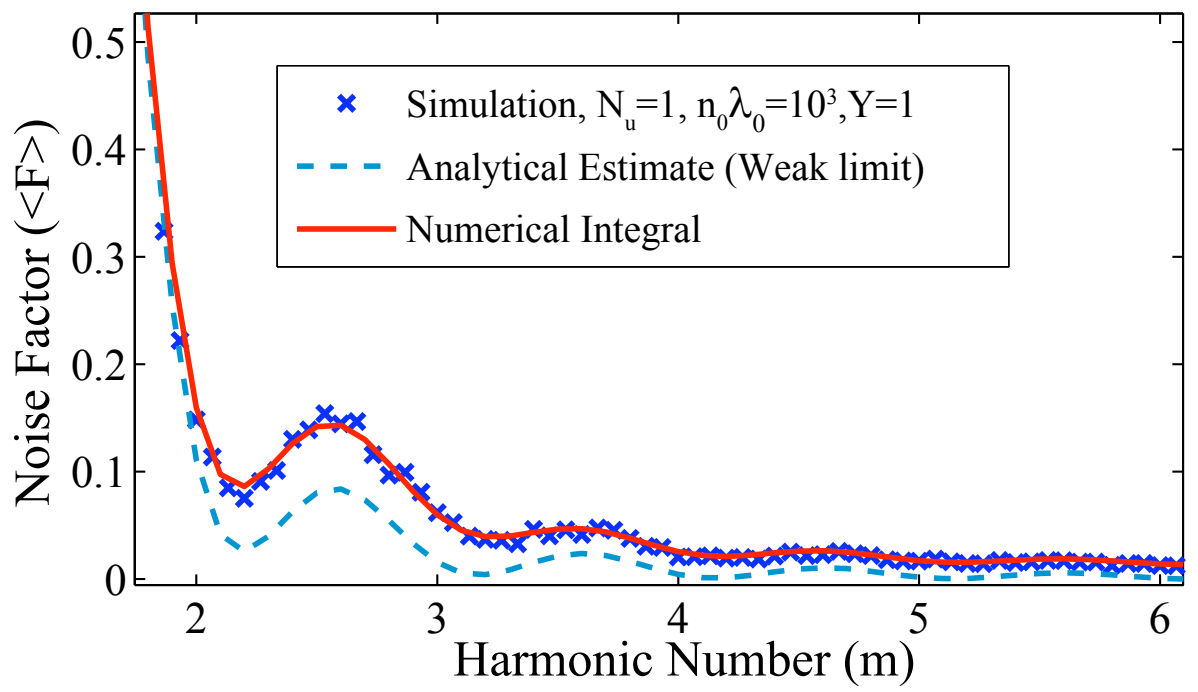

Figure 2.8: A close-up of Fig. 2.7 shows agreement with the analytical expression starts to fail for $m \gtrsim 2$, but the numerical integral matches well everywhere.

\subsection{Quadratic Term $\left(\Gamma_{2}\right)$ for Helical Undulator}

We calculate $\Gamma_{2}$ explicitly for use in the numerical integral (Eq. 2.34). Plugging Eq. 2.25 into Eq. 2.10 gives

$$
\begin{aligned}
\Gamma_{2}(\zeta) & =n_{0} k^{2} R_{56}^{2}\left[\int_{-N_{u} \lambda_{0}}^{0} d \tau h_{u}(-\tau)^{2}\right. \\
& \left.\left.-\int_{-N_{u} \lambda_{0}+\zeta}^{0} d \tau h_{u}(-\tau+\zeta) h_{u}(-\tau)\right]^{2}\right]
\end{aligned}
$$

where we've used $h_{u}(x)=0$ outside of the range $0<x<N_{u} \lambda_{0}$ to choose the integration limits. Plugging in for $h_{u}$, defining normalized variables $\bar{\tau} \equiv \tau / N_{u} \lambda_{0}, \bar{\zeta} \equiv \zeta / N_{u} \lambda_{0}$ and $\alpha \equiv 2 \pi N_{u}$, we have

$$
\begin{aligned}
& \Gamma_{2}(\bar{\zeta})=n_{0} k^{2} R_{56}^{2} A_{u}^{2} N_{u} \lambda_{0}\left[\int_{-1}^{0} d \bar{\tau}(1+\bar{\tau})^{2} \cos (\alpha \bar{\tau})^{2}\right. \\
& \left.-\int_{\bar{\zeta}-1}^{0} d \bar{\tau}(1+\bar{\tau}-\bar{\zeta})(1+\bar{\tau}) \cos (\alpha(\bar{\tau}-\bar{\zeta})) \cos (\alpha \bar{\tau})\right],
\end{aligned}
$$

We can then integrate to find

$$
\begin{aligned}
\Gamma_{2}(\bar{\zeta}) & =n_{0} k^{2} R_{56}^{2} A_{u}^{2} N_{u} \lambda_{0}\left[\frac{1}{6}+\frac{1}{4 \alpha^{2}}+\frac{1}{12 \alpha^{3}}\right. \\
& \left(\alpha(1-\bar{\zeta})\left[\alpha^{2}\left(\bar{\zeta}^{2}+\bar{\zeta}-2\right)-3\right] \cos (\alpha \bar{\zeta})+\right. \\
& \left.\left.3\left[\alpha^{2}(1-\bar{\zeta})-1\right] \sin (\alpha \bar{\zeta})\right)\right],
\end{aligned}
$$

where we've assumed an integer number of undulator periods, $N_{u}$, to simplify the trigonometric functions. Note that this expression is valid only for $0<\bar{\zeta}<1$; while Eq. 2.37 does not look symmetric about $\zeta=0$, from Eq. 2.10 we know that $\Gamma_{2}$ is an even function of $\bar{\zeta}$.

In the main text, we use $\Gamma_{2}$ to evaluate Eq. 2.17. We can also use $\Gamma_{2}$ to explicitly confirm the result from Section 2.2.3 for the high frequency undulator case by plugging Eq. 2.37 into Eq. 2.11. With $\Gamma_{2}$ an even 


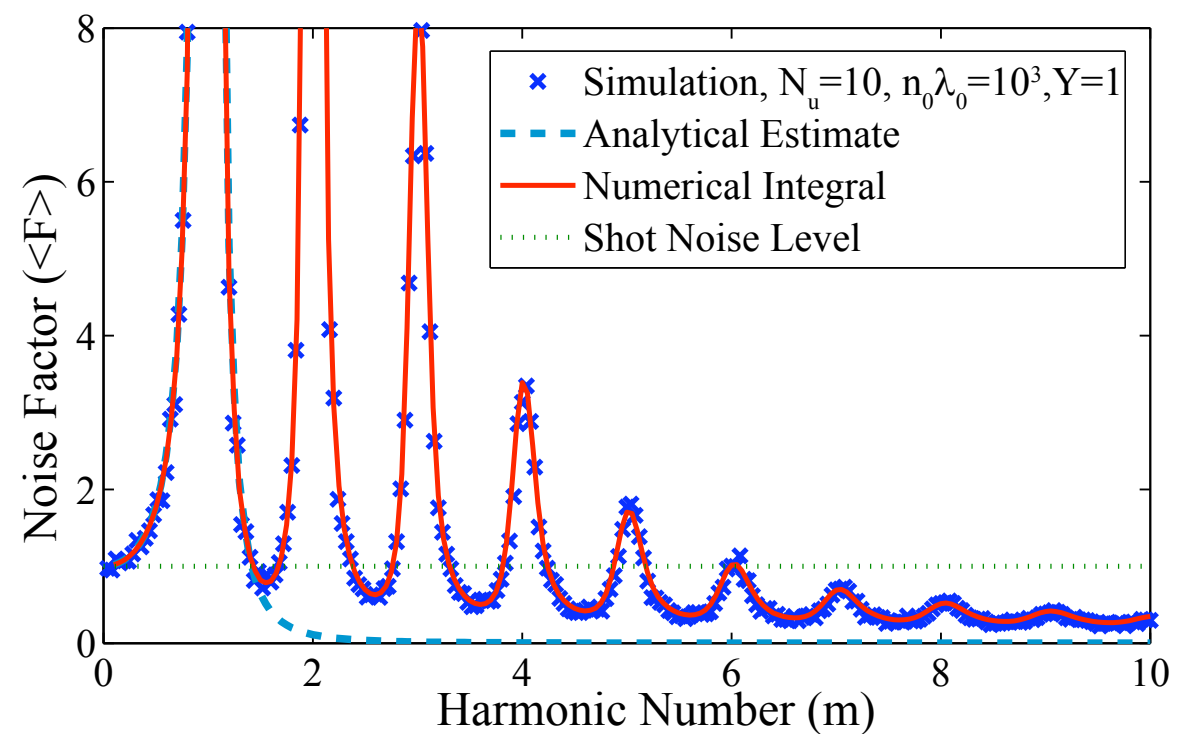

Figure 2.9: A simulation example for 10 undulator periods shows good agreement with the numerical integral.

function, we can write the third (quadratic) term from Eq. 2.11 as

$$
\left\langle F_{2}(k)\right\rangle \equiv 2 n_{0} e^{-k^{2} R_{56}^{2} \sigma_{\eta}^{2}} N_{u}^{2} \lambda_{0}^{2} \int_{0}^{L} d \bar{\zeta} \cos (m \alpha \bar{\zeta}) N \Gamma_{2}(\bar{\zeta})
$$

We are interested in $k \neq 0$, so as in Eq. 2.17, we subtract off the two constant terms. By definition, the product of interactions must disappear for $\bar{\zeta}>1$ (when at least one of $z_{1}$ and $z_{2}$ cannot interact with the test charge), so we set the upper limit to 1 and integrate to find

$$
\begin{aligned}
\left\langle F_{2}(k)\right\rangle & =-2 n_{0}^{2} k^{2} R_{56}^{2} A_{u}^{2} N_{u} \lambda_{0} e^{-k^{2} R_{56}^{2} \sigma_{\eta}^{2}} \frac{1}{4 \alpha^{4}\left(m^{2}-1\right)^{4}} \\
& {\left[2 m^{2} \alpha^{2}\left(m^{2}-1\right)^{2}+4\left(m^{2}+1\right)^{2}(\cos (m \alpha)-1)\right.} \\
& \left.+4 m \alpha\left(m^{4}-1\right) \sin (m \alpha)\right]
\end{aligned}
$$

In the limit $m \gg 1$ the result simplifies to

$$
\begin{aligned}
\left\langle F_{2}(k)\right\rangle & =n_{0}^{2} k^{2} R_{56}^{2} A_{u}^{2} N_{u}^{2} \lambda_{0}^{2} e^{-k^{2} R_{56}^{2} \sigma_{\eta}^{2}} \frac{1}{m^{2} \alpha^{2}} \\
& =\Upsilon_{u}^{2} e^{-k^{2} R_{56}^{2} \sigma_{\eta}^{2}}
\end{aligned}
$$

Adding in the first two terms of Eq. 2.13 and taking the limit of $m \gg 1$ and $\sigma_{\eta}=0$, we confirm $\langle F(k)\rangle=$ $\left(1-\Upsilon_{u}\right)^{2}$.

\subsection{Bunching at the Average Inter-Particle Spacing}

In the cold, sheet-beam limit (treating each particle as a sheet), we show that an interaction, $h(\zeta)$, results in nearly full bunching factor at the inter-sheet spacing if there is a step function at $\zeta=0$ (Fig. 2.5). To facilitate 


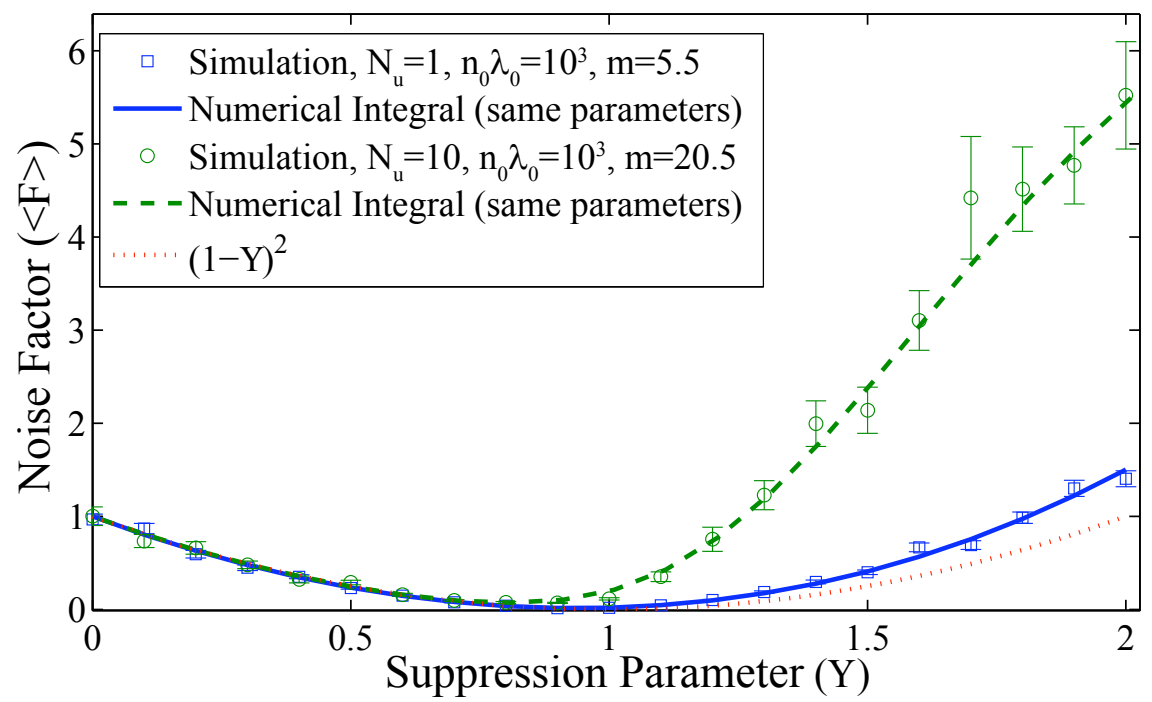

Figure 2.10: Simulations show the noise factor for both $N_{u}=1$ and $N_{u}=10$ as a function of $\Upsilon$. When the approximation $k^{2} N_{u} \lambda_{0} / n_{0} \gg 1$ is valid, the noise scales as $(1-\Upsilon)^{2}$. We have chosen $m$ so that $\tilde{h}_{u}(k)$ is approximately imaginary.

the calculation, we number our particles (sheets) such that $z_{i}<z_{k}$ if $i<k$. The energy change for particles $k$ and $k+1$ is given by

$$
\Delta E_{k}=\sum_{i=1}^{N} h\left(z_{k}-z_{i}\right), \quad \Delta E_{k+1}=\sum_{i=1}^{N} h\left(z_{k+1}-z_{i}\right) .
$$

To find the difference in energy modulations, $\Delta E_{k+1}-\Delta E_{k}$, we rewrite $\Delta E_{k+1}$ in terms of $\Delta E_{k}$,

$$
\begin{aligned}
\Delta E_{k+1} & =h\left(z_{k+1}-z_{k}\right)+\sum_{i \neq k}^{N} h\left(z_{k+1}-z_{k}+z_{k}-z_{i}\right) \\
& \approx h\left(\Delta z_{k}\right)+\sum_{i \neq k}^{N} h\left(z_{k}-z_{i}\right)+\Delta z_{k} \sum_{i \neq k}^{N} h^{\prime}\left(z_{k}-z_{i}\right) \\
& =\Delta E_{k}+\left[h\left(\Delta z_{k}\right)-h\left(-\Delta z_{k}\right)\right]+\Delta z_{k} \sum_{i \neq k}^{N} h^{\prime}\left(z_{k}-z_{i}\right),
\end{aligned}
$$

where we've defined the initial distance between particles, $\Delta z_{k}=z_{k+1}-z_{k}$, and assumed that the average spacing, $\langle\Delta z\rangle$, is small to make the Taylor expansion in the second step. (Specifically, we assume $[h(\zeta+$ $\Delta)-h(\zeta)] / h(\zeta) \ll 1$, everywhere except at the step function.) Hence the energy difference between the two neighboring particles is

$$
\Delta E_{k+1}-\Delta E_{k}=\left[h\left(\Delta z_{k}\right)-h\left(-\Delta z_{k}\right)\right]+\Delta z_{k} \sum_{i \neq k}^{N} h^{\prime}\left(z_{k}-z_{i}\right) .
$$

To compute the last sum, we replace the summation by an integration (assuming, as before, a uniform longitudinal distribution of particles in the beam). Skipping over the region where we've explicitly assumed there 
are no particles, for a longitudinally uniform beam we find

$$
\begin{aligned}
\sum_{i=1}^{N} h^{\prime}\left(z_{k}-z_{i}\right) & \approx n_{0}\left[\int_{-\infty}^{z_{k}^{-}} d z h^{\prime}\left(z_{k}-z\right)+\int_{z_{k+1}^{+}}^{\infty} d z h^{\prime}\left(z_{k}-z\right)\right] \\
& =n_{0}\left[\int_{\infty}^{0^{+}}(-d \zeta) h^{\prime}(\zeta)+\int_{-\Delta z_{k}^{+}}^{-\infty}(-d \zeta) h^{\prime}(\zeta)\right] \approx-n_{0}\left[h\left(0^{+}\right)-h\left(0^{-}\right)\right] .
\end{aligned}
$$

where we've approximated $h\left(\Delta z_{k}^{ \pm}\right) \approx h\left(0^{ \pm}\right)$. For an interaction with a step function at $\zeta=0$ of amplitude $A=\left[h\left(0^{+}\right)-h\left(0^{-}\right)\right]$, we can rewrite

$$
\Delta E_{k+1}-\Delta E_{k}=A\left(1-n_{0} \Delta z_{k}\right)
$$

The energy difference, $\Delta E_{k+1}-\Delta E_{k}$, depends linearly on the initial distance between the particles (Fig. 2.11).

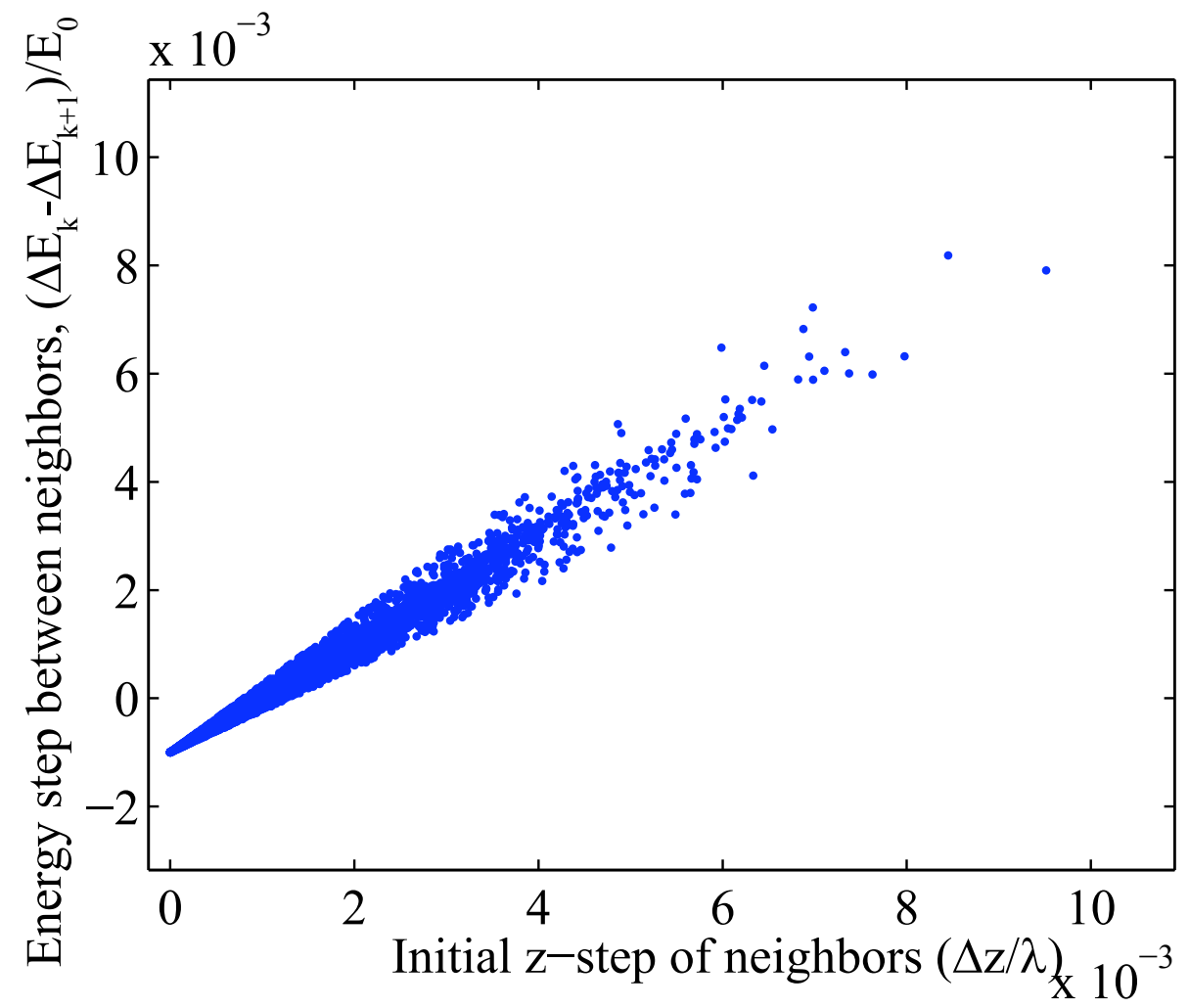

Figure 2.11: The relative modulation between neighboring particles, $E_{k+1}-E_{k}$, is proportional to the initial distance between the particles (sheets), $\Delta z$. Simulation is for the undulator interaction, with $\Upsilon=1, n_{0} \lambda_{0}=$ $10^{3}$. Particles that are closer (farther) than the inter-particle spacing, $\Delta z<1 / n_{0}$, lose (gain) energy relative to the previous particle, and move away (closer) in negative dispersion.

Following the dispersive section of strength $R_{56}$, the new distance between particles $k$ and $k+1$ is

$$
\begin{aligned}
\Delta z_{k}^{\text {new }} & =\Delta z_{k}+R_{56}\left(\Delta E_{k+1}-\Delta E_{k}\right) \\
& =R_{56} A+\left(1-R_{56} n_{0} A\right) \Delta z_{k} .
\end{aligned}
$$

At full suppression, $\Upsilon=R_{56} n_{0} A=1$, we obtain

$$
\Delta z_{k}^{\text {new }}=\frac{1}{n_{0}},
$$


giving a uniform structure with inter-particle spacing of $1 / n_{0}$, as in a quasi-crystalline beam [52].

The increase in noise at low frequencies (Eq. 2.31) leads to variation in local density, and thus variation in particle spacing. From simulations we confirm $\Delta z_{k}^{\text {new }}=1 / \bar{n}_{0}$, where $\bar{n}_{0}$ is the local density over a region of length $L_{n}$ with $1 / n_{0} \ll L_{n} \ll \lambda_{0} / N_{u}$. In reality, the beam is not transversely uniform as assumed in the 1D sheet model, so we do not expect a rigid quasi-crystalline structure. However, we still expect to find an amplification of the bunching factor at the inverse of the inter-particle spacing. Though the uniform beam is an intriguing theoretical result, due to the requirement of cold beam $\left(\sigma_{\eta} \ll 1 / n_{0} R_{56}\right)$ and a true step function interaction, practical applications may prove elusive.

\subsection{Effects of Energy Spread and Modulation}

In the earlier sections we treated only the case of vanishing energy spread, $\sigma_{\eta} \rightarrow 0$. When $k R_{56} \sigma_{\eta} \gtrsim 1$, the energy spread factor, $\exp \left[-k^{2} R_{56}^{2} \sigma_{\eta}^{2}\right]$, washes out noise suppression; at high enough frequencies, noise suppression fails. To suppress noise for larger $k$ we must decrease the dispersive strength, $R_{56}$. However, from $\Upsilon \equiv R_{56} n_{0} A=1$, weak dispersion implies a strong interaction, $A$, which in turn heats the beam. To determine an upper limit on the interaction strength, we consider how the energy modulation in the suppression process affects the energy spread of the beam.

\subsubsection{Energy Modulation from Noise Suppression}

The noise suppression process (interaction and dispersive regions) changes the particle energies, $\eta \rightarrow \hat{\eta}$ (Fig. 2.12). For the undulator case, the expected amplitude of the resulting energy modulation for a single particle is

$$
\begin{aligned}
\left\langle h_{u}^{2}(\zeta)\right\rangle & =\int_{0}^{N_{u} \lambda_{0}} \frac{d \zeta}{N_{u} \lambda_{0}} h_{u}^{2}(\zeta) \\
& =A_{u}^{2} \int_{0}^{1} d \bar{\zeta}(1-\bar{\zeta})^{2} \cos ^{2} \alpha \bar{\zeta} \\
& =\frac{A_{u}^{2}}{24}\left(4+\frac{6}{\alpha^{2}}-\frac{2 \sin 2 \alpha}{\alpha^{3}}\right) .
\end{aligned}
$$

With $\alpha \equiv 2 \pi N_{u} \geq 2 \pi$, we drop the final two terms. We can then guess that if a particle interacts with on average $\bar{N}=n_{0} N_{u} \lambda_{0}$ particles, the expected rms energy spread will be approximately

$$
\langle\Delta \eta\rangle \approx \sqrt{\bar{N}\left\langle h_{u}^{2}(\zeta)\right\rangle} \approx \sqrt{\frac{n_{0} N_{u} \lambda_{0}}{6}} A_{u} .
$$

Combined with the suppression condition, $\Upsilon=-n_{0} R_{56} A_{u} \sim 1$, we have

$$
\langle\Delta \eta\rangle \approx-\sqrt{\frac{N_{u} \lambda_{0}}{6 n_{0}}} \frac{1}{R_{56}}
$$

for the case of maximum suppression (Fig. 2.13). 


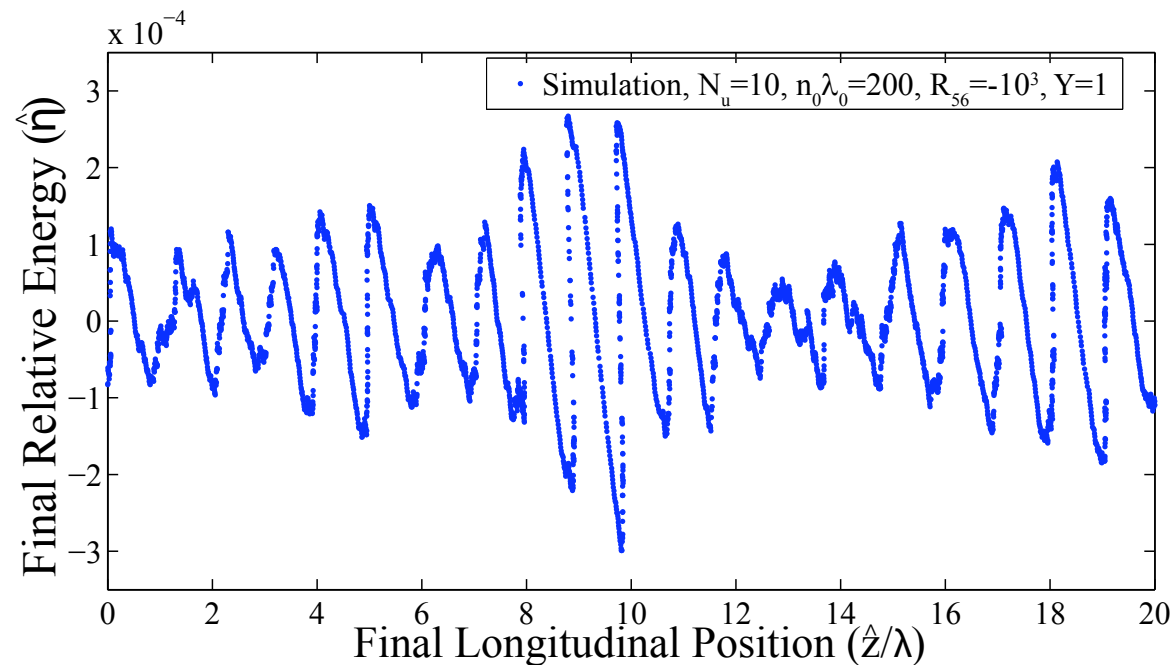

Figure 2.12: Phase space of the particles following both the interaction and dispersive regions. Example is for the undulator case with uniform initial energy, $\sigma_{\eta}=0$.

\subsubsection{Suppression Wavelength Limit}

The energy spread washes out noise suppression for wavelengths below $\lambda_{\min } \sim 2 \pi \sigma_{\eta} R_{56}$. Expressing $\lambda_{\min }$ in terms of the energy modulation, we find

$$
\lambda_{\min }=2 \pi \sqrt{\frac{N_{u} \lambda_{0}}{6 n_{0}}} \frac{\sigma_{\eta}}{\langle\Delta \eta\rangle} .
$$

From Eq. 2.51, we note that suppression is possible even when the modulation amplitude is small compared to the beam's natural energy spread, $\langle\Delta \eta\rangle \ll \sigma_{\eta}$ (Fig. 2.14). Decreasing $R_{56}$ extends suppression to shorter wavelengths, but heats the beam. If we require that the interaction has negligible effect on the energy spread, then we find a lower limit on $\lambda_{\min }$ when $\langle\Delta \eta\rangle \sim \sigma_{\eta}$.

\subsubsection{Energy Spread for FEL}

Our goal is to create a quiet beam, so we would like to consider the extent to which reducing shot noise will amplify energy noise. For example, FELs require energy spreads smaller than the Pierce parameter, $\rho$, giving $\langle\Delta \eta\rangle \lesssim 10^{-3}$ for current XFEL designs [11].

Quiet beams may be useful for controlling FEL start-up, which is driven by noise, $F(k)$, for SASE FELs, and from an external radiation field for seeded FELs. However, there is also a contribution to the FEL start up from the energy noise [53],

$$
F_{\eta}(k)=\frac{1}{N} \sum_{j, l} \frac{\hat{\eta}_{j} \hat{\eta}_{l}}{\rho^{2}} e^{i k\left[\hat{z}_{j}-\hat{z}_{l}\right]}
$$

We note that $F_{\eta}(k)$ scales as $\hat{\eta}^{2}$, which is always small. However, if $\hat{\eta}_{j}(z)$ is longitudinally periodic (as can be seen in Fig. 2.12 for $\left.k=k_{0}\right), F_{\eta}(k)$ will also scale as the number of particles, $N$, which is generally very large. To claim a quiet start up for an FEL, we must ensure that $F_{\eta}(k) \lesssim F(k)$. 


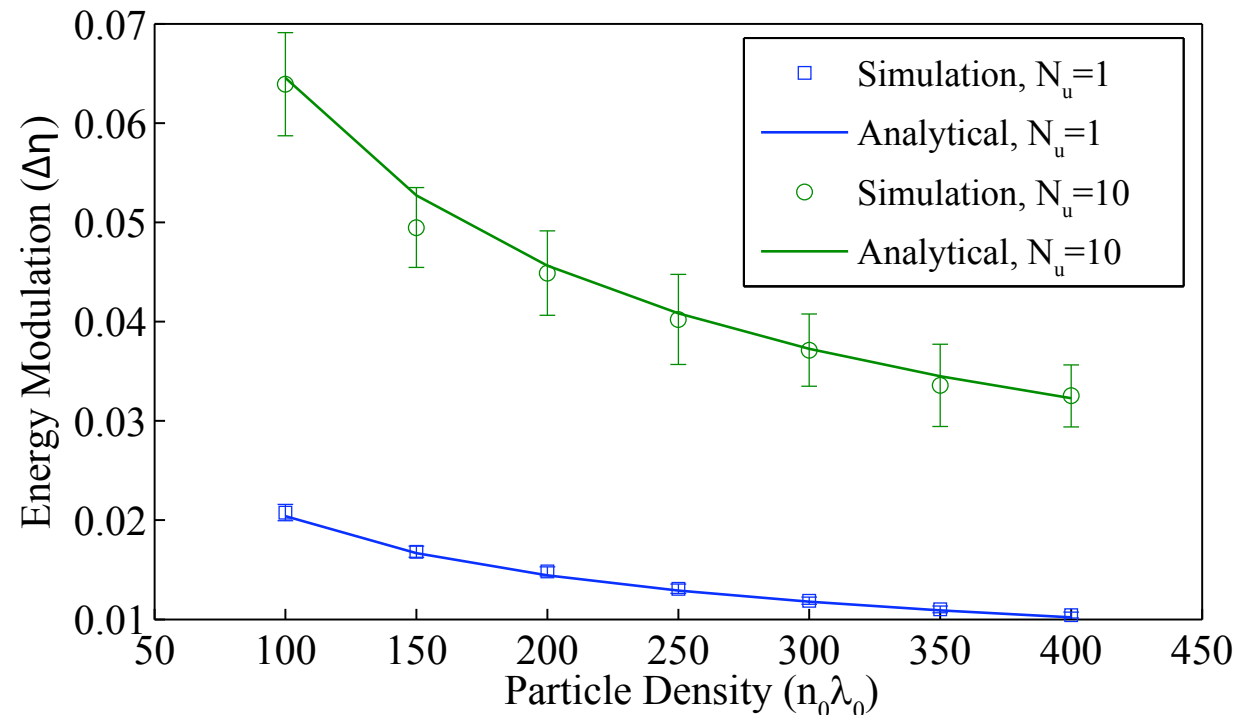

Figure 2.13: RMS energy modulation, $\langle\Delta \eta\rangle$, induced by the interaction (Eq. 2.50) is compared to the result of repeated simulations for the undulator case. The final energy spread is given as a function of the particle per wavelength, $n_{0} \lambda_{0}$, for $N_{u}=1$ and 10 , and initial energy spread $\sigma_{\eta}=0$.

\subsection{Extension to 3D Model}

The reader may wonder how accurately our 1D model matches 3D reality. In particular, Ref. [25] showed that in the high frequency limit $(a \gg \gamma \lambda)$ 3D longitudinal space charge fields deviate significantly from the 1D approximations. In this section we show that the longitudinal space charge fields are approximately equal in the 1D and 3D models at high frequency, but only after we include transverse averaging across the beam (see Eqs. 2.55 and 2.56 below). We also provide a fully 3D simulation showing that noise suppression occurs even at high frequencies in a 3D system (Fig. 2.16).

However, even if the 1D longitudinal fields are a sufficiently accurate approximation of reality, there are still differences between the 1D and 3D models. For example, the particles see slightly different longitudinal fields at the center and edge of the beam (Fig. 2.3). Moreover, in our rigid 1D disc, all particles move according to the average field seen across the whole disc. By contrast, in the 3D model, each particle moves independently of the particles around it. (This could be stated as a deformation of the rigid disc of the 1D model.) We then may guess that a 3D simulation will give weaker noise suppression, and indeed we see that Fig. 2.16 shows less noise suppression than its 1D equivalent (Fig. 2.6). This may correspond to analogous differences between 3D and 1D models in suppression through plasma oscillations [14, 15].

In the following sections we provide a detailed comparison of 1D vs. 3D models for the case of a space charge interaction. In the first portion we use the approach of Ref. [25] to show that 1D models provide an accurate representation of the average longitudinal space charge fields for a 3D system. Derivations of the equations in section 2.11.1 are given in appendix, chapter A. In the second portion, we provide results from a 3D simulation confirming that noise suppression is possible in a 3D system. 


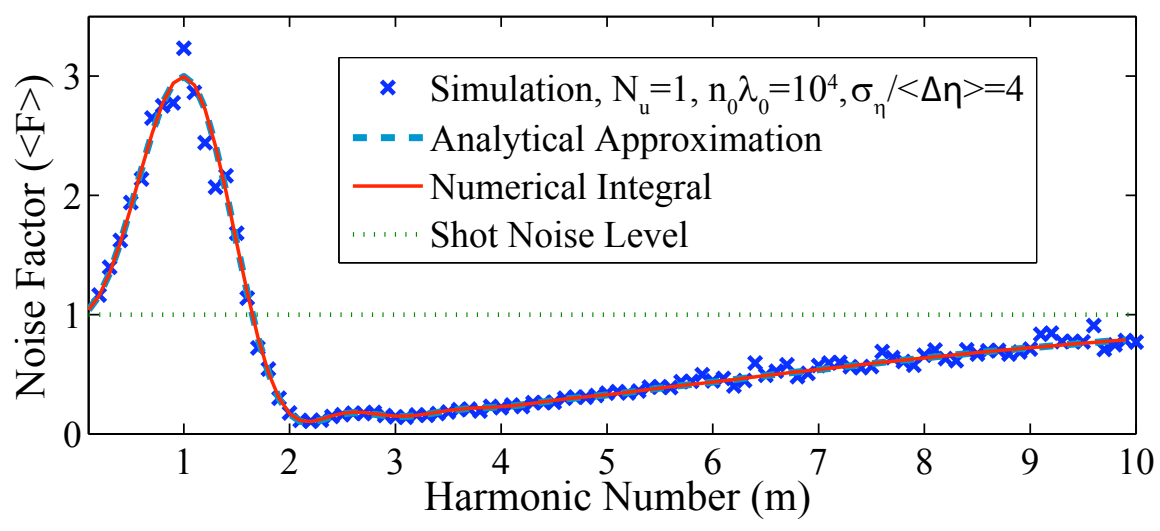

Figure 2.14: Noise suppression for the case $N_{u}=1$ with $\langle\Delta \eta\rangle \approx \sigma_{\eta} / 5$. For the parameters given, $\lambda_{\min } \approx$ $\lambda_{0} / 8$. With higher charge density, $n_{0}$, suppression extends to shorter wavelengths.

\subsubsection{Analytical Comparison of 1D and 3D models}

Venturini calculates the quantity $\left\langle E_{k}\left(r_{1}\right) E_{k}^{*}\left(r_{2}\right)\right\rangle$, which is proportional to our noise factor, $\langle F(k)\rangle$. First, we consider the 1D case. Evaluating at the center of the beam $\left(r_{1}=r_{2}=0\right)$, Venturini finds

$$
\left\langle\tilde{E}_{k} \tilde{E}_{k}^{*}\right\rangle_{1 D: r_{1}, r_{2}=0}=\left(\frac{e}{2 \pi^{2} \epsilon_{0} \gamma r_{b}}\right)^{2} N_{b}\left(\frac{1-\xi_{b} K_{1}\left(\xi_{b}\right)}{\xi_{b}}\right)^{2} .
$$

Venturini then repeats the calculation of $\left\langle E_{k}\left(r_{1}\right) E_{k}^{*}\left(r_{2}\right)\right\rangle$ at $r_{1}=r_{2}=0$ with a 3D model (particles of random transverse distribution) and finds (Eq. 11 of [25])

$$
\left\langle\tilde{E}_{k} \tilde{E}_{k}^{*}\right\rangle_{3 D: r_{1}, r_{2}=0}=\left(\frac{e}{2 \pi^{2} \epsilon_{0} \gamma r_{b}}\right)^{2} N_{b} \frac{1+\xi_{b}^{2}\left[K_{0}^{2}\left(\xi_{b}\right)-K_{1}^{2}\left(\xi_{b}\right)\right]}{4} .
$$

Venturini notes that the 1D and 3D models diverge in the high frequency limit, $a \gg \gamma \lambda$, as is evident in his Fig. 1.

However, while Venturini evaluates the quantity at $r_{1}=r_{2}=0$, we are interested in a transverse average over $r_{1}$ and $r_{2}$; for the noise factor we require the longitudinal field experienced by each particle due to every other particle in the bunch. (Summing over all particles in the calculation of $F(k)$ is equivalent to averaging $F(k)$ over the transverse extent of the beam.) We then need to calculate the transverse average $\int r_{1} d r_{1} \int r_{2} d r_{2}\left\langle E_{k}\left(r_{1}\right) E_{k}^{*}\left(r_{2}\right)\right\rangle$ rather than just evaluating at $r_{1}=r_{2}=0$. If we assume a flat-top transverse distribution (a uniform disc of charge) of radius $r_{b}$, we find the transversely averaged result

$$
\left\langle\tilde{E}_{k} \tilde{E}_{k}^{*}\right\rangle_{1 D \perp}=\left(\frac{e}{2 \pi^{2} \epsilon_{0} \gamma r_{b}}\right)^{2} N_{b}\left(\frac{1-2 K_{1}\left(\xi_{b}\right) I_{1}\left(\xi_{b}\right)}{\xi_{b}}\right)^{2}
$$

equivalent to the expression in Eq. 2.24, but also found elsewhere (e.g. Refs. [49, 50]).

We can then repeat this average for the 3D model. Again assuming a uniform transverse distribution function, but now with randomly distributed particles, we find (see appendix, chapter A)

$$
\left\langle\tilde{E}_{k} \tilde{E}_{k}^{*}\right\rangle_{3 D \perp}=\left(\frac{e}{2 \pi^{2} \epsilon_{0} \gamma r_{b}}\right)^{2} N_{b}\left[\left(\frac{1-2 K_{1}\left(\xi_{b}\right) I_{1}\left(\xi_{b}\right)}{\xi_{b}^{2}}\right)^{2}+\delta\right]
$$


where we have defined

$$
\delta(x) \equiv K_{1}\left(\xi_{b}\right)^{2}\left(\xi_{b} I_{1}\left(\xi_{b}\right) I_{2}\left(\xi_{b}\right)+\frac{\xi_{b}^{2}}{4}\left(I_{2}\left(\xi_{b}\right)^{2}-I_{1}\left(\xi_{b}\right)^{2}\right)\right)
$$

to rewrite the 3D result in the same form as the 1D result (Eq. A.2). Comparing Eqs. A.2 and A.4, we see that the 1D and 3D expressions are similar; the only difference is the $\delta$ term, which is generally small.

To better understand the comparison with the $r_{1}=r_{2}=0$ results, we also formulate a numerical integral that we can average for an arbitrary transverse portion of the beam. For this 3D result we follow Venturini's approach as an added check. We then numerically integrate over an arbitrary portion of the beam. So, for example, to integrate from 0 to some radius $r$, we evaluate (again, see appendix, chapter A)

$$
\begin{aligned}
\left\langle\tilde{E}_{k} \tilde{E}_{k}^{*}\right\rangle_{3 D \perp 0 \rightarrow r} & =\left(\frac{e}{2 \pi^{2} \epsilon_{0} \gamma r_{b}}\right)^{2} \frac{4 N_{b}}{\xi_{b}^{4}} \int_{0}^{r} d y \int_{y}^{r} d z z y \\
& {\left[K_{0}(y) K_{0}(z) \frac{y^{2}}{2}\left(I_{0}(y)^{2}-I_{1}(y)^{2}\right)\right.} \\
& +\frac{I_{0}(y) I_{0}(z)}{2}\left(\xi_{b}^{2} K_{0}\left(\xi_{b}\right)^{2}-\xi_{b}^{2} K_{1}\left(\xi_{b}\right)^{2}-z^{2} K_{0}(z)^{2}+z^{2} K_{1}(z)^{2}\right) \\
& \left.+\int_{y}^{z} d x x I_{0}(y) K_{0}(z) I_{0}(x) K_{0}(x)\right] .
\end{aligned}
$$

Note that by removing the $z, y$ integrals, and setting $y \rightarrow 0$, and $z \rightarrow \xi$, we recover Venturini's Eq. 12 .

We can now compare all of our 1D and 3D results (see Fig. 2.15 below). We confirm Venturini's result of a divergence between the 1D and 3D models for $\xi_{b}>1$ when $r_{1}=r_{2}=0$. However, for our noise factor, $\langle F(k)\rangle$, we need to calculate the average over the entire beam, and in that limit we find that the results are nearly identical for the 1D and 3D cases. Indeed, Venturini's Eq. 12 hints at this result; he calculates $\left\langle E_{k}(0) E_{k}^{*}(r)\right\rangle$, and finds that this quantity falls steeply to zero in the 3D limit when $k \sigma / \gamma \gg 1$ and $r \neq 0$ (See Fig. 2 of Ref. [25]). While the 3D result predicts stronger correlations when $r_{1}=r_{2}$ (Fig. 1 of Ref. [25]), it predicts weaker correlations when $r_{1} \neq r_{2}$ (Fig.2 of Ref. [25]), and the average is in fact similar to the 1D model. We then conclude that the 1D model is a good approximation of the 3D model in the high frequency limit.

(We note that the precise difference between the 1D and 3D averages depends on the transverse beam distribution. The results given here assume a disc of uniform expectation value. However, a Gaussian transverse distribution results in a difference of a factor of $4 / 3$, as found in Chapter 3 in Section 3.6.2.)

\subsubsection{D Simulation}

As the most direct test of the model we can simply carry out a 3D simulation. We modify our 1D code to calculate the standard coulomb interaction between particles distributed in a longitudinally uniform and transversely flat-top beam. The 3D code is severely limited by computational constraints; because we must calculate the interaction between all particles, the number of operations scales as the square of the particle number, and the 3D simulations require many more particles than the 1D simulations. However, we are able to simulate approximately $10^{4}$ particles in a length of $L=a$. Despite the computational constraints, we still observe noise suppression at low frequencies (Fig. 2.16). (At high frequencies there are too few particles per wavelength for an accurate calculation.) We see that the noise suppression improves as we increase the number of particles in the simulation. 


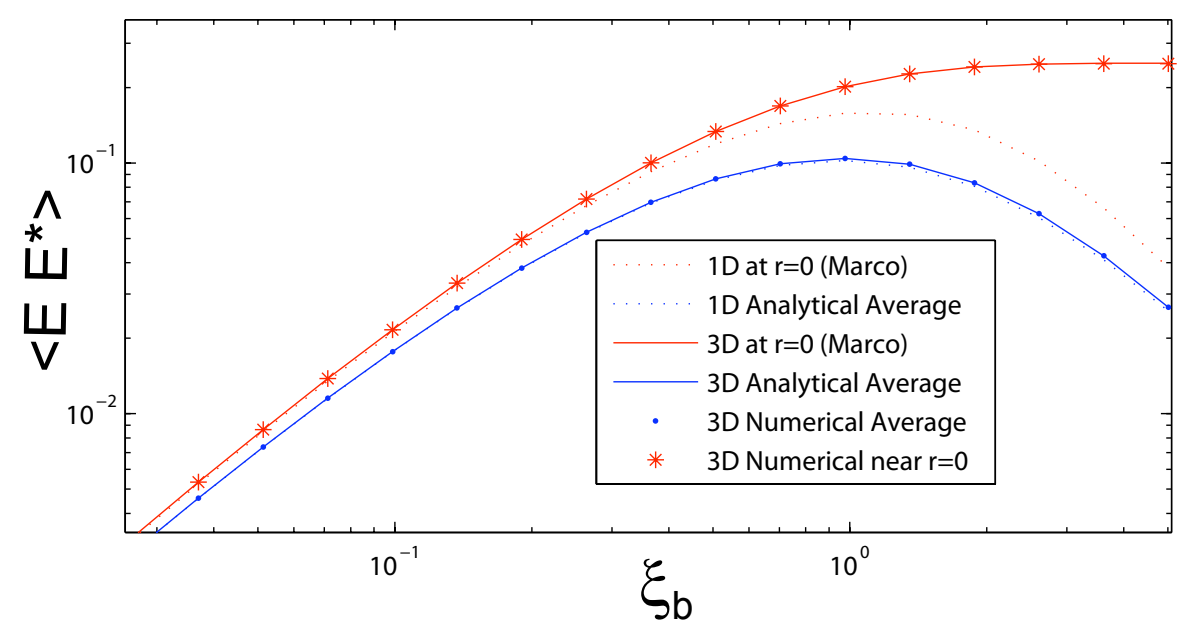

Figure 2.15: Comparison of 1D and 3D models for both $r=0$ (red curves) and transverse average (blue). When evaluated at $r=0$, the 1D and 3D models diverge at high frequencies $\left(\xi_{b} \gg 1\right)$, as shown by Venturini. However, when averaged transversely, the 1D and 3D results are nearly identical even at high frequency. The numerical integral, which matches Venturini's Eq. 12 and reproduces the $r_{1}=r_{2}=0$ case, also confirms our analytical result. These results are for a transverse flat-top distribution. See Fig. 3.6 for the equivalent results for a Gaussian transverse distribution.

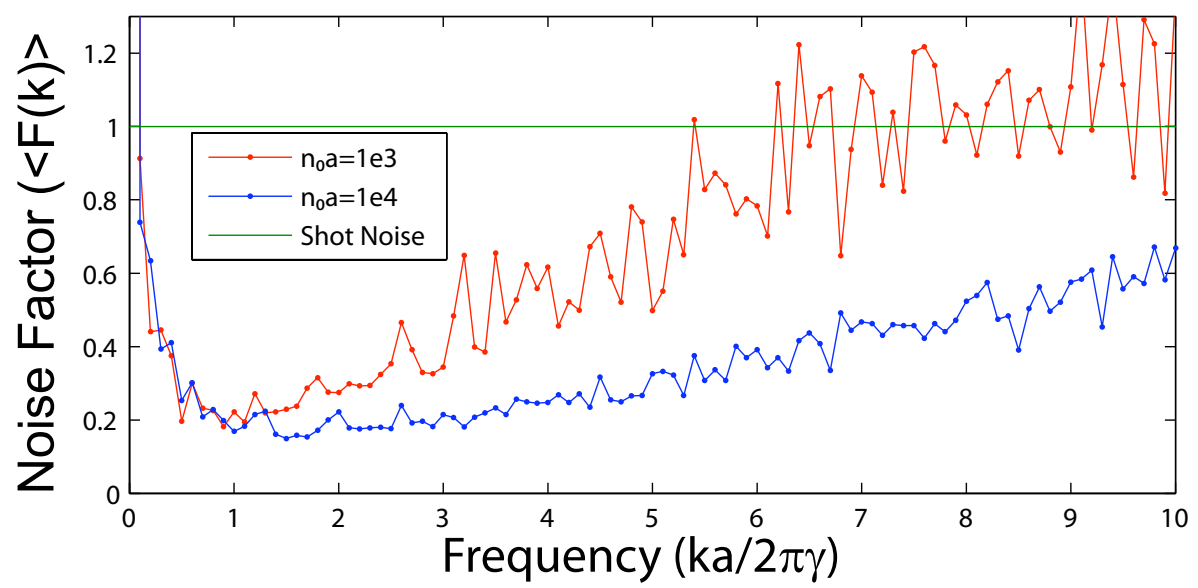

Figure 2.16: Noise suppression for the 3D space charge case. The suppression starts to fail when the number of particles is too low, so our simulations improve with larger particle number. Ideally we would simulate higher particle densities, but are limited by computational times. 


\section{Chapter 3}

\section{D Shot Noise Model of Microbunching}

\subsection{Introduction}

In a high-brightness linear accelerator, as used for a high-gain free electron laser (FEL), impedance effects along the accelerator can drive a periodic microbunching instability that degrades the electron beam qualities $[6,18,19,20,21,22,23,24]$. Longitudinal space charge (LSC) forces may dominate microbunching gain at wavelengths shorter than the electron bunch [21, 22, 23, 24]. LCLS has observed evidence of optical wavelength microbunching from optical transition radiation (OTR) screens. In particular, OTR screens downstream from the high-brightness injector have recorded Coherent OTR (COTR) from an uncompressed electron bunch [1]. At such short modulation wavelengths (less than $1 \mu \mathrm{m}$ ), electron shot noise is the most probable source of the initial density fluctuations required to start the instability.

Venturini noted [25] that the purely longitudinal model of LSC impedance used in previous studies [21, $22,23,24]$ may fail at very short wavelengths, when the longitudinal shot noise modulations are not transversely uniform. In this paper, we present a six-dimensional (6D) analysis of LSC microbunching starting from shot noise. Using a simplified machine model motivated by the LCLS setup, we calculate the microbunching gain factor for short modulation wavelengths observed at small angles relative to the longitudinal direction. We also compare these results with the LCLS COTR observations [26]. The results of this chapter were first presented as Ref. [46].

\subsection{Model Assumptions}

Our goal is to calculate microbunching derived from the electric fields of randomly distributed electrons. To derive the bunching behavior, we start with a random distribution of electrons in full 6D phase space. The unevenly distributed electrons produce LSC fields, which in turn cause energy modulations to accumulate along the accelerator. A dispersive element with nonzero momentum compaction can then convert the energy modulation to change in longitudinal position (Fig. 3.1). As a result, the longitudinal density distribution is modified from the initial random distribution and may show enhancement at some frequency range, i.e., the beam is microbunched at these frequencies.

In practice, we observe microbunching as COTR emitted from thin foils in the beam path. The radiated 


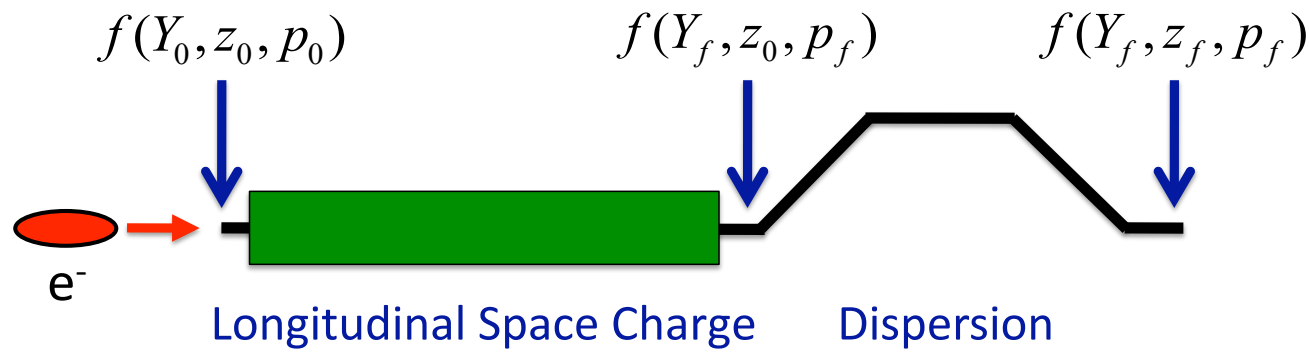

Figure 3.1: Schematic of the interaction-dispersion model system. Longitudinal space charge causes an energy modulation, which then produces a density modulation following a dispersive region. The dispersion may be positive or negative.

COTR has power given by

$$
\left(\frac{d^{2} I}{d \omega d \Omega}\right)_{\text {tot }}=\left(\frac{d^{2} I}{d \omega d \Omega}\right)_{1}|b(\boldsymbol{k})|^{2}
$$

with the single-electron angular spectrum

$$
\left(\frac{d^{2} I}{d \omega d \Omega}\right)_{1} \propto \frac{\gamma^{4}\left(\theta_{x}^{2}+\theta_{y}^{2}\right)}{\left[1+\gamma^{2}\left(\theta_{x}^{2}+\theta_{y}^{2}\right)\right]^{2}} .
$$

We will quantify the degree of bunching at position $L$ in the accelerator by the bunching factor

$$
b(\boldsymbol{k})=\frac{1}{N} \sum_{j} \exp \left[-i \tilde{K} X_{j}(L)\right] \exp \left[-i k \delta z_{j}(L)\right],
$$

with bunching wave vector

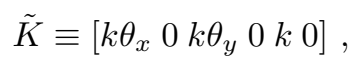

electron coordinates

$$
\boldsymbol{X}=\left[\begin{array}{c}
x \\
x^{\prime} \\
y \\
y^{\prime} \\
z \\
p
\end{array}\right]
$$

and longitudinal deviation from space charge

$$
\delta z_{j} \equiv \int_{0}^{L} d s \frac{e}{\gamma_{s} m c^{2}} R_{s \rightarrow L}^{(56)} E_{z}\left(R_{0 \rightarrow s} X_{0 j}\right) .
$$

In these definitions a tilde denotes transpose, $E_{z}$ is the electric field experienced by the $j$ th electron at position $s$ in the accelerator, and the sum is over all electrons in the bunch. The final COTR scales as $|b(\boldsymbol{k})|^{2}$, so our task will be to find the expectation value $\left\langle|b(\boldsymbol{k})|^{2}\right\rangle$. Note that the bunching factor normalization here is different from that given in Eq. 1.1, with $b(k) \equiv f(k) / \sqrt{N}$. 


\subsection{Longitudinal Space Charge Field}

Longitudinal space charge (LSC) forces derived from the electron bunch shot noise produce microbunching. We are interested in the high-frequency microbunching regime, $k \sigma_{T} / \gamma \gg 1$, when the transverse coherence length is smaller than the transverse beam size, $\sigma_{T}$. Because the coherence length is only captured in a full 6D model of microbunching, we derive our LSC field from a 6D shot noise distribution, i.e., a sum of 6D delta functions.

$$
\rho(\boldsymbol{X})=\sum_{j}^{N} \delta\left(x-x_{j}\right) \delta\left(y-y_{j}\right) \delta\left(z-z_{j}\right) \delta\left(x^{\prime}-x_{j}^{\prime}\right) \delta\left(y^{\prime}-y_{j}^{\prime}\right) \delta\left(p-p_{j}\right) .
$$

We assume the only space charge effect of interest is the energy modulation of the electrons by the LSC electric fields

$$
\begin{aligned}
E_{z}\left(\boldsymbol{X}_{1}\right) & =\frac{e}{4 \pi \epsilon_{0}} \int d \boldsymbol{X}_{2} G\left(\boldsymbol{X}_{1}, \boldsymbol{X}_{2}\right) \rho\left(\boldsymbol{X}_{2}\right) \\
G\left(\boldsymbol{X}_{1}, \boldsymbol{X}_{2}\right) & \equiv \frac{\left(z_{1}-z_{2}\right) \gamma}{\left[\left(x_{1}-x_{2}\right)^{2}+\left(y_{1}-y_{2}\right)^{2}+\gamma^{2}\left(z_{1}-z_{2}\right)^{2}\right]^{3 / 2}},
\end{aligned}
$$

with electron energy, $\gamma$. From Eq. 3.7 and the 6D distribution, Eq. 3.6, we find the longitudinal E-field

$$
E_{j}(\boldsymbol{X}(s))=\frac{e}{4 \pi \epsilon_{0}} \sum_{i}^{N} \frac{\left[z_{j}(s)-z_{i}(s)\right] \gamma}{\left(\left[x_{j}(s)-x_{i}(s)\right]^{2}+\left[y_{j}(s)-y_{i}(s)\right]^{2}+\gamma^{2}\left[z_{j}(s)-z_{i}(s)\right]^{2}\right)^{3 / 2}}
$$

with electron coordinate $\boldsymbol{X}(s)$ a function of accelerator position. Eq. 3.7 depends only on the instantaneous particle positions, $x, y$ and $z$, but we want to integrate the E-field all along the accelerator, and the general particle positions $x(s), y(s)$, and $z(s)$ depend on the full initial phase space, $x_{0}, y_{0}, z_{0}, x_{0}^{\prime}, y_{0}^{\prime}, p_{0}$. As a result, we must keep the full $6 \mathrm{D}$ distribution.

The instantaneous LSC field affects the final $z$ position of the $j$-th particle according to

$$
\frac{d \delta z_{j}(s)}{d s}=\frac{R_{s \rightarrow L}^{(56)} e E\left(X_{j}(s)\right)}{\gamma_{s} m c^{2}} .
$$

We now further assume the space charge effect is weak enough that we need only carry it to first order in all relevant quantities. Though we allow $\delta z_{j}(s)$ to vary along the accelerator, we will ignore any changes to the LSC field from the microbunching process. We will designate $\boldsymbol{X}_{0 j}$ as the initial coordinates of the $j$-th particle. The particle phase space coordinates, $\boldsymbol{X}_{j}(s)$, are determined only by the transfer matrix, $R_{0 \rightarrow s}$, the initial coordinates, $\boldsymbol{X}_{0 j}$, and the resulting space charge induced energy modulation, which we keep only to first order. The final coordinates of the $j$ th particle at the observation point, $L$, are then given by

$$
X_{j}^{f} \approx R_{0 \rightarrow L} X_{0 j}+\delta z_{j}
$$

with

$$
\delta z_{j} \equiv \frac{e}{m c^{2}} \int_{0}^{L} d s\left[\begin{array}{c}
0 \\
0 \\
0 \\
0 \\
0 \\
\frac{R_{s \rightarrow L}^{(56)} E_{z}}{\gamma_{s}}\left(R_{0 \rightarrow s} X_{0 j}\right)
\end{array}\right]
$$


with electron energy at position $s$ of $\gamma_{s} m c^{2}$, and impedance section of length $L$.

To facilitate the bunching calculation, we will use the longitudinal Fourier transform of our 6D shot noise E-field, Eq. 3.8, which is of the form

$$
\int d k e^{-i k z} \frac{\gamma z}{\left(|Y|^{2}+\gamma^{2} z^{2}\right)^{3 / 2}}=\frac{-2 i k}{\gamma^{2}} K_{0}\left(\frac{k|Y|}{\gamma}\right),
$$

where we have defined $|Y|$ as the length of transverse coordinates $\boldsymbol{Y}$ both for brevity and also to emphasize that the Bessel function depends only on particle positions, not angles.

Finally, in this LSC model we've made two assumptions that are violated in the ultra-relativistic regime. First, we assume all particles travel in the $z$ direction, so that the electric fields are not tilted with respect to the motion of the electron bunch. This assumption holds so long as the angular motion is small, $x^{\prime}, y^{\prime} \ll 1 / \gamma$. Second, we have determined the energy change to a particle at accelerator position (or time) $s$ by calculating the E-field from the other particle coordinates at the same time, $s$, rather than using the retarded time, $s_{\text {ret }}$. This assumption holds if the catch-up distance $1 / k(1-\beta) \approx 2 \gamma^{2} / k$ is small compared to the electron motion (e.g. the $\beta$ function, waist size, etc.). (Note, for $\sigma_{T} \gg \gamma / k$, we should replace the wavelength with the transverse size in the previous relation.)

\subsection{Expectation Value of Bunching Factor Squared}

\subsubsection{Weak Modulation Expansion}

The main task at hand is the evaluation of the expectation value of the bunching factor squared,

$$
\left\langle|b(\boldsymbol{k})|^{2}\right\rangle=\frac{1}{N}\left\langle\sum_{j}^{N} \sum_{l}^{N} e^{-i \tilde{K}\left(X_{j}(L)-X_{l}(L)\right)} e^{-i k\left(\sum_{i \neq j} \delta z_{j i}-\sum_{i \neq l} \delta z_{l i}\right)}\right\rangle,
$$

with expression for the energy modulation effect

$$
\sum_{i \neq j}^{N} \delta z_{j, i}=\sum_{i \neq j}^{N} \frac{e}{m c^{2}} \frac{e}{4 \pi \epsilon_{0}} \int d s \frac{R_{s \rightarrow L}^{(56)}}{\gamma_{s}} \frac{\partial}{\partial z_{i}} \frac{1}{\left|X_{j}(s)-X_{i}(s)\right|} .
$$

To find the expectation value, we start by separating the $j=l$ and $j \neq l$ cases. We will start with the incoherent portion, $j=l$. In setting the two indices equal we lose one summation, and we can see that the phase terms all go to unity. The result is simply the shot noise of an electron beam

$$
\left\langle|b(\boldsymbol{k})|^{2}\right\rangle_{S N}=\frac{1}{N} \sum_{j}^{N} 1=1 .
$$

Next we have the coherent portion, with $j \neq l$. To evaluate the expectation value, we integrate over the final particle distribution function, $\Psi_{N}\left(X_{1}(L), \ldots, X_{N}(L)\right)$. However, $\Psi_{N}$ may be a complicated function of the $N$ variables and the beam transfer matrices, $R$. Instead, we will rewrite our bunching factor in terms of the initial coordinates through $X(s)=R_{0 \rightarrow s} X_{0}$, and then integrate over the simpler initial distributions, $\Psi_{N}\left(X_{01}, \ldots X_{0 N}\right)$. In particular, we assume the initial beam is uncorrelated (random) so that we can decompose

$$
\Psi_{N}\left(X_{01}, \ldots, X_{0 N}\right)=\prod_{i}^{N} \Psi\left(X_{0 i}\right)
$$


with the single particle distribution functions, $\Psi$. (We blindly trust our laser experts who tell us the beam must - MUST! - be longitudinally structureless coming off the cathode.) We assume all particles are identical, so the sums over $j$ and $l$ each give a factor of $N$, and we can integrate over the single particle distributions to find

$$
\left\langle|b(\boldsymbol{k})|^{2}\right\rangle_{C} \equiv N \int d X_{01} \ldots d X_{0 N} \Psi\left(X_{01}\right) \ldots \Psi\left(X_{0 N}\right) e^{-i \tilde{K}\left(R_{0 \rightarrow L} X_{01}-R_{0 \rightarrow L} X_{02}\right)} e^{-i k\left(\sum_{i \neq 1} \delta z_{1 i}-\sum_{i \neq 2} \delta z_{2 i}\right)}
$$

where we have assigned $j=1, l=2$ without loss of generality and approximated $N-1 \approx N$. Following Stupakov [17], our approach is to explicitly isolate the portions of the integrand which do not contain any dependence on $i \neq 1,2$,

$$
\begin{aligned}
\left\langle|b(\boldsymbol{k})|^{2}\right\rangle_{C}= & N \int d X_{01} \ldots d X_{0 N} \Psi\left(X_{01}\right) \ldots \Psi\left(X_{0 N}\right) e^{-i \tilde{K}\left(R_{0 \rightarrow L} X_{01}-R_{0 \rightarrow L} X_{02}\right)} e^{-i k\left(\delta z_{1,2}-\delta z_{2,1}\right)} \\
& e^{-i k \sum_{i=3}^{N}\left(\delta z_{1, i}-\delta z_{2, i}\right)} \\
= & N \int d X_{01} \int d X_{02} \Psi\left(X_{01}\right) \Psi\left(X_{02}\right) e^{-i \tilde{K}\left(R_{0 \rightarrow L} X_{01}-R_{0 \rightarrow L} X_{02}\right)} e^{-i k\left(\delta z_{1,2}-\delta z_{2,1}\right)} \\
& {\left[\int d X_{0 i} \Psi\left(X_{0 i}\right) e^{-i k\left(\delta z_{1, i}-\delta z_{2, i}\right)}\right]^{(N-2)} }
\end{aligned}
$$

where we've again used the assumption of identical particles to convert the sum into a product of $N-2$ identical integrals. To facilitate the calculation of the expectation value, we will assume the longitudinal motion resulting from the modulation is small $(|k \delta| \ll 1)$, so that we can linearize the exponents

$$
\left\langle|b(\boldsymbol{k})|^{2}\right\rangle_{C}=N \int d X_{01} \int d X_{02} \Psi\left(X_{01}\right) \Psi\left(X_{02}\right) e^{-i \tilde{K}\left(R_{0 \rightarrow L} X_{01}-R_{0 \rightarrow L} X_{02}\right)}\left[1+\Gamma_{1}\right]\left[1+\Gamma_{2} / N\right]^{N-2} .
$$

with definitions expanded in powers of $k \delta$

$$
\begin{aligned}
\Gamma_{1} & \equiv-i k\left(\delta z_{1,2}-\delta z_{2,1}\right)-k^{2}\left(\delta z_{1,2}-\delta z_{2,1}\right)^{2}+\ldots \\
\Gamma_{2} & \equiv N \int d X_{0 i} \Psi\left(X_{0 i}\right)\left(-i k\left(\delta z_{1, i}-\delta z_{2, i}\right)-\frac{1}{2}\left[k\left(\delta z_{1, i}-\delta z_{2, i}\right)\right]^{2}+\ldots\right) .
\end{aligned}
$$

Because $\delta$ is small, we will keep only the lowest order terms. We will assume that the interaction length, $L_{\text {int }}$, of the space charge is small compared to the bunch length, $L$. (That is, for $L \gg\left|X_{j}-X_{i}\right|>L_{\text {int }}$, we find $\delta z_{i j} \rightarrow 0$. This assumption also lets us ignore edge effects.) We then find $\left\langle\delta z_{1, i}\right\rangle=\left\langle\delta z_{2, j}\right\rangle$ and the linear order terms of $\Gamma_{2}$ integrate to 0 . Then keeping the lowest order non-zero terms for $\Gamma_{1}$ (linear in $k \delta$ ) and $\Gamma_{2}$ (quadratic in $k \delta$ ) gives

$$
\begin{aligned}
& \Gamma_{1} \approx-i k\left(\delta z_{1,2}-\delta z_{2,1}\right) \\
& \Gamma_{2} \approx-\frac{N k^{2}}{2} \int d X_{0 i} \Psi\left(X_{0 i}\right)\left[\left(\delta z_{1, i}-\delta z_{2, i}\right)\right]^{2} .
\end{aligned}
$$

Finally, when we plug back into Eq. 3.19, we will have a term with an integral over the phases $\exp \left[i k X_{01}\right]$ and $\exp \left[i k X_{02}\right]$ multiplied by $\Gamma_{2}$. Unless $\Gamma_{2}$ cancels both phase factors, the integral must average to zero. Hence the $\delta z_{1, i}^{2}$ and $\delta z_{2, i}^{2}$ terms (with no dependence on $X_{02}$ and $X_{01}$ respectively) must vanish for $k \neq 0$, and we drop them from $\Gamma_{2}$. (Strictly speaking, these factors vanish only for $k>1 / L$, but we are not interested in the 
case $k<1 / L$ anyway.) In the end we have two competing coherent terms:

$$
\begin{aligned}
& \Gamma_{1} \approx-i k\left(\delta z_{1,2}-\delta z_{2,1}\right) \\
& \Gamma_{2} \approx N k^{2} \int d X_{0 i} \Psi\left(X_{0 i}\right) \delta z_{1, i} \delta z_{2, i} .
\end{aligned}
$$

The first term $\left(\Gamma_{1}\right.$, henceforth the linear term) is linear in $k \delta$, while the second term $\left(\Gamma_{2}\right.$, henceforth the quadratic term) is quadratic in $k \delta$. While $k \delta$ is presumed to be small, we cannot ignore the quadratic term because it also contains a factor of $N$, which can be a billion or larger for the case of LCLS. We will instead evaluate both terms, and decide from the particular machine parameters which term will dominate.

\subsubsection{Initial Distribution}

To carry out the integrals, we will need to specify the initial distribution, $\Psi\left(X_{0}\right)$. We will assume a decoupled distribution $\Psi\left(X_{0}\right)=\Psi\left(Y_{0}\right) \Psi\left(z_{0}\right) \Psi\left(p_{0}\right)$, with $Y_{0}$ containing all transverse coordinates. We can then write

$$
\Psi\left(X_{0}\right)=\frac{1}{(2 \pi)^{3} \sqrt{\operatorname{det} U}} \exp \left[-\frac{1}{2} \tilde{X}_{0} U^{-1} X_{0}\right]
$$

where $U$ is the beam's second-moment matrix (symmetric, positive definite) at initial position $s=0$,

$$
U=\left[\begin{array}{cccccc}
\left\langle x^{2}\right\rangle & \left\langle x x^{\prime}\right\rangle & 0 & 0 & 0 & 0 \\
\left\langle x x^{\prime}\right\rangle & \left\langle x^{\prime 2}\right\rangle & 0 & 0 & 0 & 0 \\
0 & 0 & \left\langle y^{2}\right\rangle & \left\langle y y^{\prime}\right\rangle & 0 & 0 \\
0 & 0 & \left\langle y y^{\prime}\right\rangle & \left\langle y^{\prime 2}\right\rangle & 0 & 0 \\
0 & 0 & 0 & 0 & \left\langle z^{2}\right\rangle & 0 \\
0 & 0 & 0 & 0 & 0 & \left\langle p^{2}\right\rangle
\end{array}\right]
$$

Though we start with a decoupled distribution, at any arbitrary position along the accelerator we will find the longitudinal and transverse components mix together. In particular, the gaussian transverse distribution will include a $z$ component. If we assume the bunch is long compared to the wavelengths of interest, we can drop the $z^{2}$ term and separate out the $z$ components. However, coupling between longitudinal and transverse components implies a dispersive section, possibly violating our constraint of carrying the microbunching modulation only to first order.

Instead, we will concentrate on the case of a dispersion-less interaction region followed by a single interaction-free dispersive section (Fig. 2.1). We then have no longitudinal motion during the modulation process and the longitudinal distribution decouples from the transverse distribution throughout the interaction. If we further assume the bunch length is long relative to the wavelength of interest, we can approximate the longitudinal distribution as a flat-top

$$
\Psi(z)=\left\{\begin{array}{ll}
1 / L, & |z| \leq \sigma_{z} \\
0, & |z|>\sigma_{z}
\end{array} .\right.
$$

We will assume a flat-top distribution for the rest of the chapter. 


\subsubsection{Weak LSC Limit}

In the Section 3.4.1 we expanded the exponentials in the limit $k \delta \ll 1$. If we further assume $\Gamma_{2} \ll 1$ we can expand again to find

$$
\left\langle|b(\boldsymbol{k})|^{2}\right\rangle_{C}=N \int d X_{01} \int d X_{02} \Psi\left(X_{01}\right) \Psi\left(X_{02}\right) e^{-i \tilde{K}\left(R_{0 \rightarrow L} X_{01}-R_{0 \rightarrow L} X_{02}\right)}\left[\Gamma_{1}+\Gamma_{2}\right]+f(k<1 / L) .
$$

with the same definitions

$$
\begin{aligned}
& \Gamma_{1} \approx-i k\left(\delta z_{1,2}-\delta z_{2,1}\right) \\
& \Gamma_{2} \approx N k^{2} \int d X_{0 i} \Psi\left(X_{0 i}\right) \delta z_{1, i} \delta z_{2, i} .
\end{aligned}
$$

The function $f(k<1 / L)$ comes from integrating over the phase factor for the leading (constant) term in the argument $1+\Gamma_{1}+\Gamma_{2}+\ldots$. We are interested in wavelengths shorter than the bunch (i.e., microbunching), so we will drop $f(k<1 / L)$.

In the following sections we will evaluate the linear and quadratic terms individually. We also note that it is in principle possible to continue without the weak limit approximation. Using instead $\left[1+\Gamma_{2} / N\right]^{N} \approx$ $\exp \left[\Gamma_{2}\right]$, we may still solve for the expectation value either analytically or numerically. We give an example of such a solution in a 1D case for shot noise suppression (Chap. 2). While this approach may be necessary to check simulations with small numbers of particles and thus large interaction strengths $\delta z$, for most practical cases the weak limit approximation is valid.

\subsubsection{Evaluating the Quadratic Term}

\section{Three Transformations}

We are particularly interested in the quadratic term, which drives the microbunching instability. Pulling out the quadratic term $\left(\Gamma_{2}\right)$ from Eq. 3.26, we can write down the increase in bunching at the final accelerator position, $s=L$,

$$
\left\langle|b(\boldsymbol{k})|^{2}\right\rangle_{\left(\delta z^{2}\right)} \equiv N^{2} \int d X_{01} \int d X_{02} \Psi\left(X_{01}\right) \Psi\left(X_{02}\right) e^{-i \tilde{K}\left(R_{0 \rightarrow L} X_{01}-R_{0 \rightarrow L} X_{02}\right)} \Gamma_{2}\left(X_{01}, X_{02}\right) .
$$

As in Section 3.4.1, we've written the expression in terms of the initial coordinates, $X_{0} \equiv R_{L \rightarrow 0} X(L)$, the first of three variable transformations we will make. It is now time to plug in for our particular $\delta$ in the case of space charge

$$
\begin{aligned}
\left\langle|b(\boldsymbol{k})|^{2}\right\rangle_{\left(\delta z^{2}\right)} & =(N-1)^{2} k^{2} \int d X_{01} \Psi\left(X_{01}\right) \int d X_{02} \Psi\left(X_{02}\right) \int d X_{0 i} \Psi\left(X_{0 i}\right) \\
& e^{-i \tilde{K}\left(R_{0 \rightarrow L} X_{01}-R_{0 \rightarrow L} X_{02}\right)}\left(\frac{e^{2}}{4 \pi \epsilon_{0} m c^{2}}\right)^{2} \\
& \int d s_{1} \frac{R_{s_{1} \rightarrow L}^{(56)}}{\gamma_{1}} \frac{\partial}{\partial z_{0 i}} \frac{1}{\left|X_{01}\left(s_{1}\right)-X_{0 i}\left(s_{1}\right)\right|} \int d s_{2} \frac{R_{s_{2} \rightarrow L}^{(56)}}{\gamma_{2}} \frac{\partial}{\partial z_{0 i}} \frac{1}{\left|X_{02}\left(s_{2}\right)-X_{0 i}\left(s_{2}\right)\right|}
\end{aligned}
$$

The potential depends on only the difference between the two particle coordinates, so we can simplify the expression by changing to difference variables, the second of our three transformations: 


$$
\xi_{01,02}=X_{0 i}-X_{01,02}
$$

This transformation allows us to separate out the $X_{0 i}$ integrals

$$
\begin{aligned}
\left\langle|b(\boldsymbol{k})|^{2}\right\rangle_{\left(\delta z^{2}\right)} & =N^{2} k^{2} \int d \xi_{02} \int d \xi_{01} e^{-i \tilde{K} R_{0 \rightarrow L}\left(\xi_{02}-\xi_{01}\right)}\left(\frac{e^{2}}{4 \pi \epsilon_{0} m c^{2}}\right)^{2} \\
& \int d s_{1} \frac{R_{s_{1} \rightarrow L}^{(56)}}{\gamma_{1}} \frac{\partial}{\partial z_{01}} \frac{1}{\left|R_{0 \rightarrow s_{1}} \xi_{01}\right|} \int d s_{2} \frac{R_{s_{2} \rightarrow L}^{(56)}}{\gamma_{2}} \frac{\partial}{\partial z_{02}} \frac{1}{\left|R_{0 \rightarrow s_{2}} \xi_{02}\right|} \\
& \int d X_{0 i} \Psi\left(X_{0 i}\right) \Psi\left(X_{0 i}-\xi_{01}\right) \Psi\left(X_{0 i}-\xi_{02}\right) .
\end{aligned}
$$

The Coulomb field (Eq. 3.8) depends only on the particle positions $(x, y, z)$, and not on the angles $\left(x^{\prime}, y^{\prime}, z^{\prime}\right)$; at any given moment, the interaction dependence spans only half of the $6 \mathrm{D}$ phase space. However, at present we have the field written in terms of the initial coordinates, $X_{0}$, and the interaction will in general depend on all six initial coordinates. To simplify, we transform variables a third time to a changing reference frame

$$
\xi_{1,2} \equiv R_{0 \rightarrow s_{1,2}} \xi_{01,02}
$$

(We call it changing because it varies as a function of longitudinal position in the accelerator, s.) In the changing basis we have

$$
\begin{gathered}
\left\langle|b(\boldsymbol{k})|^{2}\right\rangle_{\left(\delta z^{2}\right)}=N^{2} k^{2}\left(\frac{e^{2}}{4 \pi \epsilon_{0} m c^{2}}\right)^{2} \int d s_{1} \int d s_{2} \int \frac{d \xi_{2}}{J_{2}} \int \frac{d \xi_{1}}{J_{1}} e^{-i \tilde{K} R_{s \rightarrow L}\left(\xi_{2}-\xi_{1}\right)} \frac{R_{s_{1} \rightarrow L}^{(56)}}{\gamma_{1}} \frac{R_{s_{2} \rightarrow L}^{(56)}}{\gamma_{2}} \\
\frac{\partial}{\partial z_{1}} \frac{1}{\left|\xi_{1}\right|} \frac{\partial}{\partial z_{2}} \frac{1}{\left|\xi_{2}\right|} \int d X_{0 i} \Psi\left(X_{0 i}\right) \Psi\left(X_{0 i}-R_{s_{1} \rightarrow 0} \xi_{1}\right) \Psi\left(X_{0 i}-R_{s_{2} \rightarrow 0} \xi_{2}\right)
\end{gathered}
$$

with $J$ the Jacobian of the transformation $R_{0 \rightarrow s}$.

\section{Twenty Integrals}

Eq. 3.33 contains twenty integrals. Our strategy will be to separate out the E-field and accelerator position dependence $\left(x_{1}, y_{1}, x_{2}, y_{2}, s_{1}, s_{2}\right)$, and evaluate the remaining integrals first.

Taking the assumption of a long bunch $(L \gg 1 / k)$, we use a flat-top distribution (Eq. 3.25). The $z$ components of the $\xi_{1,2}$ integrations then become Fourier transforms, giving

$$
\begin{aligned}
\left\langle|b(\boldsymbol{k})|^{2}\right\rangle_{\left(\delta z^{2}\right)} & =n_{0}^{2}\left(\frac{e^{2} k^{2}}{2 \pi \epsilon_{0} m c^{2}}\right)^{2} \int d s_{1} \int d s_{2} \int \frac{d \bar{\xi}_{2}}{J_{2}} \int \frac{d \bar{\xi}_{1}}{J_{1}} \frac{R_{s_{1} \rightarrow L}^{(56)}}{\gamma_{1}^{3}} \frac{R_{s_{2} \rightarrow L}^{(56)}}{\gamma_{2}^{3}} \\
& e^{-i \tilde{K} R_{s \rightarrow L}\left(\bar{\xi}_{2}-\bar{\xi}_{1}\right)} e^{-i k\left[R_{s \rightarrow L}^{(51)}\left(x_{2}-x_{1}\right)+R_{s \rightarrow L}^{(52)}\left(x_{2}^{\prime}-x_{1}^{\prime}\right)+R_{s \rightarrow L}^{(56)}\left(p_{2}-p_{1}\right)\right]} \\
& K_{0}\left(\left|Y_{1}\right| \frac{k}{\gamma_{1}}\right) K_{0}\left(\left|Y_{2}\right| \frac{k}{\gamma_{2}}\right) \int d \bar{X}_{0 i} \Psi\left(\bar{X}_{0 i}\right) \Psi\left(\bar{X}_{0 i}-R_{s_{1} \rightarrow 0} \bar{\xi}_{1}\right) \Psi\left(\bar{X}_{0 i}-R_{s_{2} \rightarrow 0} \bar{\xi}_{2}\right),
\end{aligned}
$$

with longitudinal density, $n_{0} \equiv N / L$, and where we've used $Y$ to denote transverse coordinates, and barred variables, $\bar{\xi}, \bar{X}$, to denote all $\xi, X$ components except for $z$ (i.e., $Y$ plus $p$ ). We note here that two factors of $\gamma_{1} \gamma_{2} \equiv \gamma\left(s_{1}\right) \gamma\left(s_{2}\right)$ come from the E-field, but one factor comes from the normalization of $R_{s \rightarrow L}^{(56)}$, which is defined in terms of relative energy; in practice, it may be desirable to take $R_{s \rightarrow L}^{(56)}$ from an arbitrary accelerator coordinate, $s$, even perhaps outside the integration range, but the energy factor must change accordingly. 
Carrying out the $p_{1}, p_{2}$ integrals, we find

$$
\begin{aligned}
\left\langle|b(\boldsymbol{k})|^{2}\right\rangle_{\left(\delta z^{2}\right)} & =n_{0}^{2}\left(\frac{e^{2} k^{2}}{2 \pi \epsilon_{0} m c^{2}}\right)^{2} e^{-k^{2} R_{56}^{2} p_{0}^{2}} \int d s_{1} \int d s_{2} \int \frac{d Y_{2}}{J_{2}} \int \frac{d Y_{1}}{J_{1}} \frac{R_{s_{1} \rightarrow L}^{(56)}}{\gamma_{1}^{3}} \frac{R_{s_{2} \rightarrow L}^{(56)}}{\gamma_{2}^{3}} \\
& e^{-i \tilde{K} R_{s \rightarrow L}\left(Y_{2}-Y_{1}\right)} \\
& K_{0}\left(\left|Y_{1}\right| \frac{k}{\gamma_{1}}\right) K_{0}\left(\left|Y_{2}\right| \frac{k}{\gamma_{2}}\right) \int d Y_{0 i} \Psi\left(Y_{0 i}\right) \Psi\left(Y_{0 i}-R_{s_{1} \rightarrow 0} Y_{1}\right) \Psi\left(Y_{0 i}-R_{s_{2} \rightarrow 0} Y_{2}\right),
\end{aligned}
$$

and are left with just transverse components $(Y)$.

Having chosen a transverse phase space distribution (Eq.3.23), we are in position to start evaluating the remaining fourteen integrals in Eq.3.35. To start, we can write down the Gaussian $Y_{0 i}$ integral, which is of the form

$$
\begin{aligned}
I_{G}= & \int d V \Psi\left(V-V_{1}\right) \Psi\left(V-V_{2}\right) \Psi(V) \\
& =\frac{\left(\operatorname{det} U^{-1}\right)^{3 / 2}}{(2 \pi)^{6}} \int d V \exp \left[-\frac{1}{2}\left(\tilde{V}-\tilde{V}_{1}\right) U^{-1}\left(V-V_{1}\right)-\frac{1}{2}\left(\tilde{V}-\tilde{V}_{2}\right) U^{-1}\left(V-V_{2}\right)-\frac{1}{2} \tilde{V} U^{-1} V\right] .
\end{aligned}
$$

Making the change of variables $W \equiv \sqrt{3} V-\left(V_{1}+V_{2}\right) / \sqrt{3}$, we can rewrite the integral as

$$
I_{G}=\frac{\left(\operatorname{det} U^{-1}\right)^{3 / 2}}{(2 \pi)^{6}} \int \frac{d W}{9} \exp \left[-\frac{1}{2} \tilde{W} U^{-1} W-\frac{1}{3}\left(\tilde{V}_{1} U^{-1} V_{1}+\tilde{V}_{2} U^{-1} V_{2}-\tilde{V}_{1} U^{-1} V_{2}\right)\right] .
$$

Carrying out the integral over $W$, and plugging in for $V_{1,2}=R_{s_{1,2} \rightarrow 0} Y_{1,2}$, we find the Gaussian integral

$$
\begin{aligned}
& I_{G}\left(Y_{1}, Y_{2}\right)= \\
& \quad \frac{\operatorname{det} U^{-1}}{9(2 \pi)^{4}} \exp \left[-\frac{1}{3}\left(\tilde{Y}_{1} \tilde{R}_{s_{1} \rightarrow 0}-\tilde{Y}_{2} \tilde{R}_{s_{2} \rightarrow 0}\right) U^{-1}\left(R_{s_{1} \rightarrow 0} Y_{1}-R_{s_{2} \rightarrow 0} Y_{2}\right)-\frac{1}{3} \tilde{Y}_{1} \tilde{R}_{s_{1} \rightarrow 0} U^{-1} R_{s_{2} \rightarrow 0} Y_{2}\right] .
\end{aligned}
$$

We still have ten integrals $\left(s_{1,2} x_{1,2}, y_{1,2}, x_{1,2}^{\prime}, y_{1,2}^{\prime}\right)$ remaining in Eq.3.35. The goal of our third transformation (to a changing basis) was to remove the angular dependence in the Bessel functions. We can then pull these factors out of the angle integrals to find

$$
\begin{aligned}
\left\langle|b(\boldsymbol{k})|^{2}\right\rangle_{\left(\delta z^{2}\right)} & =n_{0}^{2}\left(\frac{e^{2} k^{2}}{2 \pi \epsilon_{0} m c^{2}}\right)^{2} \frac{\operatorname{det} U^{-1}}{9(2 \pi)^{4}} e^{-k^{2} R_{56}^{2} p_{0}^{2}} \int d s_{1} \int d s_{2} \frac{R_{s_{1} \rightarrow L}^{(56)}}{\gamma_{1}^{3}} \frac{R_{s_{2} \rightarrow L}^{(56)}}{\gamma_{2}^{3}} \\
& \int \frac{r_{1} d r_{1} d \theta_{1}}{J_{1}} \int \frac{r_{2} d r_{2} d \theta_{2}}{J_{2}} K_{0}\left(\frac{r_{1} k}{\gamma_{1}}\right) K_{0}\left(\frac{r_{2} k}{\gamma_{2}}\right) e^{-i k R_{s \rightarrow L}^{(5)}\left(r_{2} \cos \theta_{2}-r_{1} \cos \theta_{1}\right)} G_{2},
\end{aligned}
$$

with polar coordinate, $r$, defined as the length of vector $Y$, and Gaussian integral over angles

$$
\begin{aligned}
G_{2} & \equiv \int d x_{1}^{\prime} d y_{1}^{\prime} \int d x_{2}^{\prime} d y_{2}^{\prime} e^{-i \tilde{K}\left[R_{s_{2} \rightarrow L} Y_{2}-R_{s_{1} \rightarrow L} Y_{1}\right]} e^{-i k R_{s \rightarrow L}^{(52)}\left(x_{2}^{\prime}-x_{1}^{\prime}\right)} \\
& \exp \frac{1}{3}\left[\tilde{Y}_{1} \tilde{R}_{s_{1} \rightarrow 0} U^{-1} R_{s_{2} \rightarrow 0} Y_{2}-\left(\tilde{Y}_{1} \tilde{R}_{s_{1} \rightarrow 0}-\tilde{Y}_{2} \tilde{R}_{s_{2} \rightarrow 0}\right) U^{-1}\left(R_{s_{1} \rightarrow 0} Y_{1}-R_{s_{2} \rightarrow 0} Y_{2}\right)\right]
\end{aligned}
$$

where $\mathrm{J}$ is the Jacobian of the transformation $R_{0 \rightarrow s}$ and we've explicitly written out the $R_{51}$ and $R_{52}$ dependence (which would otherwise be hidden in the $R Y$ terms). Though it looks intimidating, $G_{2}$ is just a 
Gaussian integral which we can evaluate exactly after specifying the transverse motion $R_{s \rightarrow 0}$ and $R_{s \rightarrow L}$. After evaluating $G_{2}$, we are left with only six integrals, $r_{1,2}, \theta_{1,2}$ and $s_{1,2}$. We will see later it is often possible to evaluate the $r_{1,2}$ and $\theta_{1,2}$ integrals analytically in certain limits, leaving just two $s_{1,2}$ to be integrated numerically.

\subsubsection{Evaluating Linear Term Expectation Value}

We now return to the linear term,

$$
\left\langle|b(\boldsymbol{k})|^{2}\right\rangle_{(\delta z)} \equiv-N \int d X_{1} \int d X_{2} \Psi\left(X_{1}\right) \Psi\left(X_{2}\right) e^{-i \tilde{K}\left(X_{1}(L)-R_{0 \rightarrow L} X_{2}(L)\right)} \Gamma_{1}\left(X_{1}, X_{2}\right) .
$$

Following the same approach as for the quadratic term, we begin by rewriting the bunching factor in terms of initial coordinates,

$$
\begin{gathered}
\left\langle|b(\boldsymbol{k})|^{2}\right\rangle_{(\delta z)}=-2 N \int d X_{01} \int d X_{02} \Psi\left(X_{01}\right) \Psi\left(X_{02}\right) e^{-i \tilde{K}\left(R_{0 \rightarrow L} X_{01}-R_{0 \rightarrow L} X_{02}\right)} i k \\
\frac{e}{m c^{2}} \frac{e}{4 \pi \epsilon_{0}} \int d s \frac{R_{s \rightarrow L}^{(56)}}{\gamma_{s}} \frac{\partial}{\partial z_{2}} \frac{1}{\left|R_{0 \rightarrow s_{1}} X_{01}-R_{0 \rightarrow s_{1}} X_{02}\right|},
\end{gathered}
$$

where we've used the odd symmetry of Eq. 3.8 to combine the two terms in $\Gamma_{1}$. We then change to difference variable, $\xi_{01} \equiv X_{02}-X_{01}$, to find

$$
\begin{gathered}
\left\langle|b(\boldsymbol{k})|^{2}\right\rangle_{(\delta z)}=-2 N \int d \xi_{01} \int d X_{02} \Psi\left(X_{02}\right) \Psi\left(X_{02}-\xi_{01}\right) e^{i \tilde{K}\left(R_{0 \rightarrow L} \xi_{01}\right)} i k \\
\frac{e}{m c^{2}} \frac{e}{4 \pi \epsilon_{0}} \int d s \frac{R_{s \rightarrow L}^{(56)}}{\gamma_{s}} \frac{\partial}{\partial z_{1}} \frac{1}{\left|R_{0 \rightarrow s_{1}} \xi_{01}\right|},
\end{gathered}
$$

where we've swapped the derivative from $X_{02} \rightarrow \xi_{01}$ (using our new difference coordinate $z_{2}-z_{1}$ ). Switching the integration variable to $\xi_{1} \equiv R_{0 \rightarrow s_{1}} \xi_{01}$ we find

$$
\begin{gathered}
\left\langle|b(\boldsymbol{k})|^{2}\right\rangle_{(\delta z)}=-2 N \frac{i e k}{m c^{2}} \frac{e}{4 \pi \epsilon_{0}} \int d s \frac{R_{s \rightarrow L}^{(56)}}{J_{1} \gamma_{s}} \int d \xi_{1} e^{i \tilde{K}\left(R_{s \rightarrow L} \xi_{1}\right)} \frac{\partial}{\partial z_{1}} \frac{1}{\left|\xi_{1}\right|} \\
\int d X_{02} \Psi\left(X_{02}\right) \Psi\left(X_{02}-R_{s \rightarrow 0} \xi_{1}\right),
\end{gathered}
$$

with $J$ the Jacobian of the transformation $R_{0 \rightarrow s}$.

Assuming a longitudinally frozen beam we can separate out the transverse $(Y)$ and longitudinal $(z, p)$ components of the coordinates $\xi, X$. Then integrating over $p$ and $z$ (the latter a Fourier transform) we find

$$
\begin{gathered}
\left\langle|b(\boldsymbol{k})|^{2}\right\rangle_{(\delta z)}=-2 n_{0} R_{56} \frac{e k^{2}}{m c^{2}} \frac{e}{2 \pi \epsilon_{0}} e^{-k^{2} R_{56}^{2} \sigma_{p}^{2}} \int \frac{d s}{J_{1} \gamma^{3}} \int d Y_{1} e^{i \tilde{K}\left(R_{s \rightarrow L} Y_{1}\right)} e^{i k R_{51} x_{1}} e^{i k R_{52} x_{1}^{\prime}} K_{0}\left(\left|Y_{1}\right| \frac{k}{\gamma}\right) \\
\int d Y_{02} \Psi\left(Y_{02}\right) \Psi\left(Y_{02}-R_{s \rightarrow 0} Y_{1}\right),
\end{gathered}
$$

where we've plugged in Eq. 3.12. Rearranging factors we find

$$
\left\langle|b(\boldsymbol{k})|^{2}\right\rangle_{(\delta z)}=-2 n_{0} R_{56} k^{2} e^{-k^{2} R_{56}^{2} \sigma_{p}^{2}} \frac{2 I}{I_{A}} \int \frac{d s}{\gamma^{3}} \int \frac{r_{1} d r_{1} d \theta_{1}}{J_{1}} K_{0}\left(\frac{r_{1} k}{\gamma}\right) e^{i k R_{51} r_{1} \cos \theta_{1}} G_{1},
$$

with current $I=e c n_{0}$, Alfvén current $I_{A}=e c / r_{e}=4 \pi \epsilon_{0} m c^{3} / e$, and Gaussian integral

$$
\begin{aligned}
G_{1} & \equiv \int d x_{1}^{\prime} d y_{1}^{\prime} \int d Y_{0 j} e^{i \tilde{K}\left(R_{s \rightarrow L} Y_{1}\right)} e^{i k R_{52} x_{1}^{\prime}} \Psi\left(Y_{02}\right) \Psi\left(Y_{02}-R_{s \rightarrow 0} Y_{1}\right) \\
& =\frac{1}{4(2 \pi)^{2} \sqrt{\operatorname{det} U}} \int d x_{1}^{\prime} d y_{1}^{\prime} e^{i \tilde{K}\left(R_{s \rightarrow L} Y_{1}\right)} e^{i k R_{52} x_{1}^{\prime}} \exp \left[-\frac{1}{4} \tilde{Y}_{1} \tilde{R}_{s \rightarrow 0} U^{-1} R_{s \rightarrow 0} Y_{1}\right] .
\end{aligned}
$$


As in the quadratic case, $G_{1}$ is a Gaussian integral that can be evaluated numerically if the transfer matrices are known. The final expression, Eq. 3.46, then has three remaining integrals. We can now compare the relative amplitudes of our linear term, Eq. 3.46, with the quadratic term, Eq. 3.39.

\subsection{Results}

\subsubsection{Quadratic Term: Short Impedance Section}

To evaluate the full bunching for a practical case, we will look at a simplified model that is satisfied by the LCLS COTR observations. For this case we need only the quadratic term, Eq. 3.39, which dominates for $k^{2}|\delta z|^{2} \gg 1 / N$. (The linear term, Eq. 3.46, is just a small correction.)

To evaluate $G_{2}$ (Eq. 3.40), we need to choose the transverse particle motion in the accelerator. In principle we may evaluate Eq. 3.40 for any arbitrary accelerator motion:betatron oscillations with acceleration, laminar flow, drift, etc. Here we present only the simplest case of a short impedance section of total length $L \ll \beta$. We assume the impedance section is sufficiently short that we may approximate the beam as transversely frozen, and drop the $s$ integration. For the transfer matrices we then have $R_{0 \rightarrow s}=I$ and $R_{s \rightarrow l}=R_{0 \rightarrow l} \equiv R$ determined by the optics downstream of the impedance section. We focus on the spatial components of the final coordinates, $X_{j}$

$$
\begin{aligned}
& x_{j}=R_{11} x_{0}+R_{12} x_{0}^{\prime} \\
& y_{j}=R_{33} y_{0}+R_{34} y_{0}^{\prime} \\
& z_{j}=z_{0}+R_{51} x_{0}+R_{52} x_{0}^{\prime}+R_{56} p_{m},
\end{aligned}
$$

assuming no coupling between $x$ and $y$ to match the LCLS case. We then find

$$
\begin{aligned}
\tilde{K} R X= & k\left[z_{0}+R_{56} p_{0}+R_{1} x_{0}\right. \\
& \left.+R_{2} x_{0}^{\prime}+\theta_{y} R_{33} y_{0}+\theta_{y} R_{34} y_{0}^{\prime}\right] \\
R_{1} \equiv & R_{51}+\theta_{x} R_{11} \\
R_{2} \equiv & R_{52}+\theta_{x} R_{12} .
\end{aligned}
$$

Finally, to simplify the algebra we assume a circular beam $\left(\sigma_{x}=\sigma_{y}=\sigma\right)$. We can now carry out the angle integration, $G_{2}$, and switching to polar coordinates for the position variables, $x, y \rightarrow r, \phi$, we find

$$
\begin{aligned}
\left\langle|b(\boldsymbol{k})|^{2}\right\rangle_{\left(\delta z^{2}\right)} & =\left(\frac{e^{2} k^{2} R_{56} n_{0} L}{2 \pi \epsilon_{0} m c^{2} \gamma^{3}}\right)^{2} \frac{\operatorname{det} U^{-1} \sigma^{\prime 4}}{3(2 \pi)^{2}} \exp \left[-\sigma^{\prime 2} k^{2}\left(R_{2}^{2}+\theta_{y}^{2} R_{34}^{2}\right)\right] \\
& \int d r_{1} d r_{2} \exp \left[-\frac{r_{1}^{2}+r_{2}^{2}}{\sigma^{2}}\right] r_{1} K_{0}\left(\frac{k r_{1}}{\gamma}\right) r_{2} K_{0}\left(\frac{k r_{2}}{\gamma}\right) \\
& \int d \phi_{1} d \phi_{2} \exp \left[\frac{r_{1} r_{2} \cos \left(\phi_{1}-\phi_{2}\right)}{\sigma^{2}}\right] \\
& \exp \left[i k R_{1}\left(r_{1} \cos \phi_{1}-r_{2} \cos \phi_{2}\right)\right] \exp \left[i k \theta_{y} R_{33}\left(r_{1} \sin \phi_{1}-r_{2} \sin \phi_{2}\right)\right] .
\end{aligned}
$$

Defining $R_{q} \equiv \sqrt{R_{1}^{2}+\theta_{y}^{2} R_{33}^{2}}$ and $\tan \nu \equiv \theta_{y} R_{33} / R_{1}$ so that $R_{1} \cos \phi+\theta_{y} R_{33} \phi=R_{q} \cos (\phi+\nu)$, we can use $e^{i x \cos (\phi)}=\sum_{l} J_{l}(x) e^{i l(\phi+\pi / 2)}$ to move the $\phi$ dependence out of the exponent. Then, integrating over $\phi$ 
gives

$$
\begin{aligned}
\left\langle|b(\boldsymbol{k})|^{2}\right\rangle_{\left(\delta z^{2}\right)} & =\frac{1}{3}\left(\frac{e^{2} k^{2} R_{56} n_{0} L}{2 \pi \epsilon_{0} m c^{2} \gamma^{3} \sigma^{2}}\right)^{2} e^{\left[-\sigma^{\prime 2} k^{2}\left(R_{2}^{2}+\theta_{y}^{2} R_{34}^{2}\right)\right]} \\
& \int d r_{1} d r_{2} \exp \left[-\frac{r_{1}^{2}+r_{2}^{2}}{\sigma^{2}}\right] r_{1} K_{0}\left(\frac{k r_{1}}{\gamma_{1}}\right) r_{2} K_{0}\left(\frac{k r_{2}}{\gamma_{2}}\right) \\
& \sum_{l} I_{l}\left(\frac{-r_{1} r_{2}}{\sigma^{2}}\right) J_{l}\left(k r_{1} R_{q}\right) J_{-l}\left(-k r_{2} R_{q}\right) e^{-k^{2} R_{56}^{2} \sigma_{p}^{2}} .
\end{aligned}
$$

In the limit that $k \sigma / \gamma \gg 1$, we need only integrate out to $r \approx \gamma / k$, because $K_{0}\left(k r_{1} / \gamma\right)$ decays exponentially for large $r$. In this regime $(r \leq \gamma / k)$, the argument $r_{1} r_{2} / \sigma^{2}$ is always small and only the $I_{0} \approx 1$ term is non-negligible. Setting $l=0$, the $r_{1}$ and $r_{2}$ integrals separate, and we can use

$$
\begin{aligned}
\int_{0}^{\infty} x K_{0}(x) J_{0}(a x) & =\left.\frac{x}{a^{2}+1}\left[a J_{1}(a x) K_{0}(x)-J_{0}(a x) K_{1}(x)\right]\right|_{0} ^{\infty} \\
& =\frac{1}{a^{2}+1}
\end{aligned}
$$

to find expectation value of bunching

$$
\left\langle|b(\boldsymbol{k})|^{2}\right\rangle_{\left(\delta z^{2}\right)} \approx \frac{4}{3}\left[\frac{I}{I_{A} \gamma} \frac{R_{56} L}{\sigma^{2}}\right]^{2} \frac{e^{\left[-\sigma^{\prime 2} k^{2}\left(R_{2}^{2}+\theta_{y}^{2} R_{34}^{2}\right)-k^{2} R_{56}^{2} \sigma_{p}^{2}\right]}}{\left[\gamma^{2} R_{q}^{2}+1\right]^{2}},
$$

with average current $I_{0}=e c n_{0}$, and Alfvén current $I_{A}=e c / r_{e}=4 \pi \epsilon_{0} m c^{3} / e$ kA. If we include $s$ dependence (for instance from a drift section, or beta-oscillation), then the position $\left(R_{51}, R_{11}, R_{53}\right)$ and angle $\left(R_{52}, R_{12}, R_{43}\right)$ factors mix in both the suppression terms.

\subsection{LCLS Results}

The LCLS COTR observations in the absence of compression were initially described in Ref. [1] (see Fig. 44 and the paragraph above it). A schematic of the LCLS beamline through the first bunch compressor is given in Fig. 3.2. Following the initial observations of Ref. [1], a transmission grating spectrometer was installed in OTR12 in order to study COTR spectral content, and more data were collected in 2008 at $250 \mathrm{pC}$ bunch charge [26]. The integrated OTR signal maximizes at the QB quadrupole setting that makes the DL1 bend system a perfect linear achromat (see Fig. 3.3). When QB is set off peak, the nonzero $R_{51}$ and $R_{52}$ wash out the microbunching effect.

During this study, the spectral data were also collected at $\mathrm{QB}=10.7 \mathrm{kG}$ (peak COTR intensity) and at $\mathrm{QB}=11 \mathrm{kG}$ (baseline incoherent level). Analyzing the spectral data and taking the ratio of the two spectra, we obtain the OTR intensity gain as a function of the optical wavelength, shown in Fig. 3.5. We then use the experimental beam parameters to determine the theoretical gain curve. We take $\gamma_{0} m c^{2}=135 \mathrm{MeV}, \gamma_{0} \epsilon=1$ $\mu \mathrm{m}, \beta_{0}=1.2 \mathrm{~m}$, and the DL1 $R_{56}=6.3 \mathrm{~mm}$. We assume the waist in front of the dog leg dominates the impedance effects due to the small beta function there, so we take $L_{d} \approx 2.5 \mathrm{~m}$ for the beam waist extending $\pm 2 \mathrm{~m}$ from the waist at OTR2 (Fig. 3.4). The electron peak current is $I_{0} \approx 40 \mathrm{~A}$ for a $250 \mathrm{pC}$ electron

bunch with an rms length of $750 \mu \mathrm{m}$. Using these parameters, $k \sigma_{x_{0}} / \gamma_{0} \geq 2$ for $\lambda \leq 1 \mu \mathrm{m}$, and hence the high-frequency approximation is reasonable. We calculate the spectral gain by plugging Eq. 3.53 into

$$
\frac{d W}{d \omega}=\int_{-\theta_{m} / 2}^{\theta_{m} / 2} d \theta_{x} \int_{-\theta_{m} / 2}^{\theta_{m} / 2} d \theta_{y}\left(\frac{d^{2} W}{d \omega d \Omega}\right)_{1}\left[N+N^{2}|b(\boldsymbol{k})|^{2}\right]
$$




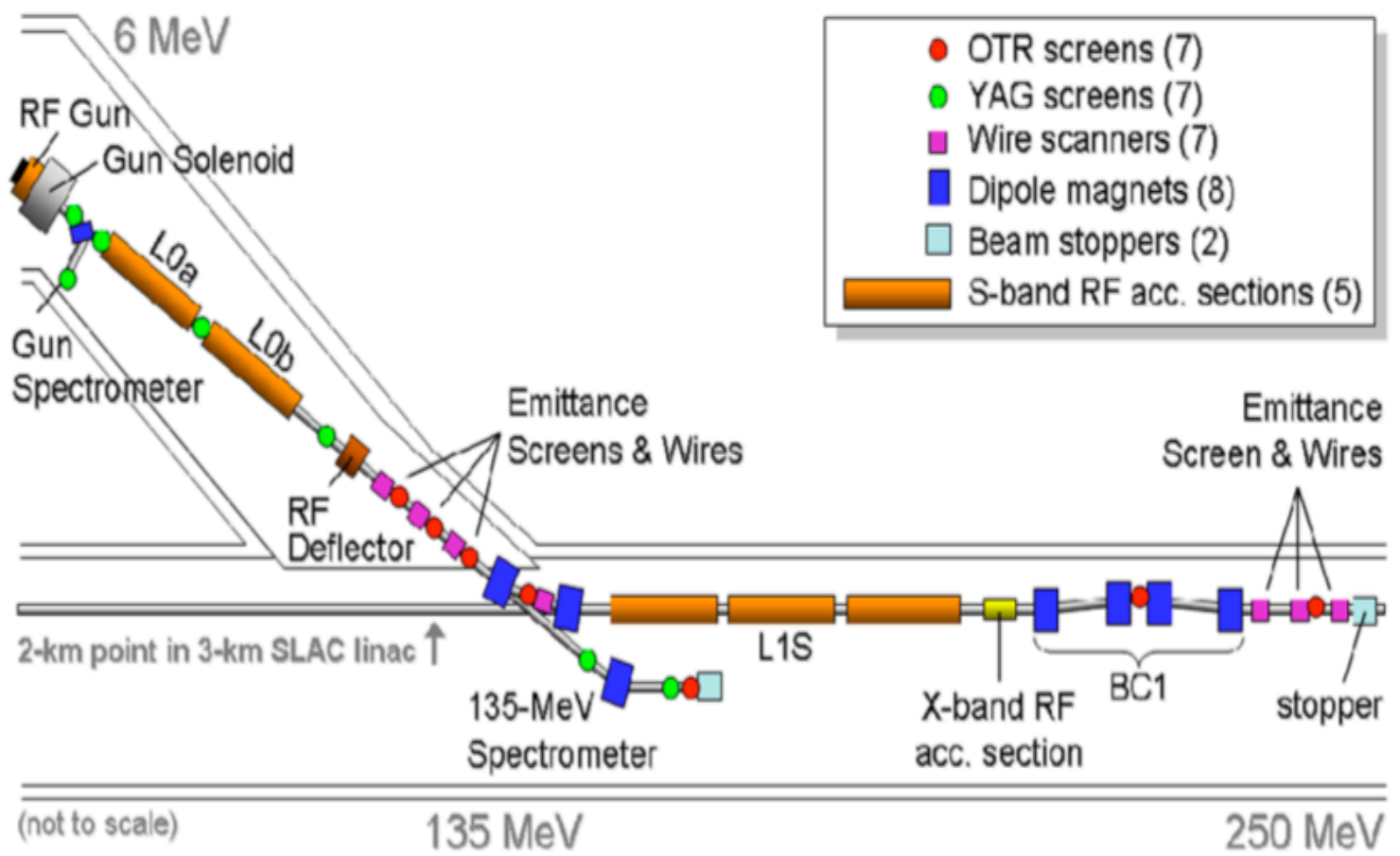

Figure 3.2: Schematic of the LCLS beamline through the first bunch compressor at the time of measurements (figure from Ref. [1]). The current beamline is nearly identical except for the addition of a laser heater in the dogleg following $\mathrm{LOb}$.

with the camera collection angle $\theta_{m}$, setting $R_{51}=R_{52}=0$ and using $R_{11}, R_{12}, R_{33}, R_{34}$ from the LCLS design lattice. Because the gain depends sensitively on the slice energy spread $\sigma_{\delta 0}$ (experimentally unknown), we match the theoretical gain curve to the experimental one using a slice rms energy spread of $3 \mathrm{keV}$ (Fig. 3.5). This $3 \mathrm{keV}$ value is consistent with the typical slice energy spread measured from a photocathode rf gun [54]. We suspect that the discrepancy at low energy arises from regions of smaller energy spread in which the short wavelength microbunching survives.

We also use Eqs. (3.53) and (3.54) at different QB settings to calculate the width of the QB curve at $\lambda=1 \mu \mathrm{m}$, near the long wavelength end of the OTR camera's bandwidth. The absence of the transverse beam size $\left(\sigma_{x}\right)$ in the final 3D result lowers QB sensitivity, but we still predict a width about a factor of 2 narrower than in the measured QB curve (Fig. 3.3). We suspect the larger width may be related to the non-smooth transverse electron distributions generated by laser and/or cathode non-uniformity.

\subsubsection{Comparison of 6D and 2D Models}

We would like to compare our $6 \mathrm{D}$ result to the equivalent prediction of a 2D model of shot noise. To do this, we return to the short impedance approximation (Eq. 3.53). First, we quote the result of a self-consistent 2D shot noise model; as in the $6 \mathrm{D}$ version, we use the same electron distribution for bunching and modulation, 


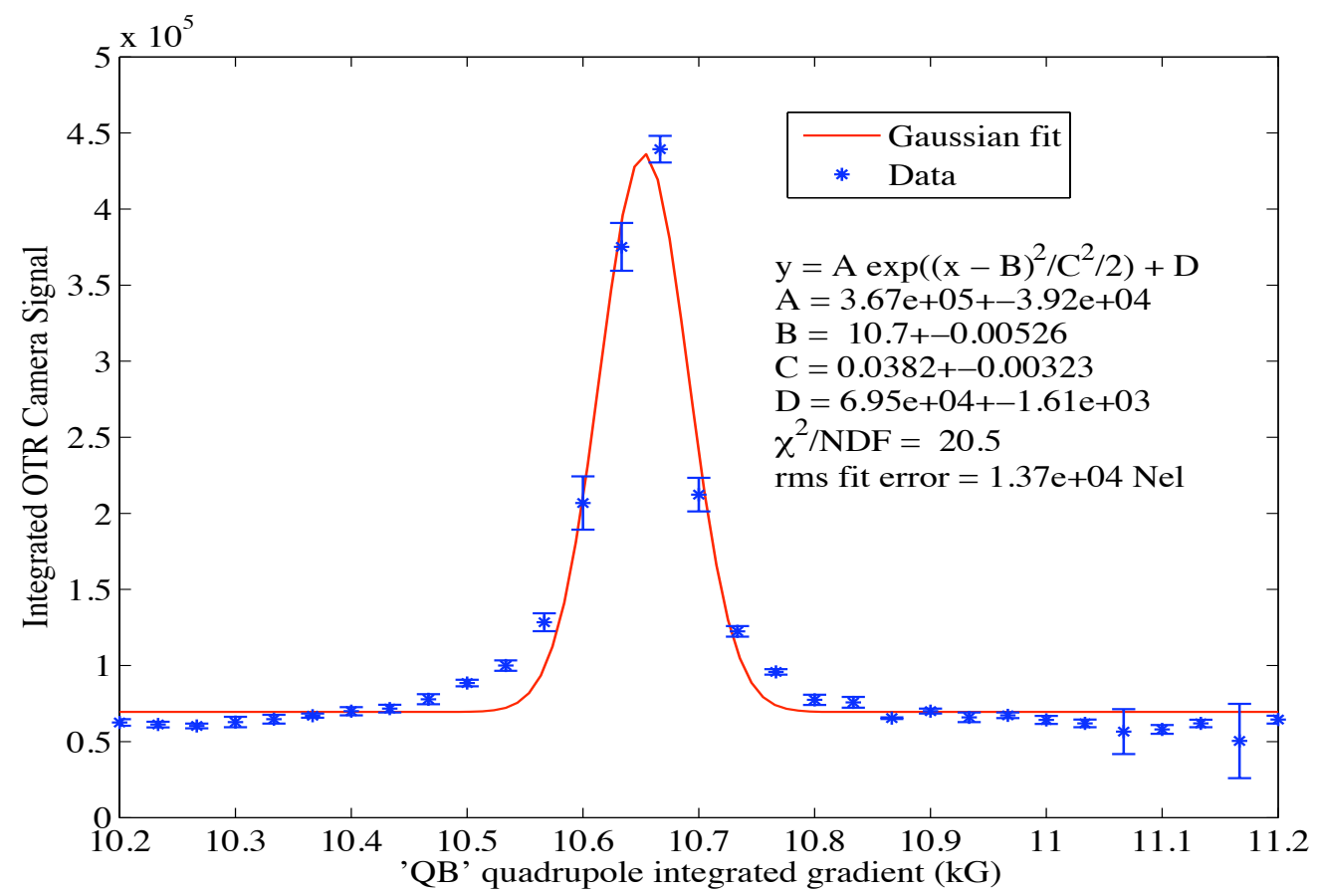

Figure 3.3: The integrated OTR signal as a function of the QB quadrupole strength at $250 \mathrm{pC}$ bunch charge (courtesy D. Dowell et al.).

but now with only longitudinal shot noise, and smooth transverse distribution, $S(Y)$,

$$
\rho(\boldsymbol{X})_{2 \mathrm{D}}=\frac{S(Y)}{N S_{n}} \sum_{z_{j}}^{N} \delta\left(z^{\prime}-z_{j}\right),
$$

with normalization $S_{n}=\int d x d y S(Y)$. Following the same calculation as for the $6 \mathrm{D}$ model we find

$$
\begin{aligned}
\left\langle\left|\bar{E}_{z}(s)\right|^{2}\right\rangle_{2 \mathrm{D}}= & \left(\frac{e k n_{0}}{4 \pi \epsilon_{0} \gamma^{2}}\right)^{2}\left(\frac{\gamma^{2}}{k^{2} \sigma^{2}}\right)^{2} \exp \left[-k^{2} R_{56}^{2} \sigma_{p}^{2}\right] \exp \left[-\frac{k^{2} \sigma^{2}\left(R_{1}^{2}+R_{33}^{2} \theta_{y}^{2}\right)}{2}\right] \\
& \exp \left[-k^{2} \sigma^{\prime 2}\left(R_{2}^{2}+R_{34}^{2} \theta_{y}^{2}\right)\right]\left[\int r d r K_{0}\left(\frac{k r}{\gamma}\right) J_{0}\left(-\frac{A k r}{2}\right) \exp \left[-\frac{r^{2}}{4\left\langle x^{2}\right\rangle}\right]\right]^{2}
\end{aligned}
$$

and bunching in the high frequency limit

$$
\left\langle|b(\boldsymbol{k})|^{2}\right\rangle_{2 \mathrm{D}}=\left[\frac{I}{I_{A} \gamma} \frac{R_{56} L}{\sigma^{2}}\right]^{2} \frac{e^{-k^{2}\left[R_{56}^{2} \sigma_{p}^{2}+\frac{1}{2} \sigma^{2}\left(R_{1}^{2}+R_{33}^{2} \theta_{y}^{2}\right)+\sigma^{\prime 2}\left(R_{2}^{2}+R_{34}^{2} \theta_{y}^{2}\right)\right]}}{\left[\frac{\gamma^{2} R_{q}^{2}}{4}+1\right]^{2}} .
$$

The sensitivity to off-diagonal elements in the $\mathrm{R}$ matrix is different, but the form is generally the same. We may also imagine an even simpler 2D model, using a transverse Gaussian for the electrons, but a uniform transverse E-field for modulation. The simpler E-field simplifies the algebra, giving

$$
\left\langle|b(\boldsymbol{k})|^{2}\right\rangle_{C}=\left[\frac{I}{I_{A} \gamma} \frac{R_{56} L}{\sigma^{2}}\right]^{2} e^{-k^{2}\left[R_{56}^{2} \sigma_{p}^{2}+\sigma^{2}\left(R_{1}^{2}+R_{33}^{2} \theta_{y}^{2}\right)+\sigma^{\prime 2}\left(R_{2}^{2}+R_{34}^{2} \theta_{y}^{2}\right)\right]} .
$$

To compare the gain, we take the achromatic limit (only $R_{56} \neq 0$ for both 6D (Eq.3.51) and 2D (Eq.3.56) models (Fig.3.6). (Both 2D models predict the same gain curve.) For low frequency bunching, the 6D and 


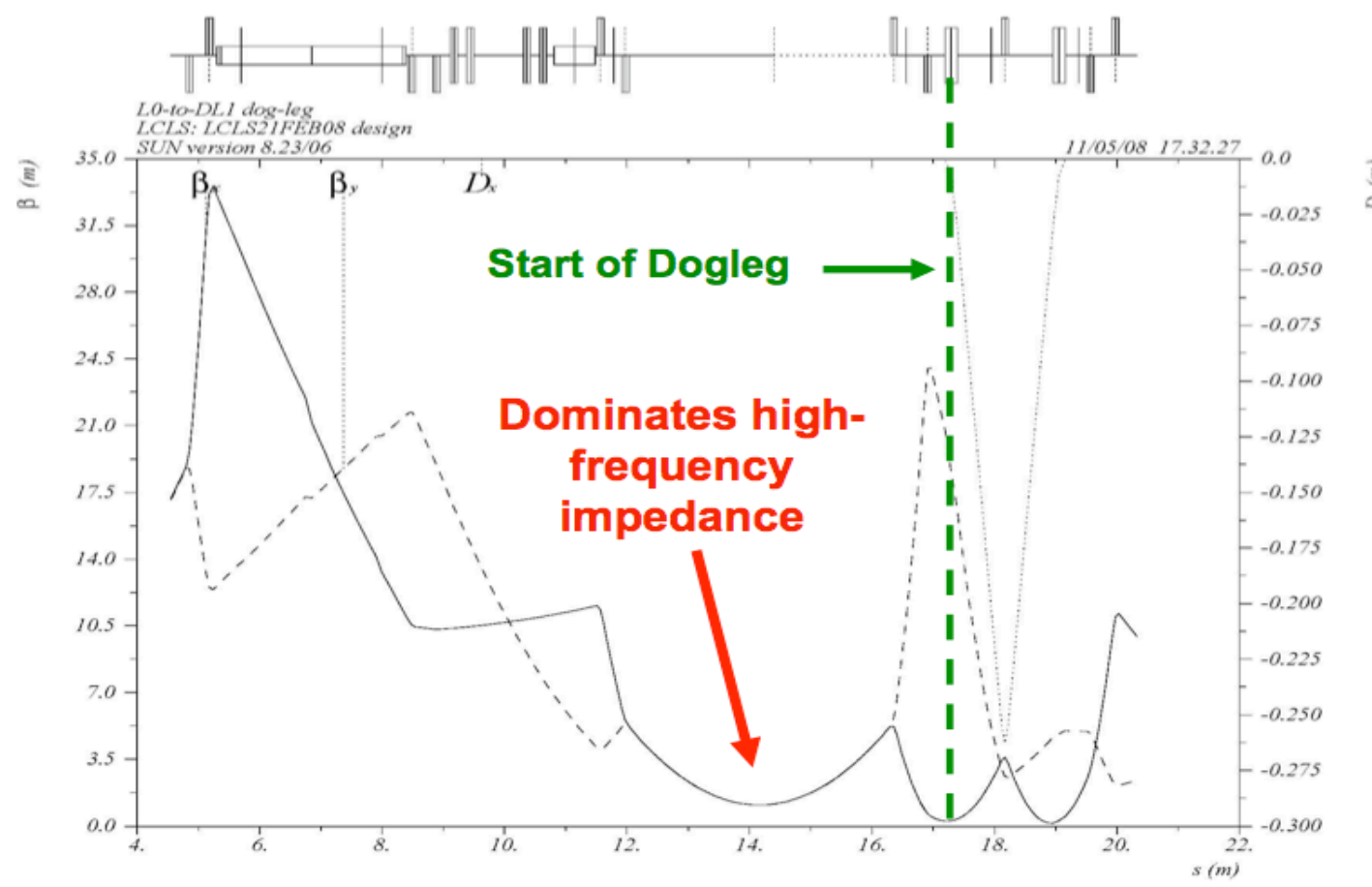

Figure 3.4: Twiss parameters for the LCLS beamline showing the small waist in front of the dogleg. Experimental attempts to decrease or increase the waist were not successful, likely because second order effects resulting from changes to the large beta function in the dogleg dominate.

2D models converge, but in the high frequency limit the 6D model predicts stronger bunching by a factor of $4 / 3$.

We can also guess that the models predict different sensitivity to chromatic affects. In particular, our 6D model has no exponential dependence on $R_{q} \equiv \sqrt{R_{1}^{2}+\theta_{y}^{2} R_{33}^{2}}$. When $\beta$ is large, and thus $\sigma^{\prime}=\sigma / \beta$ is small, we can drop the $R_{2}$ and $R_{34}$ dependence. In this regime, we expect the Lorentzian dependence of the $6 \mathrm{D}$ model to differ significantly from the $2 \mathrm{D}$ prediction. To experimentally validate the $6 \mathrm{D}$ results, we require a beam with a short impedance section (to ignore s-dependence and transverse motion), large $\beta$ (to suppress angular effects), and low $\gamma$ (to ensure 6D regime). However, we cannot have such low $\gamma$ that velocity bunching dominates.

\subsubsection{Comparison of Linear and Quadratic Terms}

We would like to compare the linear term to the quadratic term in the case of a short impedance section (for simplicity). Taking the case of a transversely frozen beam (short impedance section), we drop the $s$ integral and take the same transport matrix as for the quadratic term. The angular component is just a Gaussian 


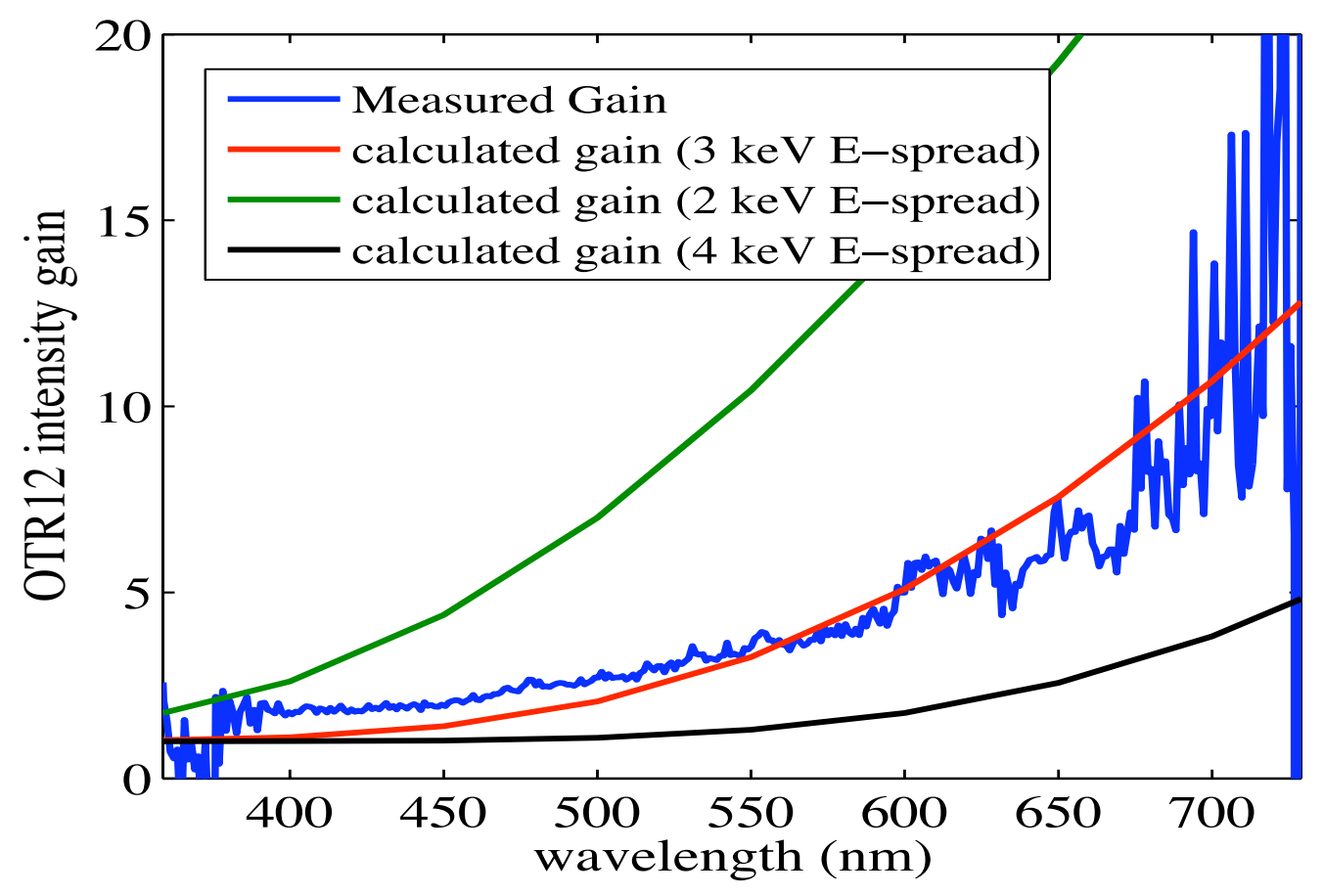

Figure 3.5: OTR intensity gain for $250 \mathrm{pC}$ charge at OTR12 as a function of the optical wavelength.

integral, but with $\sigma \rightarrow \sqrt{2} \sigma$. Integrating over both angular components $\left(x^{\prime}, y^{\prime}\right)$ gives

$$
\begin{aligned}
\left\langle|b(\boldsymbol{k})|^{2}\right\rangle_{(\delta)} & =n_{0} R_{56} k^{2} \frac{2 I}{I_{A}} e^{-k^{2} R_{56}^{2} \sigma_{p}^{2}} \frac{e^{-k^{2} \sigma^{\prime 2}\left(R_{2}^{2}+\theta_{y}^{2} R_{34}^{2}\right)}}{4 \pi \sigma^{2}} \int r d r d \phi e^{\left[-\frac{r^{2}}{4 \sigma^{2}}\right]} e^{i \tilde{K} R_{q} r(\cos \phi, \sin \phi)} K_{0}\left(\frac{k r}{\gamma}\right) \\
& =n_{0} R_{56} k^{2} \frac{2 I}{I_{A}} e^{-k^{2} R_{56}^{2} \sigma_{p}^{2}} \frac{e^{-k^{2} \sigma^{\prime 2}\left(R_{2}^{2}+\theta_{y}^{2} R_{34}^{2}\right)}}{2 \sigma^{2}} \int r d r J_{0}\left(k r R_{q}\right) e^{\left[-\frac{r^{2}}{4 \sigma^{2}}\right]} K_{0}\left(\frac{k r}{\gamma}\right) \\
& =n_{0} R_{56} k^{2} \frac{2 I}{I_{A}} e^{-k^{2} R_{56}^{2} \sigma_{p}^{2}} \frac{e^{-k^{2} \sigma^{\prime 2}\left(R_{2}^{2}+\theta_{y}^{2} R_{34}^{2}\right)} \gamma^{2}}{2 \sigma^{2} k^{2}} \frac{1}{\gamma^{2} R_{q}^{2}+1},
\end{aligned}
$$

with the usual limit $k \sigma / \gamma \gg 1$ and definitions $R_{q} \equiv \sqrt{R_{1}^{2}+\theta_{y}^{2} R_{33}^{2}}, R_{1} \equiv R_{51}+\theta_{x} R_{11}, R_{2} \equiv R_{52}+\theta_{x} R_{12}$. We then find

$$
\left\langle\sum_{l \neq j}^{N} \sum_{j}^{N} e^{-i \tilde{K} R\left(X_{j}-X_{l}\right)} i k R_{56} \delta_{j}\right\rangle=A \frac{e^{-k^{2}\left[R_{56}^{2} \sigma_{p}^{2}-\left(R_{2}^{2}+\theta_{y}^{2} R_{34}^{2}\right) \sigma^{\prime 2}\right]}}{\gamma^{2} R_{q}^{2}+1},
$$

with

$$
A \equiv \frac{R_{56} L}{\sigma^{2}} \frac{I}{I_{A} \gamma}
$$

The complementary $\delta_{l}$ term is exactly equivalent, but with a sign change in the Fourier transform. ( $k \rightarrow-k$, because the phase is now $e^{i k z_{l}}$ instead of $e^{-i k z_{j}}$.) With the sign flip, the $\delta_{j}$ and $\delta_{l}$ terms add in phase, and we find

$$
\left\langle\sum_{l \neq j}^{N} \sum_{j}^{N} e^{-i \tilde{K} R\left(X_{j}-X_{l}\right)} i k R_{56}\left(\delta_{j}-\delta_{l}\right)\right\rangle_{6 \mathrm{D}}=2 A \frac{e^{-k^{2}\left[R_{56}^{2} \sigma_{p}^{2}-\left(R_{2}^{2}+\theta_{y}^{2} R_{34}^{2}\right) \sigma^{\prime 2}\right]}}{\gamma^{2} R_{q}^{2}+1}
$$




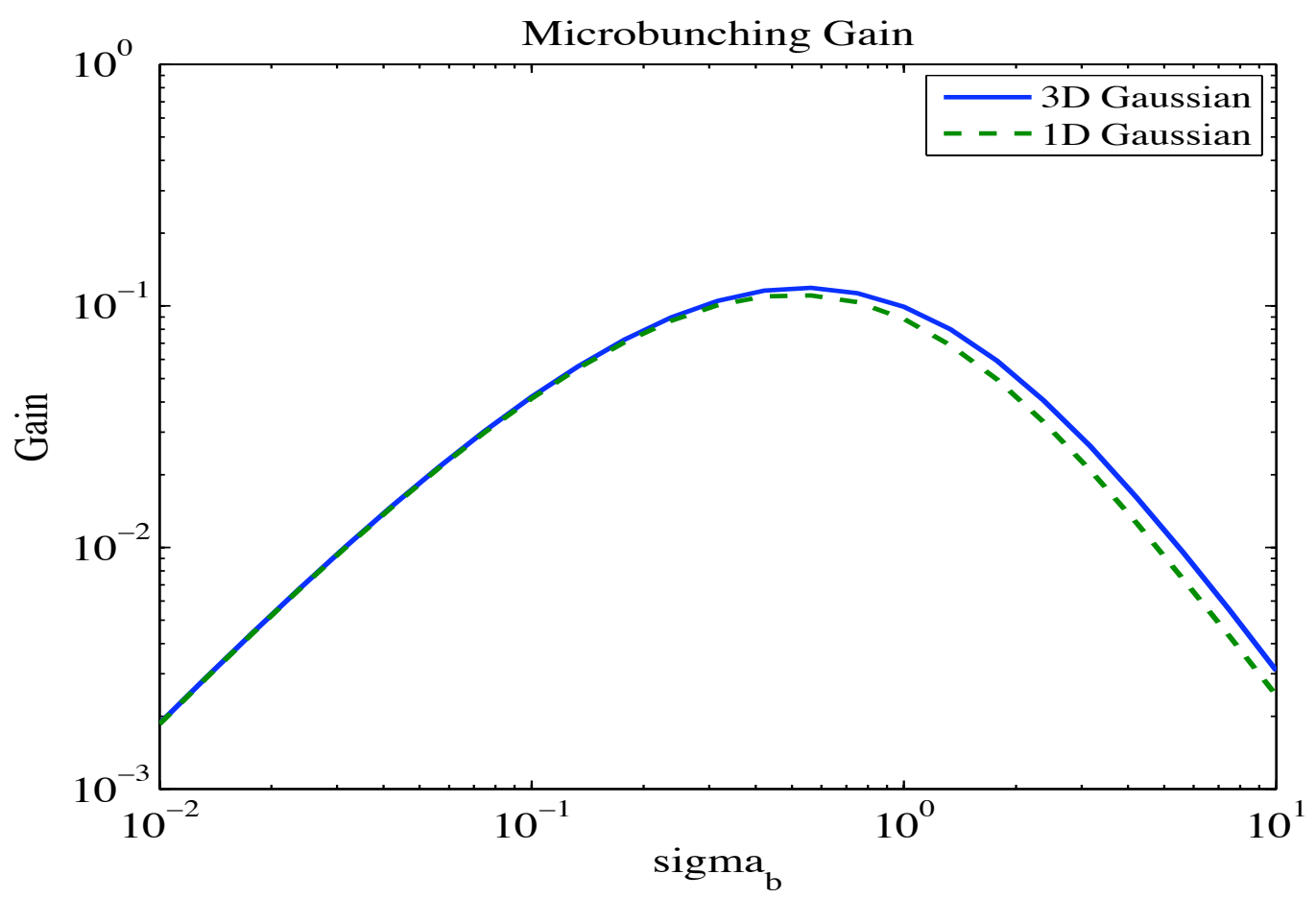

Figure 3.6: Total gain for 6D and 2D models (or 3D and 1D if we do not count angular components) is roughly equivalent. In the high frequency limit, the gains differ by a factor of $4 / 3$, due to our choice of gaussian transverse distribution. For flat-top distributions, the difference is even smaller (Fig. 2.15).

By comparison, for the quadratic $\delta_{j} \delta_{l}$ term we found previously

$$
\left\langle\sum_{l \neq j}^{N} \sum_{j}^{N} e^{-i \tilde{K} R\left(X_{j}-X_{l}\right)} i k R_{56} \delta_{j} \delta_{l}\right\rangle_{6 \mathrm{D}} \approx \frac{4 A^{2}}{3} \frac{e^{-k^{2}\left[R_{56}^{2} \sigma_{p}^{2}-\left(R_{2}^{2}+\theta_{y}^{2} R_{34}^{2}\right) \sigma^{\prime 2}\right]}}{\left[\gamma^{2} R_{q}^{2}+1\right]^{2}} .
$$

For the linear term to be comparable to the quadratic term in the limit of small suppression, we require $|A| \approx 1$. In general, this will be the case for large, low current beams at high energies. As a practical example, we take the microbunching observed after the first dogleg at LCLS [46]. In this case, $A \approx 25$, the Lorentzian suppression term is approximately 1 , and we can safely ignore the linear term.

When $A<0$ (e.g. for a chicane), the linear term can actually suppress bunching below the shot noise level. We then require not just $A \approx-1$, but also $e^{-k^{2} R_{56}^{2} \sigma_{p}^{2}} \approx 1$. For a $135 \mathrm{MeV}$ beam with $2.5 \mathrm{keV}$ energy spread $\left(\sigma_{p} \approx 2 * 10^{-5}\right)$ and $R_{56} \approx 5 \mathrm{~mm}$, we can have $n_{0} \approx 600 \mathrm{~nm}^{-1}$. This sets a lower limit on the wavelength range for which we could suppress microbunching. We note that with the LCLS parameters, reducing the $R_{56}$ by an order of magnitude would bring $|A| \approx 1$ while simultaneously diminishing the suppression term. We explore this concept more carefully in Chapter 2.

\subsection{Transverse Motion Examples}

We may apply Eqs. 3.39, 3.40 to predict the microbunching in a nearly arbitrary stretch of accelerator. The only limitation is that we assume dispersion is present in only one stretch of the accelerator, and that the 


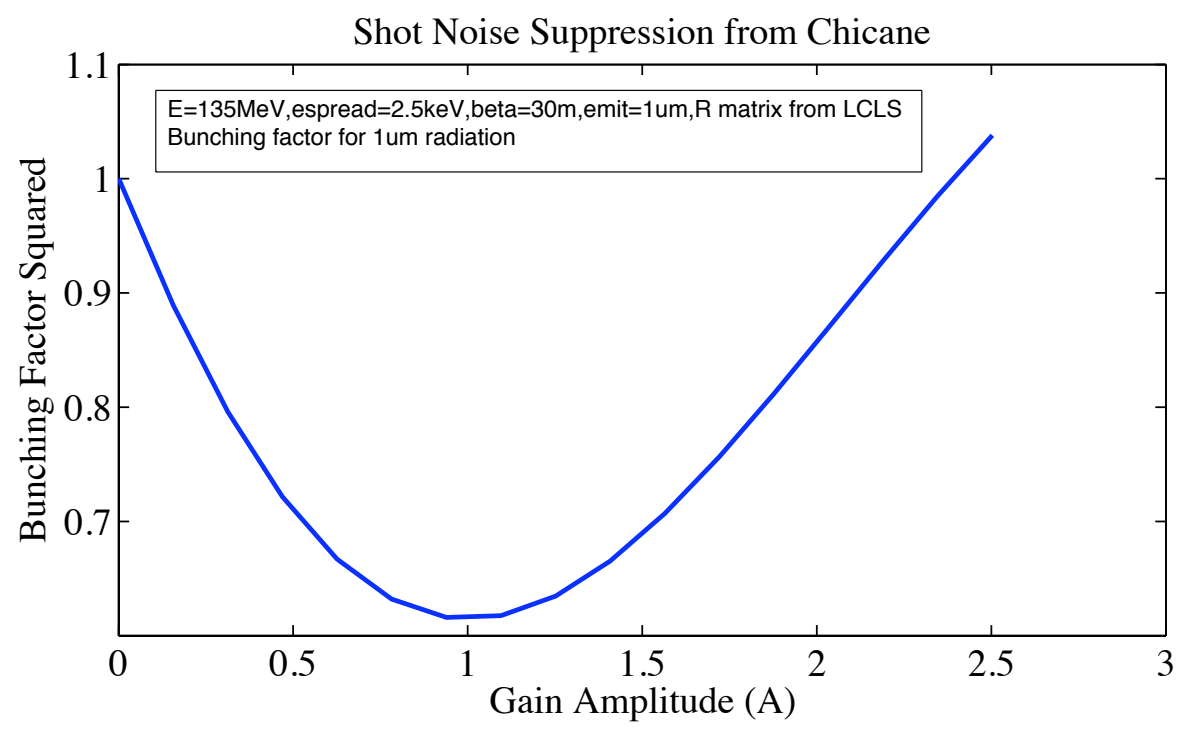

Figure 3.7: Suppression of shot noise from linear term. By setting $R_{56}$ so that the linear term has a negative contribution to the noise, we can suppress the bunching factor below the shot noise level.

impedance is negligible in that region. In principle, we can then apply our model to realistic accelerator lattices and include transverse effects due to finite emittance. (We consider only first order transport here, though it is possible to account for some second order effects as well.)

As a simple example including transverse motion, we first consider a drift space. Normalizing to the beam waist for the accelerator coordinate, $\tilde{s}=s / \beta$, and electron angle coordinates, $\tilde{x}^{\prime}, \tilde{y}^{\prime}=\beta x^{\prime}, \beta y^{\prime}$, we have transfer function

$$
R(0 \rightarrow \tilde{s})=\left(\begin{array}{cccc}
1 & s & 0 & 0 \\
0 & 1 & 0 & 0 \\
0 & 0 & 1 & s \\
0 & 0 & 0 & 1
\end{array}\right)
$$

As in the short impedance section, we have Jacobian $\mathbf{J}=1$. We can now plug $R$ into Eq. 3.40, and solve for the bunching factor. This analysis is partially complete, but due to the algebraic complexity we do not present it here. We note that the equations, though involving many terms, are straightforward to integrate in Mathematica.

At the beginning of the accelerator we are in the regime of strong acceleration. With $\gamma=\gamma_{0}(1+\alpha s)$, we have $\alpha s \gg 1$, but $s \ll 1 / k_{\beta}$, and we find

$$
x(s) \approx x_{f}-\frac{\gamma_{f}}{\gamma_{0} \alpha} \ln \left(\frac{\gamma(s)}{\gamma_{f}}\right) x_{f}^{\prime}
$$

where $\gamma_{0}$ is the initial energy, $\gamma_{f}$ is the final energy, and $\alpha$ is the accelerating gradient. If we define $G(s) \equiv$ $\frac{\gamma_{f}}{\gamma_{0} \alpha} \ln \left(\frac{\gamma_{f}}{\gamma(s)}\right)$, then we have

$$
x(s)=x_{f}+x_{f}^{\prime} G(s),
$$

which is the same form as for a drifting beam, but with the transformation $s \rightarrow G(s)$. We then expect that, until we carry out the $s$ integrals, the two results should be identical beyond this substitution. 
Finally, we take simple betatron motion (with acceleration). Again normalizing the accelerator coordinate, $\tilde{s}=s / \beta$, and electron angle coordinates, $\tilde{x}^{\prime}, \tilde{y}^{\prime}=\beta x^{\prime}, \beta y^{\prime}$, we have transfer function

$$
R(0 \rightarrow \tilde{s})=\sqrt{\frac{\gamma_{0}}{\gamma_{s}}}\left(\begin{array}{cccc}
\cos (\tilde{s}) & \sin (\tilde{s}) & 0 & 0 \\
-\sin (\tilde{s}) & \cos (\tilde{s}) & 0 & 0 \\
0 & 0 & \cos (\tilde{s}) & \sin (\tilde{s}) \\
0 & 0 & -\sin (\tilde{s}) & \cos (\tilde{s})
\end{array}\right)
$$

giving Jacobian, $\mathbf{J}(\tilde{s})=\left(\gamma_{0} / \gamma_{s}\right)^{2}$. Again, we may plug into Eq. 3.40 and integrate directly in Mathematica.

These examples have not been pushed to completion due to the algebraic complexity and absence of appropriate experimental comparison. However, in principle all cases may be evaluated with a combination of analytical and numerical integrals.

\subsection{Discussion}

Though the transverse and longitudinal models converge in the low frequency limit, our 6D shot-noise model predicts different properties in the high frequency regime. When the transverse coherence length is smaller than the beam size $(\lambda / \gamma \ll \sigma)$, we expect the bunching properties to be determined by the coherence length, rather than the beam size, as predicted by the classic 2D model. In particular, the microbunching should be less sensitive to spatial chromatic effects $\left(R_{51}, R_{53}\right.$, etc.). Second, our model has the inherent benefit of self-consistency; the same electron distribution is used for modulation and bunching. Finally, we note that in principle the model can be used to calculate MBI effects for arbitrary beam elements (assuming no dispersive regions), including emittance effects. 


\section{Chapter 4}

\section{FEL Gain Length and Taper Measurements at LCLS}

\subsection{Introduction}

In this chapter we present gain length measurements from LCLS, the world's first hard X-ray laser in operation since April, 2009 [55]. LCLS recorded first lasing on April 10th, 2009, with the first gain lengths and FEL saturation measured four days later. In this paper we describe the methods used to measure gain lengths and post-saturation power growth. We also present results of the first gain length measurements for LCLS. The results of this chapter were first presented in Ref. [56].

\subsection{Gain Length Measurements}

In the linear regime, after the start-up from shot noise and before saturation, FEL theory predicts an exponential growth in power, $P(z) \propto e^{z / L_{G}}$, with gain length $L_{G}$. In simple 1-D theory, the gain length can be estimated as

$$
L_{G}=\lambda_{u} / 4 \pi \sqrt{3 \rho}
$$

with Pierce parameter

$$
\rho \equiv\left[\frac{I}{8 \pi I_{A}}\left(\frac{K[J J]}{1+K^{2} / 2}\right)^{2} \frac{\gamma \lambda_{r}^{2}}{2 \pi \sigma_{x}^{2}}\right]^{1 / 3}
$$

where we have used the undulator strength parameter, $K=0.94 B_{0}[$ Tesla $] \lambda_{u}[\mathrm{~cm}]$, scaling parameter for planar undulators, $[\mathrm{JJ}]=J_{0}\left(K^{2} /\left(4+2 K^{2}\right)\right)-J_{1}\left(K^{2} /\left(4+2 K^{2}\right)\right)$ defined in terms of Bessel functions, $J_{0}$, peak current, $I$, Alfvén current, $I_{A}$, electron radius, $\sigma_{x}$, relativistic factor, $\gamma$, and resonant wavelength, $\lambda_{r}$ [11]. LCLS has a conservative beam design, with enough undulators to operate deep in the saturation region. Due to the slow power growth in saturation, the final FEL power may not depend sensitively on the electron beam quality. Instead, we use the gain length as a gauge of total FEL performance. FEL experiments may 
also work optimally near to saturation, so it is useful to know the power profile for both machine development and user experiments. All measurements described in this chapter use the gain length GUI (Section 4.5).

\subsubsection{Undulator Removal Method}

To determine the gain length, we characterize the FEL pulse energy as a function of position along the undulator line. Due to practical considerations, all diagnostics follow the last undulator, so we cannot measure the pulse energy within the undulator line. Consequently, to determine power as a function of position, we must disrupt the FEL process as the electrons travel along the undulators. With $3.35 \mathrm{~m}$ long undulator segments and soft X-ray gain lengths as short as $1.5 \mathrm{~m}$, we would ideally measure the pulse energy following each undulator segment.

The most direct method for suppressing the FEL is to remove undulators sequentially, allowing the electrons to drift through the remaining length of the undulator hall. Removing undulators has the added benefit of decreasing the spontaneous background signal, which increases linearly with undulator length. The drop in spontaneous background benefits measurements near the beginning of the undulator line, where FEL power level is many orders of magnitude weaker than at saturation. However, each undulator requires 3 minutes to remove (more than 90 minutes for a full $P(z)$ scan), so initial concerns about the temporal FEL stability prompted interest in an alternative method. Additionally, the gain length measurement was envisioned as a performance gauge for the FEL, and even with current stable operation, 90 minutes is too long for a routine operator measurement.

\subsubsection{Transverse Kick Method}

Rather than removing undulators, we can instead disrupt the FEL process. For example, introducing a distortion to the electron orbit suppresses the FEL by decreasing bunching and beam overlap [57] (Fig. 4.1). The electrons still produce spontaneous radiation as they travel through the undulator line, but the poor orbit disrupts the delicate FEL process. By kicking the beam transversely at sequential positions in the undulator hall, we can then measure the FEL gain length.

Following each LCLS undulator segment, a pair of $x$ and $y$ dipole correctors can kick the beam by approximately $15 \mu \mathrm{rad}$ in each plane. The requirement for FEL suppression is determined by the critical angle, $\phi_{c}=\sqrt{\lambda_{r} / L_{G}}$, with FEL wavelength $\lambda_{r}$ and gain length, $L_{G}$. For hard X-rays at LCLS, $\phi_{c} \approx 7 \mu \mathrm{rad}$, so the dipole correctors can strongly suppress the FEL process in the downstream undulators. The kick method is less effective at longer wavelengths, when the beta function is smaller and the critical angle is larger. Using the kick method, operators can complete a full $P(z)$ scan in under 10 minutes. We show good agreement between the two methods at $1.5 \AA$ in Fig. 4.2.

\subsubsection{Restarting FEL with Kick Method}

Though the kick method is faster and generally equivalent in accuracy to the undulator removal method, we note two drawbacks. First, the background signal at low power (when the beam is kicked early in the FEL process) is larger than in the undulator removal method, where the spontaneous background is proportional to the number of inserted undulators. Second, distorting the orbit near the beginning of the undulator hall may allow the FEL process to restart, leading to secondary (though weak) FEL spots (Fig. 4.3). We observe 


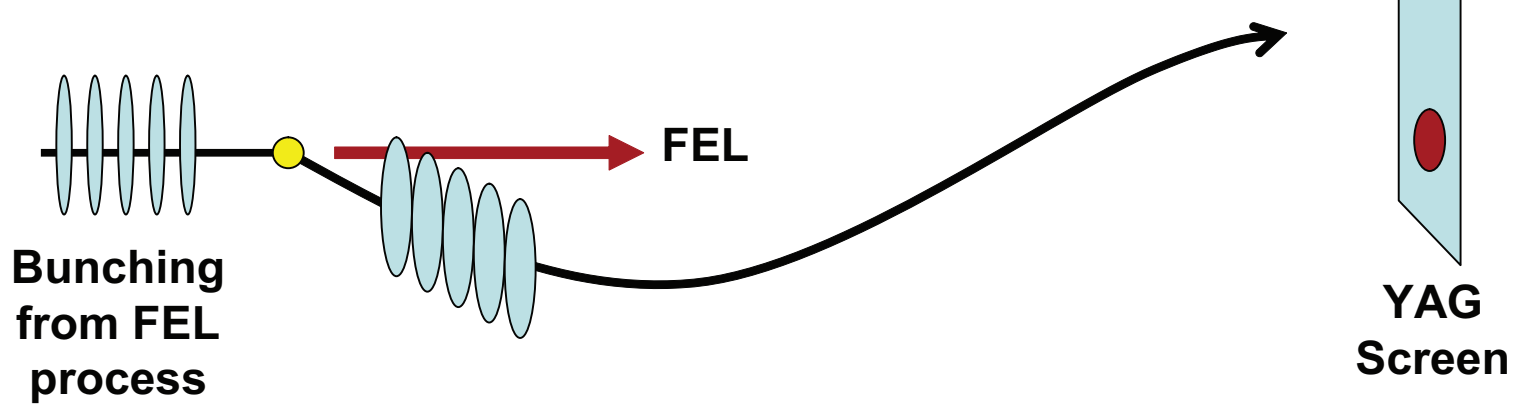

Figure 4.1: A transverse dipole kick suppresses the FEL process. The electrons continue to travel through the undulator line, but the kick smears the bunching and misaligns the radiation and electron motion.

restart occurring in two places: after a $\pi / 2$ phase advance and further downstream when the orbit distortion straightens (Fig. 4.4). An additional kick further down the undulator (for instance at slightly more than a $\pi / 2$ phase advance) effectively suppresses both secondary spots. Given the spatial separation of the spots (as seen in the cartoon, Fig. 4.4), an alternative solution is to select a sufficiently small region of interest in the camera, so that we only record the primary FEL spot.

\subsubsection{X-ray Power Data Collection}

To measure the FEL pulse energy, we insert a fluorescent YAG screen into the path of the FEL. The primary diagnostic for the initial gain length measurements is the YAGXRAY screen located $50 \mathrm{~m}$ downstream from the last undulator. We determine X-ray power by summing pixels within a $5 \sigma$ region of interest around the FEL spot. We also compare the simple summing analysis to various fitting methods (RMS, cut RMS, etc.) and observe little difference. Two neutral density filters with a total transmission of $0.1 \%$ extend the camera's dynamic range to more than 4 orders of magnitude.

A more complex suite of diagnostics exists in the Front End Enclosure (FEE). The direct imager is a set of YAG screens of thicknesses ranging from $5 \mu \mathrm{m}$ to $1 \mathrm{~mm}$. Two cameras, a narrow field of view (NFOV) and wide field of view (WFOV), can image any of the screens. For gain length measurements we typically use the $100 \mu \mathrm{m}$ thick YAG screen, though the $1 \mathrm{~mm}$ screen is needed for harmonic measurements of hard $\mathrm{X}$-rays. In addition to the YAG screen there are two gas detectors, a total energy monitor (currently inactive) and a group of solid and gas X-ray attenuators that prevent YAG saturation. The attenuators are particularly important for soft X-ray measurements, when the intense FEL pulse (absorbed in the a thin surface layer) has the potential to damage the YAG screen.

Though the direct imager was designed to be superior to the YAGXRAY imaging station, in practice it is difficult to extract a power measurement from the direct imager. The circular aperture created by the attenuators and gas detectors produces a disc of spontaneous radiation in the center of the direct imager screen. The FEL appears as a bright spot in the middle of the spontaneous radiation; as a result, a line-out shows the FEL spike raised above the pedestal of the spontaneous radiation. Due to contractual conflicts, the image analysis software was never finished, and with no zoom function on the camera hardware, the standard 


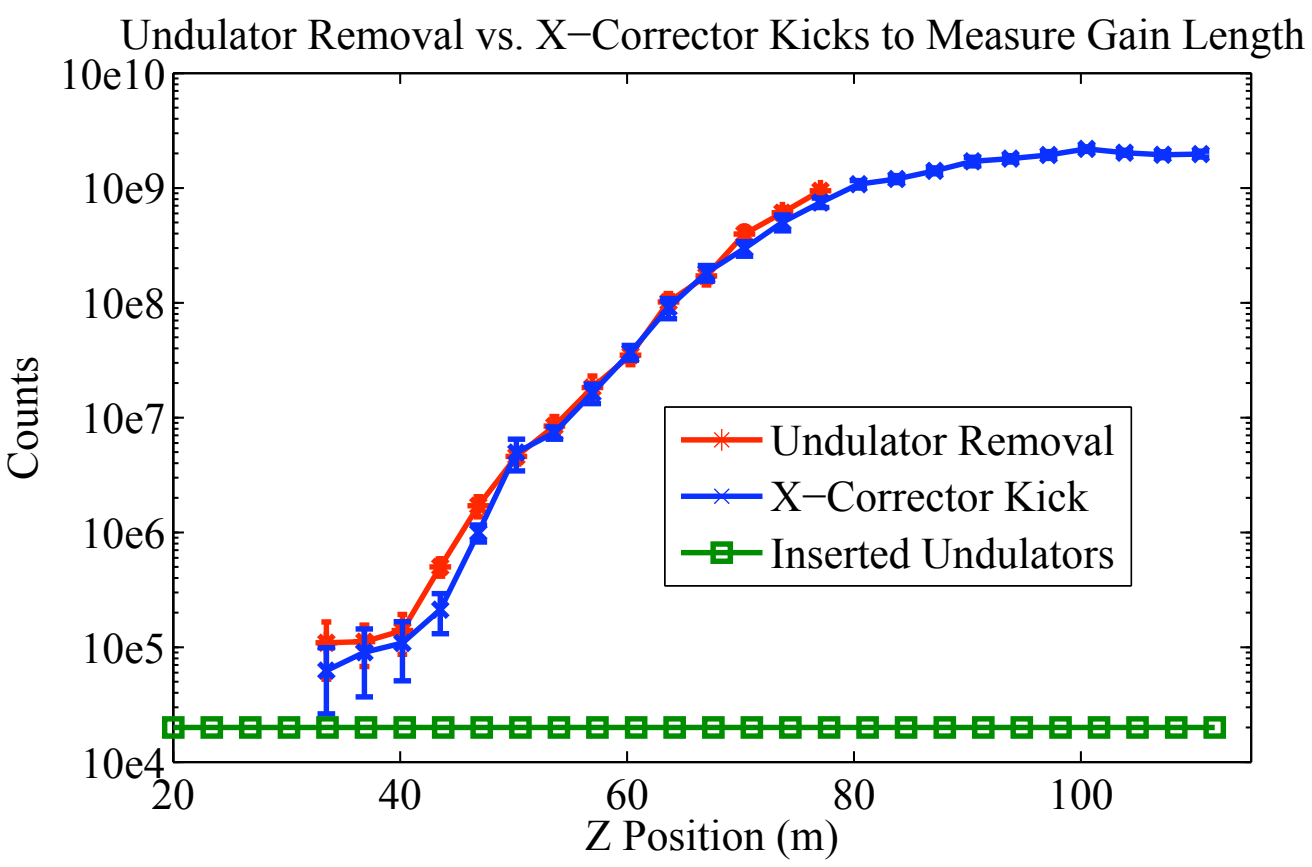

Figure 4.2: Gain lengths measured with both the undulator removal and dipole corrector kick methods. Distances are for total undulator length and do not include the breaks between segments.

peak finder must operate on the entire image (background, pedestal, and FEL spike). It is possible to subtract off the spontaneous pedestal by taking a background image with the FEL process suppressed (as described in section 4.2.2). However, the spontaneous subtraction is imperfect and slows the GUI. As a result, we prefer to use the YAGXRAY screen whenever possible.

\subsection{Results}

\subsubsection{LCLS Gain Length}

Due to the danger of damaging the YAGXRAY screen at low energies, we primarily study the gain length at $1.5 \AA$ radiation (13.6 GeV electron beam). After optimizing the electron beam parameters we measure gain lengths as short as $2.85 \pm 0.06 \mathrm{~m}$ at $250 \mathrm{pC}$ electron pulse charge (Fig. 4.5). More typically, we measure gain lengths between 3 and $4 \mathrm{~m}$, which agree with Genesis [58] simulation results for a beam with $0.4 \mu \mathrm{m}$ normalized emittance (Fig. 4.6). The shortest gain lengths may result from regions of very low emittance within the beam or from lasing in the wake-field induced current spikes (Fig. 4.7).

To measure gain lengths at $1.5 \mathrm{~nm}$ wavelength, we remove all but 9 undulators to reduce the overall power hitting the YAG screen. The FEL is harder to suppress at longer wavelength with the corrector kick; secondary FEL spots reflect off the beam pipe and overlap with the primary FEL. The undulator removal method is slow, but remains effective at all wavelengths. We measure a gain length of $1.62 \pm 0.15 \mathrm{~m}$ at 4.7 GeV beam energy (Fig. 4.8). 


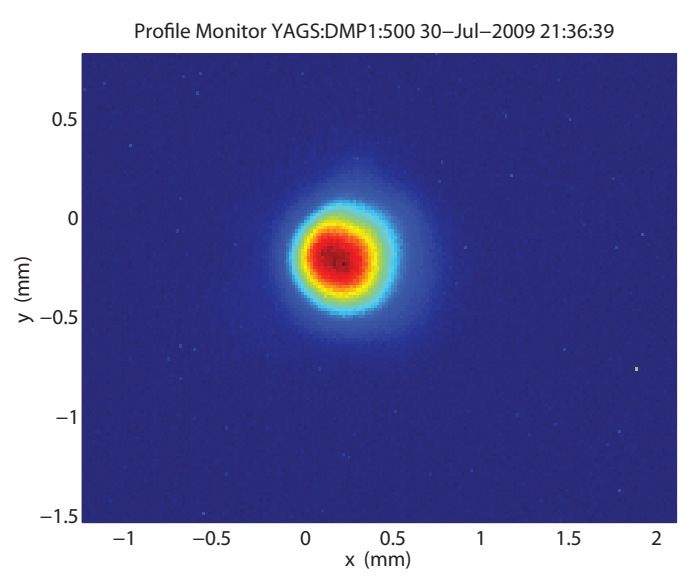

(a)

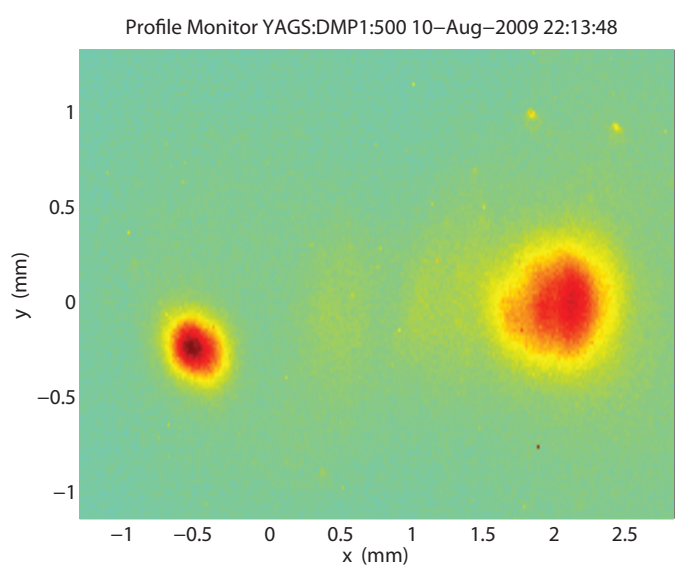

(b)

Figure 4.3: At left, the primary FEL spot at saturation is centered near $x=0.25 \mathrm{~mm}$. At right, after strongly suppressing the main FEL peak (faintly visible around $x \approx 0.5 \mathrm{~mm}$ ), secondary FEL spots emerge on either side. (Though stronger than the main FEL peak, these spots are still several orders of magnitude weaker than the saturated FEL). The left-hand secondary spot is aligned with the straight portion of the orbit distortion.

\subsubsection{Gain Length vs. Energy Spread}

With the kick method taking less than 10 minutes for a full $P(z)$ scan, we can measure gain length as a function of various electron beam parameters. In Fig. 4.9 we show one example: gain length vs. laser-heater induced energy spread. The laser heater produces an energy spread in keV of $\Delta E \approx 8 \sqrt{P_{L}}$, with the laser heater energy, $P_{L}$, in $\mu \mathrm{J}$ (Fig. 5.9) [4]. The final energy spread is then multiplied by the bunch compression factor, $\sim 90$ at $3 \mathrm{kA}$. The results are consistent with the Xie scaling [59] for 0.4-0.5 $\mu \mathrm{m}$ beam emittance. We also note that the gain length with the laser heater off $(0 \mathrm{keV})$ is approximately $1 \mathrm{~m}$ larger than at the nominal heater value $(20 \mathrm{keV})$. We attribute the increased gain length in the case of no laser heating to self-heating from the microbunching instability [22].

\subsubsection{Saturation Taper}

Tapering the undulator $\mathrm{K}$ parameter near and beyond saturation can increase the final FEL power [60, 61]. The resonant wavelength, $\lambda_{r}$, of an undulator is given by

$$
\lambda_{r}=\frac{\lambda_{u}}{2 \gamma^{2}}\left(1+K^{2} / 2\right)
$$

with undulator period, $\lambda_{u}$, undulator parameter $K$, and electron energy given by the relativistic factor $\gamma$. As the electrons lose energy to the radiation field, the resonance condition moves towards longer wavelengths. Changing the $K$ value compensates for energy loss and keeps the resonance condition fixed. (Each undulator has a $K$ range of approximately $1 \%$.) The energy loss may be due to incoherent effects (such as longitudinal wakefields and incoherent spontaneous emission) or to the substantial loss of energy from the saturated FEL process. We refer to tapering for the former as a 'gain' taper, and for the latter as a 'saturation' taper. The incoherent losses require use of a linear $K(z)$ gain taper across all undulators; we can calculate the optimal gain taper slope from electron beam parameters. To compensate for the additional FEL-induced energy loss, 


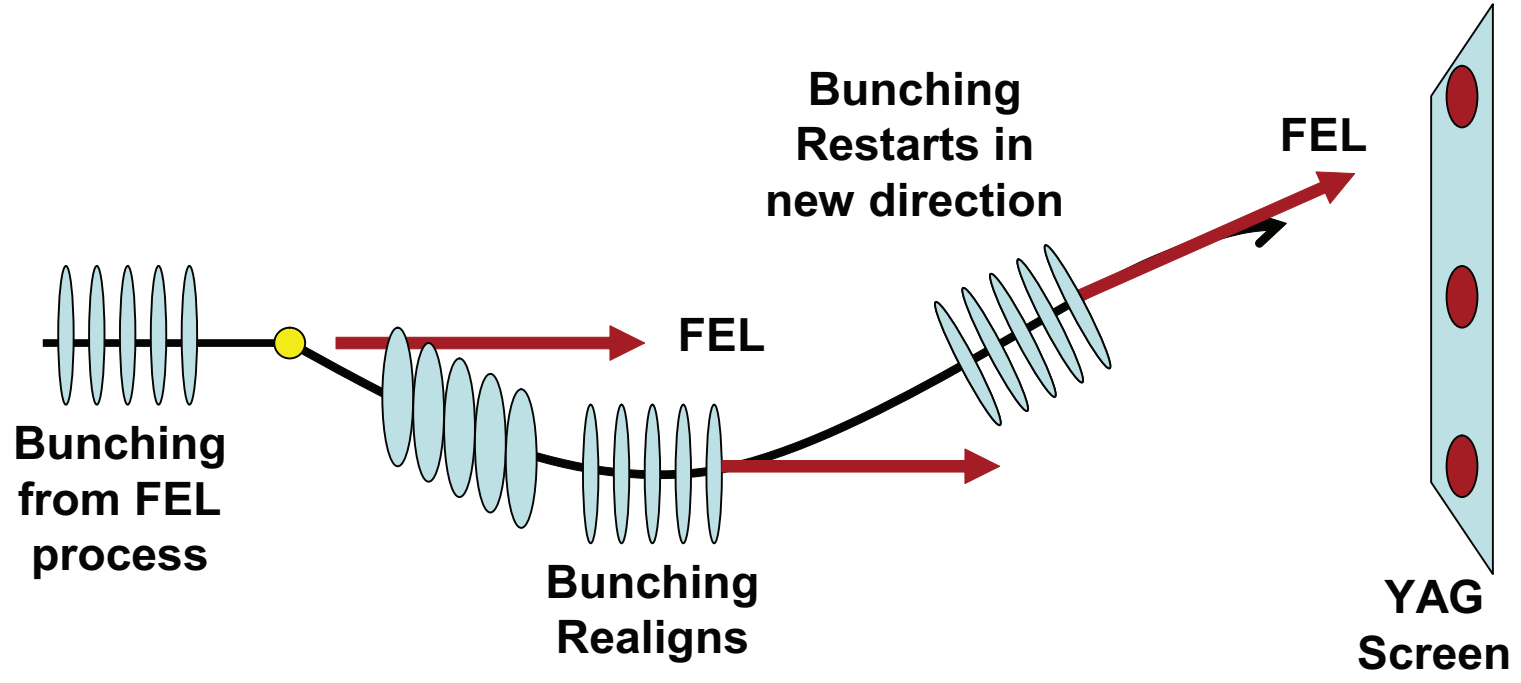

Figure 4.4: Cartoon showing the difficulty of suppressing the soft X-ray FEL. The kick temporarily stops the FEL process, but the bunching can either realign (lower spot) or can restart from shot noise during the straight portion of the distorted orbit (upper spot), creating secondary FEL radiation. Additional kicks can suppress this secondary radiation, or the camera region of interest can select only the primary central spot. In this cartoon, down corresponds to positive $\mathrm{X}$ on the YAG screen

we empirically scan linear 'saturation' tapers, changing both the slope and starting point, to find the optimal $K$ values (Fig. 4.10).

To evaluate our taper we use the same dipole corrector kick method as was used for the gain length studies. The YAGXRAY screen saturates before the FEL does, so we must infer the FEL power from the average electron energy loss, measured with beam position monitors (BPMs) in dispersive regions before and after the undulator line [5]. The kick method does not affect either spontaneous radiation or wakefields; consequently, any change in energy loss that correlates to a transverse kick must result from the FEL. The FEL-induced energy loss before saturation is small relative to the measurement noise, so this method is not effective for measuring the gain length.

Results from one such taper measurement, along with Genesis simulations [58] of the same LCLS parameters, are shown in Fig. 4.11. We compare the power gain relative to the un-tapered case for simulations and experiments (Fig. 4.12). Experimentally we observe a gain factor of 2.4, somewhat smaller than the factor of 3.3 found in Genesis simulations.

We can also compare the FEL saturation taper to the measured electron energy loss. The change in $K$ corresponds to a change in energy through the resonant condition, Eq. 4.3. The saturation taper of Fig. 4.10 matches a $35 \mathrm{MeV}$ change in electron energy, approximately four times the measured amplitude. The energy difference suggests that the saturation taper is increasing the FEL power emitted only by a small portion of the electron beam. We also observe that the total FEL power is relatively insensitive to the strength of the saturation taper; we may guess that as the taper strength increases as we withdraw more power from a small portion of the beam, leaving the total power unchanged. This picture is consistent with the results of Ref. [60].

If the tapering is only effective for a small portion of the beam, we expect that the radiation bandwidth will 


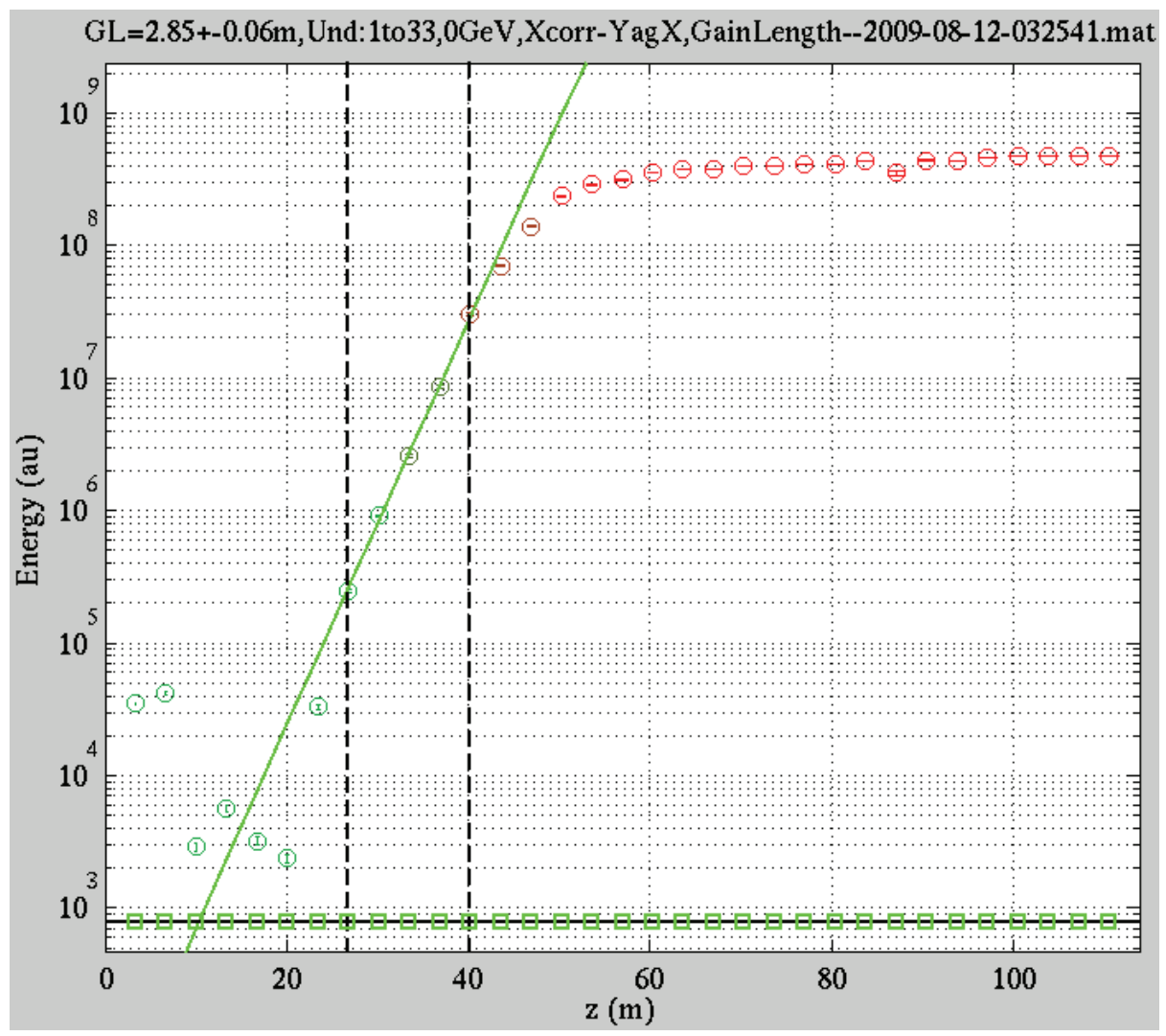

Figure 4.5: Sample gain length taken with the GUI showing gain length of $2.85 \pm 0.06 \mathrm{~m}$ at $13.6 \mathrm{GeV}$ using the dipole corrector method. The points within the dotted black lines are used to calculate the gain length (shown as solid green line). The high power levels of the first two data points are due to a secondary FEL spot as seen in Fig. 4.3. Colors of data points correspond to the state of the camera neutral density filters while taking the data. Green boxes represent inserted undulators.

increase as the wavelengths of the tapered and un-tapered portions diverge. If true, users requiring narrow bandwidths may choose to minimize the taper amplitude, or end the FEL process close to saturation. This subject requires further investigation.

\subsubsection{Undulator Gaps (self-seeding)}

The LCLS undulators are each $L_{u}=3.4 \mathrm{~m}$ long. Following each undulator, vacuum pumps, BPMs and beam finder wire diagnostics monitors occupy a gap of $L_{b} \approx 0.6 \mathrm{~m}$ before the next undulator. Because of the slight velocity difference between the electron and radiation, while traversing each gap the electrons fall behind the X-rays by $\Delta L=L_{b}(1 / \beta-1)$, with normalized electron velocity $\beta \equiv v_{e} / c$. We can ignore the gap slippage so long as the total difference, $\Delta L$, is approximately an integer multiple of the resonant wavelength, $\lambda_{r}=\frac{\lambda_{u}}{2 \gamma^{2}}\left(1+K^{2} / 2\right)$. Expressing the electron velocity in terms of the relativistic $\gamma$ factor, $1 / \beta \approx 1+1 / 2 \gamma^{2}$, we then find a condition on the gap length of

$$
L_{b}=n \lambda_{u}\left(1+K^{2} / 2\right)
$$




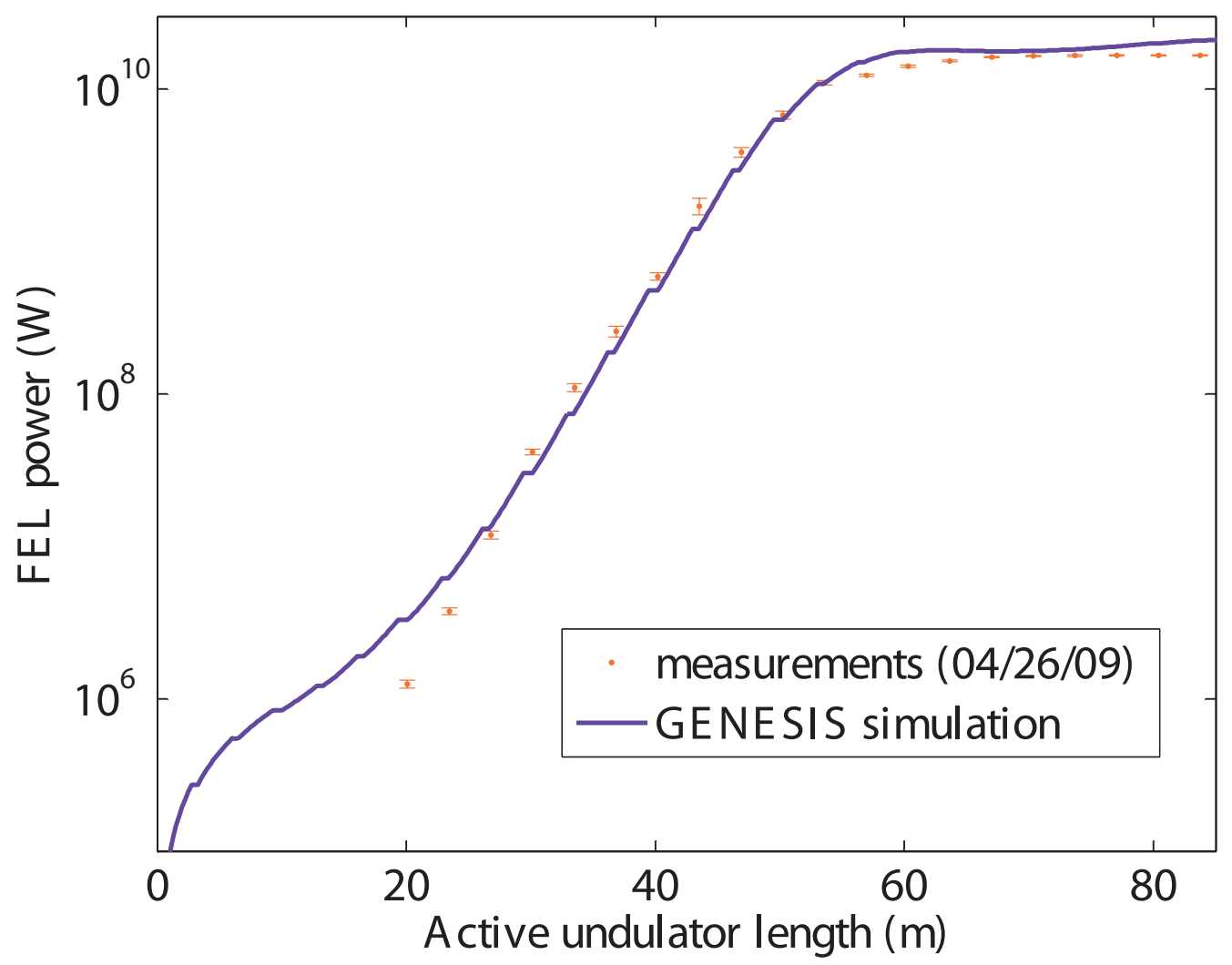

Figure 4.6: A gain length measurement of $3.3 \mathrm{~m}$ agrees well with Genesis simulations for a beam with 0.4 $\mu \mathrm{m}$ emittance.

for some integer $n$. Conveniently, the allowable gap lengths, $L_{b}$, do not depend on the electron energy, $\gamma$. (Indeed this is a motivation for tuning wavelengths by shifting $\gamma$ instead of shifting the undulator parameter $K$.) Consequently, LCLS does not need phase shifters (a delay to the electrons) to ensure that the electrons remain at the correct phase of the X-ray beam. With $L_{b} \approx 0.6 \mathrm{~m}$ (or $n=3$ ) the electron maintains its correct phase entering the next undulator section. Of course, the FEL power is constant in the drift section, so simulations show flat power growth between each undulator. (In fact, due to diffraction of the radiation during the gap, the power may even drop slightly. With only one measurement per undulator, it is not possible to experimentally confirm the behavior between undulators.)

For a test of a self-seeding scheme, LCLS plans to remove one undulator section and install a diamond crystal in its place [62]. During normal operation (crystal removed), the FEL then traverses one gap which is $4 \mathrm{~m}$ longer than the others. We expect the FEL to lose some power due to both diffraction in the gap as well as the mismatched phase following a gap which is no longer an integer multiple of $\lambda_{r}$. To test this effect experimentally, we used the gain length GUI to measure the power growth while removing sequential undulator sections. The results are given in Figs. 4.13.

As discussed above, changing the $K$ parameter (i.e. tapering) can correct for energy loss during FEL saturation. Alternatively, we can express the effect of the energy loss as a phase shift (see section 9). The resonant wavelength shifts as the energy decreases, resulting in larger slippage $\lambda_{s a t}=\frac{\lambda_{u}}{2 \gamma_{s a t}^{2}}\left(1+K^{2} / 2\right)>\lambda_{r}$. 


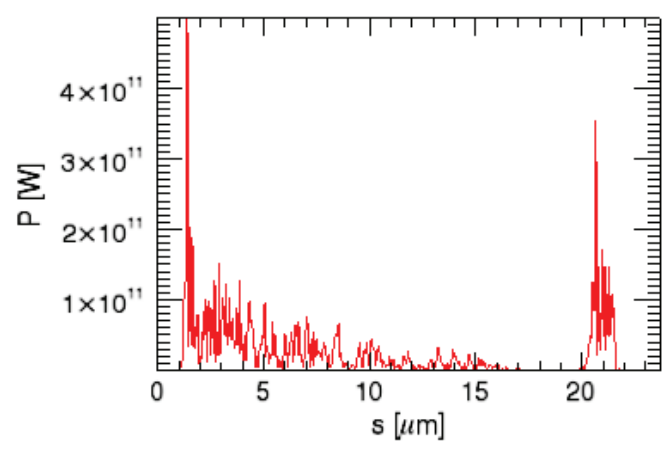

(a)

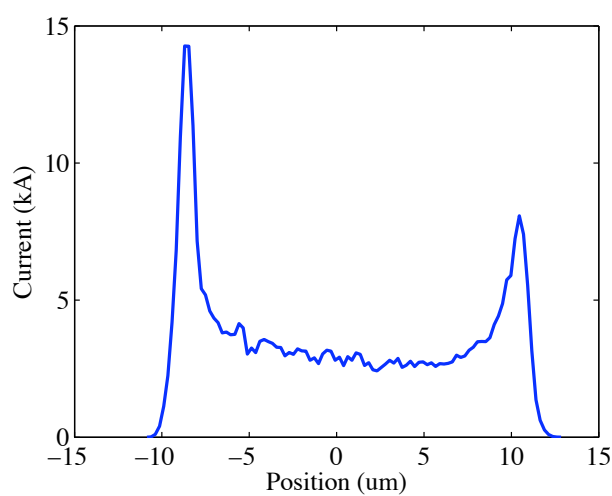

(b)

Figure 4.7: Genesis simulation showing power as a function of longitudinal bunch position (left). The peak power occurs in the current spikes (right). For this simulation, the spikes had emittances of $0.6 \mu \mathrm{m}$.

We can compensate the energy loss by either decreasing $K$ or by inserting phase shifts after each undulator section to maintain the phase relation between electrons and the original resonant $\mathrm{X}$-ray wavelength.

Conversely, a region with constant energy, but with a shift in $K$, will result in a shift in electron phase. From the resonant wavelength condition (Eq. 4.3), we find a phase shift rate of

$$
\frac{d \theta}{d z}=k_{u}-\frac{k_{r}}{2 \gamma^{2}}\left(1+K(z)^{2} / 2\right)
$$

with $k_{u} \equiv 2 \pi / \lambda_{u}$ and $k_{r} \equiv 2 \pi / \lambda_{r}$. For a shift in undulator parameter $\Delta K \equiv K-K_{0}$ over one undulator length, we find

$$
\Delta \theta=-2 k_{u} \frac{K_{0} \Delta K}{2+K_{0}^{2}} L_{u} \approx-2 \pi \frac{2 \Delta K}{K_{0}} N_{u}
$$

with $N_{u}=100$ periods per undulator. By shifting the undulators before and after the gap, we can induce an intentional phase shift, $\Delta \theta$, to compensate for the phase error from the missing undulator.

To test the effect of taper compensation, we scan the $K$ value of the undulators before and after the missing gap. We find that when the next undulator is inserted, shifting the $K$ value has little effect, but when the neighboring undulator is missing, shifting the $K$ value enhances the FEL power considerably (Fig. 4.14). The peak shift corresponds to shifting the electrons forward (relative to the X-ray phase) by approximately 50 degrees. (To amplify the effect, we measure FEL power just before saturation.) We then run the gain length GUI for the original and modified $K$ values, showing an improvement by detuning the undulator Fig. 4.15.

\subsection{Conclusion}

We have presented the first gain length and taper measurements from LCLS. We find gain lengths of $\sim$ $2.9-3.3 \mathrm{~m}$ at $\lambda_{r}=1.5 \AA$, and $1.65 \mathrm{~m}$ at $\lambda_{r}=1.5 \mathrm{~nm}$. We also can more than double the coherent, FEL power over the saturation value by tapering the downstream undulators. Using the gain length measurements, we also confirm that removing an undulator to insert a self-seeding scheme will not impede normal user operations. We observe that tapering the undulators before and/or after the gap can recover some of the lost FEL power due to the gap. 


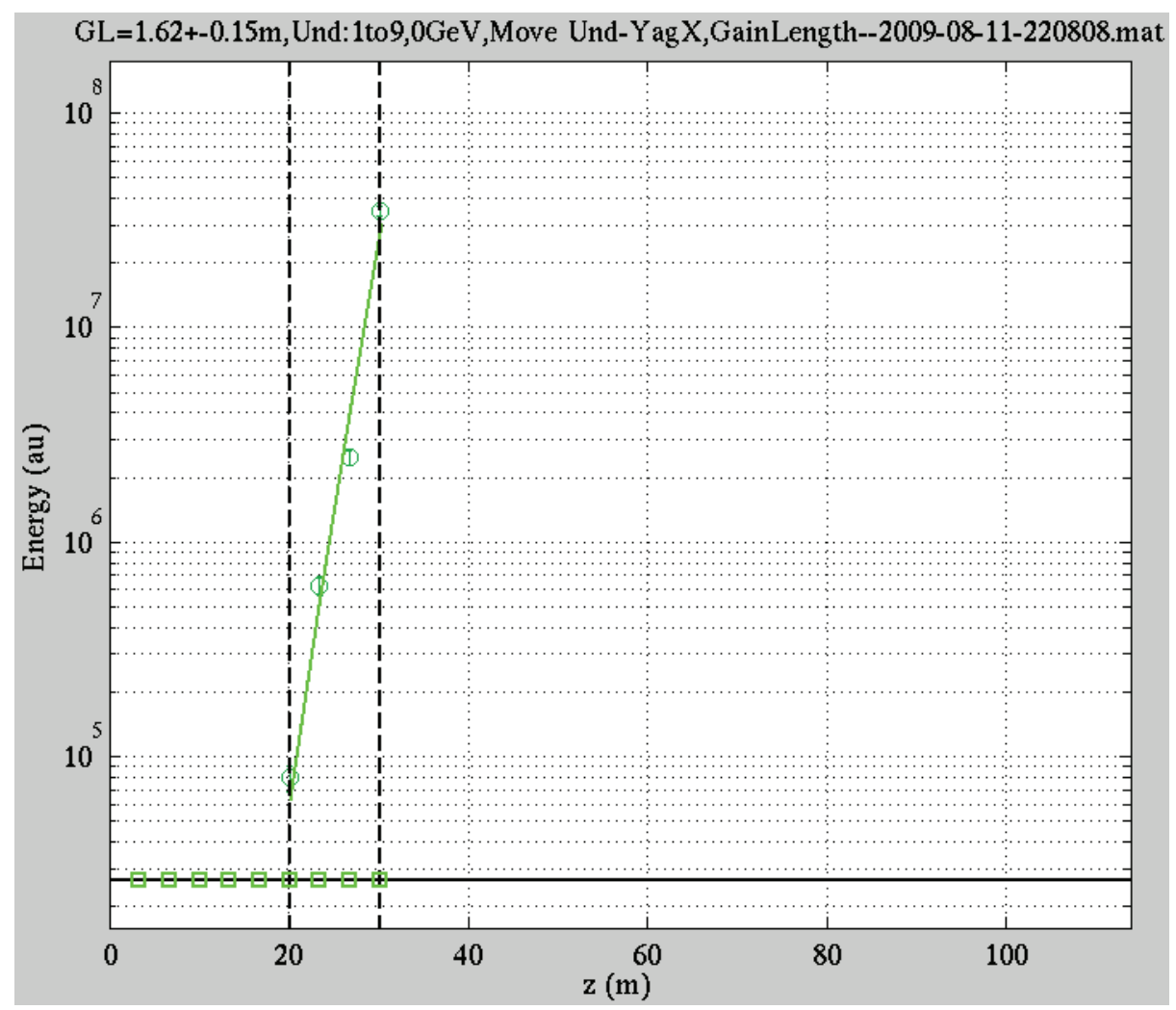

Figure 4.8: Gain length of $1.62 \pm 0.15 \mathrm{~m}$ taken at $4.7 \mathrm{GeV}$ using the undulator pull method and the YAGXRAY screen. Only four points are used due to the time required to pull each undulator and the danger of damaging YAGXRAY with high FEL power.

\subsection{Gain Length GUI}

The gain length GUI was designed as an operator tool for measuring gain length as part of routine FEL characterization. The main purpose of the GUI is measure gain lengths by recording pulse energies on a YAG screen. The GUI simultaneously measures FEL energy loss (to evaluate post-saturation performance, e.g. taper) and can determine FEL spot size, location, and jitter from the YAG measurements. A screen shot of the GUI is given in figure 4.16 .

The GUI is designed to measure the FEL gain length with a single click by an operator, but also contains a manual mode for acquiring single data points. A full measurement of all 33 undulators using the kick method takes approximately 10 minutes. For normal operation at $1.5 \AA$, measurements of about 15 undulators (taking 5 minutes) is usually sufficient to determine gain length. The undulator removal method requires three minutes to remove each undulator. All undulators are reinserted simultaneously.

The measurement can use either the kick or undulator removed method, with the undulator range specified by the user (defaulting to the full range of inserted undulators). The kick strength and direction (x or y) can be altered by the user, but defaults to $\mathrm{x}$-kicks (so as not to disturb energy loss measurements). The undulator kick method uses two kicks, separated by approximately 90 degrees in the induced oscillation. At hard X-rays, only one kick is needed for most of the measurement.

The GUI determines the location of the measurement by looking for either a missing undulator (removal 


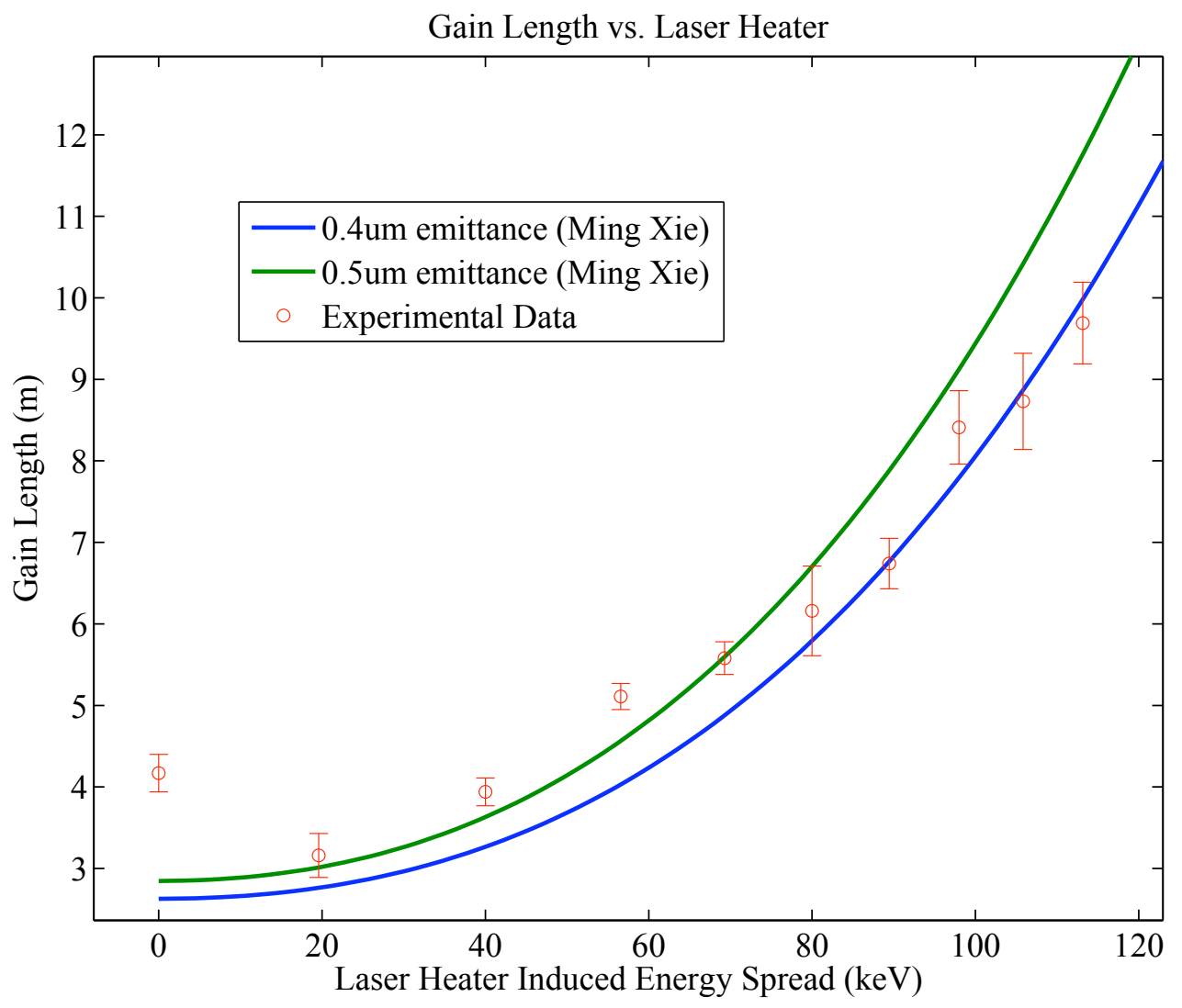

Figure 4.9: Gain length as a function of energy spread following the laser heater, with Ming Xie scaling shown for $0.4,0.5 \mu \mathrm{m}$ emittances. We note the increased gain length with no laser heating $(0 \mathrm{keV})$, hinting at the importance of the laser heater to suppress the microbunching instability. The nominal heater value induces a $20 \mathrm{keV}$ energy spread.

method) or a dipole kick. To determine dipole kicks, the starting position is assumed to be "zero" kick, since it is possible that small non-zero dipole strengths may be used to correct errors along the beam path and these should not be considered kicks. Deviations from the starting position are registered as kicks by the GUI. The user can always reset the GUI to either return to the initial configuration (kicks and undulator position) or to set the current settings as the stored "zero" configuration. The current undulator configuration is plotted beneath the measurement section, and updates following most GUI actions.

Following a measurement, all data stored in the GUI are saved whenever a plot is sent to the logbook. If no data has changed since a previous save, additional logbook plots do not trigger a save. Unlike the correlation plot GUI, it is possible to add new points to a previously saved file. In this case, sending plots to the logbook will re-save the data in a new file. Saved files can be reloaded with the gain length GUI, or analyzed with a separate file designed for printing plots for publications.

The data analysis uses Henrik Loos's profmon GUI program to calculate peak sizes from images. Paul Emma's linear fitting function then determines the gain length (by taking the log of the pulse energies). The user can select the range of points to be used for fitting (so as to avoid saturation and points below the 


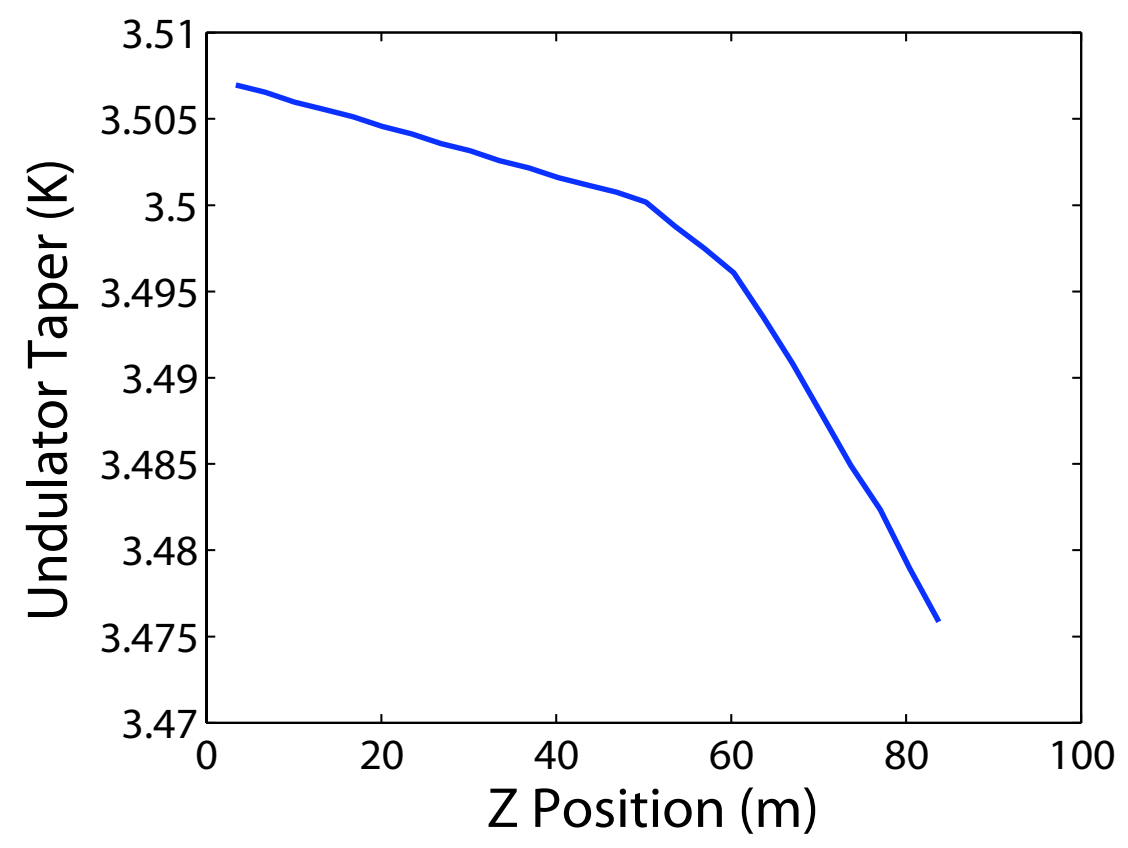

Figure 4.10: Experimentally-optimized undulator taper yielding an FEL-induced average electron energy loss of nearly $9 \mathrm{MeV}$ with the electron beam energy at $13.5 \mathrm{GeV}$. (Measurement taken on May 4th, 2009.)

noise level). The user may select between the seven analysis methods of Henrik's GUI, as well as a simple pixel sum, and may choose to plot average power, max power, or all data points. The user may also plot the FEL position $(x, y)$, position jitter $\left(\Delta_{x}, \Delta_{y}\right)$, spot size $\left(\sigma_{x}, \sigma_{y}\right)$, and energy loss (Paul Emma's E-loss measurement), all as functions of longitudinal position in the undulator line. The user must choose between YAGXRAY and the direct imager, though the GUI tries to guess which screen was used for the measurement.

The GUI changes the attenuator and camera settings automatically. If the GUI detects pulse energies that could damage the direct imager screen, it inserts solid beryllium attenuators. As the power falls, the GUI lowers the attenuation level. YAGXRAY has no attenuators, so this function works only for the direct imager. If the GUI detects camera saturation (any locally averaged group of 9 pixels near the saturation level), the GUI inserts a neutral density filter to protect the camera and avoid saturation. Both YAGXRAY and direct imager cameras have neutral density filters.

Manual modes allow the user to move dipole correctors, and remove and insert undulators individually. It is also possible to take a single data point; the GUI uses the undulator and dipole settings to determine the location of the measurement. The user can select the number of shots per measurement, as well as the strength and orientation ( $x$ vs. $y$ ) of the dipole kick. If the spontaneous background subtraction option is selected, the GUI takes an image with the FEL suppressed by a kick following the first undulator. This image should contain only spontaneous radiation (assuming no FEL restart), and is subtracted from all ensuing measurement images. If the camera filter or attenuator settings change, the GUI retakes the spontaneous image. Due to the need for extra dipole movements and images, using the spontaneous subtract slows the GUI, but is essential for use of the direct imager. It is not recommended when using YAGXRAY.

The size of the region of interest (ROI) determines the speed of YAGXRAY images and processing. The 


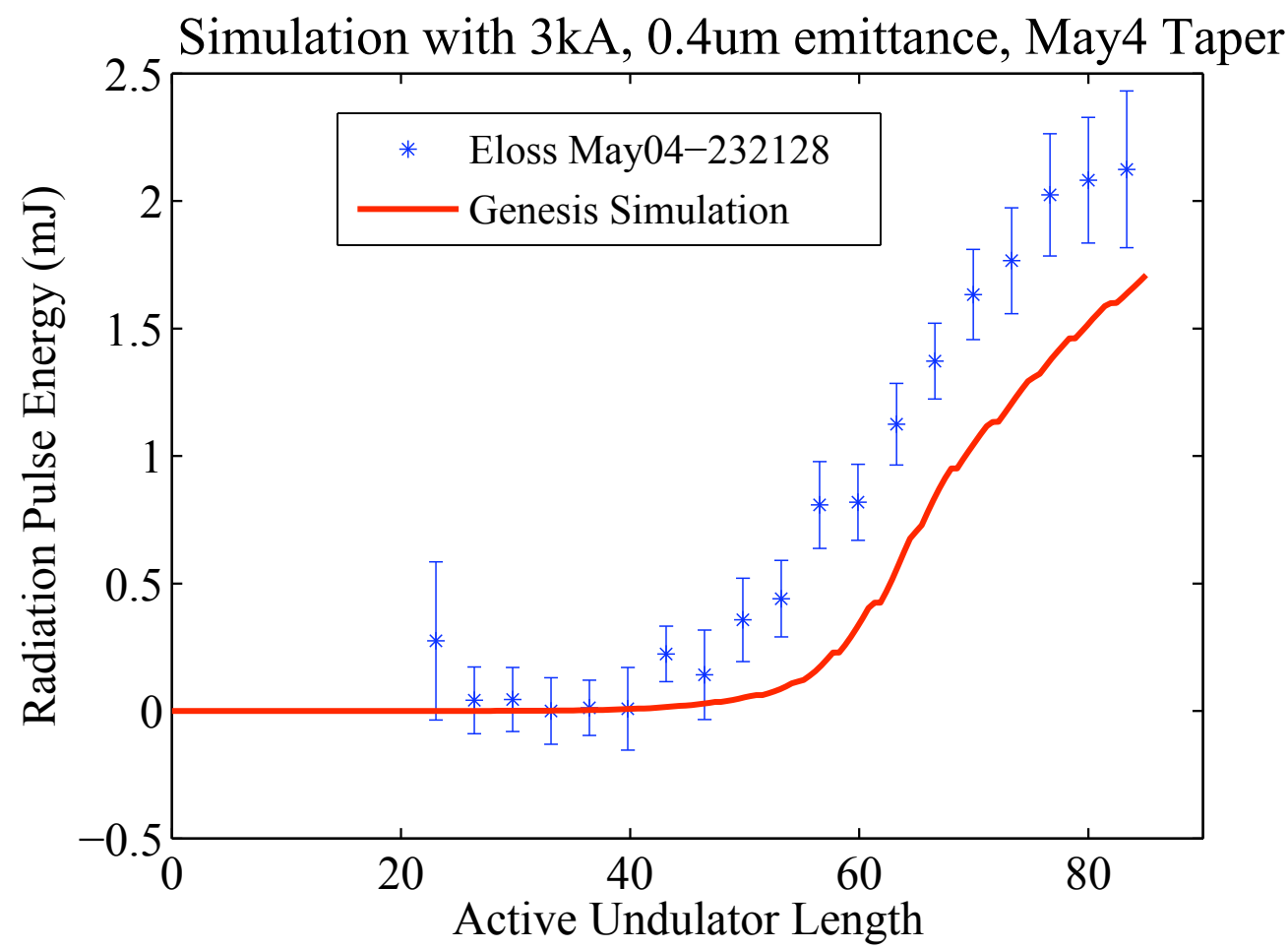

Figure 4.11: Post-saturation FEL pulse energy for a taper with nearly $9 \mathrm{MeV}$ final average electron energy loss. Genesis simulations for a $0.4 \mu \mathrm{m}$ emittance beam agree well, but have slightly lower FEL power.

user should set an ROI with the minimum size that includes the entire FEL spot, allowing some room for jitter and steering. Setting a small ROI not only speeds the measurement, but also improves results by cutting out the spontaneous background and secondary FEL spots. If the user forgets to set the ROI, the GUI will give the user an opportunity to restart. At present, it is not possible to set the ROI of the direct imager cameras through a GUI, though it can be done manually by sending pixel numbers to the camera.

The GUI stores data synchronously, so it is possible to throw out individual data points based on measured electron properties (energy, peak current, BPM positions, etc.). However, due to the unexpectedly stable operation of the FEL, this aspect is not essential to operation of the gain length measurement, and has not been used extensively. The default is off.

The GUI uses Heinz-Dieter Nuhn's Ming Xie scaling code to provide an expected gain length from the beam parameters. The Ming Xie inputs come from the most recently recorded beam parameters, or the user can also enter values manually. 


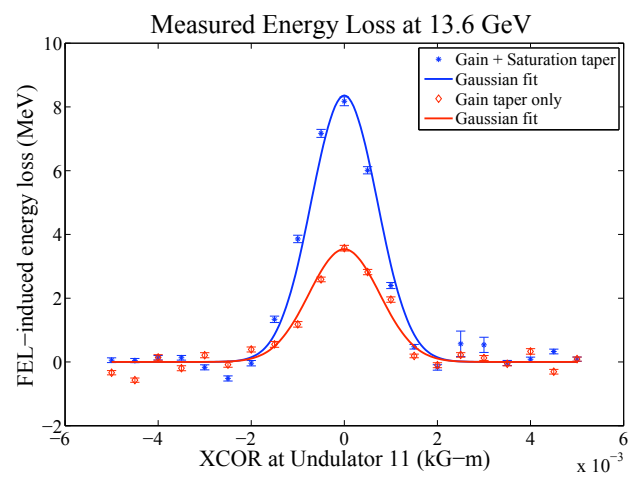

(a)

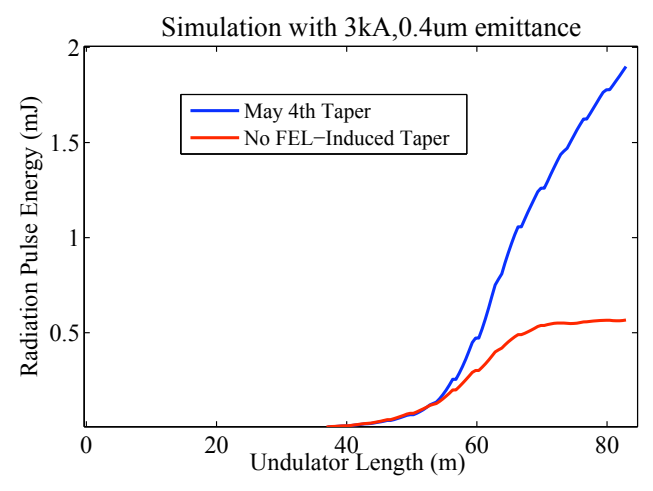

(b)

Figure 4.12: At left, measured FEL-induced electron energy loss as a function of dipole kick at undulator 11. The addition of a saturation taper can increase the FEL output by a factor of greater than 2. At right, simulation results show a post-saturation taper increasing the FEL output by greater than a factor of 3 .

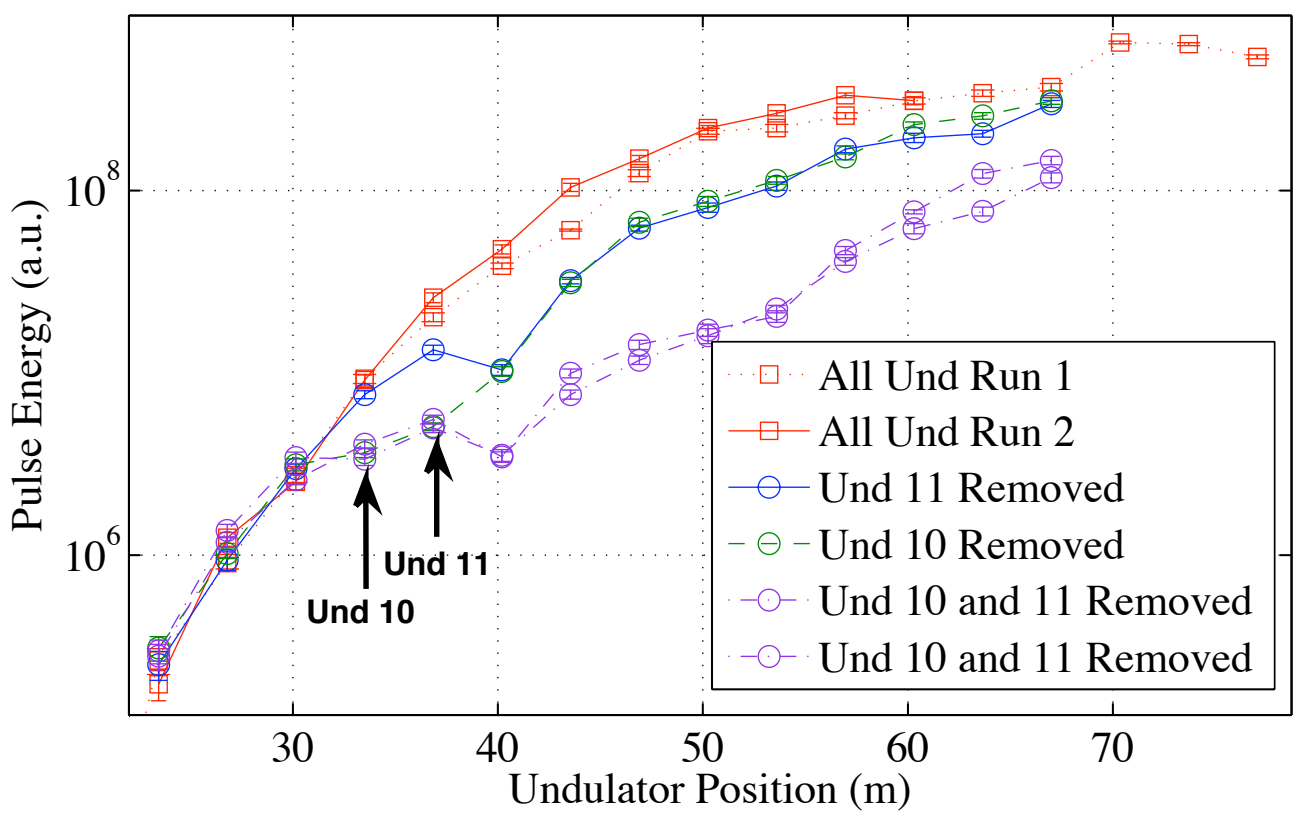

Figure 4.13: As expected, removing an undulator section suppresses the FEL growth. Without a phase error (and ignoring diffraction effects), we would expect the gap to delay the gain length curve by one section. However, we can see that removing one undulator (either 10 or 11), delays the gain curve by approximately two sections, suggesting that an additional section is required to restore the correct phase relation between electrons and X-rays. Removing two sections (10 and 11), has a much more deleterious effect. 

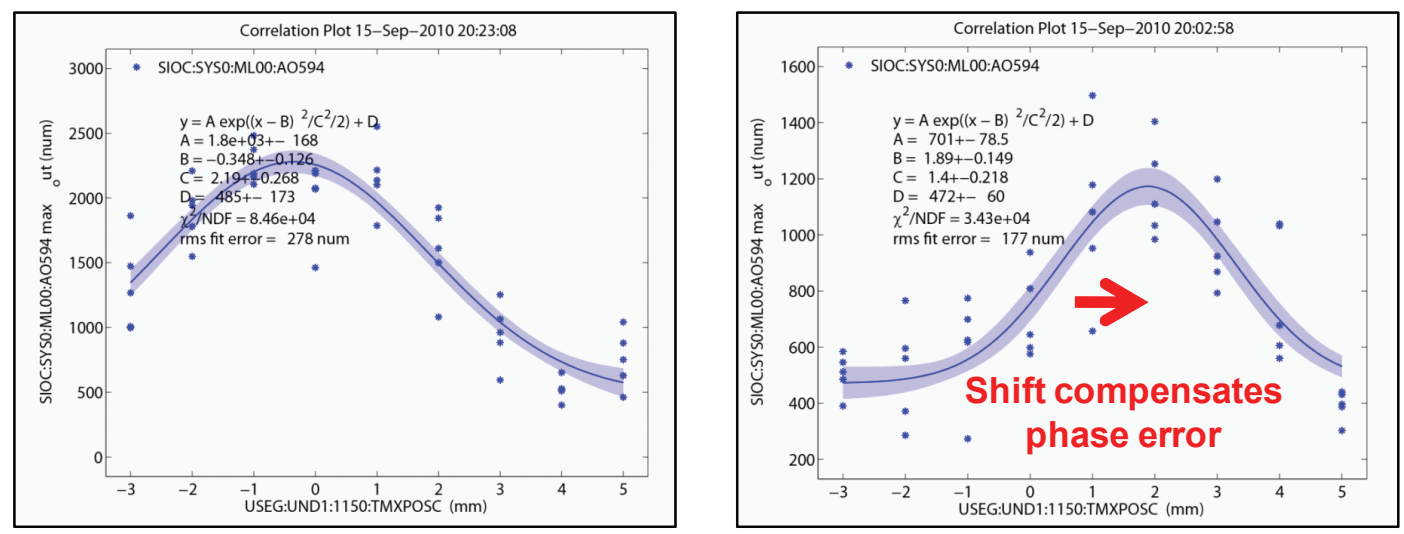

Figure 4.14: Scan of undulator $11 \mathrm{~K}$ parameter when all undulators are inserted (left) and with undulator 12 removed (right). We see that the optimal $\mathrm{K}$ parameter for undulator 11 is near the nominal value $(0 \mathrm{~mm}$ offset) when all of the undulators are inserted. However, the optimal $\mathrm{K}$ parameter shifts to smaller values when undulator 11 is followed by a gap.

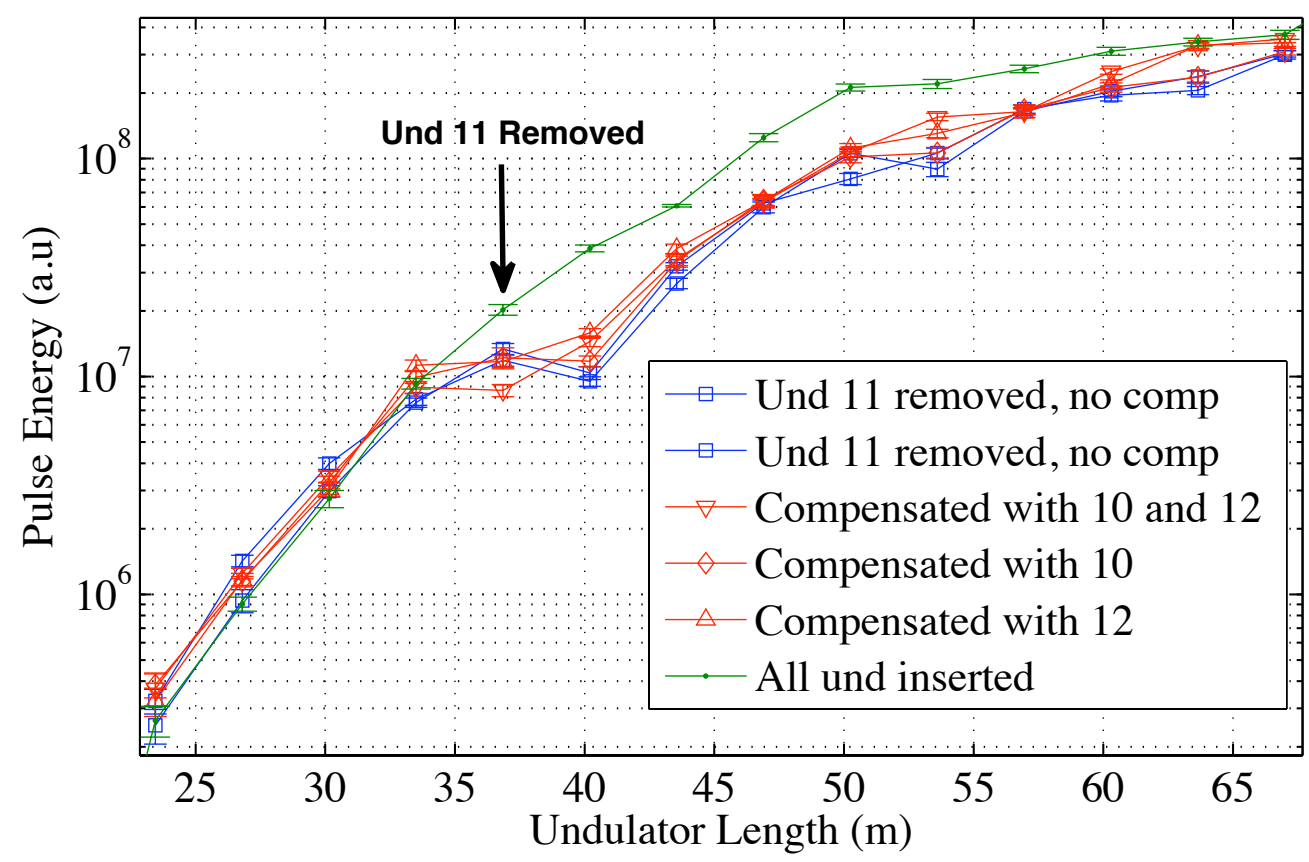

Figure 4.15: Detuning the undulators before and/or after the missing undulator partially recovers the lost FEL power. We note that a perfect phase match can at best result in a one undulator section delay. 


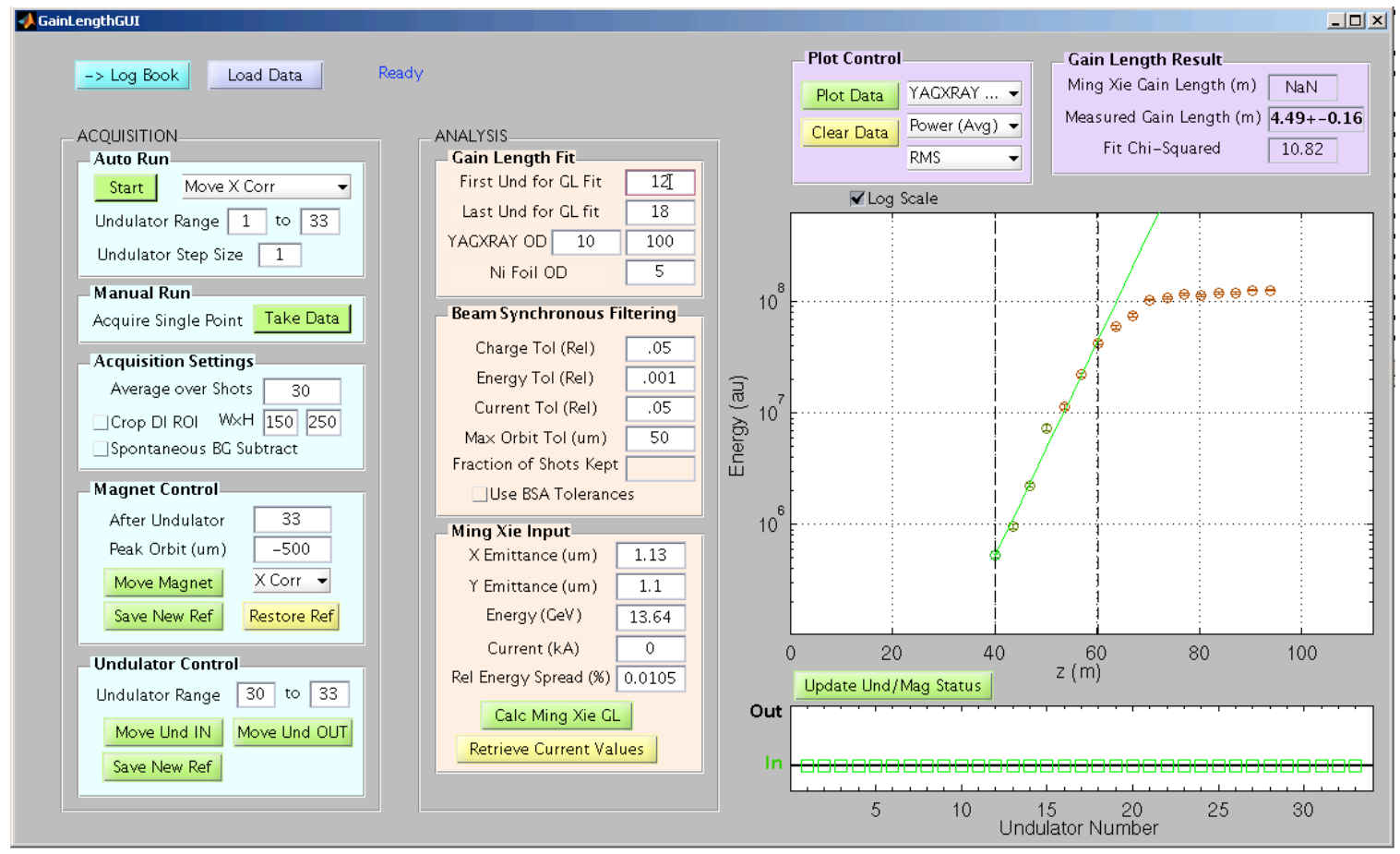

Figure 4.16: Screen-grab of the gain length GUI. 


\section{Chapter 5}

\section{Second and Third Harmonic}

\section{Measurements at the Linac Coherent}

\section{Light Source}

\subsection{Introduction}

LCLS started user commissioning in October of 2009, producing fundamental FEL radiation with photon energies ranging from $550 \mathrm{eV}$ to $9 \mathrm{keV}$ [5]. Radiation at the fundamental wavelength of the FEL dominates in the experimental beamlines, but non-negligible levels of radiation at higher harmonics are also present. These harmonics may be desirable as a source of harder x-rays, but may also contribute backgrounds to user experiments at the fundamental wavelength.

The SASE FEL interaction introduces both energy and density modulations of the electron beam at the undulator's fundamental wavelength. Close to saturation, strong bunching at this wavelength produces rich harmonic bunching as well [63]. Three-dimensional simulations [64] and analytical results [65] show that significant odd harmonic power can exist for a planar undulator at the FEL saturation. Previous short-wavelength SASE FEL experiments, such as those at LEUTL, VISA, and FLASH, have observed third-harmonic emission as high as $1 \%$ of the fundamental power $[66,67]$.

While the harmonic bunching factor is largest at the second harmonic, the symmetry of planar undulators prohibits on-axis radiation at even harmonics, so we expect the third harmonic to dominate the harmonic emission. However, due to finite electron beam size, betatron motion and radiation angle, second harmonic radiation may exist at non-negligible levels $[64,68,69]$, and has also been observed experimentally $[66,67$, 70].

In this paper we present measurements of the second and third harmonic content in the FEL at a range of photon energies. To determine the extent to which higher harmonics reach the experimental stations, we measure the photon energy cutoff of the x-ray mirrors. We also compare the results to simulations and study the effect of electron beam quality on the harmonic power. 


\subsection{Data Collection Methods}

The primary goal of this chapter is to determine the relative pulse energy levels of the harmonics in the FEL beam at LCLS. To measure the harmonic content, we separate the FEL components by photon energy. In standard operation, the sum of all higher harmonics represents at most a few percent of the FEL beam, so we take the total intensity measurements as an approximate measure of the fundamental pulse energy. We then use mirrors and attenuators to separate out the second and third harmonics, which we measure by inserting YAG screens into the $\mathrm{x}$-ray beam. We measure the third harmonic at both soft and hard $\mathrm{x}$-rays, and the second harmonic at soft x-rays only.

\subsubsection{Third Harmonic Method, Soft and Hard X-rays}

To measure the third harmonic, we attenuate the $\mathrm{x}$-ray beam with either $\mathrm{N}_{2}$ gas or solid sheets of beryllium or zirconium (Fig. 5.1). The attenuation decreases at shorter wavelengths, allowing harmonic radiation to pass while blocking the fundamental. As an example, with the fundamental set to $8 \mathrm{keV}$ photons, the zirconium filter ( $1 \mathrm{~mm}$ of silicon with $100 \mu \mathrm{m}$ of zirconium) cuts the fundamental $\mathrm{x}$-ray intensity by 10 orders of magnitude, but cuts the third harmonic by less than two orders of magnitude [3]. The attenuator does not suppress higher harmonics, but these are emitted at much lower levels in the FEL process, so we assume the radiation remaining past the attenuator is primarily third harmonic. We can also independently confirm the presence of the third harmonic by tuning the FEL to the zirconium K-edge (Section 5.3).

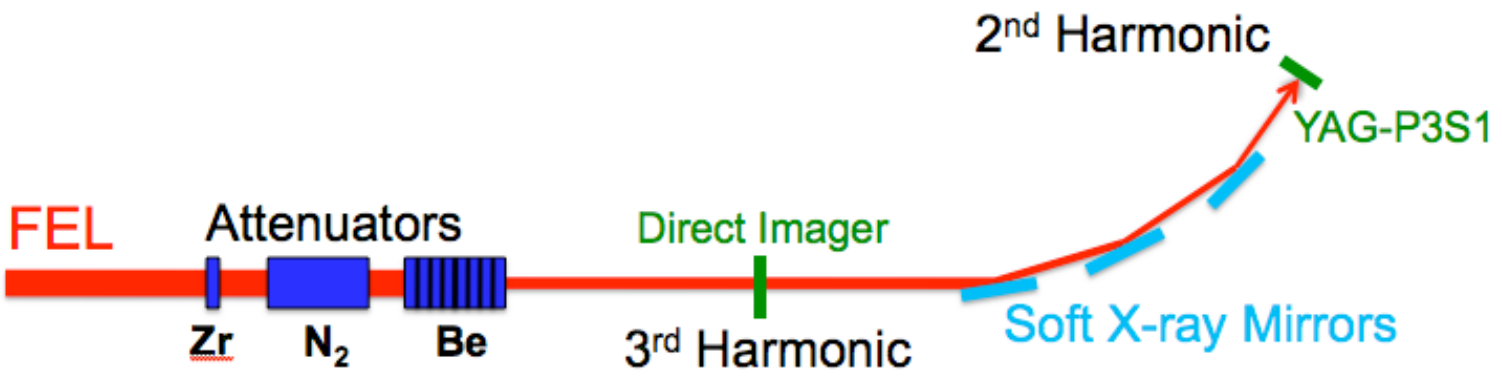

Figure 5.1: Schematic of the harmonic measurements. Attenuators block the fundamental and second harmonic, allowing measurement of the third harmonic on the Direct Imager YAG screen. Alternatively, we can isolate the second harmonic by measuring the intensity at the P3S1 YAG screen; the soft x-ray mirrors, upstream of P3S1, absorb the third harmonic. We again rely on the attenuators to block the fundamental.

\subsubsection{Second Harmonic Method, Soft X-Rays}

We measure the second harmonic, sandwiched between the stronger first and third harmonics, in the experimental beamlines. Solid or gas attenuators again block the fundamental, letting only higher harmonics pass. However, the mirrors that direct the radiation to the experimental beamlines absorb most radiation above a cutoff photon energy. By setting the FEL fundamental photon energy to between $1 / 3$ and $1 / 2$ of the cutoff, the mirrors will pass the second harmonic while absorbing the third harmonic (Fig. 5.1). With the low energy photons absorbed in the attenuators, and the high energy photons absorbed in the mirrors, only the second 
harmonic reaches the experimental beamlines. Due to the gradual energy cutoff for the hard x-ray mirrors (Fig. 5.3), the second harmonic measurements work only for soft x-rays.

\subsubsection{Intensity Measurement Methods}

We obtain x-ray intensities from either a gas detector or a YAG screen [71]. For each YAG image, we average up to 100 pulses from the FEL. Though we subtract a dark (no electron beam) background, spontaneous radiation is present in all images. To determine the FEL pulse intensity, we fit a 2-D Gaussian profile to the YAG image and calculate the volume under the FEL peak. Because the spontaneous radiation emits at a much wider angle than the FEL, we assume the spontaneous components appears as a pedestal underneath the FEL peak. We then expect the volume under the curve to represent the FEL pulse energy only.

\subsubsection{Transport Mirror Cutoff}

To determine the level of second harmonic reaching the experimental stations, we measure the photon cutoff energy of the beamline mirrors. A series of three glancing incidence mirrors diverts the x-ray beam to the soft x-ray experimental halls. The mirrors absorb hard x-ray radiation, which cannot reach the soft x-ray experimental stations. YAG screens before and after the third mirror (P2S and P3S1 respectively) measure the transmitted x-ray pulse energy. We determine the mirror cutoff energy by two methods. First, we measure the ratio of intensities on $\mathrm{P} 3 \mathrm{~S} 1$ and $\mathrm{P} 2 \mathrm{~S}$ as a function of photon energy. Assuming all three mirrors are identical, we plot the cube of this ratio as the total transmission of the mirrors. However, the transmission of each stage may differ, for example if the mirror aperture cuts a portion of the beam. As a semi-independent method, we also compare the signal on P3S1 to the total incoming power measured in the gas detectors, located upstream of the mirrors. Fig. 5.2 diagrams both methods, and we plot the results in Fig. 5.3, showing a cutoff energy of approximately $2.3 \mathrm{keV}$ as expected [2].

A set of two mirrors directs high energy radiation to the hard x-ray hutches. The photon energy cutoff of the hard x-ray line is far beyond the limit of the fundamental FEL, so we insert an attenuator that allows only third harmonic radiation to pass. We again vary the photon energy while comparing the third harmonic power seen before and after the final mirror (using screens $\mathrm{P} 2 \mathrm{H}$ and $\mathrm{P} 3 \mathrm{H}$ respectively). We compare the measured and expected values in Fig. 5.3 [72]. The discrepancy between the curves is likely the result of a change in the angle of the mirrors, which must move to redirect the beam to the far experimental hall. With a cutoff approximately a factor of three above the maximum energy of the fundamental, we expect all of the second harmonic to reach the hard $\mathrm{x}$-ray experimental stations. Third harmonic radiation up to $25 \mathrm{keV}$ will also pass through to the hard $\mathrm{x}$-ray lines.

\subsection{Third Harmonic Measurements, Soft and Hard X-rays}

\subsubsection{Attenuator Scan}

To measure the third harmonic, we block the fundamental with either beryllium or zirconium attenuators. (The attenuation also blocks the weaker second harmonic.) To find the relative power of the third harmonic, we can simply take the ratio of intensities on the YAG screen with a sufficiently thick attenuator inserted 


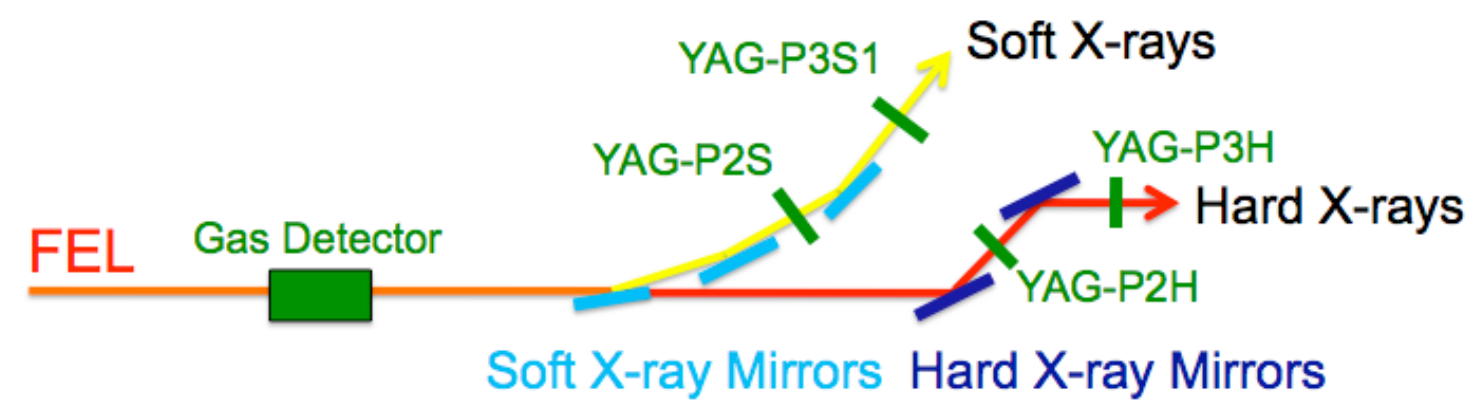

Figure 5.2: Schematic of the mirror cutoff measurement. For the soft $\mathrm{x}$-ray line, the ratio of intensities measured on YAG screens P3S1 and P2S gives the transmission for a single mirror, and the total transmission is assumed to be the cube of this ratio. Alternatively, we can compare the intensity on P3S1 (downstream of all mirrors) directly against the intensity at the gas detector (upstream of all mirrors). For the hard x-ray line, attenuators block the fundamental, and we calculate the square of the ratio of third harmonic measured before $(\mathrm{P} 3 \mathrm{H})$ and after $(\mathrm{P} 2 \mathrm{H})$ the second mirror.

(primarily third harmonic) and removed (primarily fundamental). For $0.9 \mathrm{keV}$ and $1.7 \mathrm{keV}$ fundamental photons, we find approximately $2 \%$ and $3 \%$ harmonic content respectively.

We can also vary the level of attenuation, and fit the harmonic content to the resulting curve. By measuring the transmission values for the filters, we simultaneously confirm the validity of the harmonic measurement. The total intensity is proportional to

$$
I(d) \propto T_{1}(d) P_{1}+T_{2}(d) P_{2}+T_{3}(d) P_{3}+\text { higher harmonics }
$$

where, for harmonic $h$, we have attenuator transmission, $T_{h}(d)$, and FEL power $P_{h}$. We assume that the transmission can be described by a simple exponential,

$$
T_{h}(d)=e^{-d / \Lambda_{h}}
$$

with the attenuation length, $\Lambda_{h}$, determined by the attenuator composition, the fundamental photon energy, and the harmonic of interest. The attenuator parameter, $d$, can be either the thickness of a solid attenuator (units of $\mathrm{mm}$ ) or the pressure in a gas attenuator (units of torr). The attenuation length, $\Lambda_{h}$, then has units of either $\mathrm{mm}$ for the solid attenuator or torr for the gas attenuator. Neglecting the weaker second and higher harmonics, we expect

$$
I(d) \propto e^{-d / \Lambda_{1}}+e^{-d / \Lambda_{3}} \frac{P_{3}}{P_{1}} .
$$

Measuring the intensity, $I(d)$, for a range of $d$, we can then find the ratio of the harmonics, $P_{3} / P_{1}$, from a one-parameter, linear fit.

The beryllium filter transmission is difficult to estimate from the composition of the filters; even low levels of impurities of heavy elements can have a large impact on the absorption of hard x-rays. To confirm the transmission values, we repeat the analysis with a three-parameter nonlinear fit of both attenuation lengths, $\Lambda_{1}, \Lambda_{3}$ as well as the harmonic ratio, $P_{3} / P_{1}$. For data sets without multiple measurements per attenuator setting, we assume a measurement error of $5 \%$ for each data point. To estimate error bars for the fit, we 

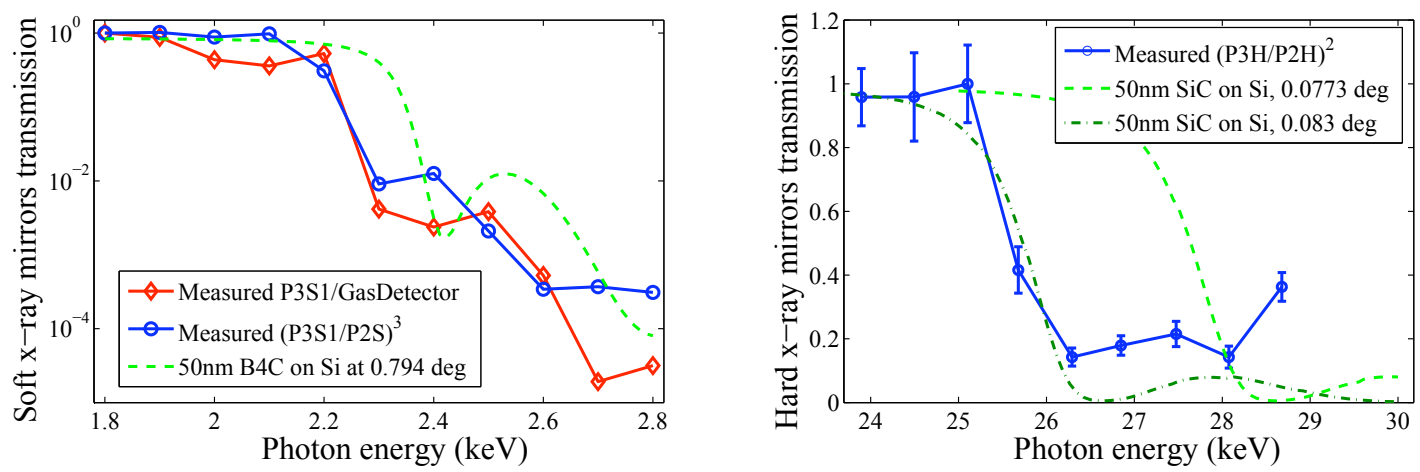

Figure 5.3: Transmission plots show the photon energy cutoffs of the soft x-ray (left plot) and hard x-ray (right plot) experimental beamlines. On the left, blue circles show the cube of the ratio of intensities on P3S1 and P2S, while the red diamonds give the ratio from P3S1 to the full pulse energy. Both results match the expected transmission (dashed green curve) [2,3]. On the right, blue circles give the square of the ratio of intensities measured on $\mathrm{P} 3 \mathrm{H}$ and $\mathrm{P} 2 \mathrm{H}$. The dashed curve gives the expected transmission at the nominal 0.0773 degree angle; however, we find a better match for 0.083 degree angle (dot-dash curve). Hard x-ray measurements rely on the third harmonic, resulting in lower resolution than for the soft x-ray measurements. In both cases, the experimental curves are normalized to one at the maximal value.

perform a monte carlo simulation, randomizing each data point according to its error and repeating the fit. We find the attenuation lengths at the fundamental, $\Lambda_{1}$, match the expected values reasonably well (Table 5.1). Due to the long attenuation lengths for the third harmonic at high photon energies, the errors on $\Lambda_{3}$ are large. (However, we note that the harmonic ratio, $P_{3} / P_{1}$, is not sensitive to $\Lambda_{3}$.)

At lower photon energies, we assume the YAG response to the fundamental and third harmonic is equivalent. However, as the photon energy increases, the YAG screen may not fully absorb the third harmonic. For the $6 \mathrm{keV}$ fundamental measurement, a $100 \mu \mathrm{m}$ YAG largely absorbs the third harmonic due to the yttrium $\mathrm{K}$-edge at $17 \mathrm{keV}$. At $8 \mathrm{keV}$ fundamental, approximately $40 \%$ of the third harmonic passes through the 100 $\mu \mathrm{m}$ YAG, so we use a $1 \mathrm{~mm}$ YAG for this measurement.

Fig. 5.4 shows several sample measurements, with $0.8-2 \%$ third harmonic content at a range of photon energies. We generally find slightly higher harmonic content with the fundamental set to longer wavelengths, but the harmonic content at any particular wavelength may vary widely depending on the current beam properties.

\subsubsection{K-edge Scan}

With the FEL fundamental photon energy tuned to $6 \mathrm{keV}$, we can confirm the third harmonic measurement by inserting a zirconium filter and scanning the FEL photon energy around the K-edge (Fig. 5.5). A small shift in energy of a few percent has little effect on the FEL performance, but changes the zirconium transmission at $18 \mathrm{keV}$ by more than two orders of magnitude; we conclude that any drop in measured intensity must come from radiation at the $18 \mathrm{keV} \mathrm{K-edge.} \mathrm{With} \mathrm{the} \mathrm{zirconium} \mathrm{filter} \mathrm{inserted,} \mathrm{the} \mathrm{intensity} \mathrm{drops} \mathrm{by} \mathrm{more} \mathrm{than} \mathrm{a}$ factor of 40 across the K-edge (Fig. 5.6), so we conclude that below the K-edge the measured signal consists almost entirely of third harmonic. 

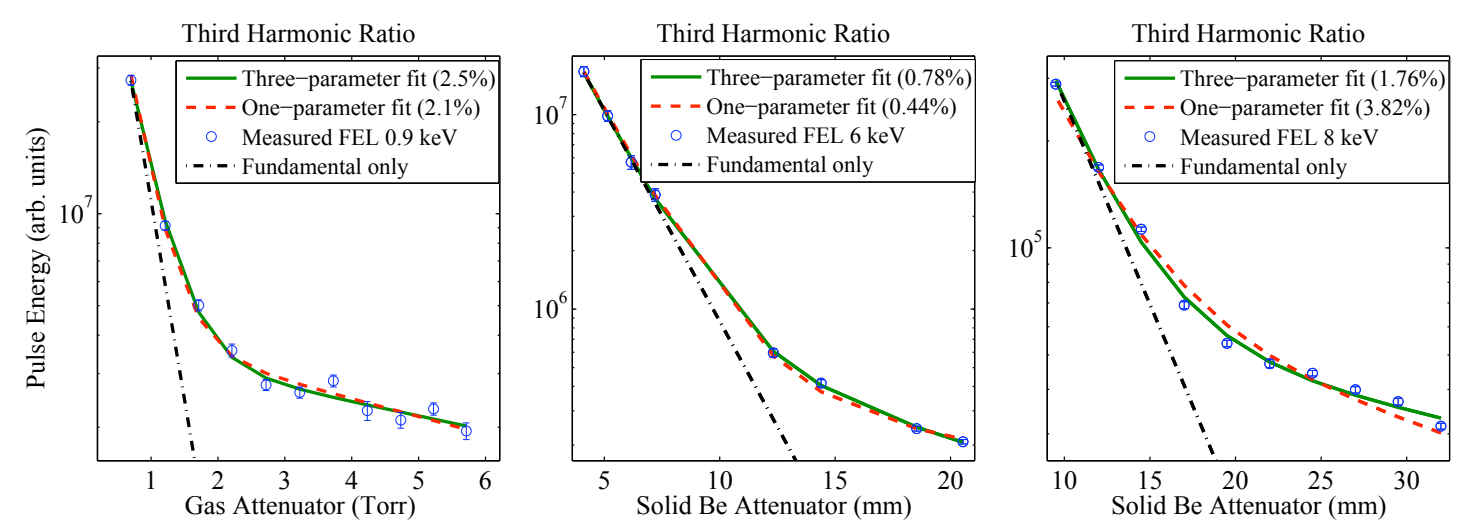

Figure 5.4: Examples of third harmonic measurements for fundamental FEL photon energies of $0.9 \mathrm{keV}$ (left), $6 \mathrm{keV}$ (center) and $8 \mathrm{keV}$ (right). Blue circles show the experimental data, red dotted lines show linear one-parameter fits using the nominal attenuation lengths, while solid green lines show three-parameter fits to both the harmonic content and attenuation lengths. The black dot-dash line shows the expected drop in intensity for the fundamental only. We typically find around $2 \%$ third harmonic for soft x-rays, with between 0.2-2\% third harmonic at hard x-rays. The $4 \%$ level found from the one-parameter fit at $8 \mathrm{keV}$ (right plot) is higher than expected, and may be artificially high due to a lack of measurement points dominated by the fundamental.

To determine the third harmonic content, we set the third harmonic photon energy $300 \mathrm{eV}$ below the K-edge, and compare the signal with the zirconium filter inserted (primarily third harmonic) and removed (combination of third harmonic and fundamental). To prevent YAG and camera saturation during the fundamental measurement, we insert a $4 \mathrm{~mm} \mathrm{Be}$ filter. We can then find the harmonic content from the ratio of the beryllium $\left(I^{(\mathrm{Be})}\right)$ and zirconium $\left(I^{(\mathrm{Zr})}\right)$ signals

$$
\frac{I^{(\mathrm{Be})}}{I^{(\mathrm{Zr})}}=\frac{T_{1}^{(\mathrm{Be})} P_{1}+T_{3}^{(\mathrm{Be})} P_{3}}{T_{1}^{(\mathrm{Zr})} P_{1}+T_{3}^{(\mathrm{Zr})} P_{3}} \approx \frac{T_{1}^{(\mathrm{Be})}}{T_{3}^{(\mathrm{Zr})}} \frac{P_{1}}{P_{3}}+\frac{T_{3}^{(\mathrm{Be})}}{T_{3}^{(\mathrm{Zr})}},
$$

with power, $P_{h}$, and transmission factors, $T_{h}$, for both the zirconium and beryllium attenuators at harmonic, $h$. Plugging in the known transmission values (the beryllium filter transmits $8 \%$ fundamental and nearly $100 \%$ third harmonic), we find approximately $2 \%$ third harmonic content, confirming the results of Fig. 5.4.

\begin{tabular}{|c|c|c|}
\hline Photon Energy & Nominal $\Lambda_{1}, \Lambda_{3}$ & Nonlinear Fit $\Lambda_{1}, \Lambda_{3}$ \\
\hline $0.9 \mathrm{keV}$ & $0.33,7.42 \mathrm{torr}$ & $0.38 \pm 0.02,9.4 \pm 1.8 \mathrm{torr}$ \\
$6 \mathrm{keV}$ & $2.0,21 \mathrm{~mm}$ & $1.9 \pm 0.1,12.5 \pm 3.3 \mathrm{~mm}$ \\
$8 \mathrm{keV}$ & $3.8,28 \mathrm{~mm}$ & $3.33 \pm 0.30,-\mathrm{mm}$ \\
$9 \mathrm{keV}$ & $5.1,30 \mathrm{~mm}$ & $5.2 \pm 0.1,-\mathrm{mm}$ \\
\hline
\end{tabular}

Table 5.1: Nominal (middle column) and measured (right column) attenuation lengths for the fundamental and third harmonic at three different photon energies. The nominal and fitted attenuation lengths match well for the fundamental, but in general the fitted third harmonic values are lower than expected. Fitting for the the third harmonic attenuation length at hard x-rays is not effective due to the low absorption of the third harmonic. 


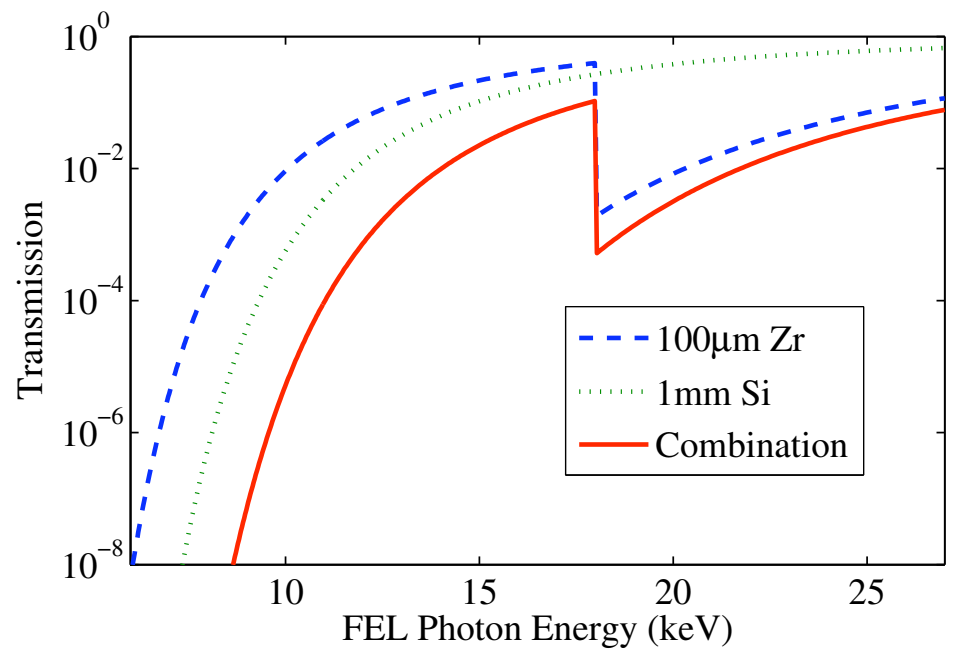

Figure 5.5: Transmission for the zirconium filter as a function of photon energy. The filter is made of $100 \mu \mathrm{m}$ of zirconium (dashed blue) and $1 \mathrm{~mm}$ silicon (dotted green), with the combined transmission given in solid red. The zirconium K-edge manifests as a sharp drop in transmission at $18 \mathrm{keV}$.

\subsection{Second Harmonic Measurements, Soft X-rays}

We expect weaker second harmonic than third harmonic due to the symmetry of the planar LCLS undulators. Though on-axis radiation is suppressed at even harmonics, the finite beam size and betatron oscillations lead to non-negligible second harmonic content $[64,68,69]$. (We note that the bunching factor, a measure of the longitudinal correlation of particles, is stronger for the second harmonic. Tuning a group of 'afterburner' undulators to double the resonant frequency can exploit this bunching to produce even more power at the second harmonic than would otherwise exist at the third harmonic [73]. In this chapter we assume the undulators are tuned to the fundamental.) By measuring the beam intensity following the soft x-ray mirrors, we are able to isolate the second harmonic. An example image (Fig. 5.7) shows the characteristic double lobe structure expected for the second harmonic (e.g. [69]).

To measure the second harmonic component we again vary the attenuation and fit the ratio $P_{2} / P_{1}$ from the intensity

$$
\begin{aligned}
I & \propto e^{-d / \Lambda_{1}} M_{1}^{3}+e^{-d / \Lambda_{2}} M_{2}^{3} \frac{P_{2}}{P_{1}}+e^{-d / \Lambda_{3}} M_{3}^{3} \frac{P_{3}}{P_{1}} \\
& \approx e^{-d / \Lambda_{1}}+e^{-d / \Lambda_{2}} \frac{P_{2}}{P_{1}},
\end{aligned}
$$

where we have assumed the mirror transmission, $M_{h}$ is perfect for the fundamental and second harmonic, and zero for the third harmonic (Fig. 5.3).

The second harmonic measurements are especially sensitive to the $N_{2}$ transmission value at the fundamental. When the attenuation is strongest, the harmonic dominates and the attenuation length at the fundamental has little effect. However, at the lowest attenuation levels when the fundamental dominates, the $\mathrm{N}_{2}$ still provides as many as six attenuation lengths at the fundamental. As a result, even small errors in the attenuation length lead to large errors in the measured power level. (The need for heavy attenuation is due to the small dynamic range of the P3S1 camera, which was not intended for this use.) 


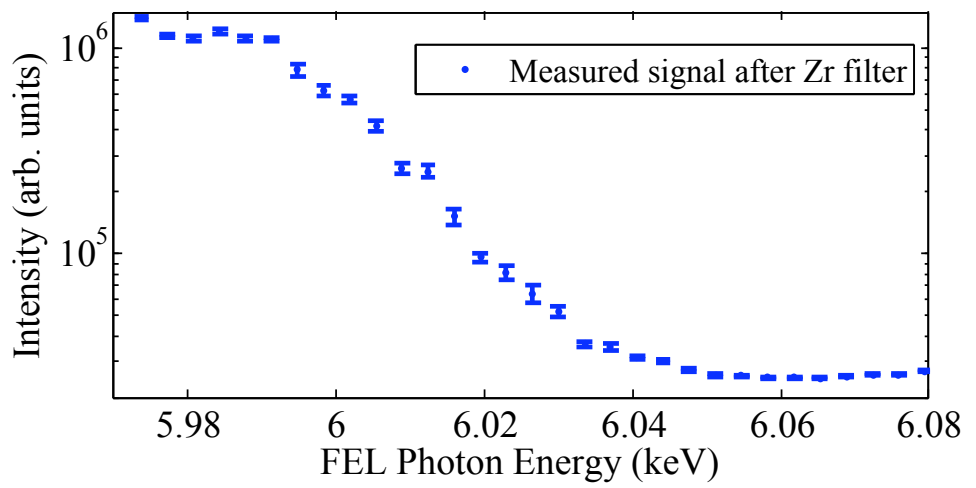

Figure 5.6: Plot of measured intensity on the Direct Imager YAG screen (Y-axis) vs. photon energy (Xaxis), with the FEL tuned to near $6 \mathrm{keV}$ fundamental and the zirconium attenuator inserted. The drop-off in intensity as the fundamental photon energy crosses $6 \mathrm{keV}$ corresponds to the zirconium K-edge at $18 \mathrm{keV}$; we conclude that the drop in intensity is due to third harmonic content. With the zirconium filter blocking the fundamental, we find that the remaining radiation is almost entirely third harmonic. Electron energy jitter and FEL bandwidth broaden the otherwise sharp K-edge seen in Fig. 5.5.

To confirm the attenuation lengths, we again fit the measured intensities to two generic exponentials. The measured and nominal attenuation lengths match reasonably well at the fundamental, but differ substantially at the second harmonic (Table 5.2). Fig. 5.8 gives sample results for the second harmonic content.

\begin{tabular}{|c|c|c|}
\hline Photon Energy & Nominal $\Lambda_{1}, \Lambda_{2}$ & Nonlinear Fit $\Lambda_{1}, \Lambda_{2}$ \\
\hline $0.9 \mathrm{keV}$ & $0.31,2.15$ torr & $0.28 \pm 0.02,1.54 \pm 0.08$ torr \\
$1 \mathrm{keV}$ & $0.42,2.95$ torr & $0.44 \pm .07,2.27 \pm .47$ torr \\
\hline
\end{tabular}

Table 5.2: Nominal (middle column) and measured (right column) second harmonic attenuation lengths for the gas detector. The nominal and fitted attenuation lengths match well for the fundamental, but in general the fitted second harmonic values are lower than expected.

\subsection{Genesis Simulations}

We compare our results to simulations from the 3D wiggler-averaged code Genesis [58], using the parameters of Table 7.1. Simulations at FEL saturation predict approximately $3 \%$ third harmonic at soft x-rays (830 eV in simulation), and approximately $2 \%$ third harmonic at hard $\mathrm{x}$-rays ( $8.3 \mathrm{keV}$ in simulation). For both energies, the fifth harmonic is approximately an order of magnitude weaker in simulations, and thus could contribute slightly to the third harmonic measurements. (We have not tried to separate the third harmonic from the higher harmonic contributions.) We conclude that the measured third harmonic content is approximately consistent with the level expected from theory and simulations, albeit with weaker harmonics than expected at high energies [65, 74]. Second harmonic levels from Genesis are also in approximate agreement with results. However, Genesis is a wiggler-averaged code (the beam centroid does not move off-axis), so comparisons with second harmonic simulations require further study. 


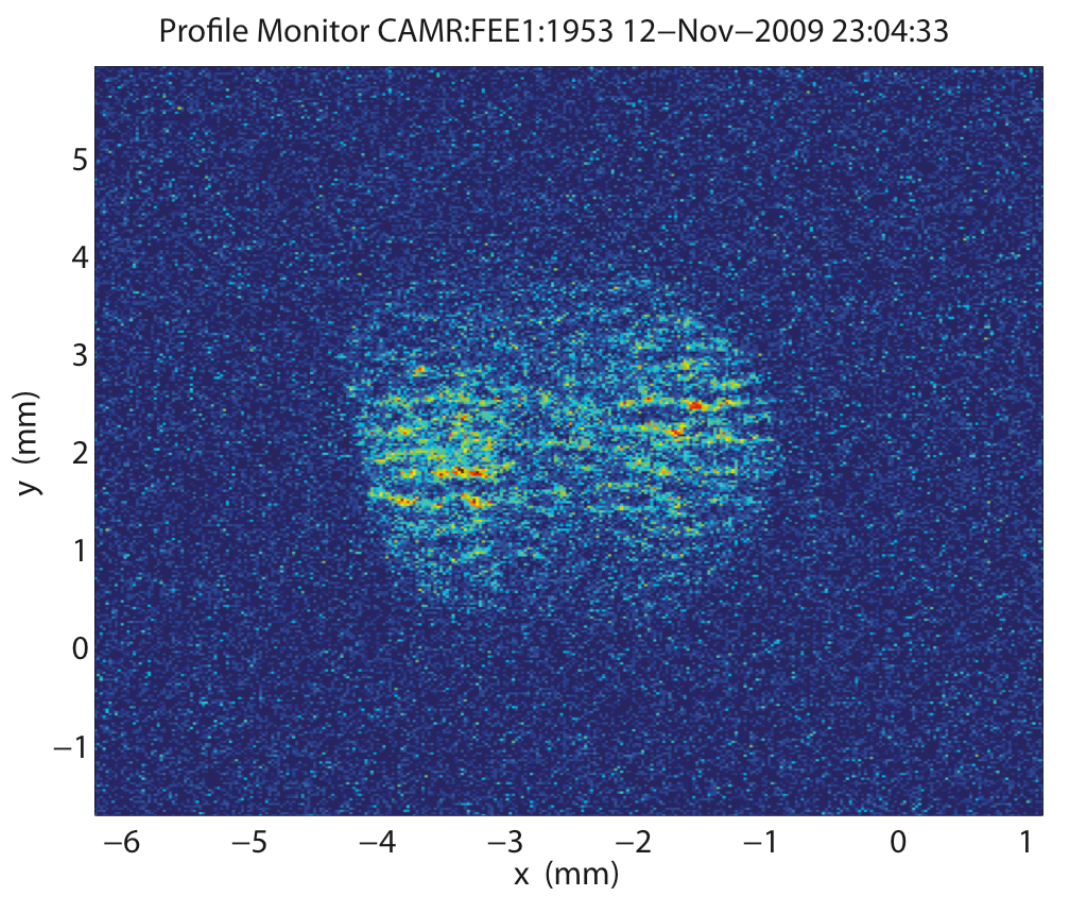

Figure 5.7: An example image in the soft x-ray beam line (P3S1) shows the characteristic double lobe structure of the second harmonic. Though gas and solid (beryllium) attenuators strongly suppress the fundamental in this image, a small amount of fundamental radiation remains (gaussian mode background). Diffraction from the beryllium attenuators produces the uneven speckle pattern.

\subsection{Sensitivity to Beam Quality}

We expect the proportion of harmonics present to vary depending on the performance of the fundamental. The lowest and highest harmonic contents in Fig. 5.4 were measured with $0.6 \mathrm{~mJ}$ and $1.5 \mathrm{~mJ}$ fundamental pulse energy respectively. Due to the increased sensitivity to electron bunch quality at shorter wavelengths, the correlation between low fundamental pulse energy and low harmonic content is not surprising. (Low fundamental energy may indicate decreased bunching factor at the fundamental, which would have a larger effect on the higher harmonics.)

To test the sensitivity of harmonics to beam quality, we repeat the third harmonic measurements while intentionally degrading the beam quality. A convenient method for changing the beam quality is the laser heater, which can increase the electron energy spread in a simple and measurable fashion. The laser heater interacts with the beam in an undulator. By placing the undulator in the middle of a chicane, the sinusoidal energy modulation washes out into an approximately uniform increase in energy spread [4]. The energy spread increases as the square root of the laser power, allowing us to triple the energy spread in the beam (Fig. 5.9). (At low laser power, a trickle heating causes a noticeable bump in the energy spread above the expected level [4]. The trickle heating is due to an instability resulting from surviving energy modulation downstream from the heater, a process analogous to the microbunching instability of chapter 3 ).

We then measure the $8 \mathrm{keV}$ third harmonic content at each of the four laser heater strengths. To ensure that we operate in the saturation region, we insert all 28 available undulators for each measurement and confirm 

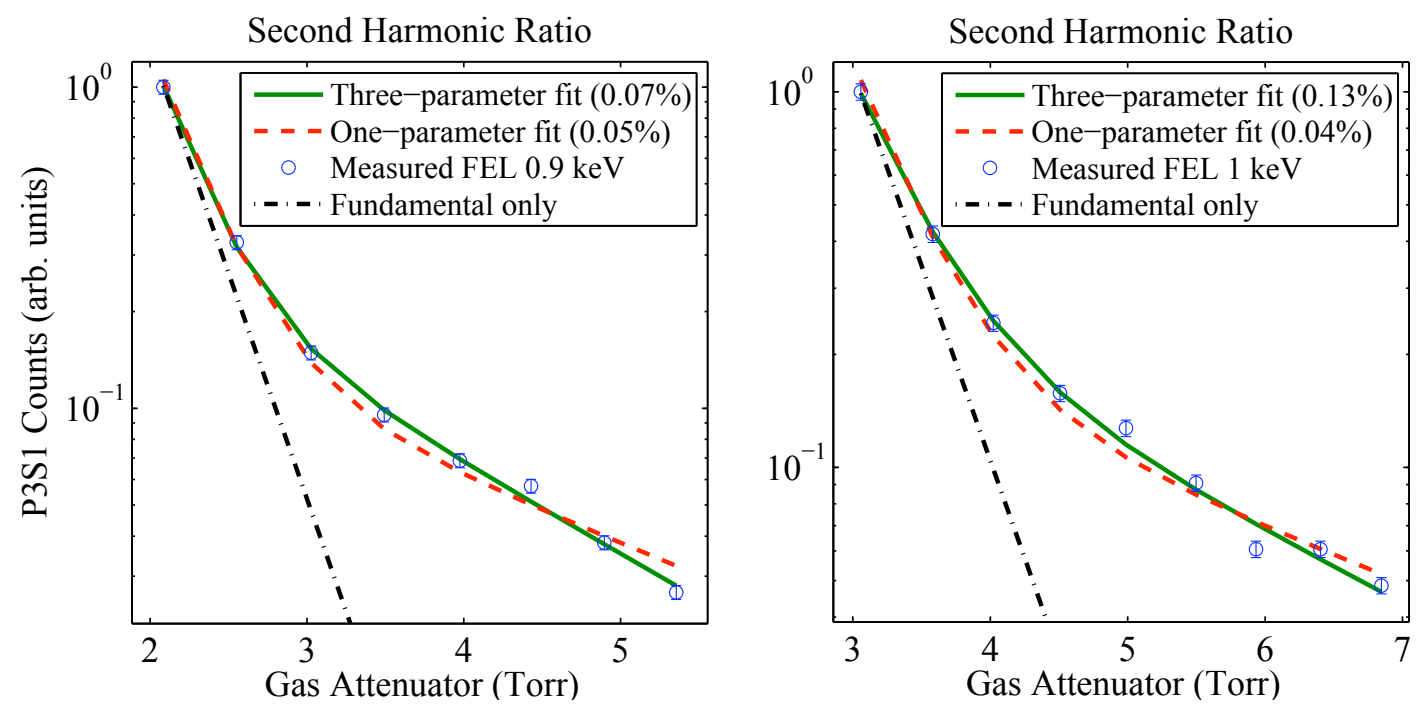

Figure 5.8: We measure the harmonic component by varying the gas attenuator strength for $0.9 \mathrm{keV}$ (left) and $1 \mathrm{keV}$ (right) fundamental photon energies. Blue circles show experimental data, red dotted lines show one-parameter fits using the nominal attenuation lengths, and solid green lines show three-parameter fits to both the harmonic content and attenuation lengths. The black dot-dash line shows the expected attenuation of the fundamental only. We find approximately $0.05-0.1 \%$ second harmonic content.

that we reach saturation [56]. We find that as the FEL performance drops (determined by the total power in the FEL beam), the proportion of power due to the third harmonic also drops (Fig 5.10). By increasing the laser heater above the nominal setting, users may be able to suppress unwanted harmonics.

\subsection{Conclusion}

We present second and third harmonic measurements for LCLS. At low energies (below $1 \mathrm{keV}$ fundamental) we measure less than $0.1 \%$ second harmonic content. The second harmonic reaches the soft $\mathrm{x}$-ray beam line for fundamental photon energies below approximately $1.15 \mathrm{keV}$ (cutoff around $2.3 \mathrm{keV}$ ). At low and high energies, we measure third harmonic content ranging from $0.2 \%$ to $2.5 \%$, which is consistent with

\begin{tabular}{|c|c|c|}
\hline & Soft X-Rays & Hard X-Rays \\
\hline Photon energy & $0.83 \mathrm{keV}$ & $8.3 \mathrm{keV}$ \\
Electron energy & $4.3 \mathrm{GeV}$ & $13.6 \mathrm{GeV}$ \\
Emit. (x,y, norm.) & $0.4 \mu \mathrm{m}$ & $0.4 \mu \mathrm{m}$ \\
Peak current & $1 \mathrm{kA}$ & $1 \mathrm{kA}$ \\
Energy spread & $3 \times 10^{-4}$ & $1 \times 10^{-4}$ \\
Third Harmonic Content & $3 \%$ & $2 \%$ \\
\hline
\end{tabular}

Table 5.3: Genesis simulation parameters given for soft and hard x-ray simulations. The simulations are consistent with measurements for both soft and hard x-rays. 


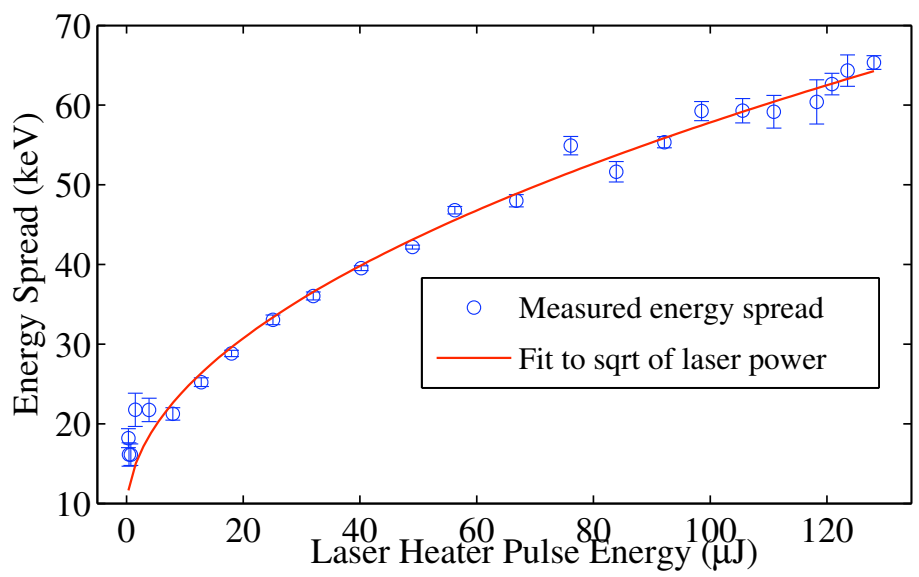

Figure 5.9: Energy spread measured as a function of laser heater power. To determine energy spread, we measure the beam width in the dispersive region a few meters downstream from the laser heater. The energy spread in keV is approximately $\Delta E=8 \sqrt{P_{L}}$, with the laser heater power, $P_{L}$, given in $\mu \mathrm{J}$ [4]. Note that at low laser power trickle heating causes a noticeable bump in the energy spread.

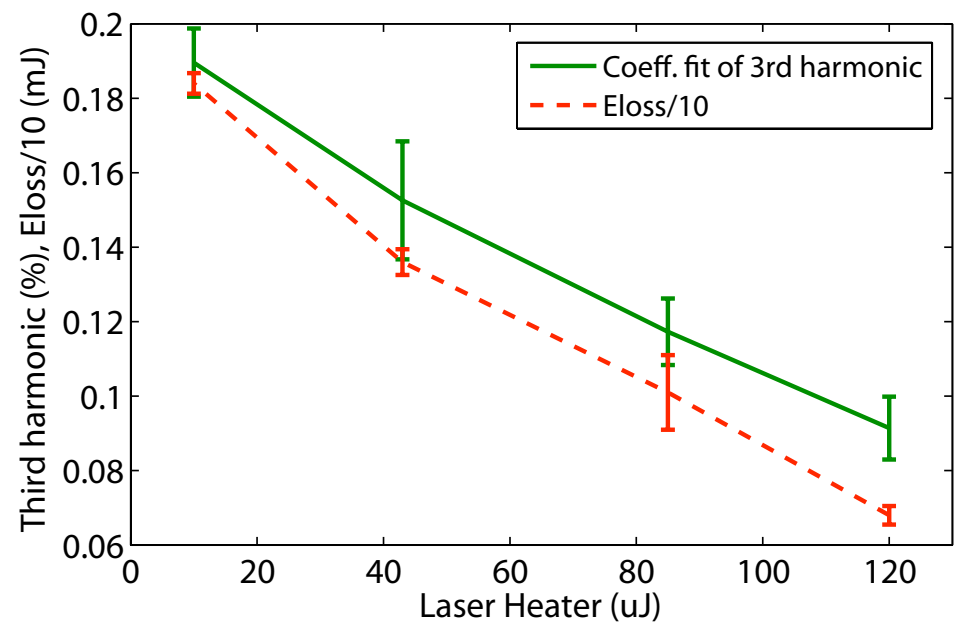

Figure 5.10: Percentage of harmonic power vs. laser heater setting. As the FEL performance drops (dotted red line), the percentage of third harmonic also drops (solid green), indicating enhanced sensitivity to beam quality at higher harmonics. The FEL performance was determined by energy loss scans, which measure the energy loss of the electrons due to the FEL process [5]. We note that even at the nominal heating level, the harmonic content only reached $0.2 \%$, likely due to the poor FEL performance on the day of the measurement. 


\begin{tabular}{|c|c|c|}
\hline Photon Energy & 2nd Harm. & 3rd Harm. \\
\hline Soft X-rays (near 1 keV) & $0.04-0.1 \%$ & $2.0-2.5 \%$ \\
Hard X-rays (6-8 keV) & & $0.2-2 \%$ \\
\hline
\end{tabular}

Table 5.4: Summary of harmonic results. The second harmonic content in normal operation at soft x-ray wavelengths is below $0.1 \%$. The third harmonic content is as high as about $2 \%$ at normal operation, but can drop by an order of magnitude or more due to poor beam quality.

expectations. We confirm that the proportion of third harmonic falls as the FEL performance degrades.

\subsection{Acknowledgements}

We would like to thank S. Biedron and D. Zhu for helpful comments and all members of the LCLS commissioning team for making this work possible. Work is supported by Department of Energy contract DE-AC0276SF00515. 


\section{Chapter 6}

\section{Two-Chicane Compressed Harmonic Generation of Soft X-Rays}

\subsection{Introduction}

In the first chapters of this work, we have treated microbunching phenomena arising from shot noise. We looked at unwanted microbunching due to instabilities (chapter 3) as well as methods for removing shot noise microbunching (chapter 2). We also described measurements on a LCLS, a free electron laser (FEL) driven by the self-amplified spontaneous emission (SASE) process (chapters 4 and 5). A SASE FEL uses an undulator's resonant condition (Eq. 4.3) to select one wavelength from the broad bandwidth shot noise. Though SASE FELs have proved extremely successful [5], they are saddled by long saturation lengths and poor longitudinal coherence. In principle it is possible to drive microbunching from an external radiation source, such as an optical or UV laser. This concept, called 'seeding,' is the subject of the the next two chapters.

FELs 'seeded' by optical or UV lasers promise full coherence and shorter (and cheaper) saturation lengths than from SASE FELs. One leading seeded scheme is high gain harmonic generation (HGHG) [9, 27]. However, single-stage HGHG requires high laser power (expensive and potentially problematic for the FEL process), and is limited to wavelengths around $20 \mathrm{~nm}$ [28]. Multiple-stage HGHG can reach shorter wavelengths, but is more technically challenging. A recent and promising seeding scheme is echo enabled harmonic generation (EEHG), which manipulates hidden structure in phase space to produce high harmonic seeding [29, 30].

Seeding the electron beam prior to bunch compression, which we will call compressed harmonic generation $(\mathrm{CHG})$, is an alternative approach [32, 31, 33, 34]. In this chapter we discuss a variation on $\mathrm{CHG}$ in which dispersion from the compression stage smears out the longitudinal modulation, but the structure remains imprinted in phase space and can be revived later [33, 35].

The scheme uses two dispersive sections, the first to compress the beam and the second to bunch the modulation. Starting from the electron gun, an accelerator section brings the beam to energy $E_{a}$, while adding a linear chirp, $h$. A laser then modulates the beam energy by $A_{L} \cos \left(k_{L} z\right)$. A dispersive section, $R_{56}^{(a)}$, simultaneously compresses the bunch length by a factor of $\alpha=1 /\left(1+R_{56}^{(a)} h\right)$, while also strongly over-bunching the laser modulation. Another accelerator section flattens the beam with a second chirp, $-\alpha h$, while increasing the energy to $E_{b} \equiv g E_{a}$. Finally, a second dispersive section with effective opposite sign, 
$R_{56}^{(b)} \approx-R_{56}^{(a)} g / \alpha \pm g /\left(A_{L} \alpha^{2} k_{L}\right)$, unwinds the over-bunched laser modulation, recovering a maximally bunched beam at wavevector $\alpha k_{L}$. As an added benefit, the second dispersive region also reverses second order effects from the preceding accelerator section. The full multi-step process is summarized in Fig. 6.1.

In this chapter we give an analytical description of the two-chicane CHG scheme, and extend the simulations of [35] to include higher energies and smaller $\beta$-functions (desirable for sending the beam through a radiator). The major drawback of compressed seeding is the need to preserve fine phase space structure across long accelerator regions. In the final section we consider the technical requirements for effective transport of this phase space modulation. This work was published as [75].

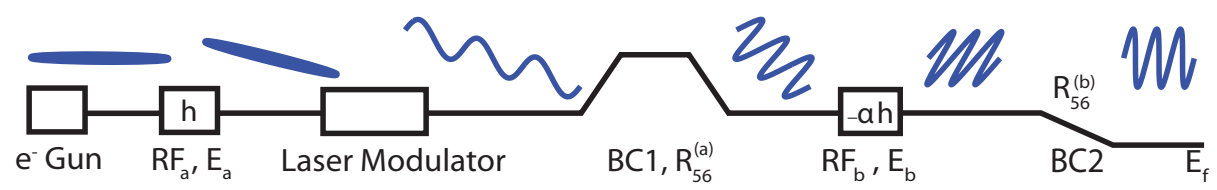

(a)

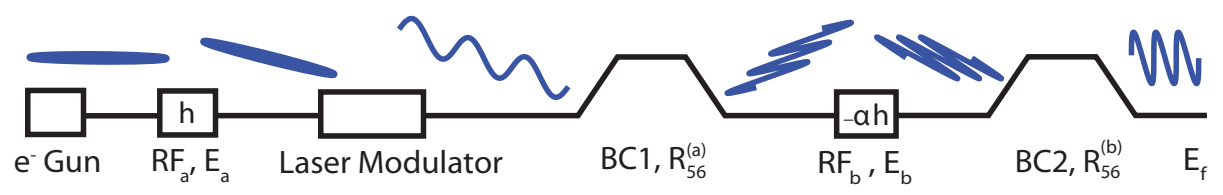

(b)

Figure 6.1: Diagram of CHG scheme. a) The first accelerator section raises the particle energy to $E_{a}$ and introduces a linear chirp, $h$. After modulating with a laser, the first dispersive section, $\mathrm{BC} 1$, compresses the beam and over-bunches the modulation. A second RF section accelerates the particles to $E_{b}$, and cancels the chirp of the first section. The final dispersive section, $\mathrm{BC} 2$, unwinds the over-bunching. b) Operating $\mathrm{BC} 1$ in over-compression rotates the electron beam head-to-tail, allowing the use of a chicane for both $\mathrm{BC} 1$ and BC2. In both cases it is possible to add a third accelerator section to reach a final energy of $E_{f}$.

\subsection{Motivation}

\subsubsection{Advantages of Compressed Seeding}

Seeding before compression offers several advantages. First, the bunch compressor reduces the final modulation wavelength by a factor of $\alpha$. Second, the RF phase controls $\alpha$, so changing the phase tunes the final wavelength. Third, the combination of chirp and bunch compressor amplifies the laser modulation by the compression factor, reducing the required laser power by $\alpha^{2}$. CHG also requires relatively few components, even for the two-chicane variation; the first chicane doubles as the bunch compressor (required to increase current for all FELs), so we need only one additional modulator and chicane, the same as for single-stage HGHG. Finally, the low power requirement allows seeding with short wavelength lasers.

\subsubsection{Advantages of Two Chicane CHG}

The simplest compressed seeding schemes use a single dispersive section to both compress the beam and bunch the laser modulation [32, 31]. For simultaneous compression and bunching, the chirp slope must match the modulation strength; i.e., the same dispersive section, of strength $R_{56}$, both compresses the chirp 
(moving electrons on the order of the mm-scale bunch length) and maximizes the bunching (by compressing the sub- $\mu$ m-scale modulation). This matching condition implies a laser modulation approximately 1000 times weaker than the chirp, limiting the modulation amplitude to a few tens of eV. Such a small amplitude is sufficient for the small energy spread of a thermionic gun (as used in [32]), but is far below the incoherent energy spread in high current, photocathode beams.

To extend CHG to photocathode beams, we have considered the addition of a second dispersive section to allow for larger modulation strengths [35]. The additional dispersive region, $R_{56}^{(b)}$, separates the compression, carried out by the first dispersive section, $R_{56}^{(a)}$, and the bunching condition, determined by the modified sum, $R_{56}^{(T)}=R_{56}^{(a)}+\frac{\alpha}{g} R_{56}^{(b)}$. As a side benefit, the second dispersive region corrects upstream errors, reversing second order effects in the first chicane and subsequent accelerator sections. We note that two-chicane compressed seeding was first suggested in [33]. A modified version of the scheme described in [35] was proposed recently $[76,77]$.

The downside of compressed seeding is the need to preserve the modulation throughout the ensuing accelerator sections. A second dispersive region can help reverse smearing from certain effects (e.g. emittance), but also requires strong dispersion with very tight tolerances. The advantages of $\mathrm{CHG}$ must be weighed against these technical constraints.

\subsection{Analytical Model}

\subsubsection{Bunching Factor}

The seeding can be quantified by the bunching amplitude at the end of $\mathrm{BC} 2$,

$$
b_{f}(k)=\int d z_{f} \int d p_{f} e^{i k z_{f}} \Psi_{f}\left(z_{f}, p_{f}\right)
$$

with final longitudinal particle position, $z_{f}$, normalized energy, $p_{f}$, and final distribution $\Psi_{f}\left(z_{f}, p_{f}\right)$. (For a particle of energy, $E$, we define $p \equiv\left(E-E_{b}\right) / E_{b}$.) To evaluate Eq. 6.1 analytically, we assume an initially uniform longitudinal distribution within the electron beam. We also assume an initial Guassian energy distribution, $\Psi_{i}\left(p_{i}\right) \propto \exp \left[-p_{i}^{2} / 2 \sigma_{p}^{2}\right]$, with relative energy spread, $\sigma_{p} \equiv \sigma_{E 0} / E_{a}$, defined in terms of the absolute energy spread, $\sigma_{E 0}$, and energy prior to the first bunch compressor, $E_{a}$.

We describe the CHG process as follows: 1. We chirp the beam and modulate with a laser. 2 . The first dispersive section (abbreviated BC1) compresses the beam. 3. We accelerate again and add a second chirp. 4. A second dispersive section (BC2) unwinds the over-bunching,

$$
\begin{aligned}
z_{1}=z_{i}, & p_{1}=p_{i}+A_{L} \sin \left(k_{L} z_{i}\right)+h z_{i} \\
z_{2}=z_{1}+R_{56}^{(a)} p_{1}, & p_{2}=p_{1} \\
z_{3}=z_{2}, & p_{3}=\left(p_{2}-\alpha h z_{2}\right) / g \\
z_{f}=z_{3}+R_{56}^{(b)} p_{3}, & p_{f}=p_{3} .
\end{aligned}
$$

If we transform to the initial coordinates, $d z_{f} d p_{f} \rightarrow d z_{i} d p_{i} / g$, we can evaluate Eq. 6.1 by integrating over the simple uncorrelated initial distribution, $\Psi_{i}\left(p_{i}\right)$. Solving for $z_{f}$ in terms of $z_{i}$, we find

$$
z_{f}=z^{\prime}+R_{56}^{(T)}\left[p_{i}+A_{L} \sin \left(k_{L} \alpha z^{\prime}\right)\right]
$$


with definitions $z^{\prime} \equiv z_{i} / \alpha$ and $R_{56}^{(T)} \equiv R_{56}^{(a)}+R_{56}^{(b)} \alpha / g$. We note that Eq. 6.3 has the same form as from a single modulation, but at compressed wavevector $\alpha k_{L}$, and with effective dispersive section, $R_{56}^{(T)}$, and relative laser modulation amplitude, $A_{L} / \sigma_{p}$. It is possible to set $R_{56}^{(T)} \ll R_{56}^{(a)}, R_{56}^{(b)}$, so that a small effective $R_{56}^{(T)}$ bunches the short-wavelength modulation despite the strong dispersion of the bunch compressors. Consequently, the two terms in $R_{56}^{(T)}$ must have opposite signs, requiring either opposite sign dispersive sections (e.g. one chicane, one 2-bend dogleg), or negative $\alpha$ (over-compression) (Fig. 6.1). Chicanes have relatively favorable geometric properties compared to doglegs, so for this chapter we choose negative $\alpha$ and use chicanes for both dispersive regions.

Having changed $d z_{f} d p_{f} \rightarrow d z_{i} d p_{i} / g$, and $\Psi_{f} \rightarrow \Psi_{i}$, we integrate Eq. 6.1 to find bunching at the harmonics as in HGHG [9]

$$
b_{f}\left(m \alpha k_{L}\right) \propto e^{-\frac{\left(m \alpha k_{L} R_{56}^{(T)} \sigma_{p}\right)^{2}}{2}} J_{m}\left(m \alpha k_{L} R_{56}^{(T)} A_{L}\right)
$$

with maxima at $\alpha k_{L} R_{56}^{(T)} A_{L} \approx \pm 1$. To avoid suppression by the energy spread, we require $\left|m \alpha k_{L} R_{56}^{(T)} \sigma_{p}\right|<$ 1 , giving significant bunching at the $m$ th harmonic when $A_{L}>m \sigma_{p}$.

We can now confirm the advantages of CHG. First, we produce radiation at the harmonics of the compressed wavelength $\lambda_{L} / \alpha \ll \lambda_{L}$. Second, the RF phase can shift $|\alpha|$, tuning the final wavelength. Third, the pre-compression energy spread, $\sigma_{p}$, rather than the larger post-compression energy spread, $|\alpha| \sigma_{p}$, determines the required laser modulation amplitude, $A_{L}$. A 1D simulation with parameters from Table 7.1 illustrates the process (Fig. 6.2).

\subsubsection{Error Self-Correction from Second Dispersive Region}

The primary appeal of employing two dispersive regions is the separation of the bunching and compression requirements. However, the second dispersive region provides a second advantage by reversing longitudinal smearing. In our simple model (Eq. 6.2), we assumed a longitudinally frozen beam outside of the dispersion regions $\mathrm{BC} 1$ and $\mathrm{BC} 2$. In reality, longitudinal deviations, such as second order transport elements, will smear out the phase space structure even outside of dispersive regions. The second chirp and dispersive region help to cancel such effects.

We model the self-correction in 3 steps. 1. The $j$ th particle acquires a longitudinal error, $\Delta z_{j}$, due to second order effects following $\mathrm{BC} 1$. 2. The second chirp imparts a relative energy modulation of $\Delta E_{j}=$ $-\frac{\alpha}{g} h \Delta z_{j} .3$. BC2 produces a longitudinal shift, resulting in the final longitudinal error

$$
\Delta z_{j}^{(\mathrm{F})}=\Delta z_{j}-R_{56}^{(b)} \frac{\alpha}{g} h \Delta z_{j} \approx \Delta z_{j} / \alpha
$$

where we have assumed $R_{56}^{(a)}, R_{56}^{(b)} \gg R_{56}^{(T)}$ so that we can approximate $R_{56}^{(b)} \approx-g R_{56}^{(a)} / \alpha$ in the second step. We find that the second chirp and dispersive regions effectively re-compress the phase space structure, reducing smearing between the two chirps by the compression factor, $|\alpha|$. (Smearing from within the chirps and second chicane is suppressed more weakly, due to the decreased effective values of $h$ and $R_{56}^{(b)}$.) This recompression of the fine density modulation is essential to $\mathrm{CHG}$ at short wavelengths. We consider a specific case of error self-correction in Section 6.5.2. 

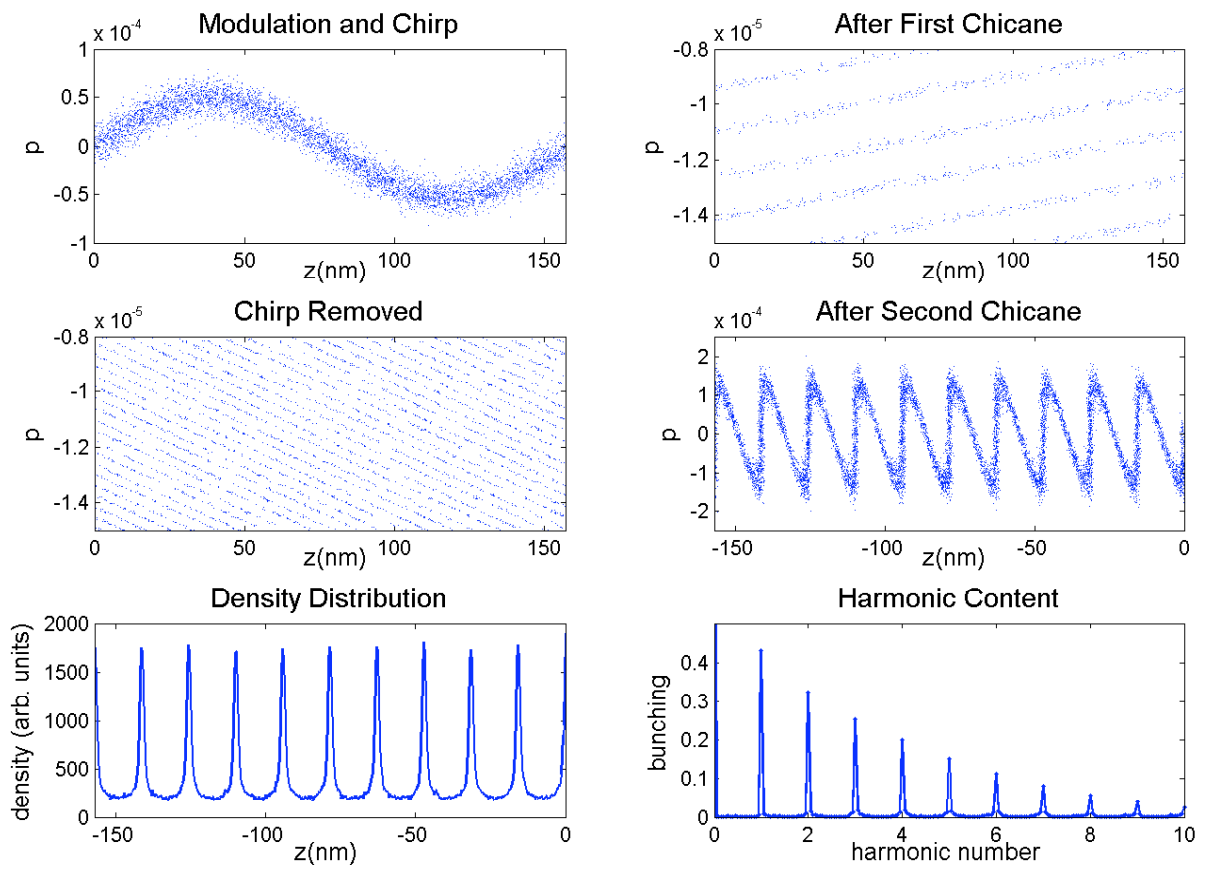

Figure 6.2: In the first step (upper left) we add a chirp and laser modulation. We show only one modulation wavelength, so the chirp effect is small. After the first chicane, the modulation is over-bunched (zoom, upper right). A second chirp reverses the first chirp (zoom, middle left, shows reversed slope in phase space). After more acceleration, the second chicane revives bunching (middle right). Note the compression of the wavelength by $|\alpha|=10$, and the increase in modulation amplitude by $|\alpha| / g=2.5$. We find strong bunching past the 5th harmonic (bottom).

\subsection{Simulations}

\subsubsection{Simulation Inputs}

To test the practicality of a CHG scheme, we have constructed a sample accelerator lattice (Fig. 6.3) using MAD [78]. We emphasize that we have not fully optimized the lattice, but use it merely as a demonstration to study tolerances. We then simulate the CHG process by particle tracking in elegant [6]. The simulations include incoherent synchrotron radiation (ISR) and first and second order transport elements for both longitudinal and transverse motion. We have not simulated coherent effects (e.g. coherent synchrotron radiation in the chicanes) due to computational constraints. To simulate the modulation, we use a $6 \mathrm{MW}$ laser with $2 \mathrm{~mm}$ focal width, interacting with the beam over a 12 period, $50 \mathrm{~cm}$ long undulator. The undulator has sufficient bandwidth to seed the entire beam even with a strong $2 \%$ chirp. We assume a $157 \mathrm{~nm}$ modulation wavelength (corresponding to an $\mathrm{F}_{2}$ laser), so that with compression of $|\alpha| \approx 10$, we find $3 \mathrm{~nm}$ radiation at the 5th harmonic of the density modulation (50th harmonic of the initial $\lambda_{L}$ ). By modulating at low energy $(250 \mathrm{MeV})$, the moderate laser power still produces a relative modulation of $A_{L}=5 \times 10^{-5}$. A partial list of parameters used in the simulations can be found in Table 7.1 and simulation results are given in Figs. 6.4 
and 6.5 .

\begin{tabular}{|l|c|}
\hline Laser wavelength $\left(\lambda_{L}\right)$ & $157 \mathrm{~nm}$ \\
Laser power $\left(P_{L}\right)$ & $6 \mathrm{MW}$ \\
Laser modulation amplitude $\left(A_{L}\right)$ & $5 \times 10^{-5}$ \\
Uncorr. RMS E-spread before BC1 $\left(\sigma_{p}\right)$ & $1 \times 10^{-5}$ \\
Norm. transverse emittance $\left(\epsilon_{N}\right)$ & $0.4 \mu \mathrm{m}$ \\
Initial energy spread $\left(\sigma_{E 0}\right)$ & $2.5 \mathrm{keV}$ \\
Initial bunch length $\left(\sigma_{z}\right)$ & $400 \mu \mathrm{m}$ \\
Electron energy $\left(E_{a}, E_{b}, E_{f}\right)$ & $0.25,1.0,3.5 \mathrm{GeV}$ \\
BC1, BC2 dispersion $\left(R_{56}^{(a)}, R_{56}^{(b)}\right)$ & $50 \mathrm{~mm}, 20 \mathrm{~mm}$ \\
Compression factor $(|\alpha|)$ & 10 \\
Linac RF Frequency & $1.3,3.9 \mathrm{GHz}$ \\
\hline
\end{tabular}

Table 6.1: Parameter list for both 1D (MATLAB) and 3D (elegant) simulations. We choose L-band RF frequencies because the larger diameter structures will mitigate the effect of wakefield fluctuations. We have not listed bunch charge because without wakefield effects the simulations are charge-independent.

We divide both the first and second linac sections into two parts, with the first half providing acceleration (phase 'on-crest') and the second providing chirp (phase at 'zero-crossing'). We assume accelerating gradients of approximately $25 \mathrm{MV} / \mathrm{m}$ for each linac section. A third harmonic cavity linearizes the phase space prior to the first bunch compressor, providing a flat central region (left plot, Fig. 6.4). We do not require a third harmonic cavity in the second stage, because after compression, the bunch length $\left(\sigma_{z} /|\alpha| \sim 40 \mu \mathrm{m}\right)$ is short compared to the RF wavelength $\left(\lambda_{r f} \sim 20 \mathrm{~cm}\right)$. We then find that the deviation due to RF curvature, $1-\cos \left(k_{r f} \sigma_{z} / \alpha\right)$, is negligible compared to the final energy spread $\left(|\alpha| A_{L} \sim 5 \times 10^{-4}\right)$.

The compression and bunching of the modulation is evident in a zoom of the phase space (right plot, Fig. 6.4). 'Standing up' the modulation produces sharp density spikes with high harmonic bunching. To calculate the bunching factor for bandwidth $\Delta \lambda / \lambda$ at a position $z_{0}$, we sum the particle phases, $b(k)=$ $\sum_{j} \exp i k z_{j}$, for all particles in the region $z_{0}-\lambda / 2 \Delta \lambda<z_{j}<z_{0}+\lambda / 2 \Delta \lambda$. For bunching at the fifth harmonic of the compressed modulation, we set $k=5|\alpha| k_{L}$. The result is given in Fig. 6.5.

\subsubsection{Radiation}

With high levels of density modulation from the seeding process, we can pass the electron bunch directly into a radiator. Assuming a $12 \%$ bunching factor and 30 meter $\beta$-function at the end of the lattice, feeding a 3.5 $\mathrm{GeV}, 1 \mathrm{kA}$ beam through a 2.5 meter undulator with $K=3.5$ and $4 \mathrm{~cm}$ period produces nearly $10 \mathrm{MW}$ of power. The beam could also feed a radiator directly following the second chicane, where the lower energy and larger $\beta$-function are partially compensated by the higher bunching factor (above $15 \%$ ) and shorter accelerator.

Alternatively, the final pre-modulated 3.5 GeV beam could drive an FEL. For the parameters of Table 7.1 with kA beam current we find a Pierce parameter of $\rho \approx 10^{-3}$ [11]. The strong initial bunching requires only a few gain lengths of undulators to reach saturation, where Ming Xie scaling estimates nearly 5 GW of power [79]. 


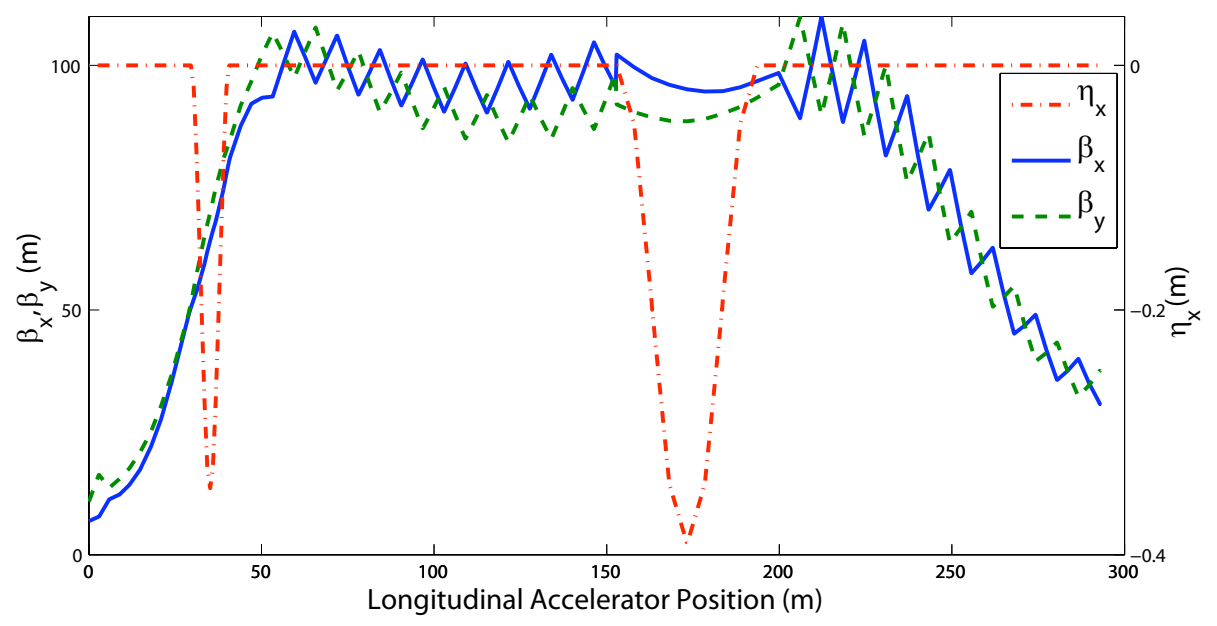

Figure 6.3: Courant-Snyder parameters for 3D elegant simulations showing $\beta$-functions (solid and dotted lines) and dispersion ( $\eta_{x}$, dashed-dotted line). The weak second chicane is designed to minimize ISR effects. Following the second chicane, a third linac section increases the electron energy while simultaneously decreasing the $\beta$ function, enhancing the radiation power.

\subsection{Feasibility}

The drawback to compressed seeding is the need to preserve the phase space modulation across long accelerator regions. In particular, CHG is most sensitive to errors originating between the dispersive sections; errors prior to the first chicane are largely canceled by the second chicane. In the following section we study tolerances by estimating the effects of incoherent synchrotron radiation (ISR), dispersion errors, longitudinal smearing from 3D effects, and changes to the bunching condition due to jitter in the second chirp strength. Finally, we consider how modulating near the beginning of the accelerator may affect the microbunching instability.

\subsubsection{ISR Induced Energy Spread}

Incoherent energy spread, $\Delta p^{(\mathrm{ISR})}$, from ISR between the two dispersive sections could destroy the fine phase space structure. To preserve high harmonics, we must limit the longitudinal broadening, $\Delta z^{\left(\sigma_{p}\right)}$, from the total energy spread to less than $\lambda_{L} / 2 \pi m|\alpha| \sim 0.5 \mathrm{~nm}$. We can estimate

$$
\Delta z^{\left(\sigma_{p}\right)}=R_{56}^{(a)} \sigma_{p}+R_{56}^{(b)} \frac{\alpha}{g} \sigma_{p}+R_{56}^{(b)} \Delta p^{(\mathrm{ISR})}
$$

where $\sigma_{p}$ is the uncorrelated relative energy spread at $\mathrm{BC} 1$. The sum of the first two terms is small by design, so for $R_{56}^{(b)}=20 \mathrm{~mm}$, we find $\Delta p^{(\mathrm{ISR})} \sim 10^{-8}$ for each bend, achievable with weak dipoles in BC2 at $1 \mathrm{GeV}$ (Fig. 6.3). Fig. 6.5 shows bunching including ISR effects.

\subsubsection{Second Order Lattice Effects}

The laser modulation must survive transport through two strong chicanes, and approximately $60 \mathrm{~m}$ of accelerator. Smearing from second order effects (e.g. emittance and curvature from energy modulation) could 


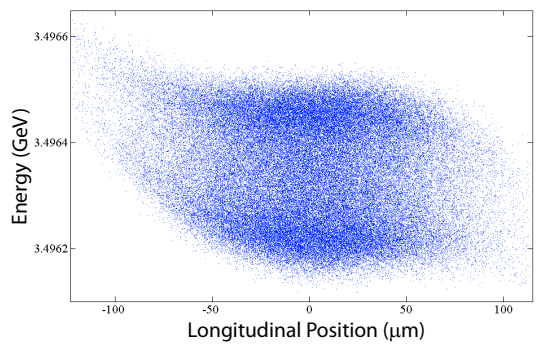

(a)

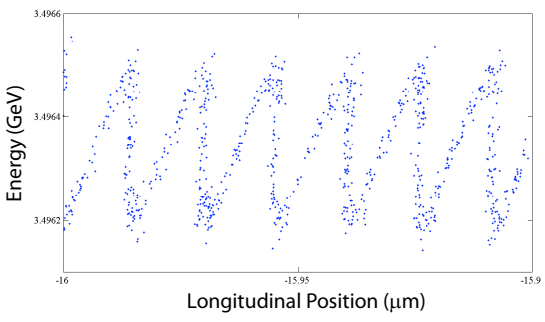

(b)

Figure 6.4: a) Particle phase space for elegant simulation of parameters in Table 7.1. The two horizontal stripes of higher density are signatures of a sinusoidal modulation. A third-harmonic accelerating cavity produces the flat central portion of the beam. b) A zoom of the phase space shows the compressed modulation bunched to optimize higher harmonics. The vertical stripes ('standing up' the modulation) in phase space produce sharp density spikes that drive harmonic generation. The $T_{566}$ component of the transfer matrix causes slight scalloping.

broaden the fine $3 \mathrm{~nm}$ structure. An electron with coordinates $X=\left[x, x^{\prime}, y, y^{\prime}, z, p\right]$ acquires a longitudinal deviation of $\Delta z=\tilde{X} T_{5 i j} X$, with $T_{5 i j}$ the second order transport coefficient from the modulator to the beginning of the second chirp. As described in Section 6.3.2, the second chirp helps to cancel such effects. The chirp imparts a relative energy modulation of $-\frac{\alpha}{g} h \Delta z$, reducing the longitudinal shift following the second chicane by a factor of $|\alpha|$. Though the re-compression is weaker for errors from further down the accelerator, for our lattice (Fig. 6.3), the transverse components $\left(T_{5 i j}\right)$ are dominated by the first chicane, where we increase the beta function after the small beam radius of the modulator. As a result, we expect the error self-correction effect to be significant for our sample lattice.

To estimate the size of the second order terms, we tracked a longitudinal delta slice (vanishing bunch length and energy spread, but finite emittance) from the modulation to the end of the accelerator. We observe that the acceleration following the first chicane broadens the slice, but also imparts a chirp. The second chicane then re-compresses the slice, decreasing the smearing effect by approximately the compression factor, $|\alpha|$ (Fig. 6.6).

\subsubsection{RF Phase and Wakefield Stability}

RF phase errors, wakefields, and RF curvature alter the linear chirp between dispersive sections and degrade the final bunching. An error in the second, canceling chirp shifts the unwinding process and leaves the modulation either under or over-bunched. (The first chirp is less problematic because the two dispersive sections have canceling effects.)

To estimate the sensitivity to a linear chirp error, we repeat the earlier analysis, with the addition of an error, $\varepsilon$, in the final chirp. Step three becomes

$$
z_{3}=z_{2}, \quad p_{3}=\left(p_{2}-\alpha h z_{2}-\varepsilon z_{2}\right) / g .
$$

Again solving for $z_{f}$ in terms of $z_{i}$, we find

$$
z_{f}=z^{\prime}+\left(R_{56}^{(T)}-\delta_{R}\right)\left[p_{i}+A_{L} \sin \left(\frac{\alpha k_{L}}{1-\delta_{k}} z^{\prime}\right)\right]
$$




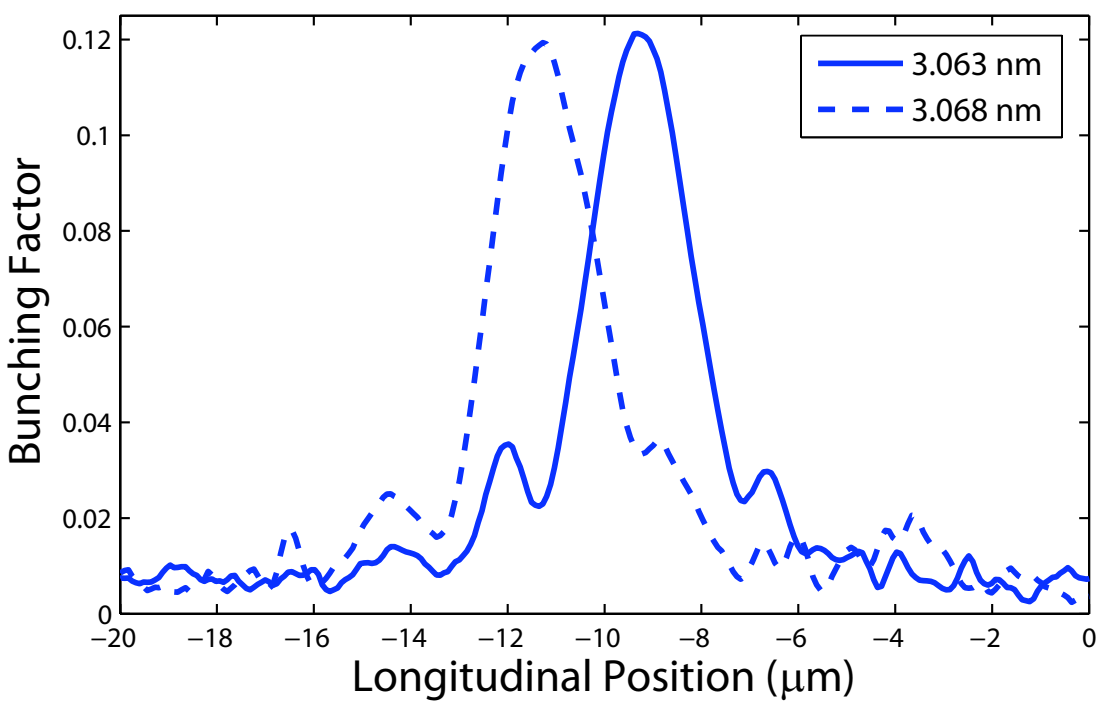

Figure 6.5: Bunching factor at two wavelengths with $0.15 \%$ bandwidth from elegant [6] simulation (parameters in Table 7.1). Bunching is lower than for the 1D case because of 2nd order effects (e.g. emittance and $T_{566}$ ), ISR and finite laser radius.

with definitions $\delta_{k} \equiv R_{56}^{(b)} \varepsilon / g, \delta_{R} \equiv R_{56}^{(a)} R_{56}^{(b)} \varepsilon / g$, and $z^{\prime} \equiv z_{i}\left(1-\delta_{k}\right) / \alpha$. The chirp error has two effects: the wavevector shifts by the factor $1-\delta_{k}$ and the $R_{56}^{(T)}$ required to unwind the over-compression shifts by $\delta_{R}$. While the shift in wavevector is small, the second condition implies a tight constraint on the phase stability of the second chirp; if the phase shifts, the effective $R_{56}^{(T)}$ either over or under-bunches the modulation. To maintain maximal bunching we need chirp error $|\varepsilon| \ll g R_{56}^{(T)} / R_{56}^{(a)} R_{56}^{(b)} \approx 0.2 / \mathrm{m}$, requiring control of the second chirp to better than $0.1 \%$. The dot-dash curve in Fig. 6.7 shows bunching decreases to $10 \%$ with a \pm 0.005 degrees phase error.

Longitudinal space charge wakefields produce an energy modulation that is proportional to the derivative of the beam density profile. For a Gaussian bunch, we approximate the wakefield effect as an additional linear chirp near the center of the beam. (There will be nonlinear effects at the edges, but we are primarily interested in the central region where CHG is most effective). With charge fluctuations of $\sim 1 \%$, we can only ignore the wakefield if it contributes less than $10 \%$ of the chirp. To minimize the wakes, we assume L-band superconducting structures with large geometric apertures.

\subsubsection{Dispersion Leakage}

The most serious technical limitations come from dispersion leakage in the first chicane, which can smear the fine structure over the ensuing accelerator sections. For example, jitter in the dipole magnet strengths and quadrupole terms in the magnets will both broaden the modulation. We study dispersion leakage by tracking a longitudinal delta slice of finite emittance, but vanishing bunch length and energy spread (as we did for second order effects). We then adjust the field strength and quadrupole terms of a bend magnet in the first chicane, and observe the effect on the final bunch length. For the bend magnets in the middle of the chicane, we find the quadrupole jitter must have geometric strengths below $K=10^{-5}$ (corresponding to fields of 0.1 

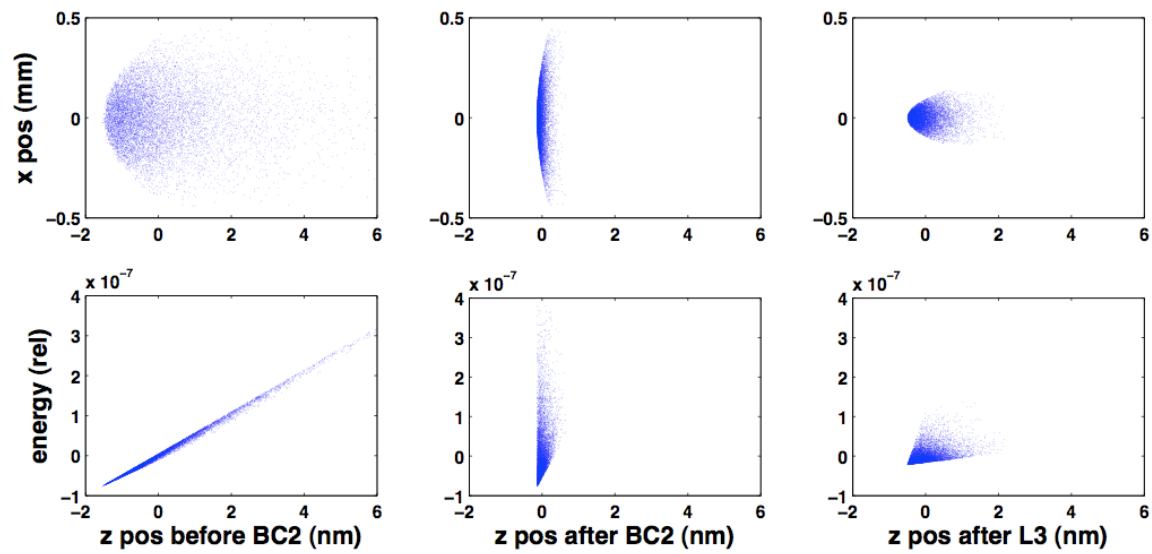

Figure 6.6: To demonstrate the emittance cancelation effect, we track a longitudinal delta slice (zero length) following the laser modulation. Emittance effects increase the slice length before the second chicane, BC2, (top left, $x$ vs. $z$ ), but the off-crest accelerating section introduces a chirp to the beam (bottom left, energy vs. $z$ ). The second chicane then re-compresses the bunch by the compression factor, $|\alpha|$ (center). Following the final accelerator section, L3, the slice starts to spread out again (right). Bunch head is to the left.

$\mathrm{G} / \mathrm{m}$ ) to preserve $3 \mathrm{~nm}$ bunching. Likewise, jitter in the dipole strengths must be kept below $0.01 \%$. Both effects are primarily second order, so they decrease with shorter accelerating sections (i.e. higher accelerating gradients), which can be achieved with higher frequency accelerating structures. We note that the laser chirp method of Ref [76] may be less sensitive to dispersion errors due to the short distance between chicanes. However, higher frequency structures and laser chirps may both be highly susceptible to phase errors, as discussed in the previous section. Lowering the $\mathrm{x}$-emittance (e.g. in $20 \mathrm{pC}$ operation [80]) may also improve the tolerances.

In a CHG scheme, the tolerances for the accelerator are comparable to those of an undulator in an FEL; in both cases, preservation of the fine bunching is essential. For this reason, we also track a longitudinal delta slice while shifting the position of individual dipoles and quadrupoles. We find longitudinal errors of below $1 \mathrm{~mm}$ in the bend magnets and individual quadrupole offsets below $300 \mu \mathrm{m}$ have negligible smearing effect. Finally, we note that all tolerances are looser if we place the radiator directly following BC2.

\subsubsection{Microbunching}

The microbunching instability (MBI) can degrade linac and FEL performance [44]. Incoherent energy spread damps the MBI effect by the exponential factor $e^{-k^{2} R_{56}^{2} \sigma_{p}^{2}}$, suppressing radiation for $k R_{56} \sigma_{p}>1$. For the parameters of Table 7.1, we expect suppression of MBI effects for $\lambda<3 \mu \mathrm{m}$. If long wavelength MBI is still problematic, a laser heater could push the incoherent energy spread as high as $10 \mathrm{keV}$, damping radiation out to wavelengths of $12 \mu \mathrm{m}[21,22]$.

While laser heaters have proved effective at suppressing MBI [4], the larger initial energy spread would require higher seed laser power and enhance distortions from $T_{566}$. Given the similarity between the laser heater and the first stage of $\mathrm{CHG}$, it is interesting to consider the extent to which the $\mathrm{CHG}$ process naturally suppresses MBI without additional heating. 


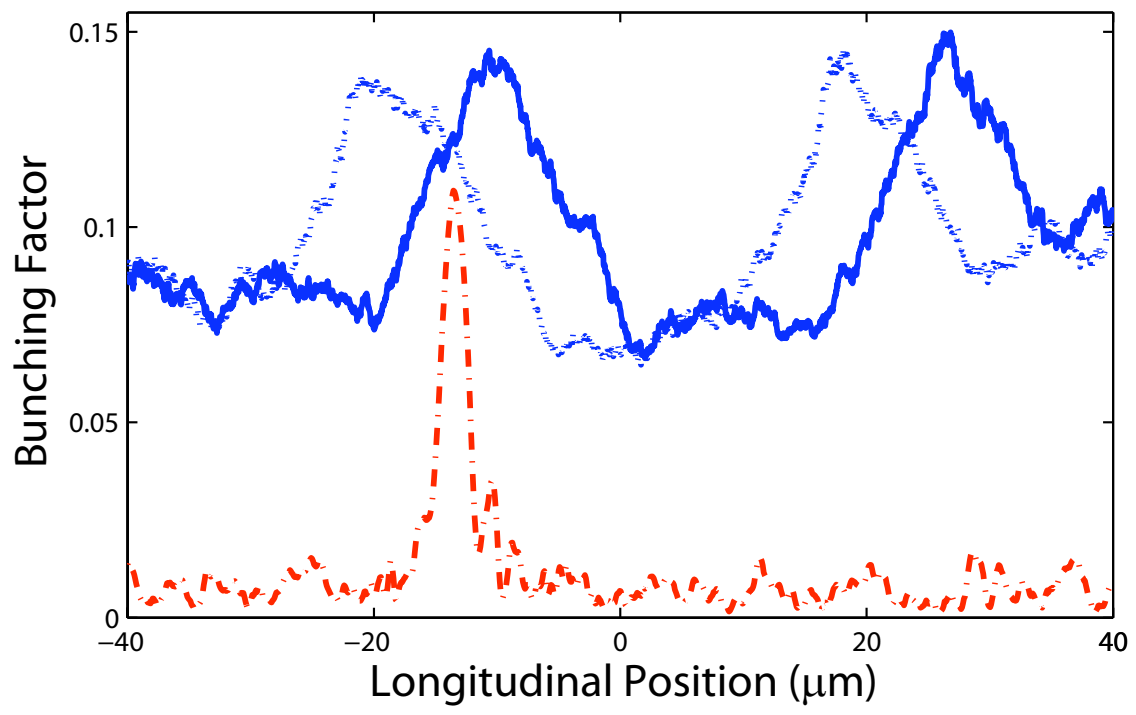

Figure 6.7: Wide bandwidth bunching for two simulations with a 0.01 degree phase shift (solid and dotted blue lines). An FEL would pick out a narrow $\Delta \lambda / \lambda=0.15 \%$ bandwidth, leaving lower bunching (narrow peak, red dot-dash line).To calculate the bunching factor at a position $z_{0}$ and bandwidth $\Delta \lambda / \lambda$, we sum the phases of all particles in the region $z_{0}-\lambda / 2 \Delta \lambda<z<z_{0}+\lambda / 2 \Delta \lambda$. The high baseline in the wide bandwidth curves is due to the relatively low number of particles per bunching calculation when $\lambda / \Delta \lambda$ is small. A bunched sine wave makes a sawtooth that may either be right-leaning, as in Fig. 6.4, or left-leaning, depending on the sign of the effective dispersion, $R_{56}^{(T)}$. In our simulation, $R_{56}^{(T)}$ changes as a function of longitudinal beam position, resulting in the double peak seen in the wide bandwidth bunching.

We model the MBI in two steps; first, shot noise in the electron beam produces broadband space charge forces that modulate the beam energy. Second, a dispersive region converts the energy modulation into amplified density modulation. To evaluate the MBI amplitude, we rewrite the first line of Eq. 6.4 with the addition of a shot noise driven energy term, $A_{s n} \sin \left(k_{s n} z_{i}\right)$, with the understanding that the wavevector $k_{s n}$ may take on any value, and the amplitude, $A_{s n}$, is generally small. As in Section 6.3.1, our strategy is to use the normalized initial coordinates, $z_{i}^{\prime} \equiv z_{i} / \alpha$, to express the coordinates following the first chicane,

$$
z_{f}=z_{i}^{\prime}+R_{56} p_{i}+R_{56}\left[A_{L} \sin \left(\alpha k_{L} z_{i}^{\prime}\right)+A_{s n} \sin \left(\alpha k_{s n} z_{i}^{\prime}\right)\right] .
$$

If we again assume a uniform longitudinal distribution with Gaussian energy spread, $\Psi_{i}\left(p_{i}\right)$, we find the bunching factor (from Eq. 6.1) due to MBI

$$
\langle b(k)\rangle \propto e^{-\frac{k^{2} R_{56}^{2} \sigma_{p}^{2}}{2}} \alpha k_{s n} R_{56} A_{s n} \sum_{n} J_{n}\left(k R_{56} A_{L}\right) \int d z_{i}^{\prime} e^{-i k z_{i}^{\prime}} e^{i n \alpha k_{L} z_{i}^{\prime}}\left(\frac{e^{i \alpha k_{s n} z_{i}^{\prime}}-e^{-i \alpha k_{s n} z_{i}^{\prime}}}{2}\right)
$$

where we have used the Jacobi-Anger expansion on the laser modulation term, and we have assumed we are operating in the regime $k_{s n} R_{56} A_{s n} \ll 1$ to linearize the shot noise term. We expect significant bunching at frequencies that satisfy $k=n \alpha k_{L} \pm \alpha k_{s n}$. The initial, uncorrelated, Gaussian energy spread again drives the exponential pre-factor, while the smeared laser modulation provides additional suppression by way of the Bessel function factor. 
If we consider only long wavelength $\operatorname{MBI}\left(n=0\right.$, so that $\left.k=k_{s n}\right)$, we reproduce Eq. 11 from Ref. [22]; the laser modulation suppresses the MBI by an additional factor of $J_{0}\left(k R_{56} A_{L}\right)$, so we now expect moderate damping for $k R_{56} A_{L} \gtrsim 1.5$. Compared to the exponential pre-factor, the laser suppression extends to longer wavelengths by the ratio $A_{L} / \sigma_{p}$, though the slower decay of the Bessel function weakens the effect. For the parameters of Table 7.1, we find additional suppression at wavelengths as long as $10 \mu \mathrm{m}$ even without the use of a laser heater.

When $n \neq 0$, there may be additional long wavelength MBI driven by frequency beating between the shot noise and seed laser. For example, with $n=-1$, we have long wavelength $k=-k_{L}+k_{s n}$ for $k_{L} \sim k_{s n} \gg k$. Further studies are needed to fully characterize the microbunching instability in CHG.

It is worth noting that the weak Bessel suppression stems from the double-horn energy distribution of a sinusoidal modulation [22]. A perfectly flat energy distribution (a longitudinal sawtooth) would maximize both the microbunching suppression and high harmonic bunching. Even a two-frequency laser modulation approximating a sawtooth would substantially improve the suppression over the single frequency case analyzed above (Fig. 6.8).

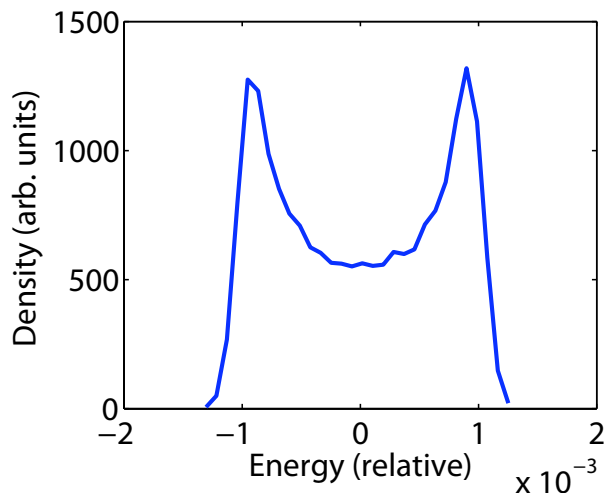

(a)

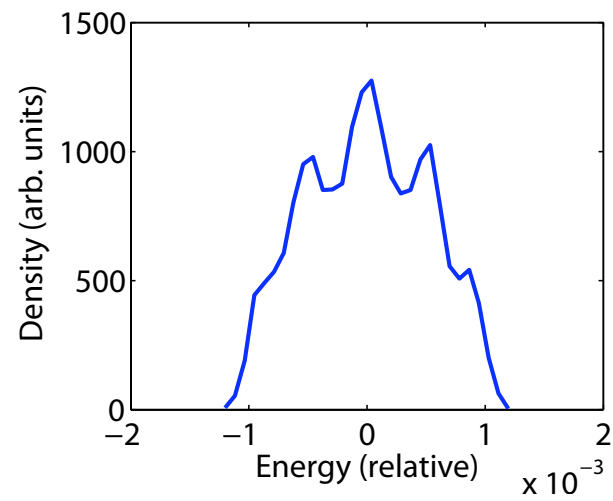

(b)

Figure 6.8: Double-horn energy distribution, left, from a sinusoidal modulation. Approximating a sawtooth with two frequencies gives the more uniform energy distribution at right.

\subsection{Conclusion}

Seeding an electron beam prior to the compression stage provides short wavelength bunching with relatively low seed laser power. In this chapter, we have studied a scheme for two-stage CHG, which extends the method to photocathode beams, and aids in the transport of the fine phase space structure. Through 3D simulations, we have demonstrated that modulation of a beam with $0.4 \mu$ m emittance can survive an extended accelerator

region and still provide bunching at higher harmonics. We have also considered an effect by which smearing errors may be suppressed. However, strict stability requirements on the RF phase (to suppress variation in the chirp) and tight limits on dispersion leakage from the chicanes (to prevent smearing of the fine phase space structure) may make practical implementation of $\mathrm{CHG}$ schemes challenging. 


\subsection{Acknowledgments}

We would like to thank Y. Ding, P. Emma, H. Geng, H. Merdji, G. Stupakov, and D. Xiang for helpful discussions. Work is supported by Department of Energy contract DE-AC02-76SF00515. 


\section{Chapter 7}

\section{Steady-State Microbunching in a Storage Ring for Generating Coherent Radiation}

\subsection{Introduction}

In a coherent electron-based radiation source, electrons group into microbunches spaced at the wavelength of the output radiation. With all electrons radiating at the same phase, the electromagnetic field amplitudes add in phase and the radiated power scales as the square of the number of electrons. The SASE FEL process, for example, amplifies a narrow bandwidth of noise from a random stream of electrons to create a periodic microbunching, which then radiates coherently. Alternatively, we may drive the microbunching with an external radiation source, as described in Chapter 6. In both cases the resulting coherent light can be orders of magnitude brighter than that of an equivalent incoherent source.

For high average power light sources, duty-cycle shares the stage with coherence. Linac driven FELs use each electron pulse once, leading to low duty cycles. (Energy recovery linacs reach high duty cycles by recovering the electron energy $[36,37,38]$.) Storage rings, by contrast, naturally operate at $\mathrm{MHz}$ repetition rates (determined by the revolution time of the electrons), and fully filled rings can provide CW radiation. However, storage rings do not generally support sustained microbunching (MB).

Using an optical or RF modulation, we propose to microbunch stored electrons during each pass through a radiator. Though the electrons may appear smeared elsewhere in the ring, the MB is permanent at the radiator, so we consider this steady-state microbunching (SSMB). The result is a coherent radiation source with $\mathrm{MHz}$ to $\mathrm{CW}$ repetition rate.

In a conventional storage ring, RF 'buckets' both accelerate and trap electrons. The RF modulation accelerates electrons in front of the stable point more than those behind the stable point, so that all particles tend to move toward the stable point due to the ring's dispersion. Instead of a continuous stream of electrons, we find a train of tightly 'bunched' beamlets spaced at the RF wavelength. Replacing the RF with an optical laser results in a beam bunched at optical wavelengths [81].

The drawback to bucket bunching is that the output radiation is limited to the initial radiation wavelength; 
to produce high power radiation at a wavelength, $\lambda_{\text {out }}$, we need bunching at $\lambda_{\text {out }}$, which in turn requires high power radiation at $\lambda_{\text {out }}$ to produce the bunching. Instead, we propose to modulate with an easily available initial wavelength $\lambda_{\text {in }}$ (e.g. optical), but generate stable points distributed at either a harmonic or a multiple of $\lambda_{\text {in }}$. To distinguish harmonic or multiplied bunching from conventional RF buckets, we will refer to trapping at $\lambda_{\text {out }} \neq \lambda_{\text {in }}$ as MB. For example, seeding with $\lambda_{\text {in }}$ between $200 \mathrm{~nm}$ and $2 \mu \mathrm{m}$ (easily available from commercial sources), we can produce MB at wavelengths ranging from $\lambda_{\text {out }}=13.5 \mathrm{~nm}$ to $1 \mathrm{~mm}$. We note that single-shot versions of MB $\left(\lambda_{\text {in }} \neq \lambda_{\text {out }}\right)$ are mainstays of FEL seeding (see e.g.[9, 29]). Portions of this chapter were published as [82].

\subsection{SSMB Mechanism}

\subsubsection{Zero-Crossing SSMB}

Our goal is to establish stable fixed points in phase space that will microbunch the electron beam. A particle is at a fixed point in phase space if, after $T$ turns around the ring, the particle returns to its initial coordinates; with a one turn map $M$, a fixed point exists in phase space at $X_{0}=(z, p)$ if $M^{T} X_{0}=X_{0}$. Each RF bucket contains only one fixed point per wavelength (at $z=m \lambda_{\mathrm{in}}, p=0$, for integers $m$ ), so the standard RF bucket generates bunching, not MB.

(In all discussions, energy, $p \equiv\left(E-E_{\text {beam }}\right) / E_{\text {beam }}$, and position, $z$, are given relative to the ideal electron defined as $p_{I} \equiv 0, z_{I} \equiv 0$. We will consider the case of operation above transition. Below transition, the slippage reverses.)

As an example of $\mathrm{MB}$, we return to the particle at a zero-crossing of a sine modulation. Instead of $p=0$, we now consider a particle with a special energy $p=\Delta p$, such that the particle slips backward by $\Delta z=\lambda_{\text {in }}$ each turn due to dispersion. The particle does not return to its initial position, but because of the modulation's periodicity, the particle moves to an equivalent zero-crossing, so we still consider this to be a fixed point. At each zero-crossing, we find a set of such fixed points arrayed at energies $p=n \Delta p$, for $n=0, \pm 1, \pm 2$, etc. Fixed points with positive (negative) energies slip backward (forward) $n$ wavelengths per turn.

After a full turn, at the ring's modulation point, the microbunches stack at $z=m \lambda_{\text {in }}$, with one stack per modulation wavelength, i.e., the electrons are bunched at $\lambda_{\text {in }}$. However, after a fraction, $1 / H$, of a turn, the fixed points have slipped only $\Delta z=n \lambda_{\text {in }} / H$, and the particles are spaced at the $H^{\text {th }}$ harmonic of $\lambda_{\text {in }}$, i.e. are microbunched at $\lambda_{\text {out }}=\lambda_{\text {in }} / H$ (Fig. 7.1).

\subsubsection{Double Modulations}

For a general account of the SSMB principle we describe a two-stage system; the second modulation and dispersive region improves control of phase space at a small cost in complexity (Fig. 7.2). As in Chapter 6, we break each turn into 4 steps:

$$
\begin{array}{rlrl}
z_{1}=z_{0}, & & p_{1}=p_{0}+F_{a}\left(z_{0}\right) \\
z_{2}=z_{1}+R_{56}^{(a)} p_{1}, & p_{2}=p_{1} \\
z_{3}=z_{2}, & p_{3}=p_{2}+F_{b}\left(z_{2}\right) \\
z_{4}=z_{3}+R_{56}^{(b)} p_{3}, & p_{4}=p_{3} .
\end{array}
$$



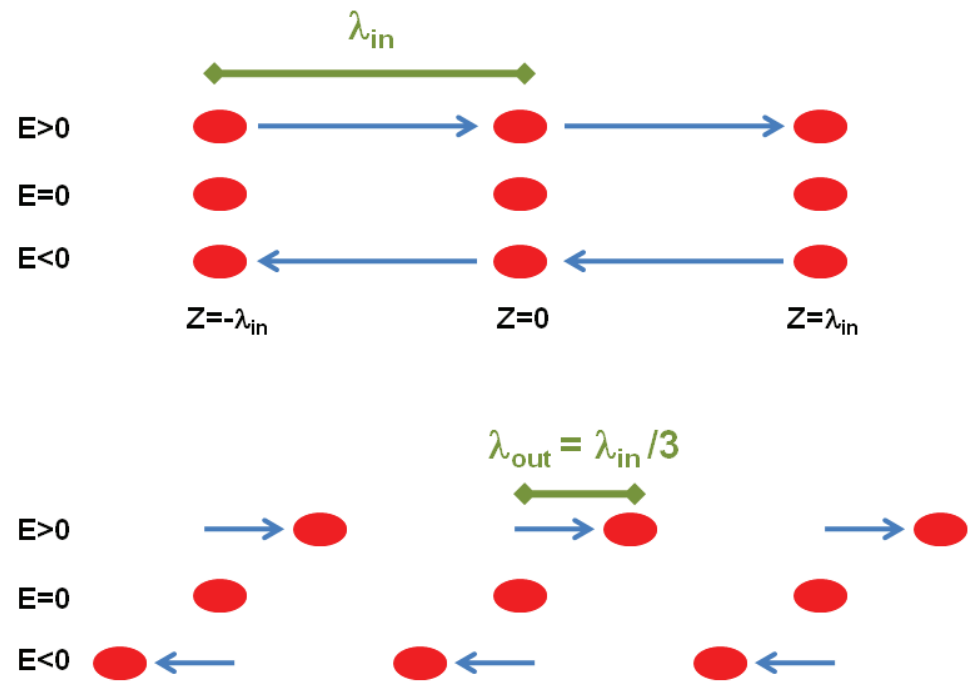

Figure 7.1: An illustration of harmonic SSMB for $H=3$. At top, we show particles in phase space at the modulator. Each turn around the ring, particles slip forward or backward from dispersion, but the distribution is stationary for a periodic modulation. At bottom, at an intermediate point in the ring ( $1 / H$ the way around), the microbunches are spaced by $\lambda_{\text {in }} / H$, i.e. the beam is microbunched with $\lambda_{\text {out }}=\lambda_{\text {in }} / H$.

In step 1 , we apply a modulation to the beam energy, $F_{a}\left(z_{0}\right)$. In step 2 , dispersion $R_{56}^{(a)}$ converts the change in energy to a change in position. We then repeat with a second modulation, $F_{b}\left(z_{2}\right)$, and dispersive section, $R_{56}^{(b)}$. A single pass corresponds to the case of Chapter 6 (but without the chirp). For SSMB, we assume the four steps repeat for each pass around the ring until the beam reaches its steady-state configuration. Taking the case of $T=1$, we then find fixed points whenever we satisfy the slippage condition:

$$
\begin{aligned}
& \Delta z_{\text {onepass }}=R_{56}^{(a)} p_{1}+R_{56}^{(b)} p_{3}=n \lambda_{\text {in }} \\
& \Delta p_{\text {onepass }}=F_{a}\left(z_{0}\right)+F_{b}\left(z_{2}\right)=0,
\end{aligned}
$$

for $n=0, \pm 1, \pm 2$, etc.

Most generally, we are looking for any combination of $R_{56}^{(a)}, R_{56}^{(b)}, F_{a}, F_{b}$ such that the resulting fixed points have a clean, periodic structure. In our previous example (fixed points at the modulation zero-crossing), we set $F_{a}\left(z_{0}\right)=F_{b}\left(z_{2}\right)=0$ and $R_{56}^{(a)}=R_{56}^{(b)}$. However, other manipulations are possible (see Section 7.3.1). Steady-state echo enabled harmonic generation (EEHG) may also be capable of driving SSMB at high harmonics (Section 7.3.2) [29].

\subsubsection{Analysis of Linearized Modulation}

For simulations we consider modulations $F_{a, b}=V_{a, b} \sin (k z)$. To study the zero-crossing fixed points analytically, we linearize the modulation, $F_{a, b}(z) \approx h_{a, b} z$, with $h_{a, b} \equiv V_{a, b} k$, and write the one turn map as a 


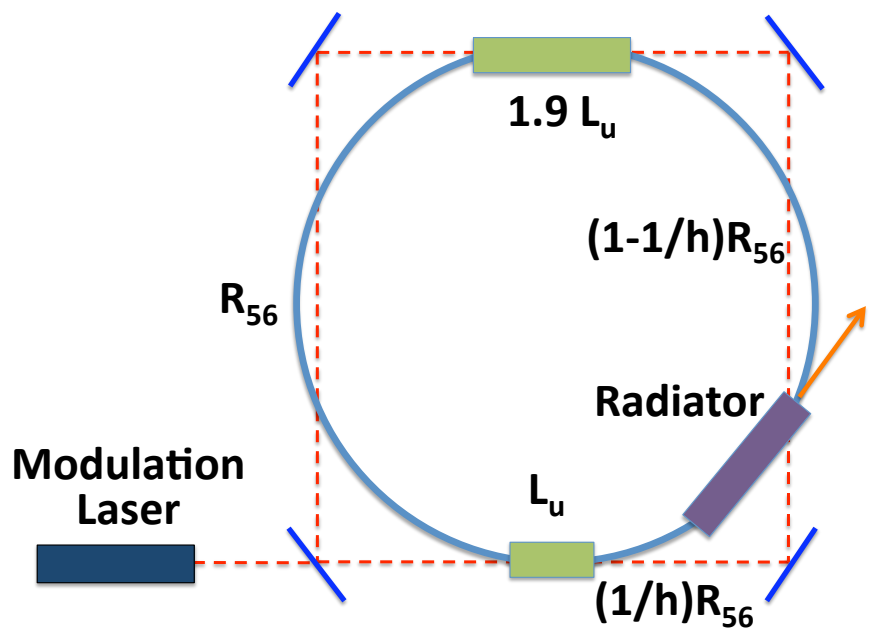

Figure 7.2: Example schematic for a two-stage system. A laser cavity and two undulators of length $L_{u}$ and $1.9 L_{u}$ modulate the electron beam at opposite ends of a storage ring. SSMB from the modulation and dispersion produces coherent light in a radiator. RF modules could replace the laser modulation to produce long wavelengths, and an additional radiator could be placed at the same distance in front of $L_{u}$.

matrix

$$
\begin{aligned}
M & =\left(\begin{array}{cc}
1 & R_{56}^{(b)} \\
0 & 1
\end{array}\right)\left(\begin{array}{cc}
1 & 0 \\
h_{b} & 1
\end{array}\right)\left(\begin{array}{cc}
1 & R_{56}^{(a)} \\
0 & 1
\end{array}\right)\left(\begin{array}{cc}
1 & 0 \\
h_{a} & 1
\end{array}\right) \\
& =\left(\begin{array}{cc}
1+s(2+(1+s) \eta) & \frac{s}{h_{a}}(2+s \eta) \\
h_{a}(1+(1+s) \eta) & 1+s \eta
\end{array}\right),
\end{aligned}
$$

with $R_{56}^{(a)}=R_{56}^{(b)} \equiv s / h_{a}$ and $\eta \equiv h_{b} / h_{a}$. The stability condition, $|\operatorname{Tr} M|<2$, constrains

$$
-4<2(s+s \eta)+s^{2} \eta<0 .
$$

However, we note that it is also possible to operate SSMB in the regime of $|\operatorname{Tr} M|>2$. The standard fixed point bifurcates into two fixed points which exchange particles each turn through the map. This approach could also generate SSMB [83].

We also write down an equilibrium bunch length using the Courant-Snyder parameters, $\left\langle z^{2}\right\rangle=\beta \varepsilon$ and $\left\langle\delta^{2}\right\rangle=\gamma \varepsilon$, yielding

$$
\left\langle z^{2}\right\rangle=-s \frac{2+s \eta}{(1+\eta+s \eta)} \frac{\left\langle\delta^{2}\right\rangle}{h_{a}^{2}},
$$

suggesting that $s \eta \sim-2$ can substantially reduce the island size, increasing the maximum possible harmonic. In reality, energy changes from damping and quantum excitation, combined with dispersion, will increase $\left\langle z^{2}\right\rangle$. However, in simulations we do observe moderate MB compression as $s \eta \rightarrow-2$. If the MB size is not a concern, it is also possible to modulate with a single stage SSMB scheme (Fig.7.3). 

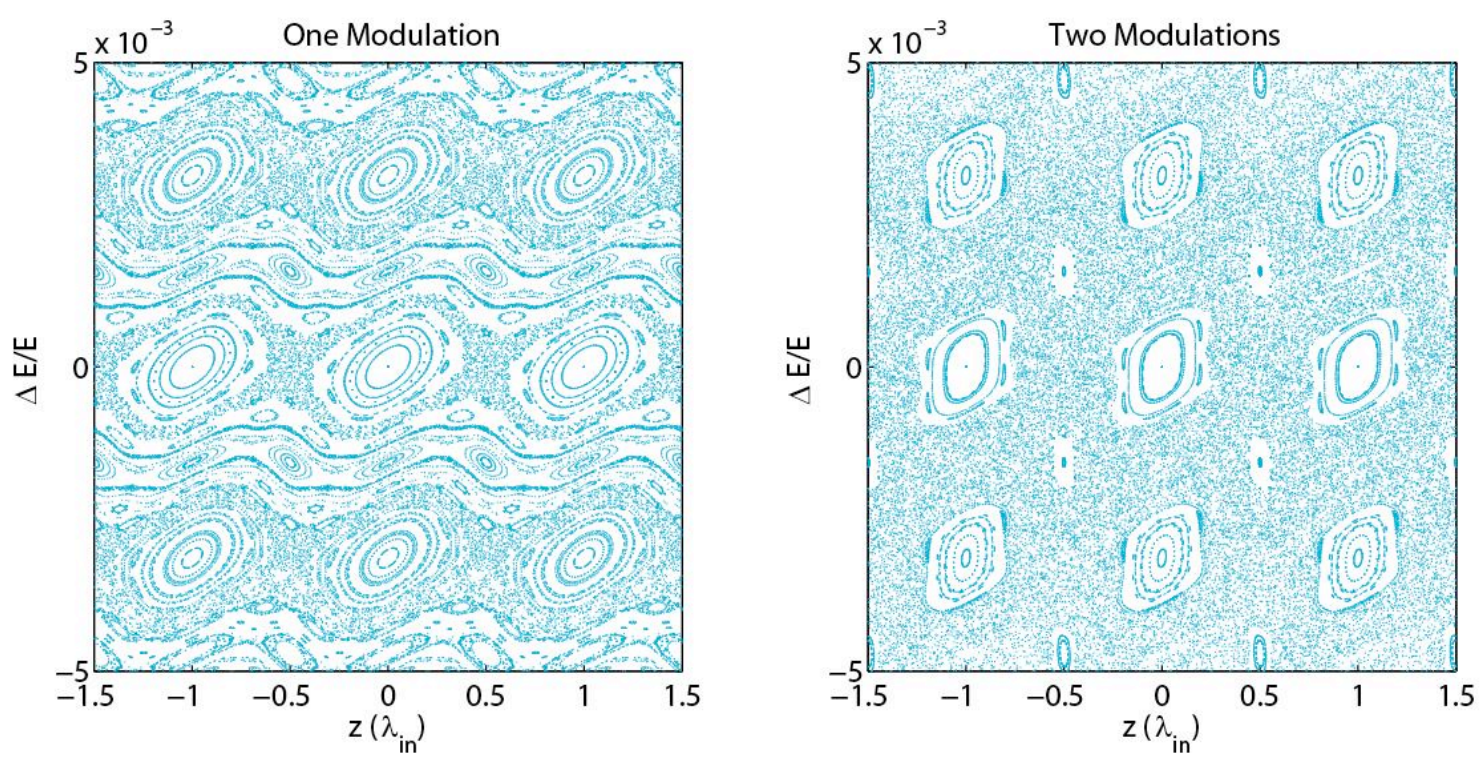

Figure 7.3: Phase space for harmonic SSMB, with one-stage modulation on left and two-stage modulation on right. The two-stage modulations provide a cleaner phase space with shorter stable regions, but the single modulation also creates stacked, periodic stable islands.

\subsection{Alternate Modes of SSMB}

We emphasize that the zero-crossing condition is not the only, nor necessarily even an optimal, method of modulation. Most generally, we are searching for solutions to the slippage condition, Eq. 7.2, that generate clean harmonic structure; we would like to find combinations of $R_{56}^{(a)}, R_{56}^{(b)}, V_{a}$, and $V_{b}$ that produce fixed points spaced evenly with respect to $\lambda_{\text {in }}$.

\subsubsection{Modulation-Canceling Slippage Condition}

In Section 7.2.1 we considered a simple example, constraining ourselves to the subset of Eq. 7.2 solutions with $p_{1}=p_{3}$ and $\bmod _{\lambda} z_{2}=\bmod _{\lambda} z_{0}=0$. Because of the latter constraint, we call this the 'zero-crossing' case. As a second example, we instead take the constraint $p_{1}=0$, which in turn implies $F_{a}=-F_{b}$. Because of the latter relation, we call this 'modulation-canceling;' the two modulations must offset to return the energy to its original state, $p_{f}=p_{0}$. Note that since we are no longer just looking at the zero-crossing, the linear expansion no longer holds for a sinusoidal modulation. If we consider a sawtooth waveform we may again assume a linear modulation at all fixed points.

We can write an equivalent transformation as that given in Eq. 7.3, though now with $h_{a}=h_{b} \equiv h$, and $R_{56}^{(a)} \neq R_{56}^{(b)}$. There is no constraint on the strength of $R_{56}^{(a)}$ (since we have $p_{1}=0$ ), but we have the same requirement as before that $R_{56}^{(b)}$ move each fixed point by an integer number of $\lambda_{\text {in }}$. Consequently, we again find the energy separation between islands, $\Delta p=2 n \pi k / R_{56}^{(b)}$. Whereas for zero-crossing the islands are all located at $\bmod _{\lambda} z_{0}=0$, we now find longitudinal separation $\Delta z=\Delta p / h$, and harmonic bunching after a full turn at $H=R_{56}^{(b)} h / 2 n \pi k$. We also find a new stability requirement,

$$
0<R_{56}^{(a)} R_{56}^{(b)} h^{2}<4 .
$$


So long as $R_{56}^{(a)}$ is small, we can find stable manipulations with high harmonics, $H$.

We do not concentrate on modulation-canceling because of the unequal distribution for the case of a sine wave modulation and the practical difficulty of producing a sawtooth manipulation (which would require multiple modulation wavelengths). However, we may easily simulate an example (Fig. 7.4).
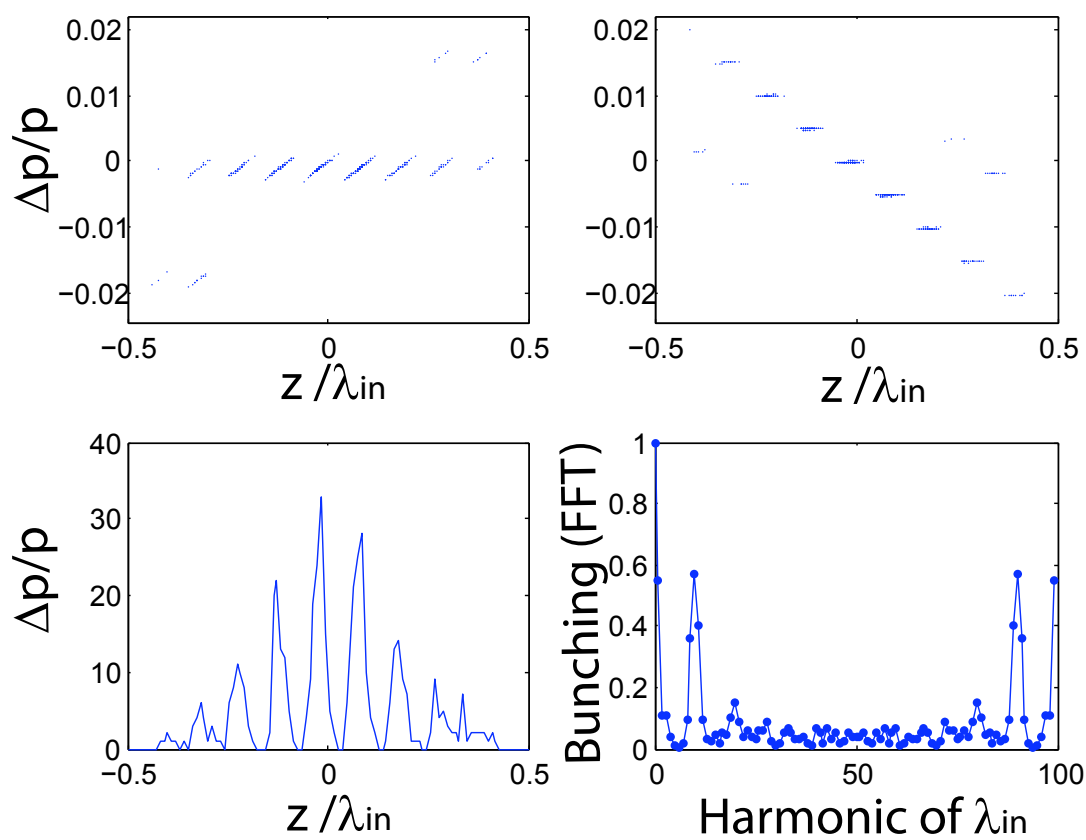

Figure 7.4: Example of an sawtooth manipulation to a coasting beam. Modulation has $V_{a}=V_{b}=0.05$, $R_{56}^{(a)}=1$ and $R_{56}^{(b)}=200$. After $2 \times 10^{5}$ loops, with damping of $\delta=10^{-4}$, unmodulated equilibrium energy spread of $10^{-4}$, and initial energy spread of $5 \%$, we find bunching at the 10th harmonic. Phase space of one wavelength is shown at upper left (after a full turn) and upper right (after $R_{56}^{(a)}$ ). The bunching profile is bottom left with the fourier transform bottom right.

\subsubsection{Echo}

\section{Steady State Echo Condition}

As a third example of a modulation method, we consider the EEHG modulation [29]. EEHG provided the initial motivation for SSMB, so we may be curious if we can implement EEHG directly as a steady state scheme. The EEHG process can be described as follows: an initial modulation and strong dispersive section filaments the beam. A second modulation and dispersive section then bunches each filament individually. The harmonic is determined by the separation of filaments (Fig. 7.5).

First we consider the case of a sawtooth modulation, $F=V_{a} \bmod _{\lambda}(z)$. We optimize the microbunching by producing a vertical slope in each sawtooth filament. We can solve for the sawtooth bunching condition from geometric arguments, by simply solving for the parameters that leave each segment with infinite slope 

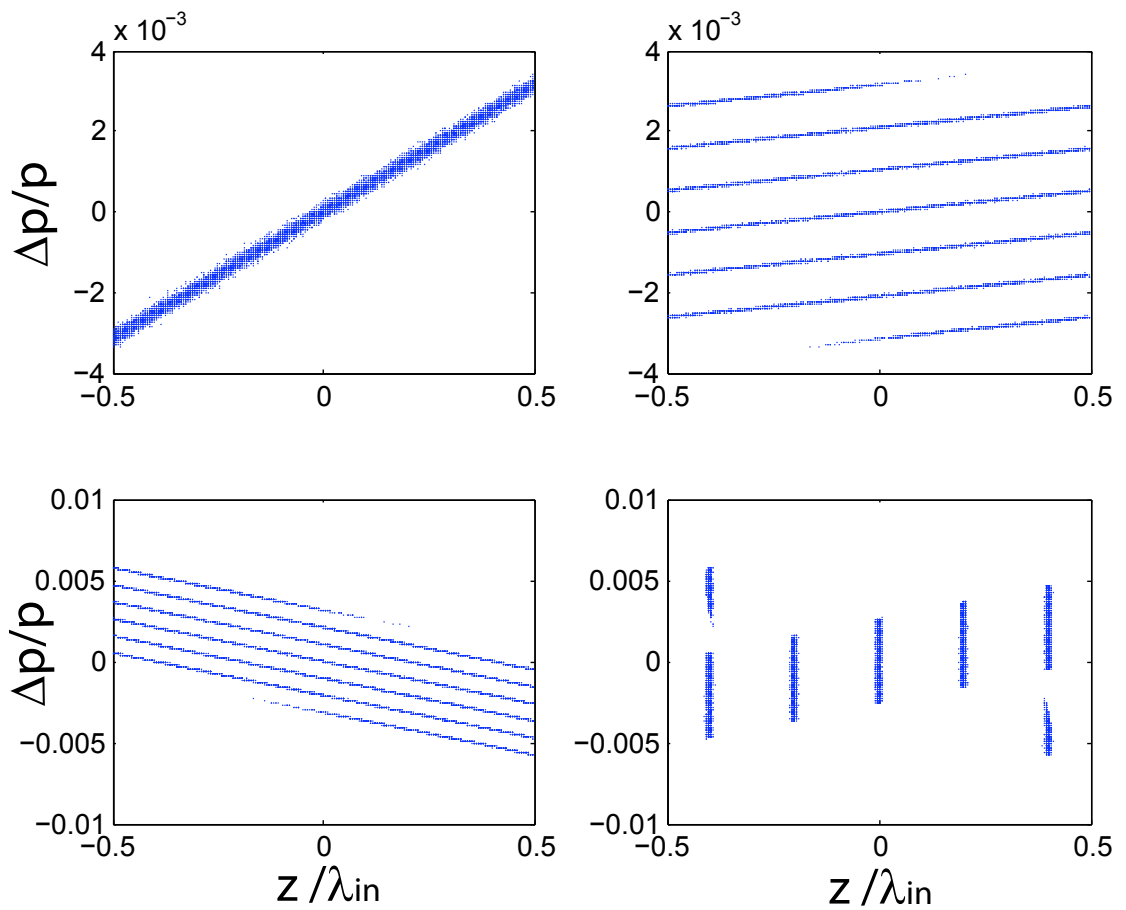

Figure 7.5: Illustration of the Echo mechanism for a sawtooth modulation. We have chosen modulations $V_{a}=-V_{b}=10 \sigma_{\delta}$ to make the scheme easier to follow. The first dispersive section filaments the beam. The second modulation and dispersive sections then individually bunch the filaments. The harmonic is determined by the vertical separation of filaments (upper right), which will become horizontal separation after the final dispersive section (bottom right). We may change the number of filaments independently of the harmonic number.

(upright in phase space). We can trace the slope through each section as

$$
\begin{aligned}
S_{1} & =V_{a} / \lambda_{\mathrm{in}} \\
S_{2} & =\frac{V_{a}}{\lambda_{\mathrm{in}}+R_{56}^{(a)} V_{a}} \\
S_{3} & =S_{2}+V_{b} / \lambda_{\mathrm{in}}=\frac{V_{a}}{\lambda_{\mathrm{in}}+R_{56}^{(a)} V_{a}}+\frac{V_{b}}{\lambda_{\mathrm{in}}},
\end{aligned}
$$

and we maximize bunching (final vertical segment) with

$$
R_{56}^{(b)}=-\frac{1}{S_{3}}=-\frac{\lambda_{\mathrm{in}}+R_{56}^{(a)} V_{a}}{V_{a}+V_{b}+R_{56}^{(a)} V_{a} V_{b} / \lambda_{\mathrm{in}}} .
$$

The spacing between the delta functions in the density profile, $\Delta z$, then determines the harmonic, $H=1 / \Delta z$. From the slopes calculated above, we find

$$
\Delta z=R_{56}^{(b)} \Delta p=R_{56}^{(b)} S_{2},
$$


where $\Delta p$ is the vertical separation of filaments after the third stage. Plugging in for $R_{56}^{(b)}$ and $S_{2}$, we find

$$
H=\frac{1}{\Delta z}=-\left(1+\frac{V_{b}}{V_{a}}+\frac{R_{56}^{(a)} V_{b}}{\lambda_{\text {in }}}\right) .
$$

If we consider $V_{b}=-V_{a}$, we have harmonic $H=R_{56}^{(a)} V_{b} / \lambda_{\text {in }}$.

We can apply our sawtooth model to a sine wave as well. While a single pass through EEHG has no general analytical solution for optimizing microbunching, we may guess that we optimize the steady state version by producing a vertical slope in each filament. Standing up the linear portion of the sine wave approximately maximizes bunching, so we will use the same condition as for the sawtooth, but with our new slope $V_{a} \rightarrow h_{a} \equiv V_{a} k$ and $V_{b} \rightarrow h_{b} \equiv V_{b} k$, immediately giving

$$
R_{56}^{(b)}=\frac{\lambda_{\text {in }}+R_{56}^{(a)} h_{a}}{h_{a}+h_{b}+R_{56}^{(a)} h_{a} h_{b} / \lambda_{\text {in }}} .
$$

We must be careful to allow for $h_{a} \rightarrow \pm h_{a}$, due to the two linear regions of a sine wave (phase 0 and $\pi$ ). For given values of $R_{56}^{(a)}$ and $h_{a}$, we now find two slopes in the second stage,

$$
S_{ \pm 2}=\frac{h_{a}}{R_{56}^{(a)} V_{a} \pm \lambda_{\text {in }}}
$$

The result is a more complicated harmonic structure. If we choose a value of $R_{56}^{(b)}$ to satisfy Eq. 7.8 for one of the initial slopes, we now find two harmonics corresponding to the two initial slopes $\pm h_{a}$, resulting in

$$
H_{ \pm}=\frac{1}{\Delta z}=\frac{1}{R_{56}^{(b)} S_{ \pm 2}}=H \frac{R_{56}^{(a)} h_{a} \pm \lambda_{\mathrm{in}}}{R_{56}^{(a)} h_{a}+\lambda_{\mathrm{in}}}
$$

where $H$ is the harmonic solution given for the sawtooth (Eq. 7.10). We then expect to find two harmonics, as evident in a single pass simulation (Fig.7.6). Had we used the optimal single pass bunching condition (which slightly over-bunches the modulation, see [29]), we would find enhancement of only one of the two harmonics.

\section{Steady State Implementation}

We would now like to see if it possible to use the echo manipulation in a steady state configuration. We can write down the linearized map

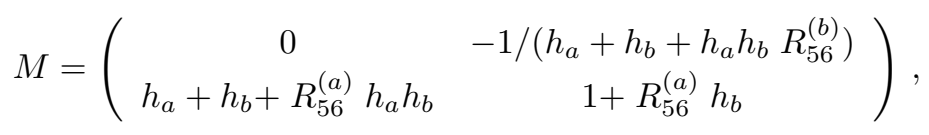

where we've used the bunching condition (Eq. 7.8) to remove the $R_{56}^{(b)}$ dependence and we've defined $\lambda_{\text {in }}=1$. The stability condition is given by

$$
-3<h_{b} R_{56}^{(a)}<1 .
$$

In general, we are interested in the case of producing multiple filaments from a single slope, so we must set $R_{56}^{(a)} h_{a} \gg \lambda_{\text {in }}$, or equivalently $\left|R_{56}^{(a)} h_{b} / \lambda_{\text {in }}\right| \gg\left|h_{b} / h_{a}\right|$. However, we can see a contradiction with our stability condition; in the multiple filament regime $\left(\left|h_{b} R_{56}^{(a)} / \lambda\right| \gg\left|h_{b} / h_{a}\right|\right)$ we cannot simultaneously have stability $\left(\left|h_{b} R_{56}^{(a)}\right|<3\right.$ ) and high harmonic, $H$ (Eqs.7.10,7.13). 

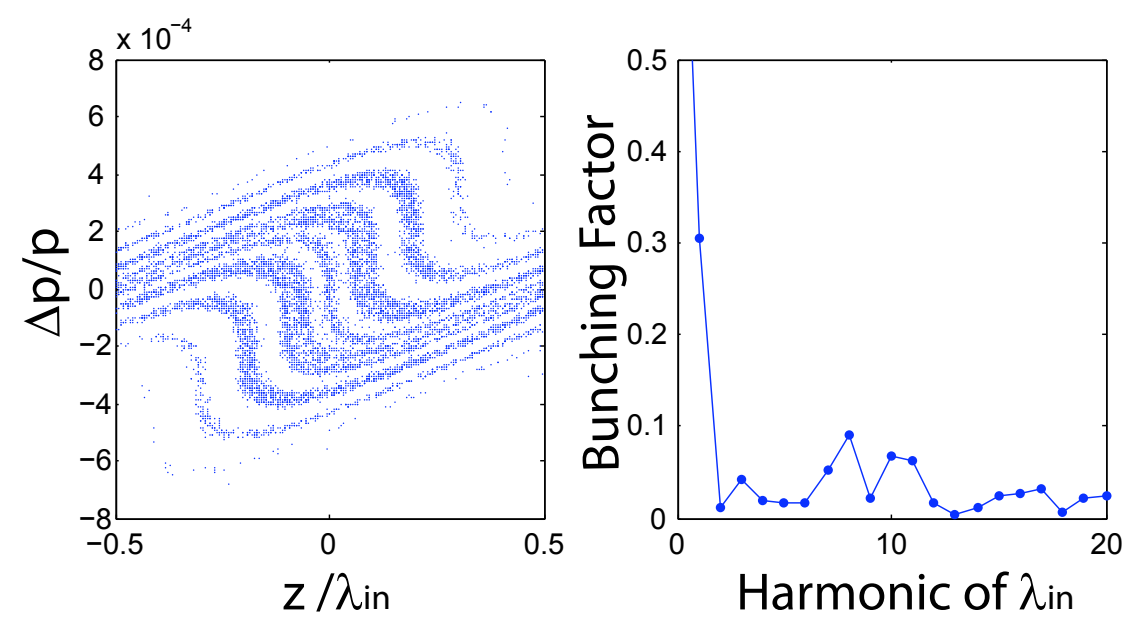

Figure 7.6: Illustration of the Echo mechanism for a sine modulation. We have chosen modulations $\bar{V}_{a}=$ $-\bar{V}_{b}=2 \sigma_{\delta}$, and set $R_{56}^{(a)}$ for the 10th harmonic. Because a sine has two zero crossings ( $h_{a}= \pm V k$ ), we will have two different solutions for $R_{56}^{(b)}$, giving two different harmonics $H_{ \pm}$. For the parameters above, we find $H_{+}=10$ and $H_{-} \approx 8$.

This analysis is valid only at the zero crossing and with the assumption that standing up the modulation maximizes SSMB. Neither assumption is critical for echo, so it may still be possible to implement the echo modulation as SSMB. We also may consider SSMB solutions that violate the stability condition as in [83].

\subsubsection{Long Wavelength Beating}

UV and x-ray radiation is the lifeblood of storage ring beamlines, so we have focused on generation of short wavelength SSMB with $\lambda_{\text {out }} \ll \lambda_{\text {in }}$. However, there is growing scientific and industrial interest in $\mathrm{THz}$ radiation, and storage rings in special configurations can generate these wavelengths as well [84]. Past work has demonstrated single pass microbunching with $\lambda_{\text {out }} \gg \lambda_{\text {in }}$ by beating two different input wavelengths, $\lambda_{\text {in } 1} \neq \lambda_{\text {in } 2}$ [85]. In an analogous fashion, we wish to generate long wavelength SSMB with $\lambda_{\text {out }} \gg \lambda_{\text {in }}$.

We consider modulation with two wavelengths related by $\lambda_{\mathrm{in} 2}=[b /(b-1)] \lambda_{\mathrm{in} 1}$. The frequency beating results in periodicity at an output wavelength $\lambda_{\text {out }}=b \lambda_{\text {in } 1}$, with the potential to set $b \gg 1$.

Frequency beating can drive SSMB in either one or two stages (Fig. 7.7). First we consider a two stage SSMB, with separate modulation and dispersive regions for each wavelength. With two modulations, fixed points exist only at the stable phases of both modulations. Consequently, we expect particles to survive only in regions where the modulation phases match. Elsewhere, where the phases do not match, one modulation always ejects particles from the stable points of the other modulation. The result is sets of fixed points separated by the beat wavelength, $\lambda_{\text {out }}$.

Alternatively, we can implement frequency beating with a single stage setup. In this case stable fixed points survive across $\lambda_{\text {out }}$. However, if we rely on the modulation to replace the synchrotron radiation energy loss, $\delta$, fixed points only survive where the combined modulation, $V_{a} \cos \left(k_{a} z\right)+V_{b} \cos \left(k_{b} z\right)$, is larger than $\delta$. For $\delta \lesssim V_{a}+V_{b}$, stable fixed points again remain only where the phases overlap, near $\bmod _{\lambda \text { out }} z=0$, resulting in SSMB at the beat wavelength, $\lambda_{\text {out }}$. We compare the results of single and two stage frequency 


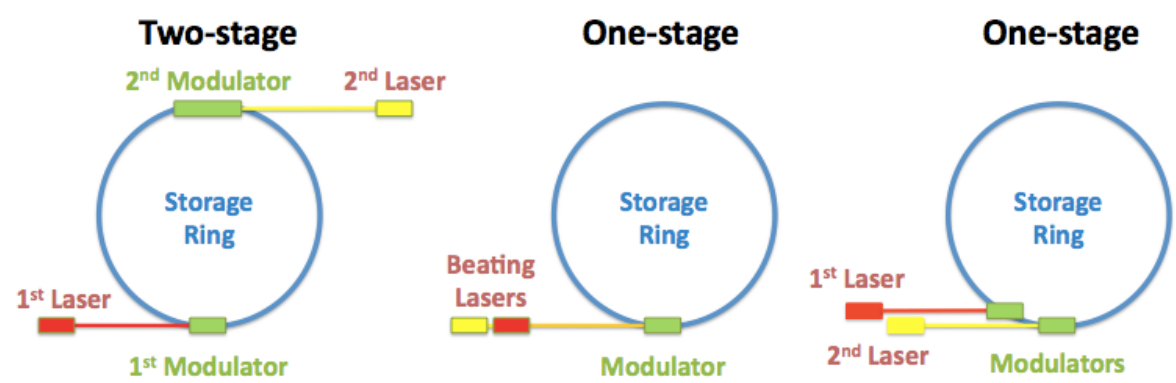

Figure 7.7: Frequency-beating drives SSMB in multiple configurations. At left, modulations are in separate stages, and stable fixed points survive only where the phases overlap. At center, beating the laser prior to modulation can also drive SSMB if the electrons rely on the modulation to replace energy lost to synchrotron radiation. At right, the one-stage scheme is identical to a two-stage scheme with no dispersion between modulations.

beating in Figs. 7.8 and 7.9.

As a side note, the one stage beating scheme provides a peculiar method for chirping the beam, described in Fig. 7.10. It is possible to compress the beam using this chirp by using a large dispersive strength relative to the $R_{56}$ of the manipulation.

\subsubsection{Linearized RF bucket}

We can implement the long wavelength beating scheme as a CW source in a small, low-energy ring as in the EUV case. If we use the modulation to accelerate the electrons as well, we can do away with the RF modules entirely. Alternatively, we may consider a pulsed version, with RF buckets to accelerate the particles (allowing lower power modulation lasers). To provide a uniform acceleration across the entire bunched beam, we require an RF wave that is approximately constant over a length much longer than $\lambda_{\text {out }}$. However, with radiation as long as $\lambda_{\text {out }} \sim 1 \mathrm{~mm}$, this requirement may fail for RF of wavelength $\lambda_{\mathrm{rf}} \sim 100 \mathrm{~mm}$.

Instead of using a single RF modulation, we consider two cavities, prime and double-prime, with different wavevectors, $k_{\mathrm{rf}}{ }^{\prime \prime} \neq k_{\mathrm{rf}}{ }^{\prime}$, and arbitrary amplitudes and phases $V_{\mathrm{rf}}{ }^{\prime}, V_{\mathrm{rf}}{ }^{\prime \prime}, \alpha_{\mathrm{rf}}{ }^{\prime}, \alpha_{\mathrm{rf}}{ }^{\prime \prime}$. We have four constraints: the total modulation is equal to the damping $(\delta)$, the linear term must be smaller than some desired slope, $S_{\mathrm{rf}}$, and the quadratic and cubic terms should disappear, to maximize the linear region. Expanding the sinusoidal functions around the phases $\alpha_{\mathrm{rf}}{ }^{\prime}, \alpha_{\mathrm{rf}}{ }^{\prime \prime}$, we write the constraints as

$$
\begin{aligned}
V_{\mathrm{rf}}{ }^{\prime} \cos \left(\alpha_{\mathrm{rf}}{ }^{\prime}\right)+V_{\mathrm{rf}}{ }^{\prime \prime} \cos \left(\alpha_{\mathrm{rf}}{ }^{\prime \prime}\right) & =\delta \\
V_{\mathrm{rf}}{ }^{\prime} k_{\mathrm{rf}}{ }^{\prime} \sin \left(\alpha_{\mathrm{rf}}{ }^{\prime}\right)+V_{\mathrm{rf}}{ }^{\prime \prime} k_{\mathrm{rf}}{ }^{\prime \prime} \sin \left(\alpha_{\mathrm{rf}}{ }^{\prime \prime}\right) & =2 S \\
V_{\mathrm{rf}}{ }^{\prime} k_{\mathrm{rf}}{ }^{2} \cos \left(\alpha_{\mathrm{rf}}{ }^{\prime}\right)+V_{\mathrm{rf}}{ }^{\prime \prime} k_{\mathrm{rf}}{ }^{\prime 2} \cos \left(\alpha_{\mathrm{rf}}{ }^{\prime \prime}\right) & =0 \\
V_{\mathrm{rf}}{ }^{\prime} k_{\mathrm{rf}}{ }^{3} \sin \left(\alpha_{\mathrm{rf}}{ }^{\prime}\right)+V_{\mathrm{rf}}{ }^{\prime \prime} k_{\mathrm{rf}}{ }^{\prime 3} \sin \left(\alpha_{\mathrm{rf}}{ }^{\prime \prime}\right) & =0,
\end{aligned}
$$

and find relations 

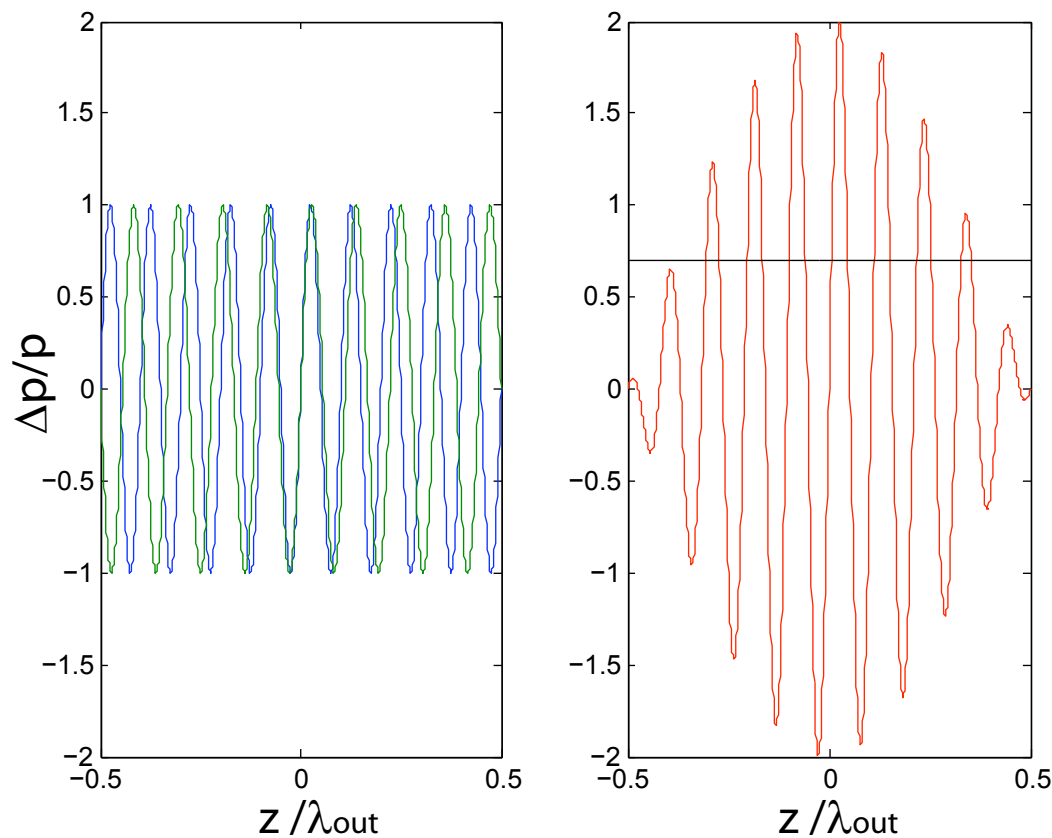

Figure 7.8: Example of a long wavelength beating modulation with $b=10$. On the left, the two stage modulation affects particles throughout the beam, but stable fixed points can only survive near the center where the phases overlap. On the right, in a one stage modulation the two lasers beat together before interacting with the beam, and the modulation has little effect where the phases cancel. The one stage modulation still results in bunching when the combined modulation amplitude is less than the radiation loss (black line). Because the electrons rely on the modulation to replace energy lost to radiation, stable islands only survive in the center where the modulation is greater than the radiation loss.

$$
\begin{aligned}
& \tan \left(\alpha_{\mathrm{rf}}{ }^{\prime}\right)=\frac{2 S}{k_{\mathrm{rf}}{ }^{\prime} \delta}, \tan \left(\alpha_{\mathrm{rf}}{ }^{\prime \prime}\right)=\frac{2 S}{k_{\mathrm{rf}}{ }^{\prime \prime} \delta} \\
& V_{\mathrm{rf}}{ }^{\prime}=\frac{\sqrt{\delta^{2}+\left(2 S / k_{\mathrm{rf}}{ }^{\prime}\right)^{2}}}{1-k_{\mathrm{rf}}{ }^{2} / k_{\mathrm{rf}}{ }^{\prime 2}}, V_{\mathrm{rf}}{ }^{\prime \prime}=\frac{\sqrt{\delta^{2}+\left(2 S / k_{\mathrm{rf}}{ }^{\prime \prime}\right)^{2}}}{1-{k_{\mathrm{rf}}}^{\prime 2} / k_{\mathrm{rf}}{ }^{2}} \text {. }
\end{aligned}
$$

After choosing the frequencies, $k_{\mathrm{rf}}{ }^{\prime}, k_{\mathrm{rf}}{ }^{\prime \prime}$, slope, $S$, and damping, $\delta$, we can solve for the corresponding phases and amplitudes to optimize the RF acceleration for SSMB.

\subsubsection{Hamiltonian View}

As an alternative view of the SSMB process, we may consider the modulation process as a modification of the unmodulated Hamiltonian. For example, we can picture the net effect of the RF bucket as generating a wide valley in the Hamiltonian that traps particles in a single group at the center of the valley. In this view, the roll of SSMB is to add a modulation to the floor of the Hamiltonian; the resulting washboard structure then divides the large single group into periodic microbunches. In this sense, the particles are not a strict steady-state solution (on an individual particle basis). Rather, the statistical distribution of particles due to 

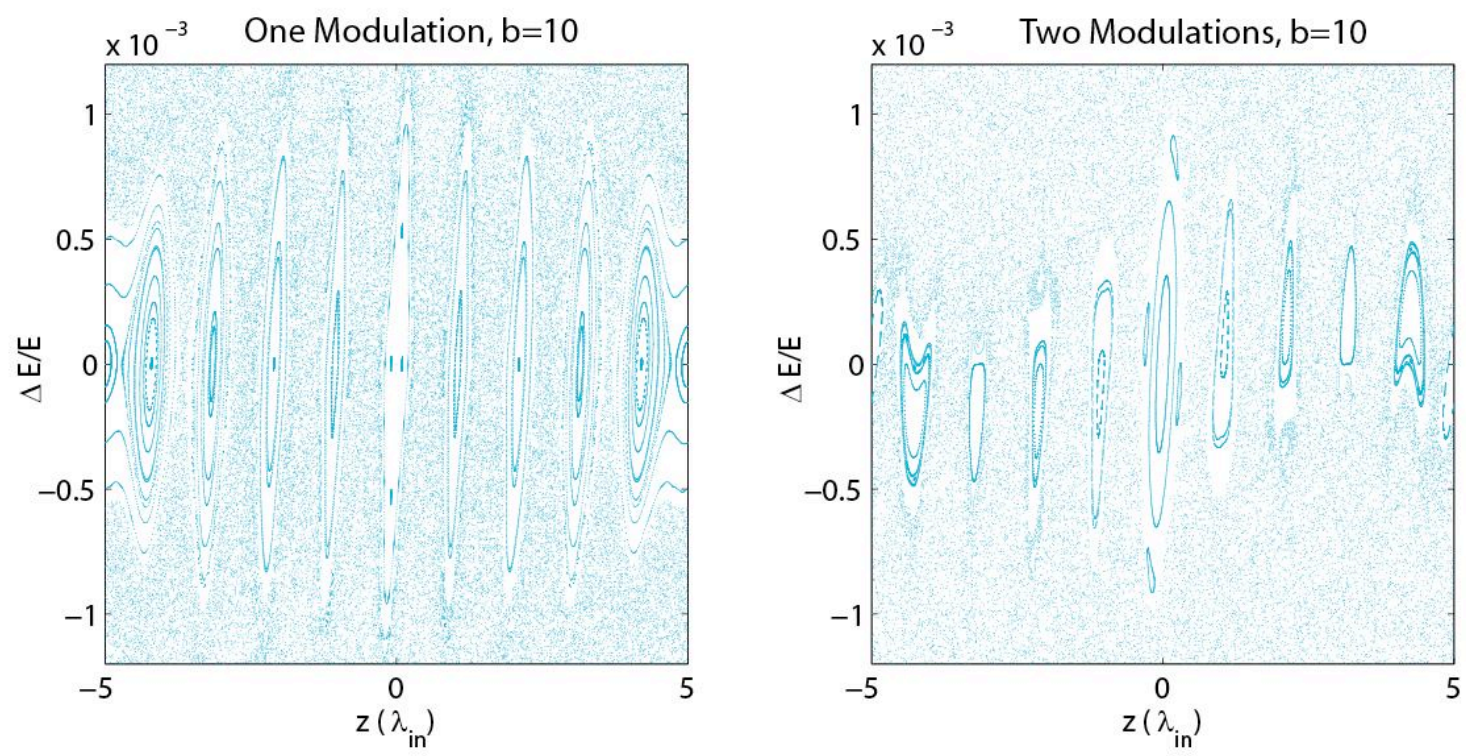

Figure 7.9: Phase space for frequency beating (with $b=10$ ) for both two-stage modulation (left) and onestage modulation (right). For two-stage modulations, the largest stable regions exist where the phases overlap. In the one-stage case, we find phase space stable islands throughout $\lambda_{\text {out }}=b \lambda_{\text {in }}$, but radiative energy loss removes the side islands.

the washboard Hamiltonian leads to strong bunching factors at short wavelengths, and these bunching factors exist at steady state. This picture may help develop new algorithms for implementing SSMB.

\subsection{Example Radiation Sources from SSMB}

\subsubsection{EUV Source}

For each turn around the ring, the SSMB beam passes through a radiator (e.g. an undulator). Matching the SSMB wavelength to the resonant wavelength of an undulator strongly amplifies the radiation brightness. As a first example, we consider the feasibility of SSMB at the EUV lithography standard, $13.5 \mathrm{~nm}$. To produce a stable, high power $\mathrm{CW}$ modulation, we overlay the storage ring with a laser cavity and two modulation undulators, with a third undulator to extract radiation (Fig. 7.2). In general, a laser modulation does not involve any average energy transfer. However, in the absence of an RF bucket, the modulation process must replace the radiative energy loss.

We illustrate the concept with a simulation that tracks the 4-D particle coordinates $\left(z, p, x, x^{\prime}\right)$ through a large number of passes around the ring. The simulation includes both energy loss $(\delta)$ and quantum fluctuations $(\epsilon)$ from synchrotron radiation, as well as first and second order momentum compaction $\left(R_{56}, T_{566}\right)$ and transverse transport elements $\left(R_{51}, R_{52}\right)$. We model jitter errors by shifting the modulation relative to the particles on a turn-by-turn basis. We assume an initially flat-top longitudinal distribution with Gaussian transverse profile of radius $\sigma_{x}, \sigma_{x^{\prime}}$, though we note the two synchrotron radiation terms, $\delta$ and $\epsilon$, will determine the equilibrium unmodulated energy spread $\left(\sigma_{\delta}\right)$. Periodic boundary conditions allow for slippage 


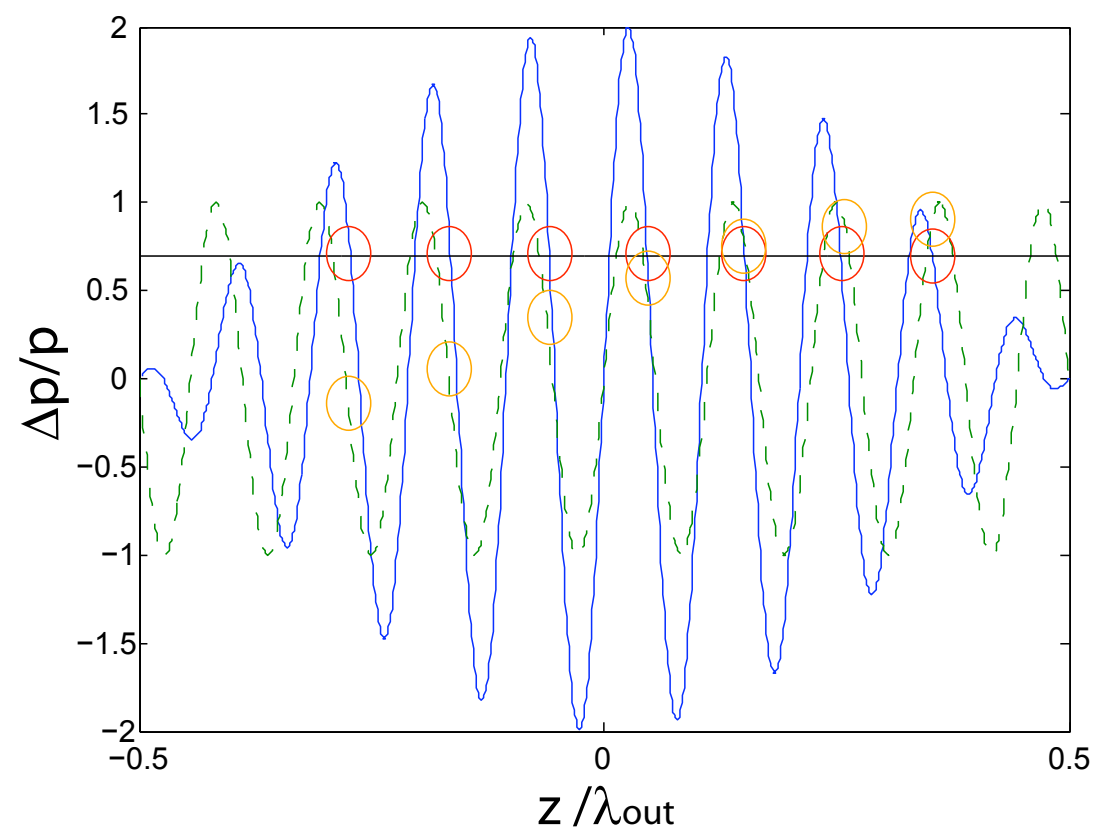

Figure 7.10: In a system without RF, the modulation both drives SSMB and replaces the energy loss due to synchrotron radiation. The stable islands of the one-stage modulation are centered at the intersection of the synchrotron radiation (black line) and the combined beating modulation (blue line), with the intersection points highlighted by the red circles. Modulating with just one of the two beating wavelengths (green, dotted line) will modulate the stable islands by the level of the orange circles. We can see that this modulation chirps the stable islands, because of a relative phase slippage. A dispersive region can then compress the bunches. The sine wave shown (green) has the larger of the two beating wavelengths. Were we to have chosen the shorter wavelength, the chirp would have the opposite sign.

across many wavelengths.

A laser with power $P_{L}$ will generate a modulation amplitude of

$$
V_{a}=\sqrt{\frac{P_{L}}{P_{0}}} \frac{K L_{u}}{\gamma_{0} \sigma_{L}} A_{\mathrm{JJ}},
$$

with $P_{0}=8.7 \mathrm{GW}$ and Bessel factor $A_{J J} \equiv J_{0}(Q)-J_{1}(Q), Q \equiv K^{2} /\left(4+2 K^{2}\right)$ for a planar undulator. With undulator parameter $K=1$ and lengths $L_{u}=1,1.8 \mathrm{~m}$ for the two modulations, we need power of around $P_{L}=10 \mathrm{MW}$ focused to roughly $\sigma_{L}=100 \mu \mathrm{m}$ to produce $V_{a}=225 \mathrm{keV}$.

For a $13.5 \mathrm{~nm}$ source, we simulate a $500 \mathrm{MeV}$ ring, modulated at $\lambda_{\mathrm{in}}=200 \mathrm{~nm}$ and look for SSMB at the 15th harmonic (Tab. 7.1). To maintain the short wavelength SSMB we require a large energy aperture $( \pm 6 \%)$ and a laser cavity with a strong $50 \mathrm{MW}$ stored power, though only with a few $\mathrm{kW}$ of $\mathrm{CW}$ input power. Stability of the laser is assumed to be $0.005^{\circ}$ in phase and $0.005 \%$ in amplitude. Choosing $\eta=1.9$, after 10 million turns we then observe trapping of $\sim 15 \%$ of the particles with strong SSMB (Fig. 7.11). (A large ring with weak bends may permit decreased laser power and energy aperture, but we have not explored ring optimization.)

From Eq. 27 in Ref.[86], we can estimate the power of a thin electron beam $\left(k_{r} \sigma_{x}^{2} / L_{u} \gg 1\right)$ from a 
Table 7.1: Example Parameters

\begin{tabular}{|l|c|c|c|}
\hline & EUV & THz & Pump-probe \\
\hline Rad. wavelength $\left(\lambda_{\text {out }}\right)$ & $13.5 \mathrm{~nm}$ & $500 \mu \mathrm{m}$ & NA \\
\hline Seed wavelength $\left(\lambda_{\text {in }}\right)$ & $200 \mathrm{~nm}$ & $2.0,2.008 \mu \mathrm{m}$ & $1 \mathrm{~cm}$ \\
\hline Modulation amp. $\left(V_{a}\right)$ & $1 \mathrm{MeV}$ & $350 \mathrm{keV}$ & $15 \mathrm{MeV}$ \\
\hline Dispersion $\left(R_{a}+R_{b}\right)$ & $30 \mu \mathrm{m}$ & $0.9 \mathrm{~mm}$ & $0.6 \mathrm{~m}$ \\
\hline Beam energy $(E)$ & $500 \mathrm{MeV}$ & $500 \mathrm{MeV}$ & $3 \mathrm{GeV}$ \\
\hline Damp. decrement $(\delta)$ & $5 \times 10^{-6}$ & $5 \times 10^{-6}$ & $3 \times 10^{-4}$ \\
\hline Equil. $E$ spread $\left(\sigma_{\delta}\right)$ & $3 \times 10^{-4}$ & $5 \times 10^{-4}$ & $1 \times 10^{-3}$ \\
\hline
\end{tabular}

bunched beam as

$$
W=W_{b} \frac{\pi B^{2}}{2} \frac{I}{\gamma I_{A}} \frac{K^{2}}{2+K^{2}} N_{u} A_{\mathrm{JJ}}
$$

with electron beam power $W_{b}$, bunching factor $B$, electron current, $I$, Alfvén current, $I_{A}$, and number of undulator periods, $N_{u}$. Assuming a $450 \mathrm{MeV}$ beam, with $500 \mathrm{~mA}$ current and $10 \mu \mathrm{m}$ radius, a $2 \mathrm{~m}$ undulator with $8 \mathrm{~mm}$ period can deliver nearly $800 \mathrm{~W}$ of continuous power. While many of these parameters (in particular current, momentum compaction, laser stability, and unmodulated equilibrium energy spreads) are at or beyond the current technological limit, a less aggressive design may still be of interest to the lithography community. There is also trade off in various parameters (e.g., we can double the energy spread at the cost of doubling the modulation amplitude). Technological advances could allow for wavelengths in the soft X-ray region.
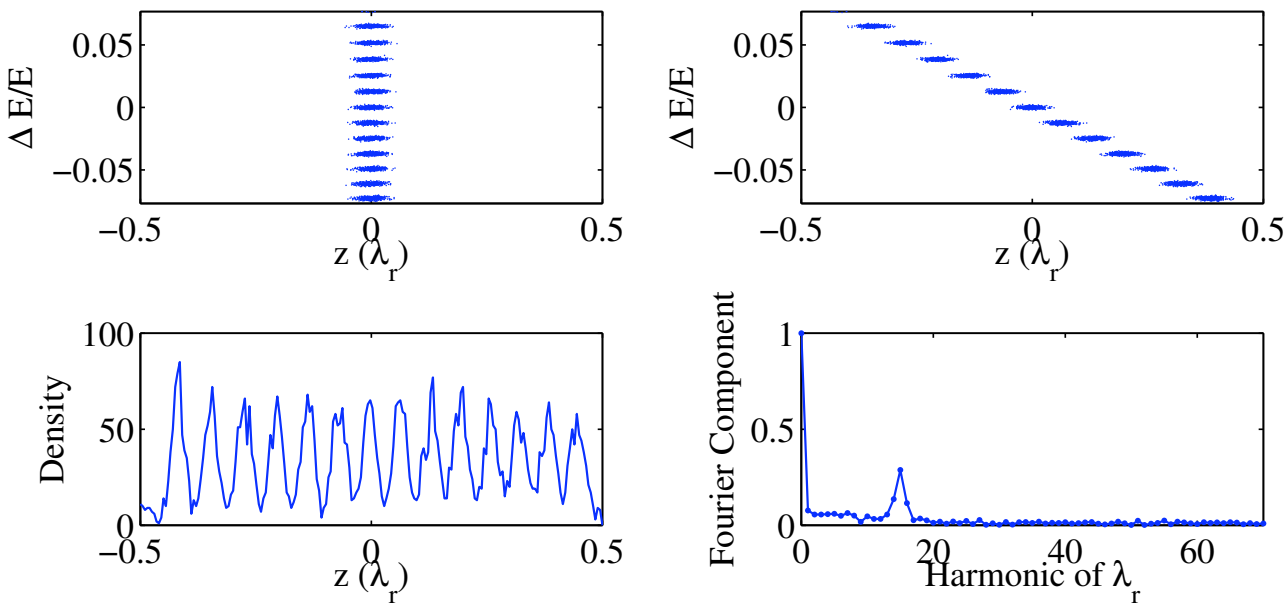

Figure 7.11: Simulation of a two-stage manipulation to produce SSMB. After 10 million revolutions, we find bunches stacked at steady-state in energy space (top left). After an additional dispersive section of $R_{56} / 15$, we find SSMB at the 15th harmonic (top right). The density profile is shown bottom left, with the Fourier transform bottom right. 


\subsubsection{THz and Pump Probe Sources}

We also provide two illustrations of long wavelength SSMB. Rings operating with low momentum compaction factors have produced coherent radiation from isolated, short pulses with $\sigma_{z}<\lambda_{\text {out }}[84,87]$. With a coherent train of pulses from frequency-beating SSMB, we propose to generate high average power $\mathrm{THz}$ radiation. As an example, we simulate $\lambda_{\text {in } 1}=2 \mu \mathrm{m}$ and multiplying factor $b=250$ for a $500 \mathrm{MeV}$ ring with realistic damping and energy spread (Tab. 7.1). With laser stability of $0.01^{\circ}$ in phase and $.01 \%$ in amplitude, we find strong bunching at $\lambda_{\text {out }}=500 \mu \mathrm{m}$. Better laser stability would permit lower ring energy.

It is also possible to modulate with RF and use harmonic SSMB to reach THz. The long wavelength modulation allows for much looser stability requirements, making practical implementation easier. As an example, Ref. [83] considers the possibility of operating above the fixed point bifurcation to produce $\mathrm{THz}$ from an RF modulation.

Alternatively, RF modulations could be used to drive pump-probe experiments, which use two pulses, separated by a variable time delay, to study dynamical systems. Using SSMB to create two microbunches, a variable dispersive strength between the modulation and the radiator would change the time delay between pulses. While not coherent, an SSMB pump-probe at a multi-GeV ring (Tab. 7.1) would radiate in the X-ray regime. As a side benefit, the difference in energy of the two bunches leads to slightly different radiation wavelengths. Such 'two-color' output helps users distinguish the pump and probe pulses. We note that two-color double pulses have previously been observed from $\alpha$ buckets [88].

\subsection{Technical Challenges}

Operating modes with low momentum compaction factor produce short bunches, creating large ratios of peak current to average current. To prevent the amplified peak currents from driving longitudinal instabilities, the average currents are typically very low $[84,45,89,90]$. However, the SSMB process spreads particles evenly across many microbunches in each bucket, so that the ratio of peak to average current is approximately the harmonic number, $H$, or smaller (Fig. 7.11). We then expect the SSMB process to support relatively large average currents despite the small momentum compaction factor.

SSMB is relatively immune to static errors in laser phase $(\phi)$, modulation amplitude $(V)$, and dispersion $\left(R_{56}\right)$ between the two stages. For steady-state solutions, static errors only shift the equilibrium fixed points. From the EUV simulation we find $\Delta \phi<5^{\circ}, \Delta V<5 \%, \Delta R_{56}<1 \%$ and $T_{566} \lesssim R_{56}$ do not inhibit SSMB.

However, SSMB is potentially sensitive to stability errors, especially for long damping times. Small jitters in timing between the laser and electron bunch may accumulate and smear out the structure, requiring high phase and amplitude stability. If the laser cavity cannot achieve the required stability, the electron bunch itself could serve as the modulation source, intrinsically locking the electrons to the modulation. Self-modulation is possible for manipulations which generate bunching at $\lambda_{\text {in }}$; in Fig. 7.11, we see nearly $100 \%$ bunching at $\lambda_{\text {in }}$ at the modulation point (top left). However, self-modulation will increase the amplitude jitter.

The tightest constraints come from transverse transfer elements, $R_{51}, R_{52}$, which will de-trap particles and lengthen the SSMB unless $R_{51} \sigma_{x}, R_{52} \sigma_{x^{\prime}} \ll \lambda_{\text {in }}, \lambda_{\text {out }}$. Particles with the largest $x, x^{\prime}$ values are lost, which may result in shrinking of $\sigma_{x}, \sigma_{x^{\prime}}$. It is interesting to note that keeping the transverse coordinates fixed (i.e., integer transverse tune, $\nu$ ) adjusts the slippage condition (Eq. 7.2) by $R_{56} p \rightarrow R_{56} p+R_{51} x+R_{52} x^{\prime}$. The constraints on $R_{51} \sigma_{x}$ and $R_{52} \sigma_{x^{\prime}}$ then relax, but practically it would be difficult to operate a ring close 
to an integer tune.

\subsection{Conclusion}

We present a mechanism for producing SSMB in a storage ring. The combination of dispersion and modulation regions produces fixed points (and thus SSMB) at harmonics or multiples of the modulation wavelength. We acknowledge that preserving fine SSMB will be challenging at short wavelengths, and briefly discuss some potential technical requirements. A proof of principle may be possible at long wavelengths, and we will address practical design issues in future studies.

\subsection{Acknowledgments}

We would like to thank J. Corbett, V. Dolgashev, Z. Huang, Y. Jiao, Y. Nosochkov, R. Ruth, M. Sjostrom, and D. Xiang for many helpful discussions. This work was supported by U.S. DOE Contract No. DE-AC02$76 \mathrm{SF} 00515$. 


\section{Chapter 8}

\section{Numerical Calculation of Longitudinal Space-Charge in an Undulator}

\subsection{Introduction}

Current enhanced SASE (eSASE) was proposed as a method to improve peak power, timing, and pulse length control in SASE FELs [39]. The scheme uses an optical laser and dispersive region to create sub-micron current spikes in the electron bunch. The current spikes lase more efficiently than the lower current regions, providing short bursts of FEL radiation synched to an optical laser pulse (i.e. coherent control). Coherent control is particularly useful for pump-probe experiments with X-ray FELs, and eSASE has been proposed as an upgrade to LCLS [40]. However, the sharp current spikes also exacerbate longitudinal space charge (LSC) induced energy spread in the electron beam. While LSC is generally suppressed in electron beams by the relativistic Lorentz factor, $\gamma=1 / \sqrt{1-\beta^{2}}$, Geloni et al. showed that the suppression is more accurately given by $\gamma_{z}=1 / \sqrt{1-\beta_{z}^{2}}$, with average longitudinal velocity, $\beta_{z}$ [41]. In an undulator, $\gamma_{z} \ll \gamma$, and the LSC may have a strong effect on the FEL process. The motivation for this note was to confirm Geloni's result with a numerical integration of the retarded electromagnetic potentials.

In this note we use a 2-D numerical approach to calculate the longitudinal self-field of an electron bunch. In the first section, we describe the approach and derive the longitudinal component of the interaction for arbitrary motion. We express the equations in polar coordinates to facilitate a numerical code written in MATLAB. We then solve the equations for several simple examples, including a bunch traveling in uniform, circular and sinusoidal motion. We use these examples to benchmark the code against known solutions, and then finally confirm Geloni's expression for LSC of a Gaussian bunch in an undulator.

\subsection{Field Components}

We describe the electron beam with a cold fluid approximation. In this approximation, a fluid element located at point $\boldsymbol{r}$ of the beam is represented by the charge density $\rho(\boldsymbol{r}, t)$ and the velocity of motion $\boldsymbol{v}(\boldsymbol{r}, t)$, while neglecting the finite emittance of the beam. The density and velocity functions are considered given, determined by the initial conditions and the leading magnetic field of the system. The self field of the beam 
is considered as a perturbation.

\subsubsection{Scalar Potential}

The electric field of the bunch is given by the following equation

$$
\boldsymbol{E}(\boldsymbol{r}, t)=-\boldsymbol{\nabla}_{r} \phi-\frac{\partial \boldsymbol{A}}{\partial t}
$$

with scalar and vector potentials

$$
\begin{gathered}
\phi(\boldsymbol{r}, t)=\frac{1}{4 \pi \epsilon_{0}} \int d r^{\prime} \frac{\rho\left(\boldsymbol{r}^{\prime}, t_{\mathrm{ret}}\right)}{\left|\boldsymbol{r}^{\prime}-\boldsymbol{r}\right|}, \\
\boldsymbol{A}(\boldsymbol{r}, t)=\frac{\mu_{0}}{4 \pi} \int d r^{\prime} \frac{\boldsymbol{j}\left(\boldsymbol{r}^{\prime}, t_{\mathrm{ret}}\right)}{\left|\boldsymbol{r}^{\prime}-\boldsymbol{r}\right|},
\end{gathered}
$$

where $t_{\text {ret }}=t-\left|\boldsymbol{r}-\boldsymbol{r}^{\prime}\right| / c$, and $\nabla_{r}$ is the gradient operator applied to the coordinates of the vector $\boldsymbol{r}$. In our approach, the current density, $\boldsymbol{j}$, is equal to the product of the charge density and the velocity, $\boldsymbol{j}(\boldsymbol{r}, t)=$ $\boldsymbol{v}(\boldsymbol{r}, t) \rho(\boldsymbol{r}, t)$.

Our goal will be to calculate the change in energy of a particle in the beam

$$
\frac{d W}{d t}=q(\boldsymbol{v} \cdot \boldsymbol{E})
$$

To find the contribution from the scalar potential to the electric field, we evaluate

$$
\begin{aligned}
\nabla_{r} \phi & =\frac{1}{4 \pi \epsilon_{0}} \int d r^{\prime}\left[\frac{\nabla_{r} \rho\left(\boldsymbol{r}^{\prime}, t_{\mathrm{ret}}\right)}{\left|\boldsymbol{r}^{\prime}-\boldsymbol{r}\right|}+\rho\left(\boldsymbol{r}^{\prime}, t_{\mathrm{ret}}\right) \nabla_{r} \frac{1}{\left|\boldsymbol{r}^{\prime}-\boldsymbol{r}\right|}\right] \\
& =\frac{1}{4 \pi \epsilon_{0}} \int d r^{\prime}\left[\frac{\nabla_{r} \rho\left(\boldsymbol{r}^{\prime}, t_{\mathrm{ret}}\right)}{\left|\boldsymbol{r}^{\prime}-\boldsymbol{r}\right|}-\rho\left(\boldsymbol{r}^{\prime}, t_{\mathrm{ret}}\right) \nabla_{\boldsymbol{r}^{\prime}} \frac{1}{\left|\boldsymbol{r}^{\prime}-\boldsymbol{r}\right|}\right],
\end{aligned}
$$

where in the second line we have changed to the gradient operator $\nabla_{r^{\prime}}$ with respect to the vector $\boldsymbol{r}^{\prime}$. Using the chain rule and integrating by parts gives

$$
\begin{aligned}
\nabla_{r} \phi & =\frac{1}{4 \pi \epsilon_{0}} \int d r^{\prime}\left[\frac{\nabla_{r} \rho\left(\boldsymbol{r}^{\prime}, t_{\mathrm{ret}}\right)}{\left|\boldsymbol{r}^{\prime}-\boldsymbol{r}\right|}-\nabla_{r^{\prime}}\left(\frac{\rho\left(\boldsymbol{r}^{\prime}, t_{\mathrm{ret}}\right)}{\left|\boldsymbol{r}^{\prime}-\boldsymbol{r}\right|}\right)+\frac{\nabla_{\boldsymbol{r}^{\prime}} \rho\left(\boldsymbol{r}^{\prime}, t_{\mathrm{ret}}\right)}{\left|\boldsymbol{r}^{\prime}-\boldsymbol{r}\right|}\right] \\
& =\frac{1}{4 \pi \epsilon_{0}} \int d r^{\prime} \frac{\nabla_{r} \rho\left(\boldsymbol{r}^{\prime}, t_{\mathrm{ret}}\right)+\nabla_{r^{\prime}} \rho\left(\boldsymbol{r}^{\prime}, t_{\mathrm{ret}}\right)}{\left|\boldsymbol{r}^{\prime}-\boldsymbol{r}\right|} \\
& =\frac{1}{4 \pi \epsilon_{0}} \int \frac{d r^{\prime}}{\left|\boldsymbol{r}^{\prime}-\boldsymbol{r}\right|}\left[\frac{\partial \rho\left(\boldsymbol{r}^{\prime}, t_{\mathrm{ret}}\right)}{\partial t_{\mathrm{ret}}} \nabla_{r} t_{\mathrm{ret}}+\frac{\partial \rho\left(\boldsymbol{r}^{\prime}, t_{\mathrm{ret}}\right)}{\partial \boldsymbol{r}^{\prime}}+\frac{\partial \rho\left(\boldsymbol{r}^{\prime}, t_{\mathrm{ret}}\right)}{\partial t_{\mathrm{ret}}} \nabla_{r^{\prime}} t_{\mathrm{ret}}\right],
\end{aligned}
$$

where we used the requirement that $\rho$ must vanish at infinity in the second line, and expanded the operator $\nabla_{r^{\prime}}$ in terms of partial derivatives in the final line. Using $\nabla_{r} t_{\text {ret }}=-\nabla_{r^{\prime}} t_{\text {ret }}$, we obtain

$$
\nabla_{r} \phi=\frac{1}{4 \pi \epsilon_{0}} \int \frac{d r^{\prime}}{\left|\boldsymbol{r}^{\prime}-\boldsymbol{r}\right|} \frac{\partial \rho\left(\boldsymbol{r}^{\prime}, t_{\mathrm{ret}}\right)}{\partial \boldsymbol{r}^{\prime}}
$$

\subsubsection{Vector Potential}

For the vector potential we start from

$$
\boldsymbol{A}=\frac{\mu_{0}}{4 \pi} \int d r^{\prime} \boldsymbol{v}\left(\boldsymbol{r}^{\prime}, t_{\mathrm{ret}}\right) \frac{\rho\left(\boldsymbol{r}^{\prime}, t_{\mathrm{ret}}\right)}{\left|\boldsymbol{r}^{\prime}-\boldsymbol{r}\right|}
$$


to find

$$
\begin{aligned}
\frac{\partial \boldsymbol{A}}{\partial t} & =\frac{\mu_{0}}{4 \pi} \int \frac{d r^{\prime}}{\left|\boldsymbol{r}^{\prime}-\boldsymbol{r}\right|}\left[\frac{\partial \boldsymbol{v}\left(\boldsymbol{r}^{\prime}, t_{\mathrm{ret}}\right)}{\partial t} \rho\left(\boldsymbol{r}^{\prime}, t_{\mathrm{ret}}\right)+\boldsymbol{v}\left(\boldsymbol{r}^{\prime}, t_{\mathrm{ret}}\right) \frac{\partial \rho\left(\boldsymbol{r}^{\prime}, t_{\mathrm{ret}}\right)}{\partial t}\right] \\
& =\frac{\mu_{0}}{4 \pi} \int \frac{d r^{\prime}}{\left|\boldsymbol{r}^{\prime}-\boldsymbol{r}\right|}\left[\frac{\partial \boldsymbol{v}\left(\boldsymbol{r}^{\prime}, t_{\mathrm{ret}}\right)}{\partial t} \rho\left(\boldsymbol{r}^{\prime}, t_{\mathrm{ret}}\right)-\boldsymbol{v}\left(\boldsymbol{r}^{\prime}, t_{\mathrm{ret}}\right)\left(\nabla_{r^{\prime}} \cdot\left[\boldsymbol{v}\left(\boldsymbol{r}^{\prime}, t_{\mathrm{ret}}\right) \rho\left(\boldsymbol{r}^{\prime}, t_{\mathrm{ret}}\right)\right]\right)\right],
\end{aligned}
$$

where we have used the continuity equation $\partial \rho / \partial t=-\operatorname{div}(\rho \boldsymbol{v})$. We assume that the bunch length is much smaller than the scale over which the velocity $\boldsymbol{v}$ varies - the latter is associated with the distance over which the magnetic field changes, or the curvature of the trajectory. This allows us to neglect longitudinal derivatives of the velocity in comparison with the derivatives of $\rho$, i.e. $\operatorname{div}(\rho \boldsymbol{v}) \approx \boldsymbol{v} \cdot \nabla \rho$. We will also assume that $\boldsymbol{v}$ does not depend on time; all beam particles pass a given point, $\boldsymbol{r}$, with the same velocity regardless of their arrival time at $\boldsymbol{r}$. Hence $\partial \boldsymbol{v} / \partial t=0$, and Eq. (8.8) is

$$
\frac{\partial \boldsymbol{A}}{\partial t}=-\frac{1}{4 \pi \epsilon_{0} c^{2}} \int \frac{d r^{\prime}}{\left|\boldsymbol{r}^{\prime}-\boldsymbol{r}\right|} \boldsymbol{v}\left(\boldsymbol{r}^{\prime}\right)\left(\boldsymbol{v}\left(\boldsymbol{r}^{\prime}\right) \cdot \frac{\partial \rho\left(\boldsymbol{r}^{\prime}, t_{\mathrm{ret}}\right)}{\partial \boldsymbol{r}^{\prime}}\right)
$$

where we have used $\mu_{0} \equiv 1 / c^{2} \epsilon_{0}$.

Combining Eqs. (8.6) and (8.9) with Eq. (8.1) gives energy change

$$
\frac{d W}{d t}(\boldsymbol{r}, t)=\frac{q}{4 \pi \epsilon_{0}} \int \frac{d r^{\prime}}{\left|\boldsymbol{r}^{\prime}-\boldsymbol{r}\right|}\left[\frac{1}{c^{2}}\left(\boldsymbol{v}(\boldsymbol{r}) \cdot \boldsymbol{v}\left(\boldsymbol{r}^{\prime}\right)\right)\left(\boldsymbol{v}\left(\boldsymbol{r}^{\prime}\right) \cdot \frac{\partial \rho\left(\boldsymbol{r}^{\prime}, t_{\mathrm{ret}}\right)}{\partial \boldsymbol{r}^{\prime}}\right)-\boldsymbol{v}(\boldsymbol{r}) \cdot \frac{\partial \rho\left(\boldsymbol{r}^{\prime}, t_{\mathrm{ret}}\right)}{\partial \boldsymbol{r}^{\prime}}\right]
$$

Note that this equation includes effects of both space charge and radiation-the former results only in energy exchange between different parts of the beam, while the latter also causes energy loss.

For the case of straight motion with constant velocity, Eq. (8.10) reduces to

$$
\frac{d W}{d t}=\frac{q\left(1-\beta^{2}\right)}{4 \pi \epsilon_{0}} \int \frac{d r^{\prime}}{\left|\boldsymbol{r}^{\prime}-\boldsymbol{r}\right|}\left(\boldsymbol{v} \cdot \frac{\partial \rho\left(\boldsymbol{r}^{\prime}, t_{\mathrm{ret}}\right)}{\partial \boldsymbol{r}^{\prime}}\right) .
$$

To find the total change in energy, we then integrate $d W / d t$ over time along the particle's trajectory.

\subsubsection{Bunch Shape and Integration}

Integrating Eq. 8.10 numerically presents a challenge due to the apparent singularity at $\boldsymbol{r}=\boldsymbol{r}^{\prime}$. Physically, the singularity must disappear in the integration, so we can facilitate the numerical code by changing to polar coordinates, which cancels the singularity before we integrate. Defining $\theta \equiv 0$ in the positive $\hat{z}$ direction, we have transformation

$$
\begin{aligned}
& r \equiv\left|\boldsymbol{r}^{\prime}-\boldsymbol{r}\right| \\
& x^{\prime}-x=r \sin (\theta) \\
& z^{\prime}-z=r \cos (\theta)
\end{aligned}
$$

Substituting for $x^{\prime}$ and $z^{\prime}$ in the expression for $\rho$ and switching $d z^{\prime} d x^{\prime} \rightarrow r d r d \theta$, the integration factor $r$ cancels the singularity $\left|\boldsymbol{r}^{\prime}-\boldsymbol{r}\right|^{-1}$ in Eq.8.10.

Finally, we assume a Gaussian distribution, $\rho$, and without loss of generality normalize lengths to $\sigma_{z} \equiv 1$, giving

$$
\rho=\frac{1}{2 \pi \sigma_{z}} \exp \left[-\frac{\left(x^{\prime}-x_{c}\left(t_{\mathrm{ret}}\right)\right)^{2}}{2 \sigma_{x}^{2}}-\frac{\left(z^{\prime}-z_{c}\left(t_{\mathrm{ret}}\right)\right)^{2}}{2}\right] .
$$

Plugging into Eq.(8.10) above, we can then choose a specific bunch motion, $\boldsymbol{v}(r)$, and solve for $d W / d t$ numerically. 


\subsection{Comparisons}

\subsubsection{Straight Motion along $\mathrm{Z}$ Axis}

We bench-mark the code against several known analytical solutions, starting with the simplest case of a bi-gaussian bunch in $\hat{x}$ and $\hat{z}$, moving in the $\hat{z}$ direction. The potential for a stationary bunch is given by [91]

$$
\phi(x, z)=\sqrt{\frac{2}{\pi}} \frac{1}{\sigma_{x} \sigma_{z}} \int_{0}^{\infty} d \lambda \frac{e^{-\frac{x^{2} \lambda^{2}}{2 \lambda^{2} \sigma_{x}^{2}}-\frac{z^{2} \lambda^{2}}{2 \lambda^{2} \sigma_{z}^{2}}}}{\sqrt{\lambda^{2}+\sigma_{x}^{-2}} \sqrt{\lambda^{2}+\sigma_{z}^{-2}}} .
$$

Starting with reference points $x, z$ and bunch parameter $\sigma_{z}$ in the lab frame, we take $(x, z) \rightarrow(x, \gamma z)$ and $\left(\sigma_{x}, \sigma_{z}\right) \rightarrow\left(\sigma_{x}, \gamma \sigma_{z}\right)$ to calculate the potential in the rest frame. Then differentiating in the rest frame gives the fields $E_{z}^{\prime}, E_{x}^{\prime}$. We are interested only in the longitudinal field, so in the lab frame we simply have $E_{z}=E_{z}^{\prime}$. Comparison to our code is given in Fig.8.1.

\subsubsection{Straight Motion at an Angle}

In the previous section, we assumed that the motion of the bunch was along the $\hat{z}$ axis. More generally, we can benchmark the code to the case of a bi-gaussian bunch moving at a constant velocity at an arbitrary angle to the bunch. A bi-gaussian distribution can be described by an ellipse

$$
\frac{\tilde{x}^{2}}{2 \sigma_{\tilde{x}}^{2}}+\frac{\tilde{z}^{2}}{2 \sigma_{\tilde{z}}^{2}}-1=0
$$

with major and minor axes of $\sqrt{2} \sigma_{x}$ and $\sqrt{2} \sigma_{z}$. The bunch moves in the $\hat{z}$ direction, at an angle $\theta$ to the bunch $\tilde{z}$ axis. Rotating to the $\hat{z}, \hat{x}$ axes, and boosting $(z \rightarrow z / \gamma)$, we find the distribution in the rest frame

$$
\left(\sigma_{\tilde{x}}^{2} \cos (\theta)^{2}+\sigma_{\tilde{z}}^{2} \sin (\theta)^{2}\right) z^{2}+2\left(\sigma_{\tilde{x}}^{2}-\sigma_{\tilde{z}}^{2}\right) \cos (\theta) \sin (\theta) x z+\left(\sigma_{\tilde{z}}^{2} \cos (\theta)^{2}+\sigma_{\tilde{x}}^{2} \sin (\theta)^{2}\right) x^{2}-\sigma_{\tilde{z}}^{2} \sigma_{\tilde{x}}^{2} / 2=0
$$

From the general description of a quadratic form, $A z^{2}+2 B x z+C x^{2}+D x+F z+G=0$, we define

$$
\begin{aligned}
A & =\sigma_{\tilde{x}}^{2} \cos (\theta)^{2}+\sigma_{\tilde{z}}^{2} \sin (\theta)^{2} \\
B & =\left(\sigma_{\tilde{x}}^{2}-\sigma \tilde{z}^{2}\right) \cos (\theta) \sin (\theta) \\
C & =\sigma_{\tilde{z}}^{2} \cos (\theta)^{2}+\sigma_{\tilde{x}}^{2} \sin (\theta)^{2} \\
D & =0 \\
F & =0 \\
G & =-\sigma_{\tilde{z}}^{2} \sigma_{\tilde{x}}^{2} / 2
\end{aligned}
$$

giving the new angle of rotation between the rest frame ellipse axis, $\tilde{z}^{\prime}$, and the direction of motion, $\hat{z}$,

$$
\theta^{\prime}=\frac{1}{2} \cot ^{-1}\left(\frac{C-A}{2 B}\right)
$$

as well as the new rest frame major and minor axes

$$
\sigma_{\tilde{z}^{\prime}}^{\prime}=\sqrt{\frac{2\left(A F^{2}+C D^{2}+G B^{2}-2 B D F-A C G\right)}{\left(B^{2}-A C\right)\left((C-A) \sqrt{1+4 B^{2} /(A-C)^{2}}-(A+C)\right)}}
$$




$$
\sigma_{\tilde{x}^{\prime}}^{\prime}=\sqrt{\frac{2\left(A F^{2}+C D^{2}+G B^{2}-2 B D F-A C G\right)}{\left(B^{2}-A C\right)\left((A-C) \sqrt{1+4 B^{2} /(A-C)^{2}}-(A+C)\right)}} .
$$

We take simplest case of a reference point, $(z, 0)$, lying along the lab frame $\tilde{z}$ axis. Then in the rest frame, expressed in terms of the ellipse axes $\tilde{z}^{\prime}, \tilde{x}^{\prime}$, we have a new reference point $\left(z^{\prime}, x^{\prime}\right)$, with

$$
\begin{aligned}
& z^{\prime}=\cos \left(\theta^{\prime}-\alpha^{\prime}\right) z \sqrt{\gamma^{2} \cos (\theta)^{2}+\sin (\theta)^{2}} \\
& x^{\prime}=\sin \left(\theta^{\prime}-\alpha^{\prime}\right) z \sqrt{\gamma^{2} \cos (\theta)^{2}+\sin (\theta)^{2}}
\end{aligned}
$$

where

$$
\alpha^{\prime}=\tan ^{-1}(\tan (\theta) / \gamma)
$$

(It would be messy, but not difficult, to extend this to more general reference coordinates.)

Finally, with the new reference points $x^{\prime}, z^{\prime}$ and new distribution, $\sigma_{x}^{\prime}, \sigma_{z}^{\prime}$ given for the rest frame, we can use the analytical form, Eq.8.14, to calculate the rest frame potential, and then the rest frame fields $E_{x^{\prime}}^{\prime}$ and $E_{z^{\prime}}^{\prime}$. We are interested in the component along the direction of motion, $E_{v}=E_{\hat{z}}^{\prime}=\cos \left(\theta^{\prime}\right) E_{z^{\prime}}^{\prime}+\sin \left(\theta^{\prime}\right) E_{x^{\prime}}^{\prime}$. In Fig. 8.1, we've plotted the result from our code against this analytical result.

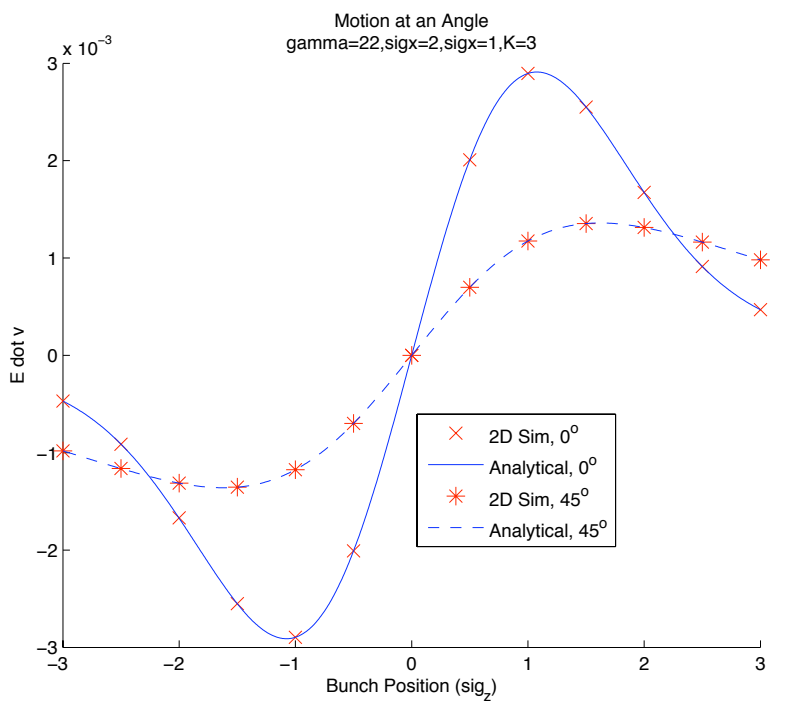

Figure 8.1: Comparison of code to analytical result for straight motion and motion at an angle. The dotted line shows the case, $\sigma_{x}=2, \sigma_{z}=1$ and $\theta=\pi / 4$.

\subsubsection{Coherent Synchrotron Radiation: Circular Motion}

As another benchmark, we check our code in the Coherent Synchrotron Radiation (CSR) limit of a pencil bunch $\left(\sigma_{z} \gg \sigma_{x}\right)$ moving in circular motion. Working in polar coordinates, with the radius measured from the center of the circle, and $\theta$ measured clockwise from the negative $z$ direction, we have

$$
v_{x}=\beta c \cos (\theta), v_{z}=\beta c \sin (\theta)
$$


with the bunch center at

$$
x_{c}(t)=R \cos \left(\frac{\beta c}{R} t\right), z_{c}(t)=R \sin \left(\frac{\beta c}{R} t\right), \theta_{c}=\arctan \left(-\frac{x_{c}}{z_{c}}\right)
$$

where $\theta_{c}$ is the angular position of the bunch at time, $t$. In the limit of a pencil beam following the circular path, we can rewrite the bunch profile as

$$
\rho(r, \theta)=\frac{1}{2 \pi \sigma_{x}} \exp \left[-\frac{(r-R)^{2}}{2 \sigma_{r}^{2}}-\frac{R^{2}\left(\theta^{\prime}-\theta_{c}\right)^{2}}{2 \sigma_{z}^{2}}\right]
$$

so that the bunch follows the circular path, and $\sigma_{x} \rightarrow \sigma_{r}$. Then, plugging into Eq.8.10, we can solve for $d W / d t$.

As a comparison, we use the well known solution $[92,93]$

$$
E_{\|}(z)=-\frac{2}{R^{2 / 3} 3^{1 / 3}} \int_{-\infty}^{z} d z^{\prime} \frac{1}{z^{\prime / 3}} \frac{\partial \lambda\left(z^{\prime}\right)}{\partial z^{\prime}}
$$

where $\lambda(z)$ is a 1-D Gaussian distribution $\left(\sigma_{r} \rightarrow 0\right)$. We compare this field to the circular case for our code, in the limit that $R \rightarrow \infty, \gamma \rightarrow \infty$, and $\sigma_{x} \rightarrow 0$ (Fig. 8.2).

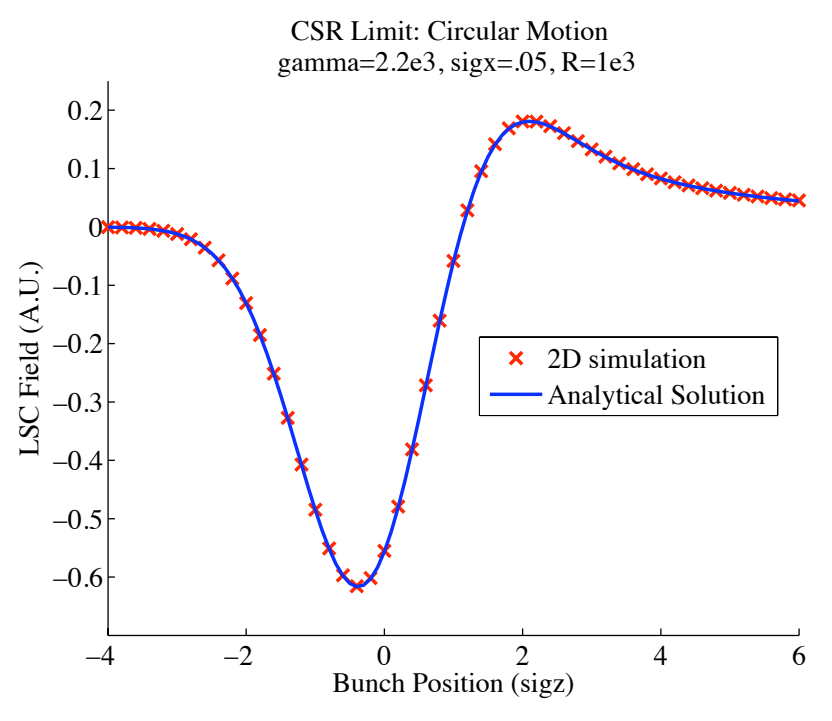

Figure 8.2: Benchmark for circular motion in the CSR limit. We compare our numerical code to the analytical solution (Eq. 8.26).

\subsubsection{Coherent Synchrotron Radiation: Undulator Motion}

We can also check the case of motion in a planar undulator in the CSR limit. The velocity, $\boldsymbol{v}$, is given by

$$
v_{x}=-\frac{K \beta c}{\gamma} \sin \left(k_{u} z\right), \quad v_{z} \approx \beta c\left(1-\frac{K^{2}}{4 \gamma^{2}}\right)+\frac{K^{2} \beta c}{4 \gamma^{2}} \cos \left(2 k_{u} z\right)
$$


where we've used $v_{z}=\sqrt{1-v_{x}^{2}}$, expanded in the limit of small transverse velocity and used trig identities to simplify $v_{z}$. We can then integrate to find the bunch center at time, $t$

$$
\begin{aligned}
& x_{c}(t)=\frac{K}{k_{u} \gamma} \cos \left(k_{u} \beta c\left(1-\frac{K^{2}}{4 \gamma^{2}}\right) t\right) \\
& z_{c}(t)=\beta c\left(1-\frac{K^{2}}{4 \gamma^{2}}\right) t+\frac{K^{2}}{8 k_{u} \gamma^{2}} \sin \left(2 k_{u} \beta c\left(1-\frac{K^{2}}{4 \gamma^{2}}\right) t\right) .
\end{aligned}
$$

In the integration step, we need to calculate the density at position $\left(x^{\prime}, z^{\prime}\right)$. We assume the beam snakes along the undulator path, so that the vertical $(x)$ beam center varies as a function of the $z$ position in the bunch. To calculate the E field, we need to find the charge density at the position of the source charge, $z^{\prime}$. In the $z$-direction, this distance is determined by the length of the undulator path, but for large $\gamma$ it is approximately equal to the longitudinal separation $\left(z^{\prime}-z_{c}\left(t_{r}\right)\right)$, with $z_{c}\left(t_{r}\right)$ the center of the bunch in retarded time. We then find the $z$-dependence of the density is approximately $\rho \propto \exp \left[\left(z^{\prime}-z_{c}\left(t_{r}\right)\right) / \sigma_{z}\right]$. For a fat beam, we can make the same approximation for the transverse position, $x$, but in the pencil beam limit we need to take $\rho \propto \exp \left[\left(x^{\prime}-x_{u}\left(z^{\prime}\right)\right) / \sigma_{x}\right]$, where $x_{u}\left(z^{\prime}\right)$ is the center of the undulator path at longitudinal position $z^{\prime}$.

For the pencil beam limit, a numerical integration over all space behaves poorly. Instead, we only integrate a thin strip along the undulator path, with the vertical thickness determined by $\sigma_{x}$.

For the analytical check, we use the expression in [94]

$$
\frac{d E}{d t}=k_{w} \int_{-\infty}^{s} d s^{\prime} G\left(\frac{\left(s-s^{\prime}\right) \gamma^{2} k_{w}}{K}\right) \frac{d \lambda\left(s^{\prime}\right)}{d s^{\prime}}
$$

with $\lambda(s)=1 / \sqrt{2 \pi} \exp \left[-s^{2} / 2 \sigma_{s}^{2}\right]$. To solve for $G(\zeta)$, we split our integration into 3 regimes. For $\zeta \sim 1$, we use Eq. 11 of [94]. In the asymptotic limits, we use

$$
G(\zeta)= \begin{cases}-0.99 \zeta^{-1 / 3} & \zeta \ll 1 \\ \frac{1}{2 \zeta}\left(\sum_{n=0}^{\infty}\left[J_{n}\left(\frac{2 n+1}{2}\right)-J_{n+1}\left(\frac{2 n+1}{2}\right)\right]^{2} \cos (4 \zeta(2 n+1))-1\right) & \zeta \gg 1\end{cases}
$$

Eq. 8.29 is derived by explicitly removing the zero-acceleration space-charge component [95]. While space charge falls off as $1 / \gamma^{2}$, the radiative component falls off more slowly, so to compare to our numerical code, we choose the limit of large $\gamma$. The comparison is given in Fig. 8.3.

\subsubsection{Space Charge Limit: Undulator Motion}

We now consider the space charge limit, $\sigma_{x}^{2} k_{u} \gg \sigma_{z}$ to suppress CSR, in a plane undulator. The velocity $\boldsymbol{v}$, and bunch positions at time $t$ are again given by Eqs. 8.27 and 8.28. After averaging over a full period, the undulator motion should match the case of straight motion with $\gamma \rightarrow \gamma_{z} \equiv \gamma / \sqrt{1+K^{2} / 2}$ [41]. We compare our code with energy $\gamma$ to Eq. 8.14 with energy $\gamma_{z}$ in Fig.8.4.

\section{Effect on ESASE}

Using $\gamma_{z}$ instead of $\gamma$ to calculate the longitudinal space charge effect, we can use a 1D simulation to check the effect on an LCLS eSASE-like simulation. Results are given in Fig.8.5. The saturation length is slightly longer, but the effect is minor. (The energy change from space charge counters the FEL-induced energy loss at the front of the bunch. Due to slippage, the front of a short electron bunch produces most of the FEL power, so the space charge induced electron energy loss at the rear has relatively little effect.) 


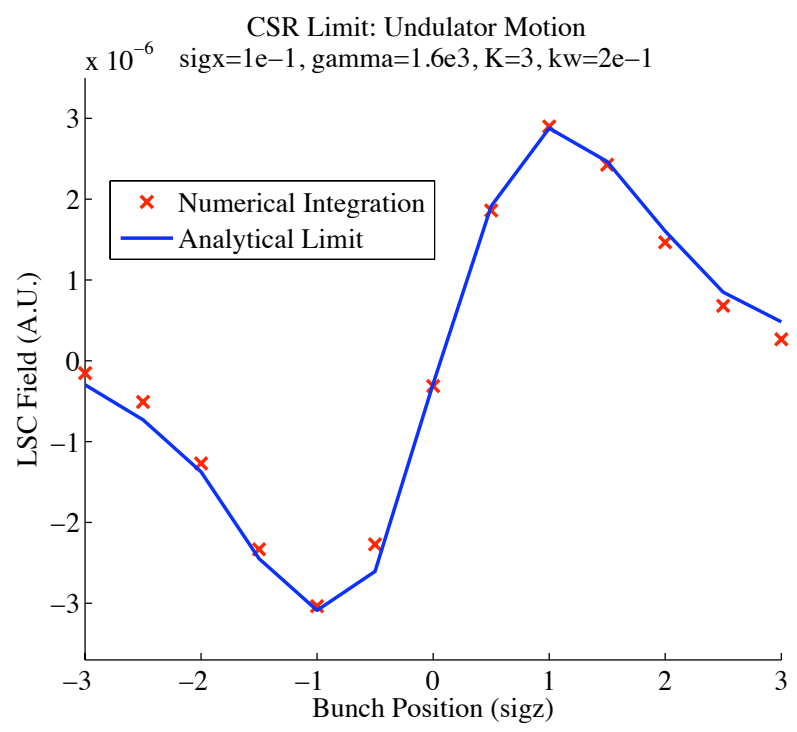

Figure 8.3: CSR check for undulator motion.

\begin{tabular}{|l|c|}
\hline Undulator period $\left(k_{w}\right)$ & .2 \\
Undulator parameter $(\mathrm{K})$ & 3 \\
Beam energy $(\gamma)$ & 13 \\
Transverse beam size $\left(\sigma_{x}\right)$ & 25 \\
\hline
\end{tabular}

Table 8.1: Parameters used for undulator space-charge simulation. All lengths normalized to units of longitudinal bunch length, $\sigma_{z}$.

It is possible to adjust the undulator taper, or equivalently use a phase shift (see chapter 9), to offset the energy change from space charge. In Fig. 8.6, we compensate for the space charge effect by shifting the phase to match the resonant condition at the front of the bunch. (This worsens the phase match at the rear of the bunch, but the FEL is relatively weak there in saturation.)

\subsection{Extra Calculations}

\subsubsection{Integration Lengths}

In practice, we do not need to integrate out to infinity in $r$, because the electron bunch is finite in length. However, in the relativistic regime, the bunch appears to stretch backwards, so the integration limits can be quite large in the negative direction. (That is, at retarded time, $t_{r e t}$, the bunch center is far from $r=0$.) Practically, it is important to calculate this distance and set the integration limits accordingly.

\section{Straight Drift}

We want to integrate out far enough that,

$$
z+r \cos \theta-z_{c}\left(t_{r e t}\right)=\Delta
$$




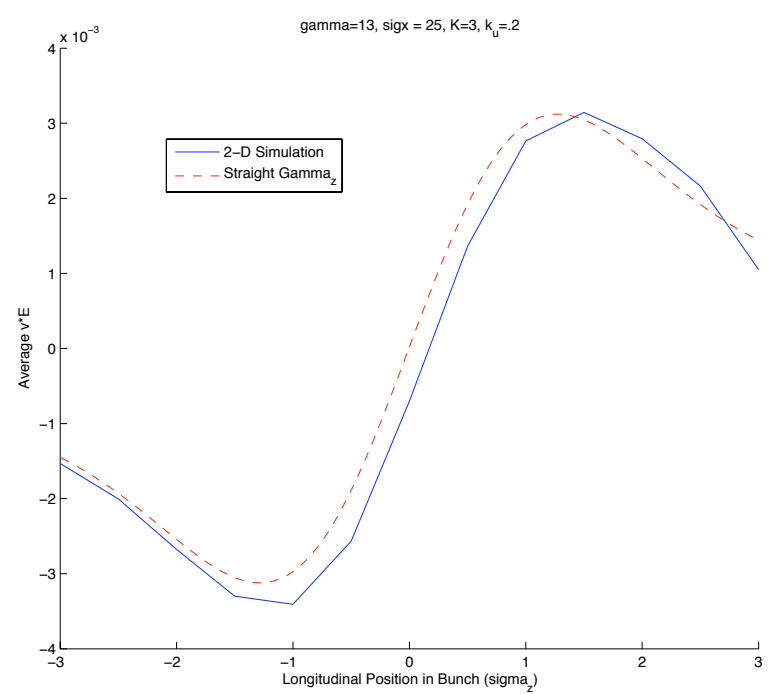

Figure 8.4: Comparison of undulator space charge at $\gamma$ to straight motion at $\gamma_{z}$.

where we arbitrarily choose $\Delta \sim 10 \sigma_{z}$. For straight motion, $z_{c}\left(t_{r e t}\right)=\beta c t_{r e t}, t_{r e t}=t-r / c$. If we take $t=0, z=0$, looking in the $\theta=\pi$ direction, we find an integration limit from $-(1-\beta) r_{\max }=\Delta$, or $r_{\max }=\frac{-\Delta}{1-\beta}$. For $\beta \rightarrow 1$, the numerical integral may fail for two reasons. First, the numerical integration is slow, since the space charge contribution decays as a slow function of $r$. Second, the density falls off at different rates depending on the angular direction (slowly for $\theta=\pi$, quickly for $\theta=0$ ). To avoid the second problem, we first integrate in $\theta$, then in $r$. (If we integrate first in $r$, with $r=0 \rightarrow 1 /(1-\beta)$, the quadrature looks many $\sigma_{z}$ out in the positive direction and decides the function is zero everywhere.)

We may also implement the code in Cartesian coordinates, being careful to step around the singularity at $r^{2}=x^{2}+z^{2}=0$. In this case, we can use the smaller integration limits

$$
\int_{-\infty}^{\infty} d z^{\prime} \int_{-\infty}^{\infty} d x^{\prime} \rightarrow \int_{-\frac{10 \sigma_{z}}{1-\beta}}^{10 \sigma_{z}} d z^{\prime} \int_{-10 \sigma_{x}}^{10 \sigma_{z}} d x
$$

\section{Undulator Motion}

For the undulator, we ignore the oscillation in $\hat{z}$ velocity in setting the integration limits (since we integrate over many undulator periods). The only change to the limits then, is that $v_{z}(t) \rightarrow \beta c\left(1-\frac{K^{2}}{4 \gamma^{2}}\right)$. So instead of velocity, $v=\beta c$, we have an average velocity $\bar{v}=\beta c\left(1-\frac{K^{2}}{4 \gamma^{2}}\right)$ and find

$$
r_{\max }=\frac{\Delta}{1-\beta\left(1-\frac{K^{2}}{4 \gamma^{2}}\right)}
$$

\section{Circular Motion}

For circular motion, the maximum distance depends on geometric factors, and is independent of $\beta$. Assuming the velocity is approximately equal to the speed of light, the particle falls behind the radiation because an arc length (particle path) is longer than a chord (radiation path). We are interested in the angle at which the 


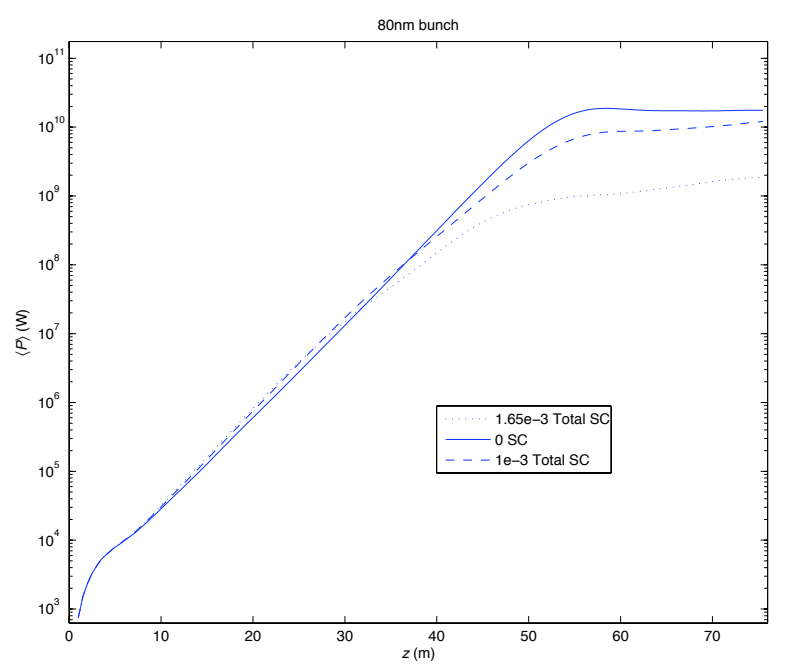

Figure 8.5: 1D FEL simulation for an 80nm bunch (LCLS parameters) when space charge produces a relative energy spread of $\sigma_{\delta} / \delta=10^{-3}$ at saturation (dashed line). The resulting power is slightly lower than the result without space charge $\gamma$ (solid line). When the space charge effect increases to $\sigma_{\delta} / \delta=1.65 \times 10^{-3}$ (dotted line), the power diminishes considerably.

particle falls a distance $\Delta$ behind the radiation. (e.g., when $\Delta=10 \sigma$, we can stop the integration.) The difference in path length, $\Delta=\rho \Psi$, is given by

$$
\begin{gathered}
\Delta=\rho \theta-2 \rho \sin \frac{\theta}{2} \\
\approx \rho\left(\theta-2\left(\frac{\theta}{2}-\frac{\theta^{3}}{48}\right)\right)=\rho \frac{\theta^{3}}{24} \\
\Rightarrow \theta \approx(24 \Psi)^{1 / 3}
\end{gathered}
$$

with $\Psi$ the angle over which the particle falls behind. (So the particle falls behind by $\Delta=10 \sigma_{z}$ when $\Psi=10 \sigma_{z} / \rho$, requiring integration out to $\theta=\left(\frac{240 \sigma_{z}}{\rho}\right)^{1 / 3}$.

\subsubsection{CSR Radiation}

We would like to use the code to evaluate the space charge effect in the regime where we can ignore coherent synchrotron radiation (CSR). The CSR radiation is only coherent if its wavelength, $\lambda$, is longer than the bunch length, $\sigma_{z}$. Otherwise radiation from different parts of the bunch will add incoherently and cancel. We then can ignore CSR when $\bar{\lambda}>\sigma_{z}$, with $\bar{\lambda} \equiv \lambda / 2 \pi$. For eSASE at LCLS, with $\sigma_{z} \approx 50 \mathrm{~nm}$, and $\lambda_{1}=1.5 \AA$, we have $\lambda \gg \lambda_{1}$. The off-axis undulator resonant wavelength is given by

$$
\lambda_{r}=\frac{\lambda_{u}}{2 \gamma^{2}}\left(1+\frac{K^{2}}{2}+\gamma^{2} \Psi^{2}\right)
$$

where $\Psi$ is the angle between the radiation and the $\mathrm{z}$ axis. For the FEL radiation, $\gamma \Psi<1$ and $\lambda_{1} \approx$ $\frac{\lambda_{u}}{2 \gamma^{2}}\left(1+\frac{K^{2}}{2}\right)$. However, for the CSR radiation, $\lambda_{C S R} \gg \lambda_{1}$, so $\gamma \Psi \gg K^{2}$, and we find, up to a factor of 2,

$$
\lambda_{C S R} \approx \lambda_{u} \Psi^{2}
$$




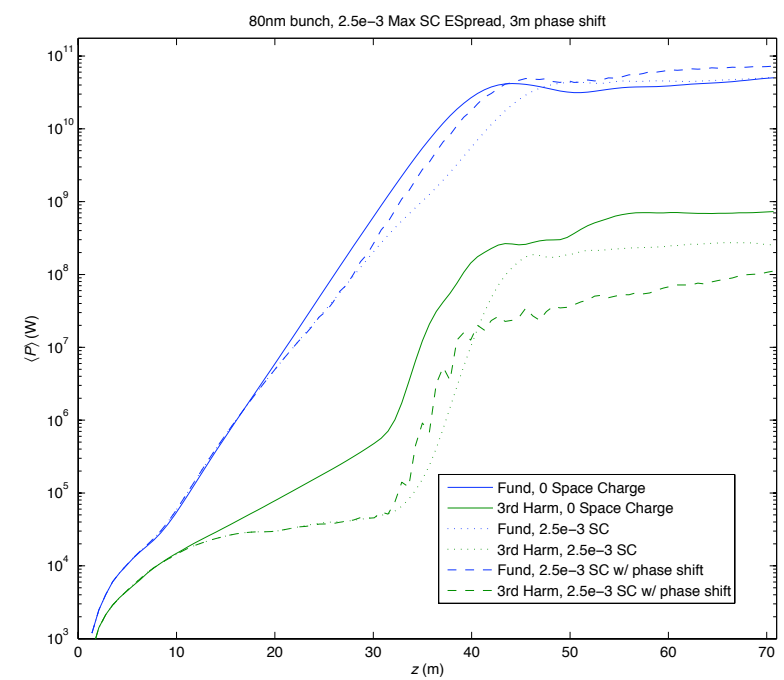

Figure 8.6: Phase shifts (dashed line) compensate for space charge effects and recover the resonant condition at the front of the bunch even with strong space charge $\left(\sigma_{\delta} / \delta=2.5 \times 10^{-3}\right.$ at saturation). The phase shifts (every $3 \mathrm{~m}$ ) are not frequent enough to keep the correct phase for the 3rd harmonic (green lines).

(Note, however, that $\lambda_{u} \approx 3 \mathrm{~cm}$, so $\lambda_{C S R} \ll \lambda_{u}$, and we still have $\Psi \ll 1$.)

The radiation must be coherent transversely as well. Looking from an angle $\Psi$, the radiation from the center to a point on the bunch edge, at $x=\sigma_{x}$, the phases will differ by roughly $\Psi \sigma_{x}$. For the radiation to be coherent, we must have $\Psi \sigma_{x}<\lambda_{C S R}$ so that the phase difference is insignificant relative to $\lambda_{C S R}$. Plugging in for $\Psi$ using Eq.8.33, we find CSR radiation only when, $\sigma_{x}<\sqrt{\lambda_{C S R} \lambda_{u}}$, or, conversely, the radiation is suppressed when $\sigma_{x}>\sqrt{\lambda_{C S R} \lambda_{u}}$. The dominant CSR radiation is on the order of $\sigma_{z}$, so we find the condition

$$
\sigma_{x}^{2} k_{u} \gg \sigma_{z}
$$




\section{Chapter 9}

\section{Enhancing FEL Power with Phase Shifters}

\subsection{Introduction}

Photon-hungry single molecule imaging XFEL applications may require more power than is available even from a saturated LCLS beam [42]. Exponential power growth is no longer possible once the FEL reaches saturation (when the bunching factor is maximal), but the total FEL pulse energy may still increase linearly along the undulator; the electrons simply continue to radiate as long as the microbunched electrons remain at the optimal phase relative to the radiation. In this section we consider optimizing this linear growth through the use of phase shifters.

A common method for increasing power past saturation is called 'tapering.' As the FEL removes energy from the electron beam, the resonant wavelength shifts away from the microbunching wavelength, stopping energy growth. Physically, the electron slippage per undulator period defines the resonant wavelength. (At this wavelength, the electrons radiate coherently with themselves.) As the electrons lose energy, they slip farther each period, and the resonant wavelength grows longer. By 'tapering' (decreasing the strength) of an undulator, the weaker magnet strengths offset the loss of electron energy, and the slippage remains constant.

We may also view the excess slippage as a phase error; each period the electrons slip slightly more than $2 \pi$ when the electron energy drops, and the result is an accumulated phase error. We can then view the undulator taper as an offsetting phase shift. Alternatively, we can simply insert electron phase shifters (e.g. a chicane to delay the electrons), which periodically restore the proper phase relation between the electron bunching and the radiation.

Varfolomeev et al. first described the use of phase shifts to enhance power in 1998 [43]. (We learned of this work following our study.) Past work has also considered the use of phase shifters to suppress power in the fundamental wavelength, so as to allow continued growth of the harmonics [96]. In the following sections, we undertake a detailed analytical and numerical study of enhancing FEL power with phase shifters. We explore the relation between phase shifts and undulator tapers to calculate optimal phase shifts for SASE FELs in the saturation regime and apply these shifts to simulations with LCLS-like parameters. The phase shift method, while equivalent to tapering the undulator parameter, provides an independent knob to maximize the FEL 
performance. Conversely, we may consider the use of an undulator as phase shift, as demonstrated in gain length studies with an undulator removed (chapter 4). The following results were first presented as Ref. [97].

\subsection{One-Dimensional Analysis}

The resonant condition

$$
\lambda_{r}=\lambda_{u} \frac{1+K^{2} / 2}{2 \gamma_{0}^{2}},
$$

sets the radiation wavelength, $\lambda_{r}$ for an FEL; after each undulator period, $\lambda_{u}$, the electron bunch slips behind the radiation by exactly $\lambda_{r}$. It follows that when the resonant condition holds, the phase, $\Psi$, between the electron bunch and radiation stays constant (up to $2 \pi$ ). Near saturation, the bunch loses significant energy to the FEL radiation, and the resonant condition begins to fail. As $\gamma_{0} \rightarrow \gamma<\gamma_{0}$, the resonant wavelength increases, and the electrons slip more than one radiation wavelength during each undulator period. Introducing a phase shift (by means of a small chicane) can correct for the increase in slippage by shifting the bunch backwards into the previous bucket. (That is, if the electrons accumulate extra slippage of $\Delta \theta$, then the chicane shifts the electrons an additional $2 \pi-\Delta \theta$. There is no easy way to shift electrons forward.) After the shift, the electron bunch is once again in phase with the radiation, preserving the resonant condition farther into saturation.

To solve for the optimal phase shift, we use a simplified 1-D FEL model. The slowly varying radiation field $\tilde{a}$ is given by

$$
\frac{d \tilde{a}}{d \bar{z}}=-\left\langle e^{-i \theta_{j}}\right\rangle
$$

The phases, $\theta \equiv\left(k+k_{u}\right) z-\omega t+$ const, are the longitudinal positions of the electrons relative to the electron bunch given in units of $\lambda_{r} / 2 \pi=1 / k$. The variable $\bar{z} \equiv 2 k_{u} \rho z$, is the scaled position along the undulator. Here $\rho$ is the dimensionless FEL parameter [11]. Finally, the average in Eq. (9.2) is taken over all electrons. Taking $\tilde{a} \equiv A e^{i \Psi}$, with $\Psi$ the phase of the radiation relative to the electron bunch, we can separate out the magnitude and phase components of the radiation field:

$$
\frac{d \tilde{a}}{d \bar{z}}=e^{i \Psi}\left[\frac{d A}{d \bar{z}}+i A \frac{d \Psi}{d \bar{z}}\right] .
$$

Inserting Eq. (9.3) into Eq. (9.2) and separating real and imaginary parts gives

$$
\begin{aligned}
& \frac{d A}{d \bar{z}}=-\left\langle\cos \left(\theta_{j}+\Psi\right)\right\rangle \\
& \frac{d \Psi}{d \bar{z}}=\frac{1}{A}\left\langle\sin \left(\theta_{j}+\Psi\right)\right\rangle .
\end{aligned}
$$

Our strategy is to introduce an arbitrary phase shift, $\phi$ between the radiation and electrons, and then maximize $\frac{d A}{d \bar{z}}$ with respect to $\phi$ at all points along the undulator. Our goal, then, is to choose $\phi$ to maximize the quantity $-\left\langle\cos \left(\theta_{j}+\Psi+\phi\right)\right\rangle$.

Adding the phase shift, $\phi$, gives

$$
\frac{d A}{d \bar{z}}=\left\langle\cos \left(\theta_{j}+\Psi\right)\right\rangle \cos (\phi)-\left\langle\sin \left(\theta_{j}+\Psi\right)\right\rangle \sin (\phi) .
$$

To find the optimal phase shift, we differentiate with respect to $\phi$ to find

$$
\phi_{\max }(\bar{z})=\arctan \left[-\frac{\left\langle\sin \left(\theta_{j}+\Psi\right)\right\rangle}{\left\langle\cos \left(\theta_{j}+\Psi\right)\right\rangle}\right] .
$$


Carrying out the maximization then requires the calculation of $\phi_{\max }(\bar{z})$ at all points past saturation along the undulator. (The phase shift $\phi$ is a function of $\bar{z}$, because $\theta_{j}$ and $\Psi$ are also functions of $\bar{z}$.)

We note that $\phi_{\max }<0$, which indicates that the electrons must move forward relative to the radiation. Because chicanes can only shift the electrons backwards, in practice we must move the electrons by a phase shift of $2 \pi+\phi_{\max }>0$.

Substituting the result from Eq. (9.7) into Eq. (9.6) we find, for $\left\langle\cos \left(\theta_{j}+\Psi\right)\right\rangle>0$,

$$
\left(\frac{d A}{d \bar{z}}\right)_{\max }=\sqrt{\left\langle\cos \left(\theta_{j}+\Psi\right)\right\rangle^{2}+\left\langle\sin \left(\theta_{j}+\Psi\right)\right\rangle^{2}} \equiv|b|
$$

where $b$ is the bunching factor of the electrons evaluated at the resonant wavelength.

We originally motivated the phase shift by the need to maintain a constant $\Psi$, the phase between the electron bunch and the radiation. We would like to check that our best phase, $\phi_{\max }$, optimized to increase the radiation amplitude, $A$, will simultaneously keep $\Psi$ fixed. Returning to Eq. (9.5), now with an arbitrary phase inserted, we have

$$
\frac{d \Psi}{d \bar{z}} \propto\left\langle\sin \left(\theta_{j}+\Psi\right)\right\rangle \cos \phi+\left\langle\cos \left(\theta_{j}+\Psi\right)\right\rangle \sin \phi .
$$

Plugging in $\phi_{\max }$ from the optimization condition, Eq. (9.7), gives

$$
\begin{aligned}
& \frac{d \Psi}{d \bar{z}} \propto\left\langle\sin \left(\theta_{j}+\Psi\right)\right\rangle-\left\langle\cos \left(\theta_{j}+\Psi\right)\right\rangle \frac{\left\langle\sin \left(\theta_{j}+\Psi\right)\right\rangle}{\left\langle\cos \left(\theta_{j}+\Psi\right)\right\rangle} \\
& =0 .
\end{aligned}
$$

We find that implementing the phase shifts, $\phi_{\max }$, does indeed keep the phase, $\Psi$, fixed, consistent with our initial goal of maintaining the correct slippage length.

\subsection{Equivalence to Undulator Taper}

To find the optimal phases, Eq. (9.7), we can numerically evaluate $\left\langle\sin \left(\theta_{j}+\Psi\right)\right\rangle$ and $\left\langle\cos \left(\theta_{j}+\Psi\right)\right\rangle$ at each desired phase shifter location. This, of course, is not very practical for an actual SASE FEL. However, we motivated this approach by the need to maintain the resonant condition (Eq.9.1). Tapering the undulator, an established method for optimizing FELs, works through the same principle [60]. From the resonant condition, we see that shifting the phase is equivalent to decreasing the undulator parameter. To express a phase shift in terms of a taper, we start with the ponderomotive phase equation

$$
\frac{d \theta}{d \bar{z}}=k_{u}-k \frac{\left[1+K^{2} / 2\right]}{2 \gamma^{2}}
$$

relating the change in phase, $\phi=\Delta \theta$, to the undulator parameter, $K$. An undulator taper is a small shift $K_{0} \rightarrow K(\bar{z})$. Plugging into Eq. (9.11), dropping the second order term, and using the resonant condition, $k_{u} / k=\left(1+K_{0}^{2} / 2\right) /\left(2 \gamma_{r}^{2}\right)$ gives

$$
\frac{d \theta}{d \bar{z}}=k_{u}-k_{u} \frac{\gamma_{r}^{2}}{\gamma^{2}}+k_{u} \frac{\gamma_{r}^{2}}{\gamma^{2}} \frac{K_{0}\left(K(\bar{z})-K_{0}\right)}{1+K_{0}^{2} / 2}
$$

Using the notation $\eta=\left(\gamma-\gamma_{r}\right) / \gamma_{r},|\eta| \ll 1$ yields the usual FEL phase equation with a correction term of order $K_{0}-K(\bar{z})$ :

$$
\frac{d \theta}{d \bar{z}}=2 k_{u}\left[\eta-\frac{K_{0}\left(K_{0}-K(\bar{z})\right)}{2+K_{0}^{2}}\right] .
$$


Thus we find a change, $K_{0} \rightarrow K(\bar{z})$, results in a change, $\theta \rightarrow \theta+\Delta \theta$, with accumulated phase shift

$$
\Delta \theta(\bar{z})=-2 k_{u} \int_{\bar{z}_{0}}^{\bar{z}} \frac{K_{0}\left(K_{0}-K\left(\bar{z}^{\prime}\right)\right)}{2+K_{0}^{2}} d \bar{z}^{\prime},
$$

where $K(\bar{z})$ is some arbitrary function (i.e. an optimized taper) and the integral starts from the last phase shift at position $\bar{z}_{0}$.

The advantage of deriving phase shifts in this manner is that SASE FEL undulator tapers are well understood, and the optimal function $K(\bar{z})$ can be calculated from the FEL parameters, for example by the GINGER self-taper algorithm [61]. With a predetermined taper function, we can plug into Eq. (9.14) and calculate the optimal phase shifts for an arbitrary FEL.

We have shown that shifting the phase continuously is identical to tapering the undulator parameter. However, a practical phase would be implemented discretely, with the taper from each undulator section replaced by a single shift. In this case, the exact equivalence breaks down. The larger the shift, the more time the FEL spends at a suboptimal phase, and for long undulators the accumulated difference $K\left(\bar{z}^{\prime}\right)-K_{0}$ may significantly degrade the FEL power (Eq. 9.14). To determine the practicality of discrete shifts, we use FEL parameters similar to the LCLS at $1.5 \AA$ [98] for numerical examples (Table 9.1). Although LCLS does not currently use periodic phase shifters, designs for similar projects such as the European XFELs [99] have incorporated phase shifters between undulator sections. Using Eq. (9.14), we find the taper-equivalent shifts for LCLS have $\Delta \theta<1$, suggesting that the method of phase shifts will match the performance of an undulator taper.

Table 9.1: SASE Simulation Parameters

\begin{tabular}{l|c|}
\hline Radiation wavelength $\left(\lambda_{r}\right)$ & $1.5 \AA$ \\
Bunch current & $3.4 \mathrm{kA}$ \\
Undulator period $\left(\lambda_{u}\right)$ & $3 \mathrm{~cm}$ \\
Undulator parameter $(\mathrm{K})$ & 3.5 \\
Electron energy & $13.6 \mathrm{GeV}$ \\
Relative RMS energy spread & $1 \times 10^{-4}$ \\
Normalized transverse emittance & $1.2 \mu \mathrm{m}$ \\
Beta function & $25 \mathrm{~m}$ \\
\hline
\end{tabular}

\subsection{Numerical Results}

\subsubsection{1-D Simulations}

To simulate phase shifts, we started with a 1-D FEL code, including energy spread but not emittance effects. We used four different types of optimization:

1. Phase scan,

2. Numerically optimized phase shifts,

3. Linear energy taper, and 
4. Phase shifts derived from a linear taper.

First we used a brute force phase scan. To determine the $n^{\text {th }}$ phase shift at position $z_{n}$, we optimized the power at position $z_{n+1}$ by trying 5 (or more) different phase shifts. Then, picking the best phase for the $n^{\text {th }}$ position, we optimized position $z_{n+1}$.

Second, we used the results from Eq. (9.7) to calculate optimal phase shifts numerically. We calculate the phase for 2 cases: shifts every $1 \mathrm{~m}$ and every $3 \mathrm{~m}$. The $3 \mathrm{~m}$ shifts are less effective than the phase scan because the shifts are infrequent, and $\Delta \theta>1$. The results for the first two methods are given in Fig. 9.1.

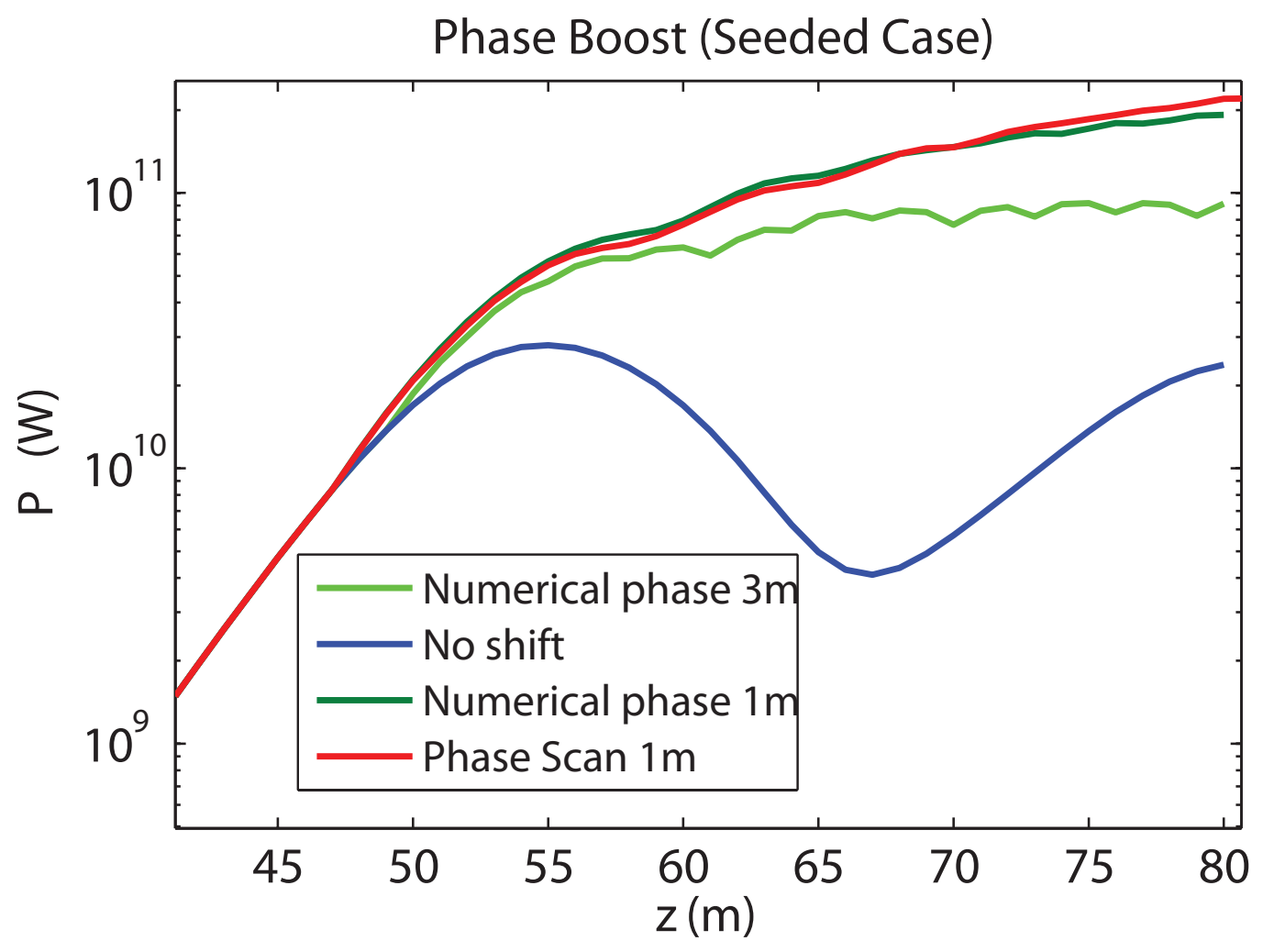

Figure 9.1: Fundamental radiation power from phase shifts. Phases done 3 ways: numerical optimization every $1 \mathrm{~m}$ (dark green), numerical optimization every $3 \mathrm{~m}$ (light green), phase scan every $1 \mathrm{~m}$ (red). The fourth line (blue) has no phase shifts.

Third, we used a linear taper to boost the fundamental power. Fourth, we calculated the taper-equivalent phase shifts (Eq. (9.14)), implemented every $1 \mathrm{~m}$. The final two methods are computationally identical if the phase is shifted on each iteration. However, the results still match for less frequent phase shifts, so long as the shifts are small $(\Delta \theta<1)$. Results for both seeded and SASE cases are given in Fig. 9.2. Performance is worse for the third harmonic (green), because the shifts are relatively larger than in the case of the fundamental.

Finally, we compare phase shifts derived from the optimal linear taper to numerically optimized phase shifts (Eq. (9.7)), and the results from the phase scan. The phase shifts are all well matched (Fig. 9.3), confirming the equivalence of the four optimization methods. 


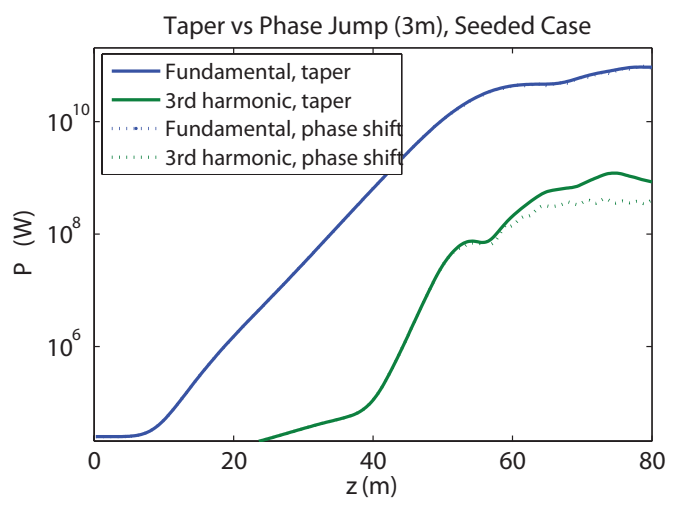

(a)

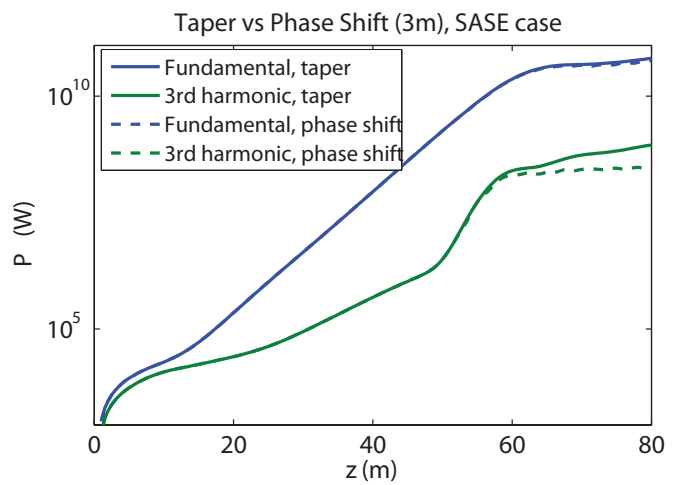

(b)

Figure 9.2: Comparison of taper and equivalent phase shifts every $3 \mathrm{~m}$ in 1D simulations for a Seeded FEL (left) and SASE FEL (right). In both cases, the phase shift is less effective for the third harmonic, due to the relatively larger phase errors.

\subsubsection{3-D Simulations}

We repeated the same study using the FEL code GINGER. To find an optimal undulator taper, $\Delta K(z)$, we used GINGER's self-taper algorithm [61]. With $\eta$ defined above

$$
\frac{d \eta}{d z}=-\frac{1}{\gamma_{0}} \frac{d \gamma_{r}}{d z}-\frac{e K[J J]}{2 \gamma_{0}^{2} m c_{2}} E \sin (\theta+\Psi)
$$

When the energy change is small (in the exponential regime), we can ignore the $\frac{d \gamma_{r}}{d z}$ term. However, at saturation, the energy loss is significant (of order $\rho$ ), and the resonant energy $\gamma_{r}$ changes. Following [60], we define a synchronous phase $\Psi_{r}$ from

$$
-\frac{1}{\gamma_{0}} \frac{d \gamma_{r}}{d z} \equiv \frac{e K[J J]}{2 \gamma_{0}^{2} m c_{2}} E \sin \left(\Psi_{r}\right)
$$

with $\Psi_{r}$ determined by both $\frac{d \gamma_{r}}{d z}$ and $K$. Any particle with the synchronous phase will then define the center of the bucket, with particles nearby in phase space performing a synchrotron oscillation around the synchronous phase. (By definition, the energy $\eta$ is constant.) An optimal phase $\Psi_{r}$ is determined by optimizing bucket size and $d \gamma_{r} / d z$, which together set the energy transferred from electrons to radiation. We can then find a function $K(z)$ to maintain the phase $\Psi_{r}$ as a constant throughout the saturation regime. Alternatively, we can manipulate the phase directly through periodic phase jumps, as described in Eq. (9.14) above. If the phase jumps are frequent enough so that $\Delta \theta<1$ (or equivalently, $\Delta \Psi$ ), the FEL will effectively maintain the resonant condition without resorting to altering $\mathrm{K}$.

Using GINGER's self-design taper function we create an optimal taper and then find the equivalent phase shifts (Fig. 9.4). We confirm the 1-D results, showing an equivalence between a taper and phase shift for an LCLS-like SASE FEL (Fig. 9.5, Table 9.1). The equivalence starts to fail at the very end of the undulator, when $\Delta \theta>1$. 


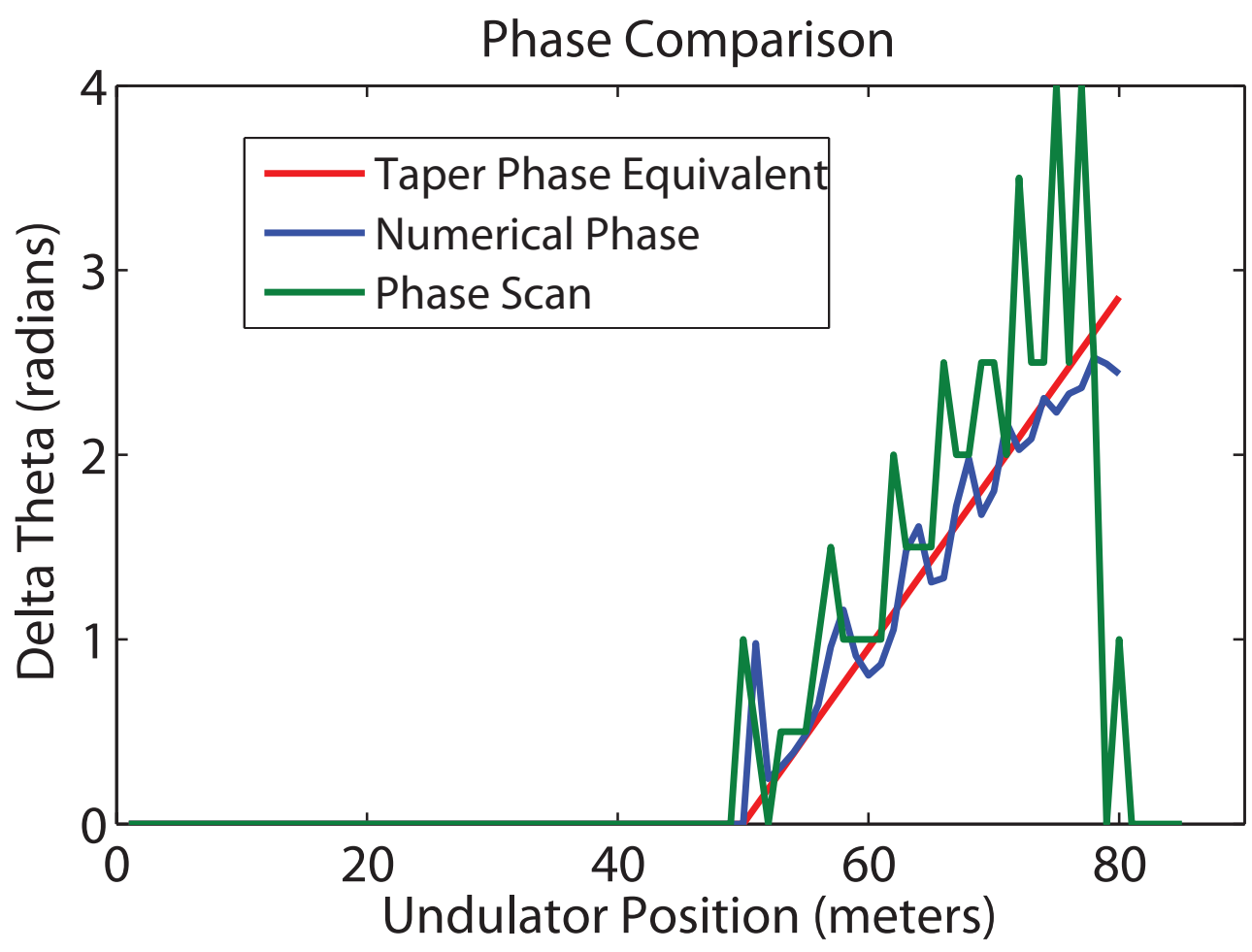

Figure 9.3: Phases determined by phase scan, numerical optimization and taper equivalent (Seeded case).

\subsection{Conclusion}

We demonstrate the equivalence between phase shifts and undulator tapers. If the required phase shifts are small $(\Delta \theta<1)$, then the shifts can be implemented discretely and still mimic the effect of an undulator taper. In particular, we simulate phase shifts placed between undulator sections for an x-ray FEL and find the results are equivalent to those of an optimized taper. The phase shift method could be useful as a replacement or enhancement of undulator tapers when the use of tapers is constrained by technical issues. Tapering undulators may also degrade the beam brightness by increasing the radiation bandwidth. Even when the undulator parameter of each undulator section can be individually adjusted, placing phase shifters between the undulator sections provides independent control and fine tuning capability over the FEL power as well as possibly improved brightness.

\subsection{Acknowledgments}

We would like to thank W. Fawley, B. McNeil, H.-D. Nuhn and J. Wu for many helpful discussions. 


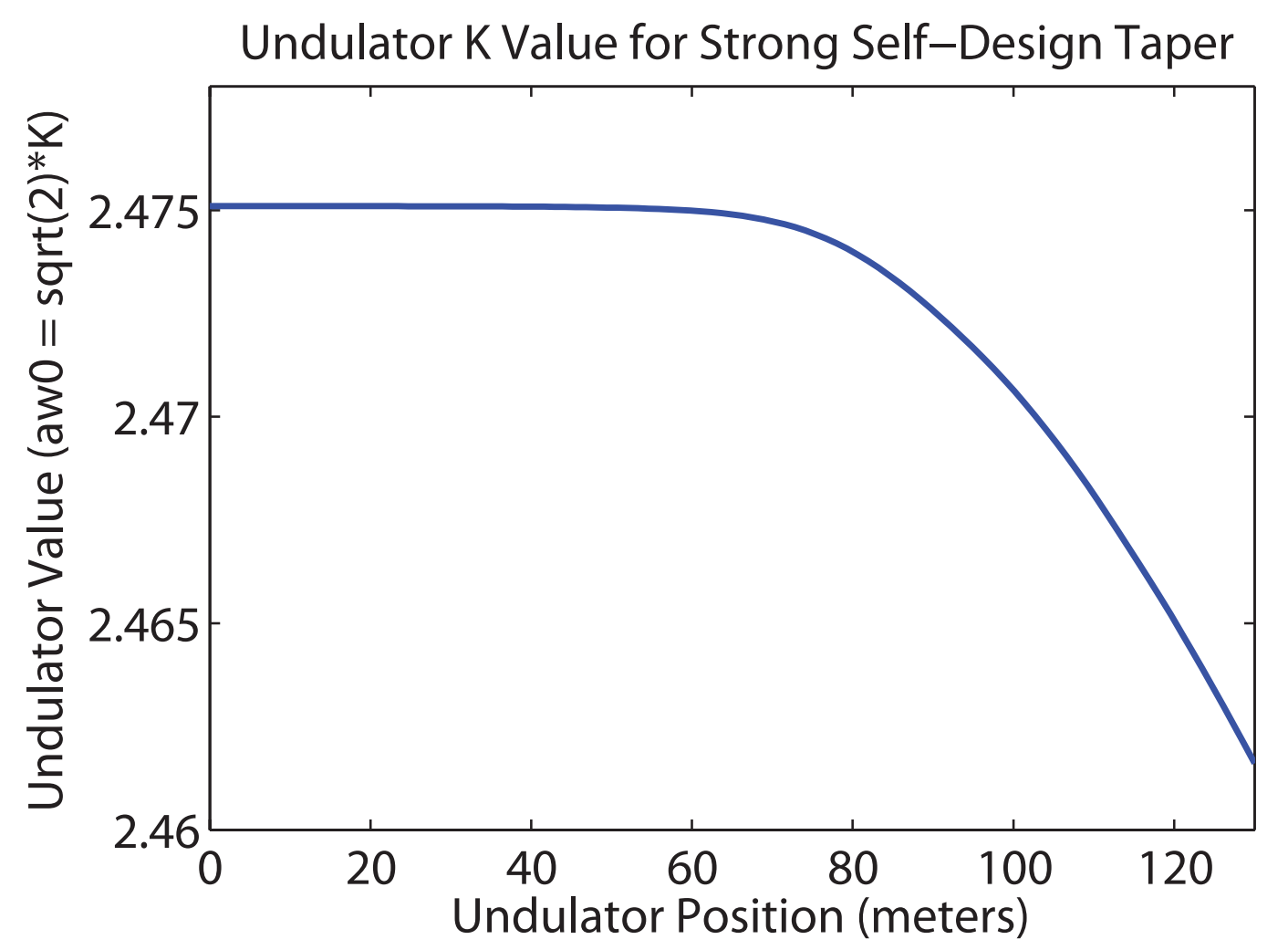

Figure 9.4: Taper for Seeded and SASE cases. Simulation using GINGER's 3D code. 


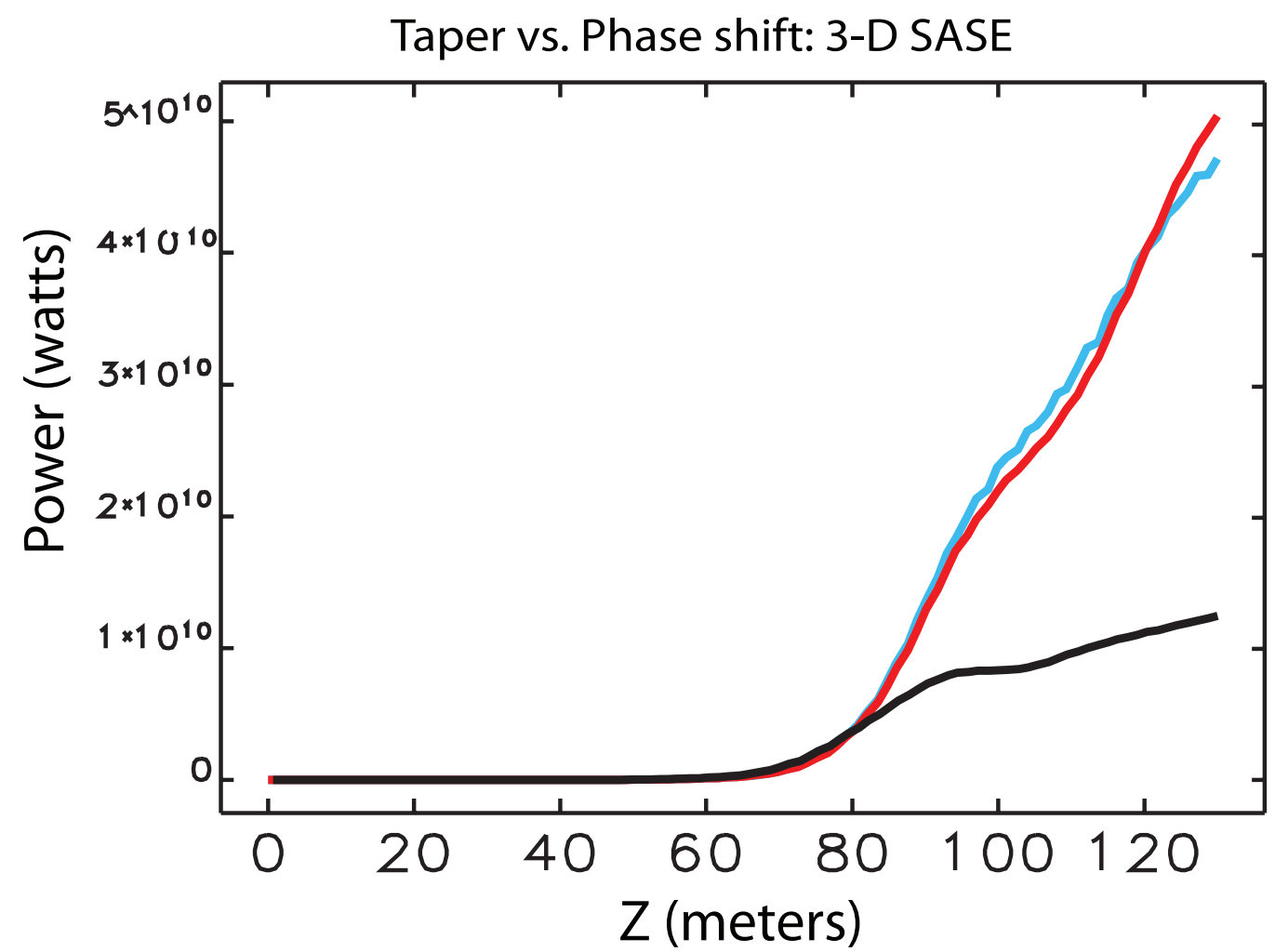

Figure 9.5: Power for normal (black), tapered (red) and phase-shifted (blue) LCLS-like SASE FEL, 3-D simulation. GINGER simulation uses 12 phase shifts between undulator sections (every $3.8 \mathrm{~m}$ ). 


\section{Appendix A}

\section{Comparison of 1D and 3D Models of Longitudinal Space Charge}

\section{A.1 Summary of Results}

Chapters 2 and 3 both focus on the effects of longitudinal space charge fields. In this appendix, we follow on the work of Venturini [25] to consider the differences between 1D and 3D models of space charge. The $3 \mathrm{D}$ results closely parallel the results of chapter 3 , though in the following section we consider flat-top, not Gaussian, transverse distributions. We also see that the 3D and 1D models are quite similar in the relevant limits for chapter 2.

Our goal, following [25], is to calculate the quantity $\left\langle E_{k}\left(r_{1}\right) E_{k}^{*}\left(r_{2}\right)\right\rangle$. This expression is closely related to the bunching factor, $b(k)$ from chapter 3 and the noise factor, $F(k)$, from chapter 2 . First, we consider the $1 \mathrm{D}$ case, calculated in full in [25]. Evaluating at the center of the beam $\left(r_{1}=r_{2}=0\right)$, Venturini finds

$$
\left\langle\tilde{E}_{k} \tilde{E}_{k}^{*}\right\rangle_{1 D: r_{1}, r_{2}=0}=\left(\frac{e}{2 \pi^{2} \epsilon_{0} \gamma r_{b}}\right)^{2} N_{b}\left(\frac{1-\xi_{b} K_{1}\left(\xi_{b}\right)}{\xi_{b}}\right)^{2} .
$$

(See Section A.2.1 for derivation.) However, for the noise factor, $F(k)$, we are interested in average over all particles in the beam, so we need the transverse average of $\left\langle E_{k}\left(r_{1}\right) E_{k}\left(r_{2}\right)^{*}\right\rangle$. If we assume a flat-top transverse distribution (a disc of charge with particles distributed randomly) of radius $r_{b}$, we can integrate to find

$$
\left\langle\tilde{E}_{k} \tilde{E}_{k}^{*}\right\rangle_{1 D \perp}=\left(\frac{e}{2 \pi^{2} \epsilon_{0} \gamma r_{b}}\right)^{2} N_{b}\left(\frac{1-2 K_{1}\left(\xi_{b}\right) I_{1}\left(\xi_{b}\right)}{\xi_{b}}\right)^{2}
$$

equivalent to the expression given in Eq. 2.24. (See Section A.3.1 for derivation.)

We now would like to compare Eqs. A.1 and A.2 to the results for a 3D analysis. Evaluating at $r=0$, Venturini finds (Eq.11 of [25])

$$
\left\langle\tilde{E}_{k} \tilde{E}_{k}^{*}\right\rangle_{3 D: r_{1}, r_{2}=0}=\left(\frac{e}{2 \pi^{2} \epsilon_{0} \gamma r_{b}}\right)^{2} N_{b} \frac{1+\xi_{b}^{2}\left[K_{0}^{2}\left(\xi_{b}\right)-K_{1}^{2}\left(\xi_{b}\right)\right]}{4} .
$$

(See Section A.2.2 for derivation.) Once gain, we are interested in the transverse average, so we repeat Venturini's calculation but this time integrate over the beam's transverse extent. Assuming the charge is 
distributed randomly across a disc of radius $r_{b}$, we find

$$
\left\langle\tilde{E}_{k} \tilde{E}_{k}^{*}\right\rangle_{3 D \perp}=\left(\frac{e}{2 \pi^{2} \epsilon_{0} \gamma r_{b}}\right)^{2} N_{b}\left[\left(\frac{1-2 K_{1}\left(\xi_{b}\right) I_{1}\left(\xi_{b}\right)}{\xi_{b}^{2}}\right)^{2}+\delta\right]
$$

where we have defined

$$
\delta(x) \equiv K_{1}\left(\xi_{b}\right)^{2}\left(\xi_{b} I_{1}\left(\xi_{b}\right) I_{2}\left(\xi_{b}\right)+\frac{\xi_{b}^{2}}{4}\left(I_{2}\left(\xi_{b}\right)^{2}-I_{1}\left(\xi_{b}\right)^{2}\right)\right)
$$

to rewrite the 3D result in the same form as the 1D result (Eq. A.2). (Derivation is given in Section A.3.3.) Comparing Eqs. A.2 and A.4, we see that the 1D and 3D expressions are quite similar; the only difference is the $\delta$ term, which is generally small.

To better understand the comparison with the $r=0$ results, we also formulate a numerical integral that we can average for an arbitrary transverse portion of the beam. For this $3 \mathrm{D}$ result we follow the same method as for the 1D calculation (as an additional check). We then numerically integrate over an arbitrary portion of the beam. So, for example, to integrate from 0 to some radius $r$, we evaluate

$$
\begin{aligned}
\left\langle\left\langle\tilde{E}_{k} \tilde{E}_{k}^{*}\right\rangle\right\rangle_{3 D \perp 0 \rightarrow r} & =\left(\frac{e}{2 \pi^{2} \epsilon_{0} \gamma r_{b}}\right)^{2} \frac{4 N_{b}}{\xi_{b}^{4}} \int_{0}^{r} d y \int_{y}^{r} d z z y \\
& {\left[K_{0}(y) K_{0}(z) \frac{y^{2}}{2}\left(I_{0}(y)^{2}-I_{1}(y)^{2}\right)\right.} \\
& +\frac{I_{0}(y) I_{0}(z)}{2}\left(\xi_{b}^{2} K_{0}\left(\xi_{b}\right)^{2}-\xi_{b}^{2} K_{1}\left(\xi_{b}\right)^{2}-z^{2} K_{0}(z)^{2}+z^{2} K_{1}(z)^{2}\right) \\
& \left.+\int_{y}^{z} d x x I_{0}(y) K_{0}(z) I_{0}(x) K_{0}(x)\right] .
\end{aligned}
$$

The derivation can be found in Section A.3.2.

Finally, we compare all of the results in Fig. 2.15. We confirm Venturini's result of a divergence between the 1D and 3D models for $\xi_{b}>1$ when $r=0$. However, if we calculate the average over the entire beam, we find that the results are nearly identical for the $1 \mathrm{D}$ and $3 \mathrm{D}$ cases.

(The 1D and 3D models are not identical, though the difference is small. We note that the exact difference depends on the transverse beam distribution. The results given here assume a disc of uniform expectation value. However, a Gaussian transverse distribution results in a difference of a factor of $4 / 3$, as seen in Eqs. 3.62 and 3.63.)

\section{A.2 Longitudinal Space Charge from Shot Noise}

We begin by calculating the LSC for an electron density of form $\lambda \rho(r, \phi, z)$, where $\lambda=N_{b} / L$ is the average linear density over a bunch of $N_{b}$ particles in length $L$. The longitudinal field is given by

$$
E_{z}(x)=\frac{e \lambda}{4 \pi \epsilon_{0}} \int d \boldsymbol{x}^{\prime} G\left(\boldsymbol{x}, \boldsymbol{x}^{\prime}\right) \rho\left(\boldsymbol{x}^{\prime}\right)
$$

with Green's function

$$
\begin{aligned}
G\left(\boldsymbol{x}, \boldsymbol{x}^{\prime}\right) & =\frac{\left(z-z^{\prime}\right) \gamma}{\left[\left(x-x^{\prime}\right)^{2}+\left(y-y^{\prime}\right)^{2}+\gamma^{2}\left(z-z^{\prime}\right)^{2}\right]^{3 / 2}} \\
& =-\frac{i}{\pi \gamma^{2}} \sum_{m=-\infty}^{\infty} e^{i m\left(\phi-\phi^{\prime}\right)} \int_{-\infty}^{\infty} d k e^{i k\left(z-z^{\prime}\right)} k I_{m}\left(\frac{k r_{<}}{\gamma}\right) K_{m}\left(\frac{k r_{>}}{\gamma}\right)
\end{aligned}
$$


Plugging back in to $E_{z}$ we find

$$
E_{z}(x)=\frac{e \lambda}{4 \pi \epsilon_{0}} \int d V^{\prime} \rho\left(r^{\prime}, \phi^{\prime}, z^{\prime}\right) \frac{-i}{\pi \gamma^{2}} \sum_{m=-\infty}^{\infty} e^{i m\left(\phi-\phi^{\prime}\right)} \int_{-\infty}^{\infty} d k e^{i k\left(z-z^{\prime}\right)} k I_{m}\left(\frac{k r_{<}}{\gamma}\right) K_{m}\left(\frac{k r_{>}}{\gamma}\right) .
$$

Taking the Fourier transform, $\tilde{E}_{z}(k)=(2 \pi)^{-1} \int E_{z}(\boldsymbol{x}) e^{-i k^{\prime} z} d z$, gives

$$
\tilde{E}_{z}\left(k^{\prime}\right)=\frac{-i e}{4 \pi \epsilon_{0}} \frac{\lambda}{\pi \gamma^{2}} \sum_{m=-\infty}^{\infty} e^{i m\left(\phi-\phi^{\prime}\right)} \int d V^{\prime} I_{m}^{<} K_{m}^{>} \frac{1}{2 \pi} \int d z e^{-i k^{\prime} z} \int d k e^{-i k z}\left(\rho\left(r^{\prime}, \phi^{\prime}, z^{\prime}\right) k e^{i k z^{\prime}}\right)
$$

with $I_{m}^{<} \equiv I_{m}\left(\frac{k r^{<}}{\gamma}\right)$ and $K_{m}^{>} \equiv K_{m}\left(\frac{k r^{<}}{\gamma}\right)$, and $r^{<}$and $r^{>}$denote the smaller and larger of $r$ and $r^{\prime}$. The two fourier integrals then become an FT-IFT pair and cancel, so we find

$$
\tilde{E}_{z}(k)=-i \frac{e}{4 \pi \epsilon_{0}} \frac{\lambda}{\pi \gamma^{2}} \sum_{m=-\infty}^{\infty} \times \int d V^{\prime} \rho\left(r^{\prime}, \phi^{\prime}, z^{\prime}\right) k e^{i m\left(\phi-\phi^{\prime}\right)} e^{-i k z^{\prime}} I_{m}^{<} K_{m}^{>}
$$

which matches Eq.2 in Marco's paper, and is the starting point for both our 1D and 3D calculations.

\section{A.2.1 1D LSC Model}

We start with the explicit definition of $\rho(\boldsymbol{x})$

$$
\rho_{1 D}(z)= \begin{cases}\frac{L}{N_{b} \pi r_{b}^{2}} \sum_{j}^{N_{b}} \delta\left(z-z_{j}\right) & \text { if } r \leq r_{b} \\ 0 & \text { if } r>r_{b}\end{cases}
$$

where the normalization factor is chosen so that $\int_{-L / 2}^{L / 2} \lambda \rho(z) d V=N_{b}$. Using Eq. A.11, we can write down an expression for the correlation, $\tilde{E}_{k} \tilde{E}_{k}^{*}$. Plugging in for $\rho_{1 D}=\frac{L}{N_{b} \pi r_{b}^{2}} \sum_{j}^{N_{b}} \delta\left(z-z_{j}\right)$ from above we get

$$
\begin{aligned}
& \left\langle\tilde{E}_{z}\left(k_{1}\right) \tilde{E}_{z}^{*}\left(k_{2}\right)\right\rangle=\left(\frac{e}{4 \pi \epsilon_{0}} \frac{\lambda}{\pi \gamma^{2}}\right)^{2} \sum_{m^{\prime}=-\infty}^{\infty} \sum_{m^{\prime \prime}=-\infty}^{\infty} \iint r^{\prime} r^{\prime \prime} d r^{\prime} d \phi^{\prime} d z^{\prime} d r^{\prime \prime} d \phi^{\prime \prime} d z^{\prime \prime}\left(\frac{L}{N_{b} \pi r_{b}^{2}}\right)^{2} \\
& e^{i m^{\prime}\left(\phi_{1}-\phi^{\prime}\right)} e^{-i m^{\prime \prime}\left(\phi_{2}-\phi^{\prime \prime}\right)}\left\langle\sum_{j}^{N_{b}} \sum_{l}^{N_{b}} \delta\left(z^{\prime}-z_{j}\right) \delta\left(z^{\prime \prime}-z_{l}\right) k_{1} k_{2} e^{-i k_{1} z^{\prime}} e^{i k_{2} z^{\prime \prime}}\right\rangle I_{m^{\prime} 1}^{<_{1}^{\prime}} K_{m^{\prime} 1}^{>^{\prime}} I_{m^{\prime \prime} 2}^{<^{\prime \prime}} K_{m^{\prime \prime} 2}^{>^{\prime \prime}}
\end{aligned}
$$

where the Bessel function sub- and super-scripts show, for example, that $I_{m^{\prime} 1}^{<^{\prime}}$ is the $m^{\text {th }}$ Bessel function, and depends on the lesser of $k r_{1} / \gamma$ and $k r^{\prime} / \gamma$. The only dependence on $\phi$ comes from $e^{i m \phi}$, so carrying out the $\phi$ integrals, we are left only with a factor of $2 \pi$ for each of $m^{\prime}, m^{\prime \prime}=0$. Finally, dividing the remaining sums over $j$ and $l$ into coherent and incoherent portions, $\sum_{j}^{N_{b}} \sum_{l}^{N_{b}}=\sum_{j=l}^{N_{b}}+\sum_{j \neq l}^{N_{b}}$, gives

$$
\begin{aligned}
\left\langle\tilde{E}_{z}\left(k_{1}\right) \tilde{E}_{z}^{*}\left(k_{2}\right)\right\rangle= & \left(\frac{e}{4 \pi \epsilon_{0}} \frac{2 \lambda}{\gamma^{2}}\right)^{2}\left(\frac{L}{N_{b} \pi r_{b}^{2}}\right)^{2} \iint r^{\prime} r^{\prime \prime} d r^{\prime} d z^{\prime} d r^{\prime \prime} d z^{\prime \prime} k_{1} k_{2} e^{-i k_{1} z^{\prime}} e^{i k_{2} z^{\prime \prime}} I_{01}^{<^{\prime}} K_{01}^{>^{\prime}} I_{02}^{<^{\prime \prime}} K_{02}^{>^{\prime \prime}} \\
& \times\left\langle\sum_{j=l}^{N_{b}} \delta\left(z^{\prime}-z_{j}\right) \delta\left(z^{\prime \prime}-z_{j}\right)+\sum_{j \neq l}^{N_{b}} \delta\left(z^{\prime}-z_{j}\right) \delta\left(z^{\prime \prime}-z_{l}\right)\right\rangle
\end{aligned}
$$


Integrating over $d z^{\prime}$ and $d z^{\prime \prime}$ takes care of the delta functions to give

$$
\begin{aligned}
\left\langle\tilde{E}_{z}\left(k_{1}\right) \tilde{E}_{z}^{*}\left(k_{2}\right)\right\rangle= & \left(\frac{e}{4 \pi \epsilon_{0}} \frac{2 \lambda}{\gamma^{2}}\right)^{2}\left(\frac{L}{N_{b} \pi r_{b}^{2}}\right)^{2} \iint r^{\prime} r^{\prime \prime} d r^{\prime} d r^{\prime \prime} k_{1} k_{2} I_{01}^{<^{\prime}} K_{01}^{>^{\prime}} I_{02}^{<^{\prime \prime}} K_{02}^{>^{\prime \prime}} \\
& \times\left\langle\sum_{j=l}^{N_{b}} e^{-i z_{j}\left(k_{1}-k_{2}\right)}+\sum_{j \neq l}^{N_{b}} e^{-i\left(k_{1} z_{j}-k_{2} z_{l}\right)}\right\rangle .
\end{aligned}
$$

If we take $k_{1}=k_{2} \neq 0$, we can drop the second sum to find

$$
\left\langle\tilde{E}_{z}\left(k_{1}\right) \tilde{E}_{z}^{*}\left(k_{2}\right)\right\rangle=\left(\frac{e}{2 \pi \epsilon_{0} \gamma^{2} \pi r_{b}^{2}}\right)^{2} N_{b} k_{1}^{2}\left(\int r^{\prime} d r^{\prime} I_{01}^{<} K_{01}^{>}\right)\left(\int r^{\prime \prime} d r^{\prime \prime} I_{02}^{<} K_{02}^{>}\right) .
$$

We can solve the radial integrals by breaking them into 2 pieces, with, for instance, $r_{1}>r^{\prime}$ and $r_{1}<r^{\prime}$. Defining $x \equiv k r^{\prime} / \gamma, \xi_{1}=k r_{1} / \gamma$ we can rewrite each integral as

$$
\int r^{\prime} d r^{\prime} I_{01}^{<^{\prime}} K_{01}^{>^{\prime}}=\frac{\gamma^{2}}{k_{1}^{2}}\left[K_{01}\left(\xi_{1}\right) \int_{0}^{\xi_{1}} d x x I_{0}(x)+I_{01}\left(\xi_{1}\right) \int_{\xi_{1}}^{\xi_{b}} d x x K_{0}(x)\right]
$$

and using $\int x^{\prime} I_{0}\left(x^{\prime}\right) d x^{\prime}=x I_{1}(x), \int x^{\prime} K_{0}\left(x^{\prime}\right) d x^{\prime}=-x K_{1}(x), I_{1}(0)=0$, we have

$$
\int r^{\prime} d r^{\prime} I_{01}^{<^{\prime}} K_{01}^{>^{\prime}}=\frac{\gamma^{2}}{k_{1}^{2}}\left[K_{0}\left(\xi_{1}\right) \xi_{1} I_{1}\left(\xi_{1}\right)+I_{0}\left(\xi_{1}\right)\left[\xi_{1} K_{1}\left(\xi_{1}\right)-\xi_{b} K_{1}\left(\xi_{b}\right)\right]\right] .
$$

Next, we can use the identity $K_{0} I_{1}+I_{0} K_{1}=1 / x$ to find

$$
\int r^{\prime} d r^{\prime} I_{01}^{<^{\prime}} K_{01}^{>^{\prime}}=\frac{\gamma^{2}}{k_{1}^{2}}\left[1-\xi_{b} I_{0}\left(\xi_{1}\right) K_{1}\left(\xi_{b}\right)\right] .
$$

Plugging back in to Eq.A.16 then gives a final result

$$
\left\langle\tilde{E}_{z}\left(k_{1}\right) \tilde{E}_{z}^{*}\left(k_{2}\right)\right\rangle=\left(\frac{e}{2 \pi \epsilon_{0} \gamma \pi r_{b}}\right)^{2} \frac{N_{b}}{\xi_{b}^{2}}\left[1-\xi_{b} I_{0}\left(\xi_{1}\right) K_{1}\left(\xi_{b}\right)\right]\left[1-\xi_{b} I_{0}\left(\xi_{2}\right) K_{1}\left(\xi_{b}\right)\right] .
$$

Taking the special case, $r_{1}, r_{2}=0 \rightarrow \xi_{1}, \xi_{2}=0$,

$$
\left\langle\tilde{E}_{z}\left(k_{1}\right) \tilde{E}_{z}^{*}\left(k_{2}\right)\right\rangle_{r_{1}, r_{2}=0}=\left(\frac{e}{2 \pi^{2} \epsilon_{0} \gamma r_{b}}\right)^{2} N_{b}\left(\frac{1-\xi_{b} K_{1}\left(\xi_{b}\right)}{\xi_{b}}\right)^{2}
$$

recovering the same result as in [25].

\section{A.2.2 3D LSC Model}

We now repeat the method from section A.2.1, this time with a 3D model for $\rho(\boldsymbol{x})$.

$$
\rho_{3 D}(z)= \begin{cases}\frac{L}{N_{b}} \sum_{j}^{N_{b}} \frac{1}{r} \delta\left(r-r_{j}\right) \delta\left(\phi-\phi_{j}\right) \delta\left(z-z_{j}\right) & \text { if } r \leq r_{b}, \\ 0 & \text { if } r>r_{b}\end{cases}
$$

where again the normalization factor is chosen so that $\int \lambda \rho(z) d V=N_{b}$

$$
\Rightarrow \lambda \frac{L}{N_{b}} \sum_{j=1}^{N_{b}} \int \frac{r d r d \phi d z}{r} \delta\left(r-r_{j}\right) \delta\left(\phi-\phi_{j}\right) \delta\left(z-z_{j}\right)=\frac{N_{b} \lambda L}{N_{b}}=N_{b} .
$$


Plugging in to Eq.A.11 with $\rho_{3 D}(z)$ we get

$$
\begin{aligned}
\left\langle\tilde{E}_{z}\left(k_{1}\right)\right. & \left.\tilde{E}_{z}^{*}\left(k_{2}\right)\right\rangle=i i^{*}\left(\frac{-e}{4 \pi \epsilon_{0}} \frac{\lambda}{\pi \gamma^{2}}\right)^{2} \sum_{m^{\prime}} \sum_{m^{\prime \prime}} \iint r^{\prime} r^{\prime \prime} d r^{\prime} d \phi^{\prime} d z^{\prime} d r^{\prime \prime} d \phi^{\prime \prime} d z^{\prime \prime} \\
& \left(\frac{L}{N_{b}}\right)^{2} e^{i m^{\prime}\left(\phi_{1}-\phi^{\prime}\right)} e^{-i m^{\prime \prime}\left(\phi_{2}-\phi^{\prime \prime}\right)} I_{m^{\prime} 1}^{<_{1}^{\prime}} K_{m^{\prime} 1}^{>_{1}^{\prime}} I_{m^{\prime \prime} 2}^{<^{\prime \prime}} K_{m^{\prime \prime} 2}^{>^{\prime \prime}} k_{1} k_{2} e^{-i k_{1} z^{\prime}} e^{i k_{2} z^{\prime \prime}} \\
& \left\langle\sum_{j}^{N_{b}} \sum_{l}^{N_{b}} \frac{1}{r^{\prime} r^{\prime \prime}} \delta\left(r^{\prime}-r_{j}\right) \delta\left(\phi^{\prime}-\phi_{j}\right) \delta\left(z^{\prime}-z_{j}\right) \delta\left(r^{\prime \prime}-r_{l}\right) \delta\left(\phi^{\prime \prime}-\phi_{l}\right) \delta\left(z^{\prime \prime}-z_{l}\right)\right\rangle .
\end{aligned}
$$

Again, dividing the sum into coherent and incoherent portions

$$
\sum_{j}^{N_{b}} \sum_{l}^{N_{b}}=\sum_{j=l}^{N_{b}}+\sum_{j \neq l}^{N_{b}}
$$

we find

$$
\begin{aligned}
\left\langle\tilde{E}_{z}\left(k_{1}\right)\right. & \left.\tilde{E}_{z}^{*}\left(k_{2}\right)\right\rangle=\left(\frac{e}{4 \pi \epsilon_{0}} \frac{\lambda}{\pi \gamma^{2}}\right)^{2}\left(\frac{L}{N_{b}}\right)^{2} \sum_{m^{\prime}} \sum_{m^{\prime \prime}} \iint r^{\prime} r^{\prime \prime} d r^{\prime} d \phi^{\prime} d z^{\prime} d r^{\prime \prime} d \phi^{\prime \prime} d z^{\prime \prime} \\
& \times k_{1} k_{2} e^{-i k_{1} z^{\prime}} e^{i k_{2} z^{\prime \prime}} I_{m^{\prime} 1}^{<^{\prime}} K_{m^{\prime} 1}^{>^{\prime}} I_{m^{\prime \prime} 2}^{<\prime} K_{m^{\prime \prime} 2}^{>^{\prime \prime}} e^{i m^{\prime}\left(\phi_{1}-\phi^{\prime}\right)} e^{-i m^{\prime \prime}\left(\phi_{2}-\phi^{\prime \prime}\right)} \\
& \times\left\langle\sum_{j=l}^{N_{b}} \frac{1}{r^{\prime} r^{\prime \prime}} \delta\left(r^{\prime}-r_{j}\right) \delta\left(r^{\prime \prime}-r_{l}\right) \delta\left(\phi^{\prime}-\phi_{j}\right) \delta\left(\phi^{\prime \prime}-\phi_{l}\right) \delta\left(z^{\prime}-z_{j}\right) \delta\left(z^{\prime \prime}-z_{l}\right)\right\rangle \\
& +\left\langle\sum_{j \neq l}^{N_{b}} \frac{1}{r^{\prime} r^{\prime \prime}} \delta\left(r^{\prime}-r_{j}\right) \delta\left(r^{\prime \prime}-r_{l}\right) \delta\left(\phi^{\prime}-\phi_{j}\right) \delta\left(\phi^{\prime \prime}-\phi_{l}\right) \delta\left(z^{\prime}-z_{j}\right) \delta\left(z^{\prime \prime}-z_{l}\right)\right\rangle .
\end{aligned}
$$

To treat the delta functions in the first term, we use the relation

$$
\left\langle\sum_{i=j}^{N} \delta\left(r-r_{i}\right) \delta\left(r^{\prime}-r_{i}\right)\right\rangle=\left\langle\sum_{i=j}^{N} \delta\left(r-r_{i}\right)\right\rangle \delta\left(r-r^{\prime}\right)=\delta\left(r-r^{\prime}\right)\langle N(r)\rangle
$$

In the case of the 3D distribution, we can use this to rewrite the first term

$$
\begin{aligned}
& \left\langle\sum_{j=l}^{N_{b}} \frac{1}{r^{\prime} r^{\prime \prime}} \delta\left(r^{\prime}-r_{j}\right) \delta\left(r^{\prime \prime}-r_{l}\right) \delta\left(\phi^{\prime}-\phi_{j}\right) \delta\left(\phi^{\prime \prime}-\phi_{l}\right) \delta\left(z^{\prime}-z_{j}\right) \delta\left(z^{\prime \prime}-z_{l}\right)\right\rangle \\
& =\left\langle\frac{\delta\left(r^{\prime}-r^{\prime \prime}\right) \delta\left(\phi^{\prime}-\phi^{\prime \prime}\right) \delta\left(z^{\prime}-z^{\prime \prime}\right)}{r^{\prime \prime}} \sum_{j}^{N_{b}} \frac{\delta\left(r^{\prime}-r_{j}\right) \delta\left(\phi^{\prime}-\phi_{j}\right) \delta\left(z^{\prime}-z_{j}\right)}{r^{\prime}}\right\rangle \\
& =\frac{\delta\left(r^{\prime}-r^{\prime \prime}\right) \delta\left(\phi^{\prime}-\phi^{\prime \prime}\right) \delta\left(z^{\prime}-z^{\prime \prime}\right)}{r^{\prime \prime}}\left\langle\rho\left(r^{\prime}, \phi^{\prime}, z^{\prime}\right)\right\rangle .
\end{aligned}
$$

Returning to Eq.A.26, and using Eq.A.28 to replace one set of delta functions in the first sum, 


$$
\begin{aligned}
\left\langle\tilde{E}_{z}\left(k_{1}\right)\right. & \left.\tilde{E}_{z}^{*}\left(k_{2}\right)\right\rangle=\left(\frac{e}{4 \pi \epsilon_{0}} \frac{\lambda}{\pi \gamma^{2}}\right)^{2}\left(\frac{L}{N_{b}}\right)^{2} \sum_{m^{\prime}} \sum_{m^{\prime \prime}} \iint r^{\prime} r^{\prime \prime} d r^{\prime} d \phi^{\prime} d z^{\prime} d r^{\prime \prime} d \phi^{\prime \prime} d z^{\prime \prime} \\
& \times k_{1} k_{2} e^{-i k_{1} z^{\prime}} e^{i k_{2} z^{\prime \prime}} I_{m^{\prime} 1}^{<^{\prime}} K_{m^{\prime} 1}^{>_{1}^{\prime}} I_{m^{\prime \prime} 2}^{<_{2}} K_{m^{\prime \prime} 2}^{>} e^{i m^{\prime}\left(\phi_{1}-\phi^{\prime}\right)} e^{-i m^{\prime \prime}\left(\phi_{2}-\phi^{\prime \prime}\right)} \\
& \times \frac{\delta\left(r^{\prime}-r^{\prime \prime}\right) \delta\left(\phi^{\prime}-\phi^{\prime \prime}\right) \delta\left(z^{\prime}-z^{\prime \prime}\right)}{r^{\prime \prime}}\left\langle\rho\left(r^{\prime}, \phi^{\prime}, z^{\prime}\right)\right\rangle \\
& +\left\langle\sum_{j \neq l}^{N_{b}} \frac{1}{r^{\prime} r^{\prime \prime}} \delta\left(r^{\prime}-r_{j}\right) \delta\left(r^{\prime \prime}-r_{l}\right) \delta\left(\phi^{\prime}-\phi_{j}\right) \delta\left(\phi^{\prime \prime}-\phi_{l}\right) \delta\left(z^{\prime}-z_{j}\right) \delta\left(z^{\prime \prime}-z_{l}\right)\right\rangle
\end{aligned}
$$

plugging in for $\langle\rho\rangle=N_{b} / \pi r_{b}^{2} L$, canceling factors of $r^{\prime \prime}$ and integrating over the delta functions, we find

$$
\begin{aligned}
\left\langle\tilde{E}_{z}\left(k_{1}\right) \tilde{E}_{z}^{*}\left(k_{2}\right)\right\rangle= & \left(\frac{e}{4 \pi \epsilon_{0}} \frac{\lambda}{\pi \gamma^{2}}\right)^{2}\left(\frac{L}{N_{b}}\right)^{2} \sum_{m^{\prime}} \sum_{m^{\prime \prime}} \int r^{\prime} d r^{\prime} d \phi^{\prime} d z^{\prime} k_{1} k_{2} I_{m^{\prime} 1}^{<} K_{m^{\prime} 1}^{>_{1}^{\prime}} I_{m^{\prime \prime} 2}^{<\prime \prime} K_{m^{\prime \prime} 2}^{>^{\prime \prime}} e^{i m^{\prime}\left(\phi_{1}-\phi^{\prime}\right)} \\
& \times \frac{N_{b}}{\pi r_{b}^{2} L} e^{-i m^{\prime \prime}\left(\phi_{2}-\phi^{\prime}\right)} e^{-i z^{\prime}\left(k_{1}-k_{2}\right)} \delta_{r^{\prime}, r^{\prime \prime}} \delta_{\phi^{\prime}, \phi^{\prime \prime}} \delta_{z^{\prime}, z^{\prime \prime}} \\
& +\left\langle\sum_{j \neq l}^{N_{b}} \frac{1}{r^{\prime}} \delta\left(r^{\prime}-r_{j}\right) \delta\left(\phi^{\prime}-\phi_{j}\right) \delta\left(z^{\prime}-z_{j}\right) e^{-i m^{\prime \prime}\left(\phi_{2}-\phi_{l}\right)} e^{-i k_{1} z^{\prime}+i k_{2} z_{l}}\right\rangle
\end{aligned}
$$

where the $\delta_{r^{\prime}, r^{\prime \prime}}$ term indicates that all 4 Bessel functions for the first term have arguments that depend only on $r^{\prime}$. Integrating over $z^{\prime}$, we find that the first term only contributes if $k_{1}=k_{2}$, and the second term only contributes if $k_{1}=0$. With the assumption $k_{1}, k_{2} \neq 0$, we drop the second term and pick up a factor of $L$ from the first term.

$$
\begin{aligned}
\left\langle\tilde{E}_{z}\left(k_{1}\right) \tilde{E}_{z}^{*}\left(k_{2}\right)\right\rangle= & \left(\frac{e}{4 \pi \epsilon_{0}} \frac{\lambda k}{\pi \gamma^{2}}\right)^{2}\left(\frac{L}{N_{b}}\right)^{2} \frac{N_{b}}{\pi r_{b}^{2}} \sum_{m^{\prime}} \sum_{m^{\prime \prime}} \int r^{\prime} d r^{\prime} d \phi^{\prime} d z^{\prime} \\
& \times I_{m^{\prime} 1}^{<_{1}^{\prime}} K_{m^{\prime} 1}^{>^{\prime}} I_{m^{\prime \prime} 2}^{<^{\prime}} K_{m^{\prime \prime} 2}^{>^{\prime}} e^{i\left(m^{\prime} \phi_{1}-m^{\prime \prime} \phi_{2}\right)} e^{-i \phi^{\prime}\left(m^{\prime}-m^{\prime \prime}\right)} \delta_{k_{1}, k_{2}} .
\end{aligned}
$$

Finally, integrating over $\phi^{\prime}$, we get a factor of $2 \pi$ for terms with $m^{\prime}=m^{\prime \prime}$, and all other terms vanish.

$$
\left\langle\tilde{E}_{z}\left(k_{1}\right) \tilde{E}_{z}^{*}\left(k_{2}\right)\right\rangle=\left(\frac{e}{4 \pi \epsilon_{0}} \frac{\lambda k}{\pi \gamma^{2}}\right)^{2}\left(\frac{L}{N_{b}}\right)^{2} \frac{2 \pi L N_{b}}{\pi r_{b}^{2} L} \delta_{k_{1}, k_{2}} \sum_{m^{\prime}} e^{i m^{\prime}\left(\phi_{1}-\phi_{2}\right)} \int r^{\prime} d r^{\prime} I_{m^{\prime} 1}^{<\prime} K_{m^{\prime} 1}^{>^{\prime}} I_{m^{\prime} 2}^{<^{\prime}} K_{m^{\prime} 2}^{>^{\prime}} .
$$

Combining factors and rewriting the arguments of the Bessel functions, we're left with only the $r^{\prime}$ integral

$$
\begin{aligned}
\left\langle\tilde{E}_{z}\left(k_{1}\right) \tilde{E}_{z}^{*}\left(k_{2}\right)\right\rangle= & \left(\frac{e}{4 \pi^{2} \epsilon_{0} \gamma^{2}}\right)^{2} \frac{2 k_{1}^{2} N_{b}}{r_{b}^{2}} \delta_{k_{1}, k_{2}} \sum_{m^{\prime}} e^{i m^{\prime}\left(\phi_{1}-\phi_{2}\right)} \\
& \int r^{\prime} d r^{\prime} I_{m^{\prime}}\left(\frac{k r_{1}^{<}}{\gamma}\right) K_{m^{\prime}}\left(\frac{k r_{1}^{>}}{\gamma}\right) I_{m^{\prime}}\left(\frac{k r_{2}^{<}}{\gamma}\right) K_{m^{\prime}}\left(\frac{k r_{2}^{>}}{\gamma}\right) .
\end{aligned}
$$

As before, we solve the $r^{\prime}$ integral by breaking it into parts. This time we need 3 sections: $r^{\prime}<r 1, r 2$; $r 1<r^{\prime}<r 2$; and $r 1, r 2<r^{\prime}$. (In the 3rd case, it is also implied that $r^{\prime}<r_{b}$, outside of which the beam does not exist.)

$$
\begin{aligned}
\int_{0}^{r_{b}} r^{\prime} d r^{\prime} I_{m 1} K_{m 1} I_{m 2} K_{m 2} & =K_{m}\left(\frac{k r_{1}}{\gamma}\right) K_{m}\left(\frac{k r_{2}}{\gamma}\right) \int_{0}^{r_{1}} r^{\prime} d r^{\prime} I_{m}\left(\frac{k r^{\prime}}{\gamma}\right)^{2} \\
& +I_{m}\left(\frac{k r_{1}}{\gamma}\right) K_{m}\left(\frac{k r_{2}}{\gamma}\right) \int_{r_{1}}^{r_{2}} r^{\prime} d r^{\prime} I_{m}\left(\frac{k r^{\prime}}{\gamma}\right) K_{m}\left(\frac{k r^{\prime}}{\gamma}\right) \\
& +I_{m}\left(\frac{k r_{1}}{\gamma}\right) I_{m}\left(\frac{k r_{2}}{\gamma}\right) \int_{r_{2}}^{r_{b}} r^{\prime} d r^{\prime} K_{m}\left(\frac{k r^{\prime}}{\gamma}\right)^{2}
\end{aligned}
$$


Changing variables to $x \equiv k r^{\prime} / \gamma$, and defining $\xi=k r / \gamma, \xi_{1}=k r_{1} / \gamma$, etc.

$$
\begin{aligned}
\int_{0}^{r_{b}} r^{\prime} d r^{\prime} I_{m 1} K_{m 1} I_{m 2} K_{m 2} & =K_{m}\left(\xi_{1}\right) K_{m}\left(\xi_{2}\right) \int_{0}^{\xi_{1}} x d x \frac{\gamma^{2}}{k^{2}} I_{m}(x)^{2} \\
& +I_{m}\left(\xi_{1}\right) K_{m}\left(\xi_{2}\right) \int_{\xi_{1}}^{\xi_{2}} x d x \frac{\gamma^{2}}{k^{2}} I_{m}(x) K_{m}(x) \\
& +I_{m}\left(\xi_{1}\right) I_{m}\left(\xi_{2}\right) \int_{\xi_{2}}^{\xi_{b}} x d x \frac{\gamma^{2}}{k^{2}} K_{m}(x)^{2} .
\end{aligned}
$$

Finally, taking the simpler case of $r_{1}, r_{2}=0$, the first two integrals vanish, $I_{0}(0) \rightarrow 1$, and $m=0$ giving

$$
\int_{0}^{r_{b}} r^{\prime} d r^{\prime} I_{01} K_{01} I_{02} K_{02}=\frac{\gamma^{2}}{k^{2}} \int_{0}^{\xi_{b}} d x x K_{0}(x)^{2}
$$

which can be solved using

$$
\int^{\xi_{b}} d x x K_{0}(x)^{2}=\frac{\xi_{b}^{2}\left[K_{0}^{2}\left(\xi_{b}\right)-K_{1}^{2}\left(\xi_{b}\right)\right]}{2}
$$

Evaluating at $\xi_{b}=0$, the first term is 0 (since $K_{0}(\xi) \propto \ln (\xi)$ for small $\xi$ ), and the second term is just -1 (since $K_{1}(\xi) \propto 1 / \xi$ for small $\xi$ ). So we have

$$
\int_{0}^{r_{b}} r^{\prime} d r^{\prime} I_{01} K_{01} I_{02} K_{02}=\frac{\gamma^{2}}{k^{2}} \frac{\xi_{b}^{2}\left[1+K_{0}^{2}\left(\xi_{b}\right)-K_{1}^{2}\left(\xi_{b}\right)\right]}{2} .
$$

Plugging all this back in to Eq.A.33 gives

$$
\left\langle\tilde{E}_{z}\left(k_{1}\right) \tilde{E}_{z}^{*}\left(k_{2}\right)\right\rangle_{r_{1}, r_{2}=0}=\left(\frac{e}{2 \pi^{2} \epsilon_{0} \gamma r_{b}}\right)^{2} N_{b} \frac{1+\xi_{b}^{2}\left[K_{0}^{2}\left(\xi_{b}\right)-K_{1}^{2}\left(\xi_{b}\right)\right]}{4} \delta_{k_{1}, k_{2}}
$$

as in Eq. (11) of [25].

Finally, we note that this result can be generalized for arbitrary $r, r^{\prime}$, by substituting Eq.A.35 for the penultimate factor in Eq.A.39. For example, taking the case $r_{1}=0, r_{2} \neq 0$, gives

$$
\begin{aligned}
\int_{0}^{r_{b}} r^{\prime} d r^{\prime} I_{01} K_{01} I_{02} K_{02}= & K_{0}\left(\xi_{1}\right) K_{0}\left(\xi_{2}\right) \int_{0}^{\xi_{1}} x d x \frac{\gamma^{2}}{k^{2}} I_{0}(x)^{2} \\
& +I_{0}\left(\xi_{1}\right) K_{0}\left(\xi_{2}\right) \int_{\xi_{1}}^{\xi_{2}} x d x \frac{\gamma^{2}}{k^{2}} I_{0}(x) K_{0}(x) \\
& +I_{0}\left(\xi_{1}\right) I_{0}\left(\xi_{2}\right) \int_{\xi_{2}}^{\xi_{b}} x d x \frac{\gamma^{2}}{k^{2}} K_{0}(x)^{2} \\
= & \frac{\gamma^{2}}{k^{2}}\left(K_{0}\left(\xi_{2}\right) \int_{0}^{\xi_{2}} x I_{0}(x) K_{0}(x) d x+I_{0}\left(\xi_{2}\right) \int_{\xi_{2}}^{\xi_{b}} x K_{0}(x)^{2} d x\right)
\end{aligned}
$$

Replacing the final factor in Eq.A.39 with this result and using Venturini's shorthand notation $\tilde{E}_{z}\left(k_{1}, r_{1}\right)=$ $\tilde{E}_{k}\left(r_{1}\right)$ we have

$$
\left\langle\tilde{E}_{k}(0) \tilde{E}_{k}^{*}(r)\right\rangle=\left(\frac{e}{2 \pi^{2} \epsilon_{0} \gamma r_{b}}\right)^{2} \frac{N_{b}}{2}\left[K_{0}(\xi) \int_{0}^{\xi} x I_{0}(x) K_{0}(x) d x+I_{0}(\xi) \int_{\xi}^{\xi_{b}} x K_{0}(x)^{2} d x\right]
$$

which matches Eq.(12) of [25]. 


\section{A.3 Transverse Average}

Venturini's paper gives the expected energy modulation experienced at the center of the bunch $(r=0)$. However, for our bunching calculations, we are interested in finding the transverse average, integrating $\left\langle\tilde{E}\left(r_{1}\right) \tilde{E}\left(r_{2}\right)^{*}\right\rangle$ over $r_{1}, r_{2}, \phi_{1}, \phi_{2}$.

\section{A.3.1 Transverse Average: 1D Case}

First, in the 1D case, we start from Eq.A.20,

$$
\begin{aligned}
\left\langle\tilde{E}_{k_{1}}\left(r_{1}\right) \tilde{E}_{k_{2}}^{*}\left(r_{2}\right)\right\rangle & =\left(\frac{e}{2 \pi \epsilon_{0} \gamma^{2} \pi r_{b}^{2}}\right)^{2} N_{b} k_{1}^{2} \int r^{\prime} d r^{\prime} I_{01}^{<^{\prime}} K_{01}^{>^{\prime}} \int r^{\prime \prime} d r^{\prime \prime} I_{02}^{<^{\prime \prime}} K_{02}^{>^{\prime \prime}} \delta_{k_{1}, k_{2}} \\
& =\left(\frac{e}{2 \pi \epsilon_{0} \gamma \pi r_{b}}\right)^{2} \frac{N_{b}}{\xi_{b}^{2}}\left[1-\xi_{b} I_{0}\left(\xi_{1}\right) K_{1}\left(\xi_{b}\right)\right]\left[1-\xi_{b} I_{0}\left(\xi_{2}\right) K_{1}\left(\xi_{b}\right)\right]
\end{aligned}
$$

where we've again made the substitution $\xi_{1} \equiv k r_{1} / \gamma$. Rather than looking just at the beam center, we want to find the average value over all $r_{1}, r_{2},\left(\pi r_{b}^{2}\right)^{-2} \iint r_{1} r_{2} d r_{1} d r_{2} d \phi_{1} d \phi_{2}\left\langle\tilde{E} \tilde{E}^{*}\right\rangle$. The $\phi$ integrals just give a factor of $4 \pi^{2}$. The $r_{1}$ and $r_{2}$ integrals can be separated, so we evaluate them independently

$$
\int_{0}^{r_{b}} r_{1} d r_{1} \int r^{\prime} d r^{\prime} I_{01}^{<^{\prime}} K_{01}^{>^{\prime}}=\frac{\gamma^{4}}{k^{4}} \int_{0}^{\xi_{b}} x d x\left[1-\xi_{b} I_{0}(x) K_{1}\left(\xi_{b}\right)\right] .
$$

Integrating over $\phi$ and plugging in the result from Eq.A.43 gives

$$
\begin{aligned}
\left(\frac{1}{\pi r_{b}^{2}}\right)^{2} & \int r_{1} d r_{1} d \phi_{1} \int r_{2} d r_{2} d \phi_{2}\left\langle\tilde{E}_{k_{1}}\left(r_{1}\right) \tilde{E}_{k_{2}}^{*}\left(r_{2}\right)\right\rangle \\
& =\left(\frac{2 \pi}{\pi r_{b}^{2}}\right)^{2}\left(\frac{e}{2 \pi \epsilon_{0} \gamma^{2} \pi r_{b}^{2}}\right)^{2} N_{b} k^{2}\left(\frac{\gamma^{4}}{k^{4}} \int_{0}^{\xi_{b}} x d x\left[1-\xi_{b} I_{0}(x) K_{1}\left(\xi_{b}\right)\right]\right)^{2} \\
& =\left(\frac{e}{2 \pi \epsilon_{0} \gamma \pi r_{b}}\right)^{2} 4 N_{b}\left(\frac{\gamma}{k r_{b}}\right)^{6}\left(\frac{\xi_{b}^{2}}{2}-\xi_{b}^{2} K_{1}\left(\xi_{b}\right) I_{1}\left(\xi_{b}\right)\right)^{2}
\end{aligned}
$$

and we find

$$
\left\langle\tilde{E}_{k} \tilde{E}_{k}^{*}\right\rangle_{1 D \perp}=\left(\frac{e}{2 \pi^{2} \epsilon_{0} \gamma r_{b}}\right)^{2} \frac{4 N_{b}}{\xi_{b}^{2}}\left(\frac{1}{2}-K_{1}\left(\xi_{b}\right) I_{1}\left(\xi_{b}\right)\right)^{2}
$$

\section{A.3.2 Transverse Average: 3D Case, Numerical Approach}

We can follow the same method for the 3D case, though the answer will be messier due to the inseparability of the $r_{1}$ and $r_{2}$ integrals. Starting from Eq.A.33

$$
\begin{aligned}
\left\langle\tilde{E}_{k_{1}}\left(r_{1}\right) \tilde{E}_{k_{2}}^{*}\left(r_{2}\right)\right\rangle= & \left(\frac{e}{4 \pi^{2} \epsilon_{0} \gamma^{2}}\right)^{2} \frac{2 k_{1}^{2} N_{b}}{r_{b}^{2}} \delta_{k_{1}, k_{2}} \sum_{m^{\prime}} e^{i m^{\prime}\left(\phi_{1}-\phi_{2}\right)} \\
& \int r^{\prime} d r^{\prime} I_{m^{\prime}}\left(\frac{k r_{1}^{<}}{\gamma}\right) K_{m^{\prime}}\left(\frac{k r_{1}^{>}}{\gamma}\right) I_{m^{\prime}}\left(\frac{k r_{2}^{<}}{\gamma}\right) K_{m^{\prime}}\left(\frac{k r_{2}^{>}}{\gamma}\right)
\end{aligned}
$$


we then average transversely by integrating over $r_{1}, r_{2}, \phi_{1}$, and $\phi_{2}$

$$
\begin{aligned}
\left\langle\left\langle\tilde{E}_{k_{1}} \tilde{E}_{k_{2}}^{*}\right\rangle\right\rangle_{3 D \perp} & =\left(\frac{e}{4 \pi^{2} \epsilon_{0} \gamma^{2}}\right)^{2} \frac{2 k_{1}^{2} N_{b}}{r_{b}^{2}} \delta_{k_{1}, k_{2}}\left(\frac{1}{\pi r_{b}^{2}}\right)^{2} \int_{0}^{2 \pi} \int_{0}^{2 \pi} d \phi_{1} d \phi_{2} \sum_{m^{\prime}} e^{i m^{\prime}\left(\phi_{1}-\phi_{2}\right)} \\
& \int_{0}^{r_{b}} r_{1} d r_{1} \int_{0}^{r_{b}} r_{2} d r_{2} \int r^{\prime} d r^{\prime} I_{m^{\prime}}\left(\frac{k r_{1}^{<}}{\gamma}\right) K_{m^{\prime}}\left(\frac{k r_{1}^{>}}{\gamma}\right) I_{m^{\prime}}\left(\frac{k r_{2}^{<}}{\gamma}\right) K_{m^{\prime}}\left(\frac{k r_{2}^{>}}{\gamma}\right) .
\end{aligned}
$$

The $\phi$ integrals set $m=0$ (all other terms disappear when we do the average) and pick up a factor of $4 \pi^{2}$. The $r_{1}, r_{2}$ integrals are messy, because the limits of the $r^{\prime}$ integral are determined by the values of $r_{1}$ and $r_{2}$. Since $r_{1}$ and $r_{2}$ are interchangeable, we can rewrite the integrals as

$$
\int_{0}^{r_{b}} r_{1} d r_{1} \int_{0}^{r_{b}} r_{2} d r_{2}=2 \int_{0}^{r_{b}} r_{2} d r_{2} \int_{0}^{r_{2}} r_{1} d r_{1}
$$

so that $r_{1}$ is always less than $r_{2}$. Using this result and plugging in Eq.A.34 for the $r^{\prime}$ integral gives

$$
\begin{aligned}
& \left\langle\left\langle\tilde{E}_{k_{1}} \tilde{E}_{k_{2}}^{*}\right\rangle\right\rangle_{3 D \perp}=\left(\frac{2}{r_{b}^{2}}\right)^{2}\left(\frac{e}{4 \pi^{2} \epsilon_{0} \gamma^{2}}\right)^{2} \frac{2 k^{2} N_{b}}{r_{b}^{2}} \delta_{k_{1}, k_{2}} 2 \int_{0}^{r_{b}} r_{2} d r_{2} \int_{0}^{r_{2}} r_{1} d r_{1} \\
& \frac{\gamma^{2}}{k^{2}}\left[K_{0}\left(\xi_{1}\right) K_{0}\left(\xi_{2}\right) \int_{0}^{\xi_{1}} x d x I_{0}(x)^{2}+I_{0}\left(\xi_{1}\right) K_{0}\left(\xi_{2}\right) \int_{\xi_{1}}^{\xi_{2}} x d x I_{0}(x) K_{0}(x)+I_{0}\left(\xi_{1}\right) I_{0}\left(\xi_{2}\right) \int_{\xi_{2}}^{\xi_{b}} x d x K_{0}(x)^{2}\right] .
\end{aligned}
$$

Making the same substitution we used for the $r^{\prime}$ integral, we can define $y \equiv k r_{1} / \gamma=\xi_{1}$, and $z \equiv k r_{2} / \gamma=\xi_{2}$ to find

$$
\begin{aligned}
& \left\langle\left\langle\tilde{E}_{k_{1}} \tilde{E}_{k_{2}}^{*}\right\rangle\right\rangle_{3 D \perp}=\left(\frac{e}{2 \pi^{2} \epsilon_{0} \gamma r_{b}}\right)^{2} N_{b} \frac{4}{\xi_{b}^{4}} \int_{0}^{\xi_{b}} d z \int_{0}^{z} d y \\
& z y\left[K_{0}(y) K_{0}(z) \int_{0}^{y} d x x I_{0}(x)^{2}+I_{0}(y) K_{0}(z) \int_{y}^{z} d x x I_{0}(x) K_{0}(x)+I_{0}(y) I_{0}(z) \int_{z}^{\xi_{b}} d x x K_{0}(x)^{2}\right] \delta_{k_{1}, k_{2}} .
\end{aligned}
$$

To simplify the integration, we can evaluate the first and third $x$ integrals analytically. Using $\int d x x K_{0}(x)^{2}=$ $\frac{x^{2}}{2}\left[K_{0}(x)^{2}-K_{1}(x)^{2}\right]$ and $\int d x x I_{0}(x)^{2}=\frac{x^{2}}{2}\left[I_{0}(x)^{2}-I_{1}(x)^{2}\right]$, and $x I_{0}(0)=I_{1}(0)=0$, we have

$$
\begin{aligned}
& \left\langle\left\langle\tilde{E}_{k_{1}} \tilde{E}_{k_{2}}^{*}\right\rangle\right\rangle_{3 D \perp}=\left(\frac{e}{2 \pi^{2} \epsilon_{0} \gamma r_{b}}\right)^{2} \frac{4 N_{b}}{\xi_{b}^{4}} \delta_{k_{1}, k_{2}} \int_{0}^{\xi_{b}} d z \int_{0}^{z} d y z y \times\left[\int_{y}^{z} d x x I_{0}(y) K_{0}(z) I_{0}(x) K_{0}(x)+\right. \\
& \left.K_{0}(y) K_{0}(z) \frac{y^{2}}{2}\left(I_{0}(y)^{2}-I_{1}(y)^{2}\right)+\frac{I_{0}(y) I_{0}(z)}{2}\left(\xi_{b}^{2} K_{0}\left(\xi_{b}\right)^{2}-\xi_{b}^{2} K_{1}\left(\xi_{b}\right)^{2}-z^{2} K_{0}(z)^{2}+z^{2} K_{1}(z)^{2}\right)\right] .
\end{aligned}
$$

We then can compare our result to that of the 1D case,

$$
\left\langle\left\langle\tilde{E}_{k_{1}} \tilde{E}_{k_{2}}^{*}\right\rangle\right\rangle_{1 D \perp}=\left(\frac{e}{2 \pi^{2} \epsilon_{0} \gamma r_{b}}\right)^{2} \frac{4 N_{b}}{\xi_{b}^{2}}\left(\frac{1}{2}-K_{1}\left(\xi_{b}\right) I_{1}\left(\xi_{b}\right)\right)^{2} \delta_{k_{1}, k_{2}} .
$$

Evaluating both numerically, we see they're nearly identical (Fig. 2.15)! We can also check our math by integrating numerically in the limit $r_{1}, r_{2} \rightarrow 0$, and see that we reproduce our previous result. 


\section{A.3.3 Transverse Average: 3D Case, Analytical Approach}

Having seen numerically that the 1D and 3D models match, we now try to show the same analytically. We repeat this calculation for the 3D model, this time starting from the earlier Eq.A.29 and again integrating over transverse coordinates $\phi_{1}, \phi_{2}, r_{1}, r_{2}$.

$$
\begin{aligned}
\left\langle\left\langle\tilde{E}_{k_{1}} \tilde{E}_{k_{2}}^{*}\right\rangle\right\rangle_{3 D \perp} & =\left(\frac{1}{\pi r_{b}^{2}}\right)^{2}\left(\frac{e}{4 \pi \epsilon_{0}} \frac{\lambda}{\pi \gamma^{2}}\right)^{2}\left(\frac{L}{N_{b}}\right)^{2} \sum_{m^{\prime}} \sum_{m^{\prime \prime}} \\
& \iint r_{1} r_{2} d r_{1} d \phi_{1} d r_{2} d \phi_{2} \iint r^{\prime} r^{\prime \prime} d r^{\prime} d \phi^{\prime} d z^{\prime} d r^{\prime \prime} d \phi^{\prime \prime} d z^{\prime \prime} \\
& \times k_{1} k_{2} e^{-i k_{1} z^{\prime}} e^{i k_{2} z^{\prime \prime}} I_{m^{\prime} 1}^{<_{1}^{\prime}} K_{m^{\prime} 1}^{>_{1}^{\prime}} I_{m^{\prime \prime} 2}^{<\prime} K_{m^{\prime \prime} 2}^{>^{\prime \prime}} e^{i m^{\prime}\left(\phi_{1}-\phi^{\prime}\right)} e^{-i m^{\prime \prime}\left(\phi_{2}-\phi^{\prime \prime}\right)} \\
& \times\left(\frac{\delta\left(r^{\prime}-r^{\prime \prime}\right) \delta\left(\phi^{\prime}-\phi^{\prime \prime}\right) \delta\left(z^{\prime}-z^{\prime \prime}\right)}{r^{\prime \prime}}\left\langle\rho\left(r^{\prime}, \phi^{\prime}, z^{\prime}\right)\right\rangle\right. \\
& \left.+\left\langle\sum_{j \neq l}^{N_{b}} \frac{1}{r^{\prime} r^{\prime \prime}} \delta\left(r^{\prime}-r_{j}\right) \delta\left(r^{\prime \prime}-r_{l}\right) \delta\left(\phi^{\prime}-\phi_{j}\right) \delta\left(\phi^{\prime \prime}-\phi_{l}\right) \delta\left(z^{\prime}-z_{j}\right) \delta\left(z^{\prime \prime}-z_{l}\right)\right\rangle\right) .
\end{aligned}
$$

The 1D and 3D LSC expressions (Eq. A.21, Eq. A.39) are different primarily because of these $\delta$ functions, which in the 3D case combine the transverse dependence of $\tilde{E}$ and $\tilde{E}^{*}$ into one integral. (This is what leads to the coherence length mathematically.) The transverse averaging seems to wash out this coherence. (Instead of looking at two points, and the coherence between them, we look at all transverse points.) Mathematically, we can take advantage of the transverse averaging integrals to split up the $r^{\prime}$ and $r^{\prime \prime}$ integrals by integrating over $\phi_{1}, \phi_{2}, r_{1}, r_{2}$ before evaluating the $\delta$ functions. We start with $\phi_{1}$ and $\phi_{2}$, giving

$$
\begin{aligned}
\left\langle\left\langle\tilde{E}_{k_{1}} \tilde{E}_{k_{2}}^{*}\right\rangle\right\rangle_{3 D \perp} & =\left(\frac{1}{\pi r_{b}^{2}}\right)^{2}\left(\frac{e}{4 \pi \epsilon_{0}} \frac{\lambda}{\pi \gamma^{2}}\right)^{2}\left(\frac{L}{N_{b}}\right)^{2}\left(4 \pi^{2}\right) 2 \pi \iint r^{\prime} d r^{\prime} r^{\prime \prime} d r^{\prime \prime} \\
& \frac{\delta\left(r^{\prime}-r^{\prime \prime}\right)}{r^{\prime \prime}} \delta_{k_{1}, k_{2}} \frac{N_{b}}{\pi r_{b}^{2} L} k_{1} k_{2} \int r_{1} d r_{1} I_{01}^{<^{\prime}} K_{01}^{>^{\prime}} \int r_{2} d r_{2} I_{02}^{<^{\prime \prime}} K_{02}^{>^{\prime \prime}}
\end{aligned}
$$

where we've also dropped the (non-contributing) coherent term and carried out the now trivial $\phi^{\prime}, \phi^{\prime \prime}, z^{\prime}, z^{\prime \prime}$ integrals. Next, we integrate over $r_{1}, r_{2}$. This keeps the $r^{\prime}$ and $r^{\prime \prime}$ integrals separate, as in the 1D case, leaving us integrals (similar to their 1D counterparts) of the form

$$
\begin{aligned}
\int r_{1} d r_{1} I_{01}^{<^{\prime}} K_{01}^{>^{\prime}} & =\frac{\gamma^{2}}{k_{1}^{2}}\left[K_{01}\left(\xi^{\prime}\right) \int_{0}^{\xi^{\prime}} d x x I_{0}(x)+I_{01}\left(\xi^{\prime}\right) \int_{\xi^{\prime}}^{\xi_{b}} d x x K_{0}(x)\right] \\
& =\frac{\gamma^{2}}{k_{1}^{2}}\left[1-\xi_{b} I_{0}\left(\xi^{\prime}\right) K_{1}\left(\xi_{b}\right)\right] .
\end{aligned}
$$


Plugging back in above and finally integrating over $r^{\prime}, r^{\prime \prime}$, we find

$$
\begin{aligned}
\left\langle\tilde{E}_{k} \tilde{E}_{k}^{*}\right\rangle_{3 D \perp} & =\left(\frac{1}{\pi r_{b}^{2}}\right)^{2}\left(\frac{e}{4 \pi \epsilon_{0}} \frac{\lambda}{\pi \gamma^{2}}\right)^{2}\left(\frac{L}{N_{b}}\right)^{2}\left(4 \pi^{2}\right) 2 \pi \iint r^{\prime} d r^{\prime} r^{\prime \prime} d r^{\prime \prime} \\
& \frac{\delta\left(r^{\prime}-r^{\prime \prime}\right)}{r^{\prime \prime}} \frac{N_{b}}{\pi r_{b}^{2} L} k k\left(\frac{\gamma^{2}}{k k}\right)^{2}\left[1-\xi_{b} I_{0}\left(\xi^{\prime}\right) K_{1}\left(\xi_{b}\right)\right]\left[1-\xi_{b} I_{0}\left(\xi^{\prime \prime}\right) K_{1}\left(\xi_{b}\right)\right] \\
& =\left(\frac{1}{\pi r_{b}^{2}}\right)^{2}\left(\frac{e}{2 \epsilon_{0} \pi \gamma^{2}}\right)^{2} 2 \frac{N_{b}}{r_{b}^{2}} k^{2}\left(\frac{\gamma}{k}\right)^{4} \int_{0}^{r_{b}} r^{\prime} d r^{\prime}\left[1-\xi_{b} I_{0}\left(\xi^{\prime}\right) K_{1}\left(\xi_{b}\right)\right]^{2} \\
& =\left(\frac{e}{2 \pi^{2} \epsilon_{0} \gamma r_{b}}\right)^{2} N_{b} \frac{2}{\xi^{4}} \int_{0}^{\xi_{b}} d x\left[x-2 \xi_{b} K_{1}\left(\xi_{b}\right) x I_{0}(x)+\xi_{b}^{2} K_{1}\left(\xi_{b}\right)^{2} x I_{0}(x)^{2}\right] \\
& =\left(\frac{e}{2 \pi^{2} \epsilon_{0} \gamma r_{b}}\right)^{2} N_{b} \frac{4}{\xi^{2}}\left[\frac{1}{4}-K_{1}\left(\xi_{b}\right) I_{1}\left(\xi_{b}\right)+\frac{\xi_{b}^{2}}{4} K_{1}\left(\xi_{b}\right)^{2}\left(I_{0}\left(\xi_{b}\right)^{2}-I_{1}\left(\xi_{b}\right)^{2}\right)\right]
\end{aligned}
$$

Getting close! Now, using the recurrence formula $I_{m}(x)=2(m+1) I_{m+1}(x) / x+I_{m+2}(x)$, we find

$$
x^{2} I_{0}(x)^{2}=4 I_{1}(x)^{2}+\left[4 x I_{1}(x) I_{2}(x)+x^{2} I_{2}(x)^{2}\right]
$$

so our transverse average can be written

$$
\left\langle\tilde{E}_{k} \tilde{E}_{k}^{*}\right\rangle_{3 D \perp}=\left(\frac{e}{2 \pi^{2} \epsilon_{0} \gamma r_{b}}\right)^{2} N_{b} \frac{4}{\xi^{2}}\left[\frac{1}{4}-K_{1}\left(\xi_{b}\right) I_{1}\left(\xi_{b}\right)+K_{1}\left(\xi_{b}\right)^{2} I_{1}\left(\xi_{b}\right)^{2}+\delta\left(\xi_{b}\right)\right]
$$

with

$$
\delta(x) \equiv K_{1}\left(\xi_{b}\right)^{2}\left(\xi_{b} I_{1}\left(\xi_{b}\right) I_{2}\left(\xi_{b}\right)+\frac{\xi_{b}^{2}}{4}\left(I_{2}\left(\xi_{b}\right)^{2}-I_{1}\left(\xi_{b}\right)^{2}\right)\right) .
$$

Rewriting this to match the 1D case:

$$
\left\langle\tilde{E}_{k} \tilde{E}_{k}^{*}\right\rangle_{3 D \perp}=\left(\frac{e}{2 \pi^{2} \epsilon_{0} \gamma r_{b}}\right)^{2} N_{b} \frac{4}{\xi^{2}}\left[\left(\frac{1}{2}-K_{1}\left(\xi_{b}\right) I_{1}\left(\xi_{b}\right)\right)^{2}+\delta\right]
$$

as compared to the $1 \mathrm{D}$ result:

$$
\left\langle\tilde{E}_{k} \tilde{E}_{k}^{*}\right\rangle_{\perp 1 D}=\left(\frac{e}{2 \pi^{2} \epsilon_{0} \gamma r_{b}}\right)^{2} \frac{4 N_{b}}{\xi_{b}^{2}}\left(\frac{1}{2}-K_{1}\left(\xi_{b}\right) I_{1}\left(\xi_{b}\right)\right)^{2} .
$$

We find $\delta\left(\xi_{b}\right)$ is small compared to the dominant terms (as we saw in Fig. 2.15), so we conclude that the 1D and $3 \mathrm{D}$ transverse averages are nearly identical. 


\section{Bibliography}

[1] R. Akre et al. Phys. Rev. ST Accel. Beams, 11:030703, 2008.

[2] L. Wang, Y. Cai, and T. Raubenheimer. Development, characterization and experimental performance of x-ray optics for the lcls free-electron laser. Proceedings of SPIE, 7077, 2008.

[3] http://henke.lbl.gov/optical_constants/.

[4] Z. Huang et al. Measurements of the linac coherent light source laser heater and its impact on the x-ray free-electron laser performance. Phys. Rev. ST Accel. Beams, 13:110703, 2010.

[5] P. Emma et al. First lasing and operation of an ngstrom-wavelength free-electron laser. Nature Photonics, 4:641-647, 2010.

[6] M. Borland. elegant: A flexible SDDS-compliant code for accelerator simulation. Technical Report LS-287, Argonne National Laboratory, 2000.

[7] J.M.J. Madey. Stimulated emission of bremsstrahlung in a periodic magnetic field. J. Appl. Phys., 42:1906, 1971.

[8] Z. Huang and K.J. Kim. Review of x-ray free-electron laser theory. Phys. Rev. ST Accel. Beams, 10:034801, 2007.

[9] L.H. Yu. Generation of intense uv radiation by subharmonically seeded single-pass free-electron lasers. Phys. Rev. A, 44:5178, 1991.

[10] A. Kondratenko and E. Saldin. Generation of coherent radiation by a relativistic electron beam in an ondulator. Particle Accelerators, 10:207-216, 1980.

[11] R. Bonifacio, C. Pellegrini, and L. M. Narducci. Collective instabilities and high-gain regime in a free electron laser. Optics Communications, 50:373-378, 1984.

[12] E. L. Saldin, E. A. Schneidmiller, and M. V. Yurkov. Study of a noise degradation of amplification process in a multistage HGHG FEL. Optics Communications, 202:169, 2002.

[13] Z. Huang. An analysis of shot noise propagation and amplification in harmonic cascade fels. In Proceedings of the 2006 FEL Conference, page 130, Berlin, Germany, 2006.

[14] A. Gover and E. Dyunin. Collective-interaction control and reduction of optical frequency shot noise in charged-particle beams. Phys. Rev. Lett., 102:154801, 2009. 
[15] A. Nause, E. Dyunin, and A. Gover. Optical frequency shot-noise suppression in electron beams. J. of Applied Physics, 107:103101, 2010.

[16] V. Litvinenko. Suppression of shot noise and spontaneous radiation in electron beams. In Proceedings of the 2009 FEL Conference, 2009.

[17] G. Stupakov. Noise amplification in hghg seeding. In Proceedings of the 2010 FEL Conference, Malmo, Sweden, 2010.

[18] E. L. Saldin, E. A. Schneidmiller, and M. V. Yurkov. Nucl. Instrum. Meth. A, 490:1, 2002.

[19] G. Stupakov S. Heifets and S. Krinsky. Phys. Rev. ST-AB, 5:064401, 2002.

[20] Z. Huang and K.J. Kim. Formulas for coherent synchrotron radiation microbunching in a bunch compressor chicane. Phys. Rev. ST Accel. Beams, 5:074401, 2002.

[21] E. Schneidmiller E. Saldin and M. Yurkov. Nucl. Instrum. Meth. A, 528:355, 2004.

[22] Z. Huang, M. Borland, P. Emma, J. Wu, C. Limborg, G. Stupakov, and J. Welch. Suppression of microbunching instability in the linac coherent light source. Phys. Rev. ST-AB, 7:074401, 2004.

[23] T. Shaftan and Z. Huang. Phys. Rev. ST-AB, 7:080702, 2004.

[24] R. Warnock M. Venturini and A. Sholents. Phys. Rev. ST Accel. Beams, 10:054403, 2007.

[25] M. Venturini. Models of longitudinal space-charge impedance for microbunching instability. Phys. Rev. ST Accel. Beams, 11:034401, 2008.

[26] H. Loos et al. In Proceedings of the 2008 Free Electron Laser Conference, 2008.

[27] S. Krinsky M.G. White I. Ben-Zvi, L.F. Di Mauro and L.H. Yu. Proposed uv fel user facility at bnl. Nucl. Instrum. Meth., A304:181-186, 1991.

[28] E. Allaria and G. De Ninno. Extension of the fermi fel1 to shorter wavelengths, mopc002. In Proceedings of the 11th European Particle Accelerator Conference, 2008.

[29] G. Stupakov. Using the beam-echo effect for generation of short-wavelength radiation. Phys. Rev. Lett., 102:074801, 2009.

[30] D. Xiang, E. Colby, M. Dunning, S. Gilevich, C. Hast, K. Jobe, D. McCormick, J. Nelson, T. O. Raubenheimer, K. Soong, G. Stupakov, Z. Szalata, D. Walz, S. Weathersby, M. Woodley, and P.-L. Pernet. Demonstration of the echo-enabled harmonic generation technique for short-wavelength seeded free electron lasers. Phys. Rev. Lett., 105(11):114801, Sep 2010.

[31] T. Shintake. Wavelength compression. Internal Report AccelLab-99-1, KEK, 1999.

[32] T. Shintake. In Proceedings of the 2007 FEL Conference, 2007.

[33] S. Biedron, S. Milton, and H. Freund. Modular approach to achieving the next-generation x-ray light source. Nucl. Instrum. Meth. A, 475:401-406, 2001. 
[34] T. Shaftan and L.H. Yu. High-gain harmonic generation free-electron laser with variable wavelength. Phys. Rev. E, 71:046501, 2005.

[35] D. Ratner, A. Chao, and Z. Huang. Two-chicane compressed harmonic generation of soft x-rays, mopc45. In Proceedings of the 2009 FEL Conference, 2009.

[36] M. Tigner. A possible apparatus for electron clashing-beam experiments. Nuovo Cimento, 37:1228, 1965.

[37] J.M. Klopf et al. The jefferson lab high power thz user facility. Nucl. Instrum. Meth. A, 582:114, 2007.

[38] B.A. Knyazev, G.N. Kulipanov, and N.A. Vinokurov. Novosibirsk terahertz free electron laser: instrumentation development and experimental achievements. Meas. Sci. Technol., 21:054017, 2010.

[39] A. Zholents and W. Fawley. Proposal for intense attosecond radiation from an x-ray free-electron laser. Phys. Rev. Lett., 92:224801, 2004.

[40] A. Zholents, W.M. Fawley, P. Emma, Z. Huang, G. Stupakov, and S. Reiche. In Proceedings of the 2004 FEL Conference, 2004.

[41] G. Geloni, E. Saldin, E. Schneidmiller, and M. Yurkov. Longitudinal impedance and wake from xfel undulators. impact on current-enhanced sase schemes. Nucl. Instrum. Meth. A, 583:228-247, 2007.

[42] H. Chapman, S.P. Hau-Riege, and J. Hajdu. Ultrafast coherent diffraction imaging with x-ray free electron lasers. 2006.

[43] A.A. Varfolomeev, TV. Yarovoi, and P.V. Bousine. Possible enhancement of sase fel output field intensity induced by local phase jump. Nucl. Instrum. and Meth. A, 407:296, 1998.

[44] M. Borland et al. Nucl. Instrum. Meth. A, 483:268, 2002.

[45] S. Heifets and G. Stupakov. Beam instability and microbunching due to coherent synchrotron radiation. Phys. Rev. ST-AB, 5:054402, 2002.

[46] A. Chao D. Ratner and Z. Huang. Three-dimensional analysis of longitudinal space charge microbunching starting from shot noise. In Proceedings of the 2008 Free Electron Laser Conference, 2008.

[47] A. Marinelli and J.B. Rosenzweig. Microscopic kinetic analysis of space-charge induced optical microbunching in a relativistic electron beam. Phys. Rev. ST Accel. Beams, 13:110703, 2010.

[48] K. Bane. Wakefields of sub-picosecond electron bunches, slac-pub-11829. In Workshop on the Physics and Applications of High Brightness Electron Beams, 2005.

[49] M. Chodorow and C. Susskind. Fundamentals of Microwave Electronics. McGraw-Hill, 1964.

[50] J. Rosenzweig, C. Pellegrini, L. Serafinie, C. Ternieden, and G. Travish. The jefferson lab high power thz user facility. Nucl. Instrum. Meth. A, 393:376, 1997.

[51] G. Stupakov and S. Krinsky. Derivation of fel gain using wakefield approach. In Proceedings of the 2003 Particle Accelerator Conference, 2003. 
[52] J.B. Rosenzweig, M.P. Dunning, E. Hemsing, G. Marcus, P. Musumeci, and A. Marinelli. Quasicrystalline beam formation in rf photoinjectors. In Proceedings of the 2008 Free Electron Laser Conference, 2008.

[53] R. Bonifacio, F. Casagrande, and L. DeSalvo Souza. Phys. Rev. A, 33:2836, 1986.

[54] M. Hüning and H. Schlarb. In Proceedings of the 2003 Particle Accelerator Conference, 2003.

[55] P. Emma et al. Commissioning status of the LCLS x-ray fel. In Proceedings of the 2009 Particle Accelerator Conference, 2009.

[56] D. Ratner et al. Fel gain length and taper measurements at lcls, tuoa03. In Proceedings of the 2009 FEL Conference, 2009.

[57] H. Kitamura T. Tanaka and T. Shintake. Nucl. Instrum. Meth. A, 528:172, 2004.

[58] S. Reiche. GENESIS 1.3: a fully 3d time-dependent FEL simulation code. Nucl. Instrum. Meth. A, 429:243-248, 1999.

[59] M. Xie. Design optimization for an x-ray free electron laser drive by slac linac. In Proceedings of PAC95 and IUPAP, pages 183-185, Dallas, TX, 1995.

[60] P.L. Morton N.M. Kroll and M.N. Rosenbluth. Free-electron lasers with variable parameter wigglers. IEEE J. Quant Electron, 17:1436, 1981.

[61] W.M. Fawley, Z. Huang, K.J. Kim, and N.A. Vinokurov. Tapered undulators for sase fels. Nucl. Instrum. Meth. A, 483:537, 2002.

[62] G. Geloni, V. Kocharyan, and E. Saldin. Self-seeded operation of the LCLS hard X-ray FEL in the long-bunch mode. 2010.

[63] R. Bonifacio, L. De Salvo Souza, and P. Pierini. Large harmonic bunching in a high-gain free-electron laser. Nucl. Instrum. Meth. A, 293:627, 1990.

[64] H. Freund, S. Biedron, and S. Milton. Nonlinear harmonic generation in free-electron lasers. IEEE J. Quantum Electron, 36:275, 2000.

[65] Z. Huang and K.J. Kim. Three-dimensional analysis of harmonic generation in high-gain free-electron lasers. Phys. Rev. E, 62:7295, 2000.

[66] A. Tremaine et al. Experimental characterization of nonlinear harmonic radiation from a visible selfamplied spontaneous emission free-electron laser at saturation. Phys. Rev. Lett., 88:204801, 2002.

[67] W. Ackermann et al. Operation of a free-electron laser from the extreme ultraviolet to the water window. Nature Photonics, 1:336-342, 2007.

[68] G. Geloni, E. Saldin, E. Schneidmiller, and M. Yurkov. Paraxial Green's functions in synchrotron radiation theory. Report 05-032, DESY, 2005.

[69] Z. Huang and K.J. Kim. Nonlinear harmonic generation of coherent amplification and self-amplified spontaneous emission. Nucl. Instrum. Meth. A, 475:112-117, 2002. 
[70] S. Biedron et al. Measurements of nonlinear harmonic generation at the advanced photon source's sase fel. Nucl. Instrum. Meth. A, 483:94-100, 2002.

[71] J. Welch et al. X-ray diagnostics commissioning at the lcls. In Proceedings of the 2010 FEL Conference, Malmo, Sweden, 2010.

[72] A. Barty, R. Soufli, T. McCarville, S. Baker, M. Pivovaroff, P. Stefan, and R. Bionta. Predicting the coherent $\mathrm{x}$-ray wavefront focal properties at the linac coherent light source (lcls) $\mathrm{x}$-ray free electron laser. 17:15508, 2009.

[73] H.D. Nuhn et al. Characterization of second harmonic afterburner radiation at the lcls. In Proceedings of the 2010 FEL Conference, Malmo, Sweden, 2010.

[74] E. Saldin, E. Schneidmiller, and M. Yurkov. Properties of the third harmonic of the radiation from self-amplified spontaneous emission free electron laser. Phys. Rev. Lett., 9:030702, 2006.

[75] D. Ratner, A. Chao, and Z. Huang. Two-chicane compressed harmonic generation of soft x-rays. Phys. Rev. ST Accel. Beams, 14:020701, 2011.

[76] J. Qiang. Short wavelength seeding through compression for free electron lasers. Nucl. Instrum. Meth. A, 621:39-46, 2010.

[77] J. Qiang and J. Wu. Generation of coherent x-ray radiation through modulation compression (2010), arXiv:1012.5446v1.

[78] H. Grote and F.C. Iselin. CERN/SL/90-13(AP)(Rev. 5) http://hansg.home.cern.ch/hansg/mad/mad8/mad8.html.

[79] M. Xie. Nucl. Instrum. Meth. A, 445:59, 2000.

[80] Y. Ding et al. Measurements and simulations of ultralow emittance and ultrashort electron beams in the linac coherent light source. Phys. Rev. Lett., 102:254801, 2009.

[81] V.N. Litvinenko, S.F. Mikhailov, O.A. Shevchenko, and Y. Wu. Project for generation of femtosecond x-ray beams from the duke storage ring. In Proceedings of the 2001 Particle Accelerator Conference, page 2614, 2001.

[82] D. Ratner and A. Chao. Steady-state microbunching in a sotrage ring for generating coherent radiation. Phys. Rev. Lett., 105:154801, 2010.

[83] Y. Jiao, D. Ratner, and A. Chao. Coherent THz Radiation from Steady-State Microbunching in storage rings with X-band RF System.

[84] M. Abo-Bakr, J. Feikes, K. Holldack, P. Kuske, W.B. Peatman, U. Schade, G.Wüstefeld, and H.W. Hübers. Brilliant, coherent far-infrared (thz) synchrotron radiation. Phys. Rev. Lett., 90:094801, 2003.

[85] S. Reiche, C. Joshi, C. Pellegrini, J.B. Rosenzweig, S.Ya. Tochitsky, and G. Shvets. Feasibility study of a laser beat-wave seeded thz fel at the neptune laboratory. In Proceedings of the 2005 Particle Accelerator Conference, page 1721, 2005. 
[86] E. Schneidmiller E. Saldin and M. Yurkov. A simple method for the determination of the structure of ultrashort relativistic electron bunches. Nucl. Instrum. Meth. A, 539:499, 2005.

[87] H. Hama et al. Conceptual design of an isochronous ring to generate coherent terahertz synchrotron radiation. New J. Phys., 8:292, 2006.

[88] J.B. Murphy. First observation of simultaneous alpha buckets in a quasi-isochronous storage ring. Phys. Rev. Lett., 84:5516, 2000.

[89] V.K. Neil and A. Sessler. Longitudinal resistive instabilities of intense coasting beams in particle accelerators. Rev.Sci. Instrum., 36:429, 1965.

[90] M. Venturini and R. Warnock. Bursts of coherent synchrotron radiation in electron storage rings: a dynamical model. Phys. Rev. Lett., 89:224802, 2002.

[91] G. Stupakov. Lecture notes on classical mechanics and electromagnetism in accelerator physics, USPAS, June 2007.

[92] J. B. Murphy, S. Krinsky, and R. L. Gluckstern. Longitudinal wakefield for synchrotron radiation. In Proc. IEEE Particle Accelerator Conference and International Conference on High-Energy Accelerators, Dallas, 1995, Piscataway, NJ, 1996. IEEE. (IEEE Conference Record 95CH35843).

[93] Ya. S. Derbenev, J. Rossbach, E. L. Saldin, and V. D. Shiltsev. Microbunch radiative tail-head interaction. DESY FEL Report TESLA-FEL 95-05, Deutsches Elektronen-Synchrotron, Hamburg, Germany, September 1995.

[94] J. Wu, O. Raubenheimer, and G.V. Stupakov. Calculation of the coherent synchrotron radiation impedance from a wiggler. In Proceedings of the 2003 Particle Accelerator Conference, 2003.

[95] E. L. Saldin, E. A. Schneidmiller, and M. V. Yurkov. Radiative interaction of electrons in a bunch moving in an undulator. Nuclear Instruments and Methods, Sec. A, 417:158, 1998.

[96] B.W.J. McNeil, G.R.M. Robb, M.W. Poole, and N.R. Thompson. Harmonic lasing in a free-electronlaser amplifier. Phys. Rev. Lett., 96:084801, 2006.

[97] D. Ratner, A. Chao, and Z. Huang. Enhancing fel power with phase shifters, mopph023. 2007.

[98] Linac Coherent Light Source (LCLS) conceptual design report. Report SLAC-R-593, SLAC, 2002.

[99] Tesla technical design report. Report TESLA FEL 2002-09, DESY, 2002. 